\title{
Feasibility Study of Pressure Pulsing Pipeline Unplugging Technologies for Hanford
}

\author{
Author Name: \\ M.A. Servin \\ Washington River Protection Solutions, LLC \\ Richland, WA 99352 \\ U.S. Department of Energy Contract DE-AC27-08RV14800

$\begin{array}{llll}\text { EDT/ECN: } & \text { DRF } & \text { UC: N/A } \\ \text { Cost Center: } & & \text { Charge Code: } & \text { N/A } \\ \text { B\&R Code: } & \text { N/A } & \text { Total Pages: } 210\end{array}$

Key Words: Pipeline, Unplugging, Pulsing, Pressure, Pipe-Pulse, Hydrokinetics, Feasibility, Transfer Line, Recovery, Cross Site, Plugging, Pressure Pulse, Vibration, Plug, Waste Transfer System

Abstract: The ability to unplug key waste transfer routes is generally essential for successful tank farms operations. All transfer lines run the risk of plugging but the cross site transfer line poses increased risk due to its longer length. The loss of a transfer route needed to support the waste feed delivery mission impacts the cost and schedule of the Hanford clean up mission. This report addresses the enfineering feasibility for two pressure pulse technologies, which are similar in concept, for pipeline unplugging.

TRADEMARK DISCLAIMER. Reference herein to any specific commercial product, process, or service by trade name, trademark, manufacturer, or otherwise, does not necessarily constitute or imply its endorsement, recommendation, or favoring by the United States Government or any agency thereof or its contractors or subcontractors.

\section{APPROVED}

By G. E. Bratton at 10:58 am, Jan 02, 2013

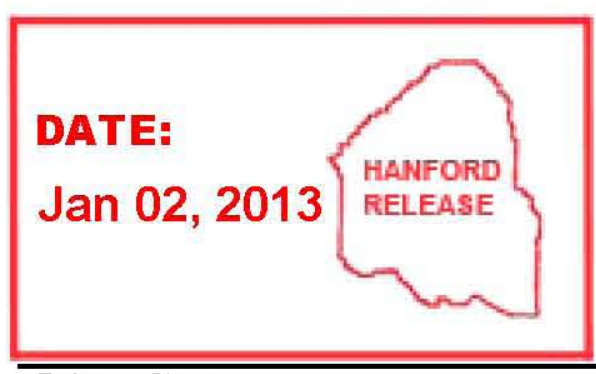

Release Stamp

\section{Approved For Public Release}


RPP-RPT-53783

Rev. 0

\section{Feasibility Study of Pressure Pulsing Pipeline Unplugging Technologies for Hanford}

J. S. Garfield

G. R. Golcar

A E M Consulting, LLC

M. A. Servin

Washington River Protection Solutions, LLC

Date Published

December 18, 2012

Prepared for the U.S. Department of Energy Office of River Protection

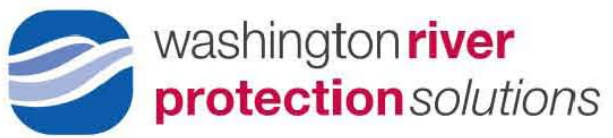

P.O. Box 850

Richland, Washington 


\section{EXECUTIVE SUMMARY}

Washington River Protection Solutions, LLC (WRPS) recently conducted a preliminary literature survey of commercial technologies suitable for use in pipeline unplugging in the Hanford Site waste transfer system. ${ }^{1}$ The survey resulted in the down-selection of two pressure pulse technologies, which are similar in concept, for further evaluation. This report addresses the engineering feasibility for the two selected options: Hydrokinetics ${ }^{2}$ technology by AIMM Technologies, Inc., and Pipe-Pulse technology by Paradigm Flow Services, Ltd.

An overview of the Pipe-Pulse technology is provided. A strict nondisclosure agreement required by Paradigm Flow Services prevented detailed discussion with staff regarding operation and design details of the Pipe-Pulse technology. The limited evaluation of the Pipe-Pulse technology focused on the Pipe-Pulse patent application filing and public domain literature. The Hydrokinetics system has been used to successfully unplug pipes with pressure pulses of 2-30 cycles per minute since 1996. The pulses from a high-pressure piston pump ( $20 \mathrm{gal} / \mathrm{min})$ create a standing wave in the nearly incompressible fluids that frees a plug from adherence to the pipe wall. The system shock (0-20 $\mathrm{gal} / \mathrm{min})$ is well below the stress caused by a water hammer incident.

The theory of operation is that the standing wave introduced into a plugged line causes the plug and pipe wall to resonate at different frequencies to break the bond between the waste plug and pipe wall. The plug is moved by a combination of positive head pressure and pulse action.

Localized acoustic cavitation at the interface between the plug and pipe wall also serves to break the plug free.

At Hanford, the maximum pressure allowed may vary, depending on the age and design pressure for each pipe route and the weakest jumper components in the route. WRPS is currently conducting water hammer stress analyses for the double-shell tank pipe routes. A similar stress analysis can be used to determine maximum pressures when using a pressure pulse process for pipeline unplugging.

The Hydrokinetics equipment can be deployed in permanent facilities as a modification to current flushing system upgrades in the double-shell tank farm valve pits. Many of the flushing and unplugging functions overlap, and these operations could be combined. The Hydrokinetics technology can also be deployed at any valve pit as a portable trailer-mounted system. The portable system functions are similar to the current hydrostatic pipeline pressure testing used in the tank farms.

The ability to unplug key waste transfer routes is generally essential for successful tank farms operations and specifically needed for the anticipated Tank AY-102 transfers and cross-site transfers. The loss of a transfer route needed for waste feed delivery to the Waste Treatment and Immobilization Plant impacts the cost and schedule of the Hanford cleanup mission. The advantages of using a pressure pulse technology like Hydrokinetics warrant additional testing and the development of a design approach suited for Hanford tank farms application.

The technology has demonstrated success in private industry, and the costs of implementing a pressure pulse system are reasonable.

\footnotetext{
${ }^{1}$ RPP-RPT-52347, 2012, Pipeline Unplugging Assessment and Recommendations for Hanford Waste Transfer Systems, Rev. 0, Washington River Protection Solutions, LLC, Richland, Washington.

${ }^{2}$ Hydrokinetics is a trademark of AIMM Technologies, Inc., Texas City, Texas.
} 


\section{CONTENTS}

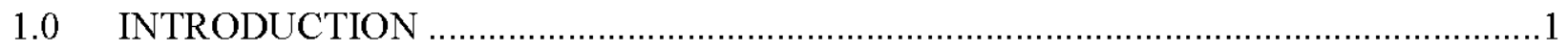

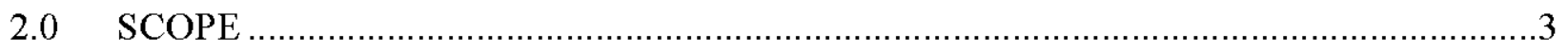

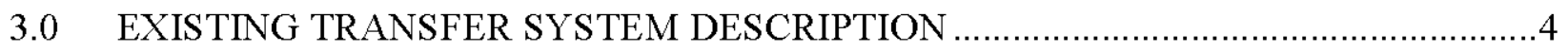

3.1 Component Description............................................................................

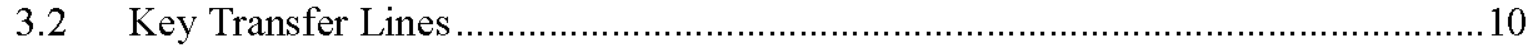

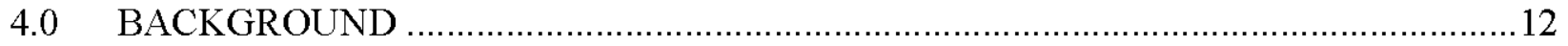

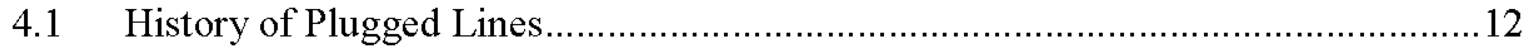

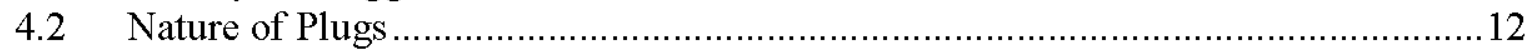

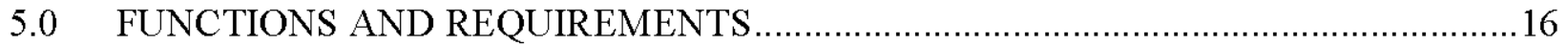

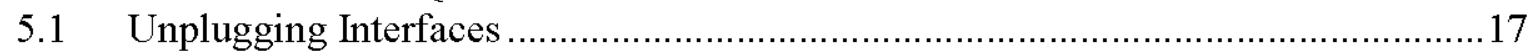

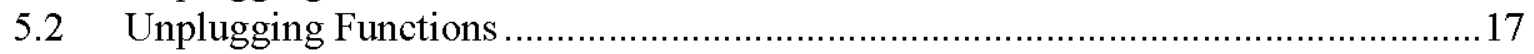

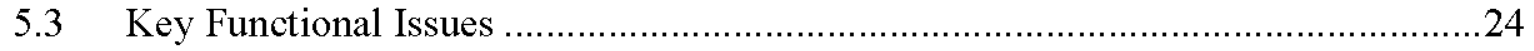

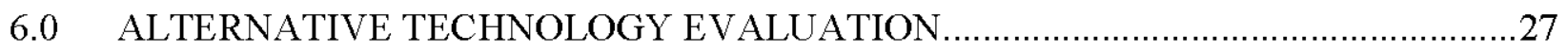

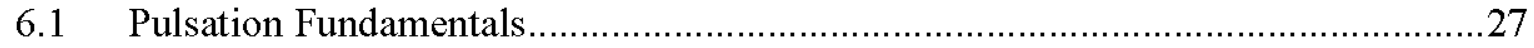

6.2 Transport and Blockage Removal Approach ……….........................................30

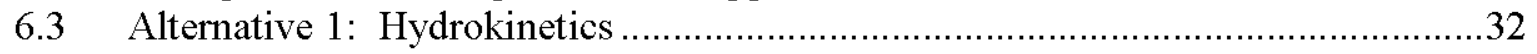

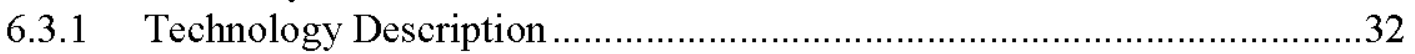

6.3.2 Existing Field Deployment …………………….................................

6.3.3 Scoping Tests with Pipeline Plug Simulants.............................................. 37

6.3.4 Deployment Safety Aspects ...............................................................41

6.3.5 Regulatory Impacts ...................................................................... 43

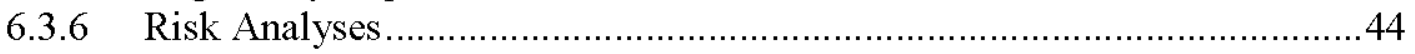

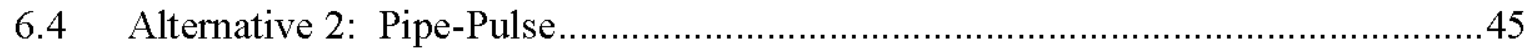

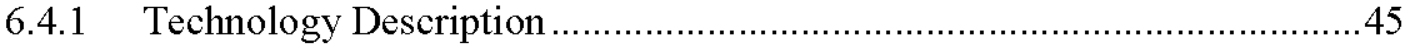

6.4.2 Deployment Safety Aspects, Regulatory Impacts, and Risks Analysis ......48

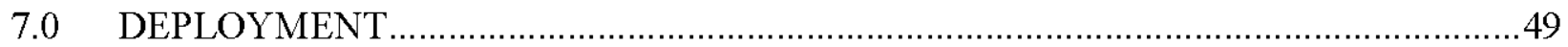

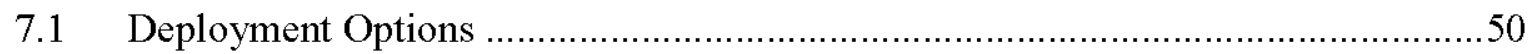

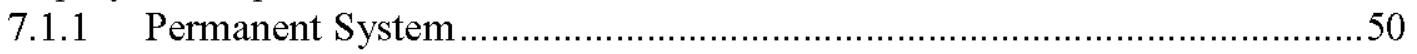

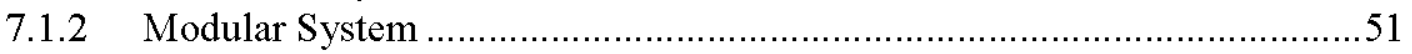

7.1.3 Configuration Summary ……………………................................5

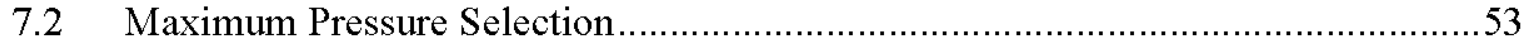

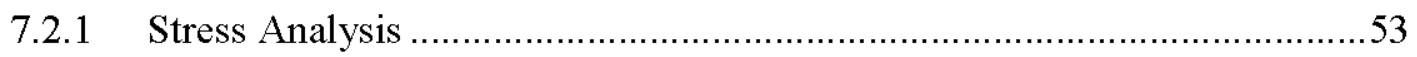

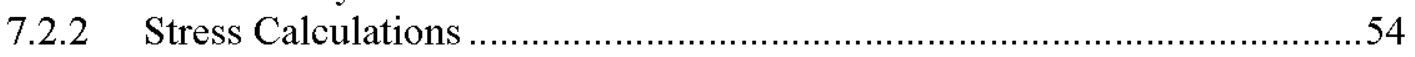

7.2.3 Summary of Pressure Discussion...........................................................55

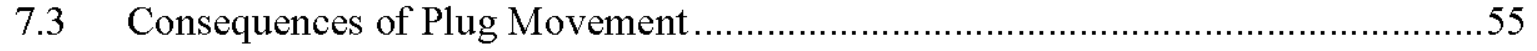

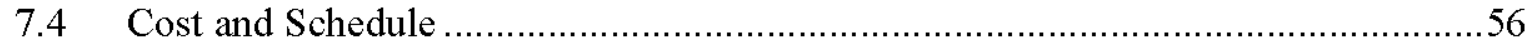

7.4.1 Permanent Facility Costs .......................................................................56

7.4.2 Portable Facility Costs ...........................................................................

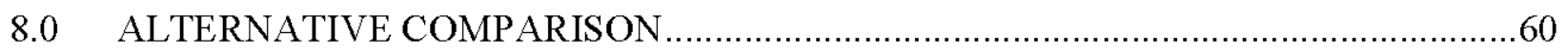

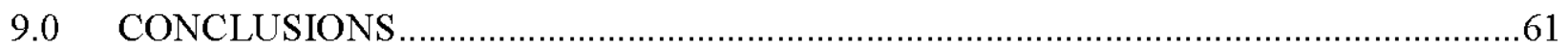

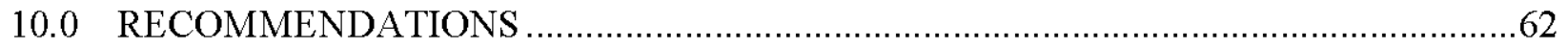

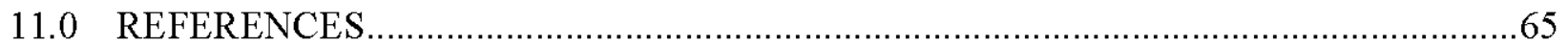




\section{APPENDICES}

Appendix A Hydrokinetics Patent ...................................................................... A

Appendix B AIMM Technologies' Hydrokinetic Process Technology Assessment .............. B-i

Appendix C AIMM Article, "A Better Way to Clean".................................................. C-i

Appendix D Pipeline Unplugging Assessment and Recommendations for the Fernald

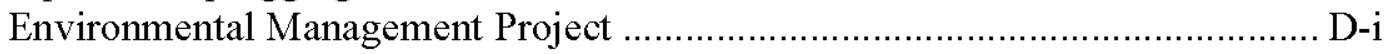

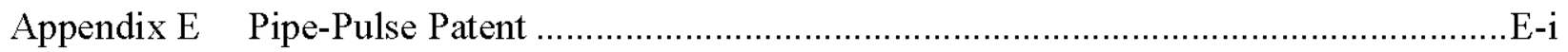

Appendix F Diluent and Flush Building Costs .......................................................... F-i

Appendix G Hydrokinetics Correspondence ..................................................................... G-i 


\section{FIGURES}

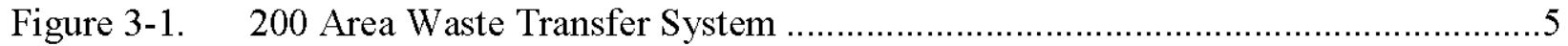

Figure 3-2. Details of Expansion Joints for Cross-Site Line …….......................................

Figure 3-3. Expansion Joint Layout for Cross-Site Lines ( $\sim 600-\mathrm{ft}$ intervals) ..........................

Figure 3-4. Example of Pipeline Expansion Joints (100-ft intervals) ………........................ 10

Figure 4-1. Photomicrograph of $\mathrm{Na}_{3} \mathrm{PO}_{4} \bullet 12 \mathrm{H}_{2} \mathrm{O}$ Crystals at $63 \times$ Magnification...................14

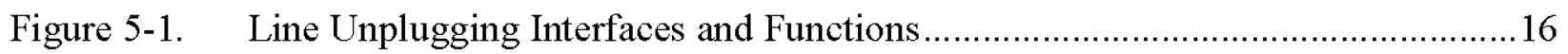

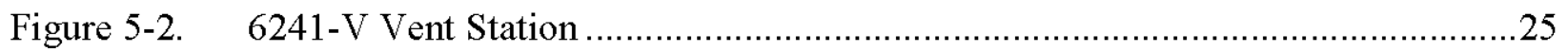

Figure 6-1. Transfer Operation and Pulsation Unplugging Logic.........................................30

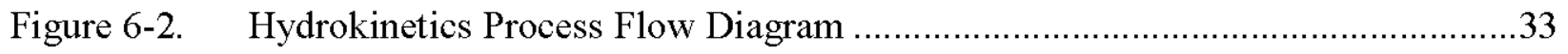

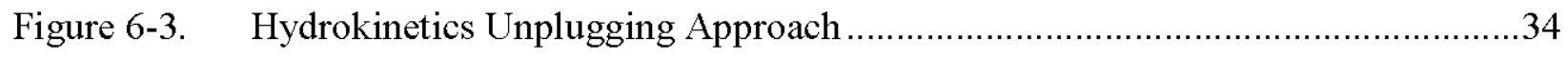

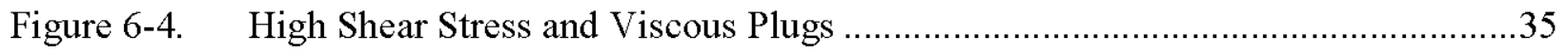

Figure 6-5. Pipeline Blockage or Foulants Handled by the Hydrokinetics System .................36

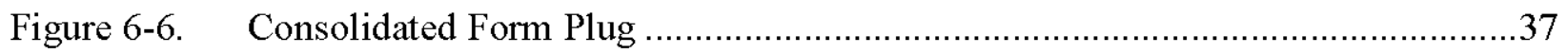

Figure 6-7. Illustrations of Test Pipeline Length Configurations........................................38

Figure 6-8. Pipe-Pulse System Process and Instrumentation .............................................4

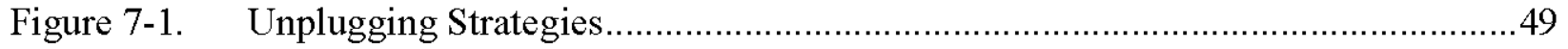

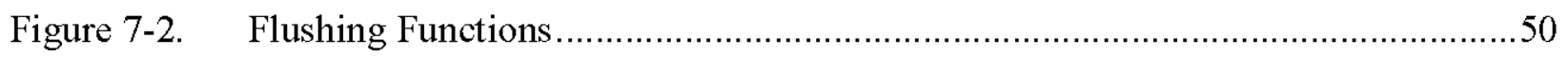

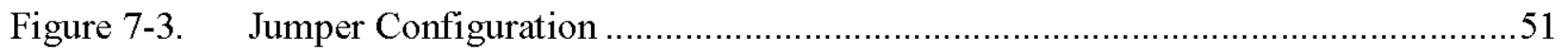

Figure 7-4. Hydrokinetics Modified Process Diagram......................................................52

Figure 7-5. AY/AZ Farm Diluent and Flush System Piping and Instrumentation

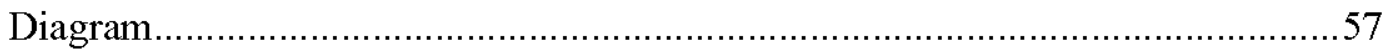

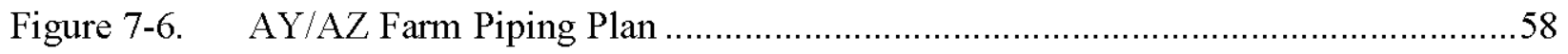

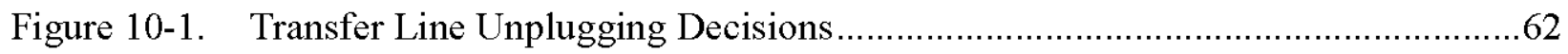

\section{TABLES}

Table 3-1. Design Temperatures and Pressures for Transfer Primary Pipe ……....................

Table 4-1. Hanford Pipeline Plugging Literature Survey Summary.....................................13

Table 5-1. Unplugging Functional Requirements (3 pages) …….....................................21

Table 6-1. Water Hammer Pressure Surge. ......................................................................

Table 6-2. Hydrokinetics System Unplugging Tests at Florida International

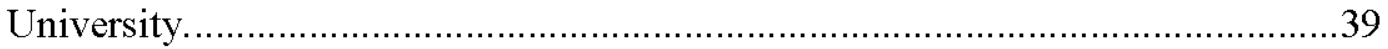

Table 7-1. Comparison of Permanent vs. Modular Unplugging Systems .............................51

Table 7-2. AY/AZ Farm Diluent and Flush Facility Project T3W 12 ..................................56 


\section{TERMS}

\section{Acronyms and Abbreviations}

\begin{tabular}{|c|c|}
\hline ALARA & as low as reasonably achievable \\
\hline ASME & American Society of Mechanical Engineers \\
\hline ASTM & American Society for Testing and Materials \\
\hline DOE & U.S. Department of Energy \\
\hline DSA & documented safety analysis \\
\hline DST & double-shell tank \\
\hline Ecology & Washington State Department of Ecology \\
\hline EIS & environmental impact statement \\
\hline ETF & Effluent Treatment Facility \\
\hline EPA & U.S. Environmental Protection Agency \\
\hline FIU & Florida International University \\
\hline FIV & flow-induced vibration \\
\hline HLW & high-level waste \\
\hline IDF & Integrated Disposal Facility \\
\hline IQRPE & Independent Qualified Registered Professional Engineer \\
\hline LAW & low-activity waste \\
\hline LERF & Liquid Effluent Retention Facility \\
\hline MARS & mobile arm retrieval system \\
\hline MTTR & mean time to restore \\
\hline NEPA & National Environmental Policy Act \\
\hline OR & operations research \\
\hline ORNL & Oak Ridge National Laboratory \\
\hline P\&ID & piping and instrumentation diagram \\
\hline PLC & programmable logic controller \\
\hline PUREX & plutonium uranium extraction \\
\hline RCRA & Resource Conservation and Recovery Act \\
\hline $\mathrm{RCW}$ & Revised Code of Washington \\
\hline REDOX & reduction-oxidation \\
\hline RTRP & reinforced thermosetting resin pipe \\
\hline SST & single-shell tank \\
\hline TC \& WM & Tank Closure and Waste Management \\
\hline WAC & Washington Administrative Code \\
\hline WFD & waste feed delivery \\
\hline WO & World Intellectual Property Organization \\
\hline WRF & waste retrieval facility \\
\hline WRPS & Washington River Protection Solutions, LLC \\
\hline WTP & Waste Treatment and Immobilization Plant \\
\hline
\end{tabular}




$\begin{array}{ll}\text { Units } & \\ { }^{\circ} \mathrm{C} & \text { degrees Centigrade } \\ { }^{\circ} \mathrm{F} & \text { degrees Fahrenheit } \\ \mu & \text { micron } \\ \mathrm{ft} & \text { feet } \\ \mathrm{ft} / \mathrm{sec} & \text { feet per second } \\ \mathrm{g} / \mathrm{cm}^{3} & \text { grams per cubic centimeter } \\ \mathrm{gal} & \text { gallon } \\ \mathrm{gal} / \mathrm{min} & \text { gallons per minute } \\ \mathrm{Hz} & \text { hertz } \\ \mathrm{in} . & \text { inch } \\ \mathrm{K} & \text { thousands } \\ \mathrm{lb} & \text { pound } \\ \mathrm{lbf} / \mathrm{in}^{2} & \text { pound force per square inch } \\ \mathrm{Lft} & \text { linear feet } \\ \mathrm{m} & \text { molal } \\ \mathrm{M} & \text { molar } \\ \mathrm{Mgal} & \text { million gallons } \\ \mathrm{mi} & \text { mile } \\ \mathrm{MPa} & \text { megapascal } \\ \mathrm{psi} & \text { pounds per square inch } \\ \mathrm{psig} & \text { pounds per square inch, gauge } \\ \mathrm{VAC} & \text { volts alternating current } \\ \mathrm{wt} \% & \text { weight percent } \\ & \end{array}$




\section{TRADEMARK DISCLOSURE}

Bondstrand is a registered trademark of NOV Fiber Glass Systems, San Antonio, Texas.

Fibercast is a registered trademark of Varco I/P, Inc., Houston, Texas.

Hydrokinetics is a trademark of AIMM Technologies, Inc., Texas City, Texas.

Victaulic is a registered trademark of Victaulic Company, Easton, Pennsylvania. 


\subsection{INTRODUCTION}

The Hanford Site waste tank system consists of buried and encased (pipe-in-pipe) 2-in. and 3-in. lines used for transfer of high-level waste (HLW) slurries containing undissolved solids and 3-in. lines for supernatant wastes containing dissolved salts. The transfer lines are used to move wastes between tanks and for waste feed delivery to the Waste Treatment and Immobilization Plant (WTP). Waste transfer line plugging has occurred due to the following:

- Settled undissolved solids

- Waste chemistry instability caused by cooling supernatant and solutes reaching saturation point

- Hydrodynamic instability and transition from turbulent to laminar

- Concentrations of phosphate-forming gels.

Procedural precautions taken to assess waste chemistry before transfers to avoid line plugging include managing the waste concentrations and the transfer rate to keep solids in solution or in suspension during the transfer (TFC-ENG-STD-26, Waste Transfer, Dilution, and Flushing Requirements). However, the risk and consequences associated with a plugged transfer line warrant having a viable strategy for recovery.

A 2012 transfer line reliability report (RPP-RPT-51921, The Phase 3.1 Hanford Waste Feed Delivery Operations Research Model Bases and Assumptions) indicates that due to the line lengths, the cross-site transfer lines have a higher risk of failure and a higher recovery consequence for replacement in terms of cost and schedule. The cross-site transfer lines are essential for transfers from the 200 West Area to 200 East Area and if not operating when needed, will impact the overall WTP processing mission.

Line plugging is identified as a formal risk in TFC-PLN-39, Risk and Opportunity Management Plan, as TOC-12-012, "WFD/Transfer Line Plugging." In addition, RPP-46506, Decision Framework Key Decisions Required to Achieve the RPP Mission, lists Decision 2.3 as, "Determine what enhancements and improved configurations are needed for the cross-site slurry transfer system," and includes "Development of plug removal technologies."

The U.S. Department of Energy (DOE) has funded unplugging tests at Florida International University (FIU) since 2000. The reviewed technologies have focused on a pulse technology relevant to this evaluation.

Washington River Protection Solutions, LLC (WRPS) recently conducted a preliminary literature survey of commercial technologies suitable for use in pipeline unplugging in the Hanford Site waste transfer system (RPP-RPT-52347, Pipeline Unplugging Assessment and Recommendations for Hanford Waste Transfer Systems). The survey resulted in the downselection of two pressure pulse technologies for further evaluation. This report addresses the two selected options: Hydrokinetics ${ }^{3}$ technology by AIMM Technologies, Inc., and Pipe-Pulse technology by Paradigm Flow Services, Ltd.

\footnotetext{
${ }^{3}$ Hydrokinetics is a trademark of AIMM Technologies, Inc., Texas City, Texas.
} 
Both vendors offer an unplugging approach that applies pressure in the plugged line and pulses the pressure at various frequencies and amplitude. Both have demonstrated experience unplugging lines in the chemical and petroleum industries.

Hydrokinetics has been in operation since 1990. Paradigm Flow Services is newly founded (2010), and the Pipe-Pulse technology has completed proof-of-principle demonstration in a pilot umbilical flow line (Chesshyre 2011). This study evaluates the vendor patents, literature, experience, and equipment, and identifies a means of deployment applicable to the Hanford Site line plugging issue.

The following appendices provide supplemental information to this report:

- Appendix A, Hydrokinetics Patent

- Appendix B, AIMM Technologies' Hydrokinetic Process Technology Assessment

- Appendix C, AIMM Article, "A Better Way to Clean"

- Appendix D, Pipeline Unplugging Assessment and Recommendations for the Fernald Environmental Management Project

- Appendix E, Pipe-Pulse Patent

- Appendix F, Diluent and Flush Building Costs

- Appendix G, Hydrokinetics Correspondence. 


\subsection{SCOPE}

Due to the similar nature of the Hydrokinetics and Pipe-Pulse systems, this evaluation focuses on a means for implementing a suitable pressure pulse technology for unplugging lines in the Hanford Site double-shell tank (DST) waste transfer system. The following areas were addressed in the course of this study.

- Waste Transfer System Overview - A brief description is provided of the Hanford waste transfer system, including component descriptions and key transfer lines.

- Line plugging background - The history of line plugging and the chemistry involved in line plugging were reviewed.

- FIU testing - The FIU test reports were reviewed and participants were interviewed to better understand unplugging pressure requirements and the associated pulse frequency and amplitude.

- Vendor experience - Vendor literature was reviewed and the operating and engineering personnel of the Hydrokinetics technology were interviewed for theoretical, practical, and empirical experience with pipeline unplugging. A strict nondisclosure agreement required by Paradigm Flow Services for engagement in technical discussions on the PipePulse technology limited the evaluation for that alternative.

- Functional analysis - The functional requirements for implementing a pressure pulse technology were compared to the current approach used for pipeline flushing and hydrostatic pressure testing.

- Implementation approach - The option to incorporate a permanent pressure pulsation unplugging capability into the current flushing system was compared to a portable trailermounted system deployed like a hydrostatic test module.

- Stress analysis - Fitness-for-service requirements and a recently completed water hammer stress analysis were reviewed for findings relevant to the pressure pulse technology.

- Operability - The design approach, line pressures, and sequence needed for successful implementation were reviewed with tank farms engineering and operating personnel for compliance with the existing design parameters and operating philosophy.

- Cost and schedule - The pressure pulse equipment and system operations were scoped at a preconceptual level to approximate the cost and schedule associated with procurement, integration with existing facilities/utilities, and setup.

- Safety and regulatory - Safety and regulatory risks and mitigating actions were identified.

- Alternatives comparison - The pressure pulse technology alternatives were compared with regard to pressure, frequency amplitude, and field implementation in the tank farms valve pits; a basis for selection is presented along with options for future work.

- Path forward - Recommendations are made to facilitate further development of the pressure pulse technology for use in the Hanford waste transfer system. 


\subsection{EXISTING TRANSFER SYSTEM DESCRIPTION}

The Hanford Site stores approximately $56 \mathrm{Mgal}$ of Cold War-era radioactive and chemically hazardous waste in 177 underground tanks. Of the 177 tanks, 28 are DSTs and 149 are singleshell tanks (SST). The waste will be vitrified in new treatment facilities currently under construction. About 10 percent of the tank waste will be treated as HLW, which will be disposed of in a deep geological repository that is yet to be determined. Approximately 90 percent of the tank waste will be treated as low-activity waste (LAW) that will be disposed at the Hanford Site Integrated Disposal Facility (IDF). Waste is being retrieved from the SSTs and queued in the DSTs for processing. Figure 3-1 depicts the 200 Area waste transfer system.

The DSTs are organized into six tank farms, with underground waste transfer lines connecting the tank farms. Within each tank farm is another set of transfer lines that can be used to move waste between tanks within the farm or to connect with the inter-farm transfer lines. Each tank has one or two transfer lines that originate in a below-grade concrete structure called a pump pit and end in a similar structure called a valve pit. At least one valve pit is centrally located in each tank farm. Inside the valve and pump pits, the transfer lines and equipment are connected by short sections of pipe, called jumpers, that provide flexibility and remote-maintenance capability for the transfer line network. The jumpers, which may contain one or more valves each, can be connected so that waste can be routed between any two tanks in the waste transfer system.

Some of the shorter, temporary routes are made with hose-in-hose aboveground transfer lines. SST waste retrieval transfers are made through hose-in-hose transfer lines. If a plug occurs in the hose-in-hose transfer lines, the aboveground hoses are replaced on these routes.

The Hanford waste transfer system consists of buried and encased (pipe-in-pipe) 2-in. and 3-in. lines used for transfers of HLW slurries containing undissolved solids and 3-in. lines for supernatant wastes containing dissolved salts. The transfer lines are used to move wastes between tanks and for waste feed delivery to the WTP. The lines originate and terminate in valve pits with a special plutonium-uranium extraction (PUREX) connector nozzle. All routes are sloped a minimum of 3 in. per $100 \mathrm{ft}$ to drain free liquids. Routes can be changed in the valve pits with valve settings or by removing the cover blocks with a crane and remotely loosening the PUREX connectors and changing the jumpers.

The transfer routes typically consist of several lines and jumper combinations. Transfer lines between tanks are typically several hundred feet long. Transfer lines to the WTP are 3,000-5,000 $\mathrm{ft}$ long, and the cross-site lines connecting the SY Farm in the $200 \mathrm{West}$ Area with the AN Farm in 200 East Area are approximately $5 \mathrm{mi}$ or $29,000 \mathrm{ft}$ in length. Many of the older lines are rated at $275 \mathrm{psig}$ design pressure, while the newer lines are rated at $400 \mathrm{psig}, 1,000 \mathrm{psig}$, or 1,490 psig. The burst pressure for Type 304L stainless pipe is over 9,000 psig (ASTM A312, Standard Specification for Seamless, Welded, and Heavily Cold Worked Austenitic Stainless Steel Pipes). Maximum pressures are limited by the weakest portions of any route, which are often the PUREX connectors (400 psig). The pressure limitations for unplugging the transfer lines are therefore 275 or 400 psig, unless the weak links are not part of the unplugging effort.

The following subsections describe major components and key transfer lines of the Hanford waste transfer system. 


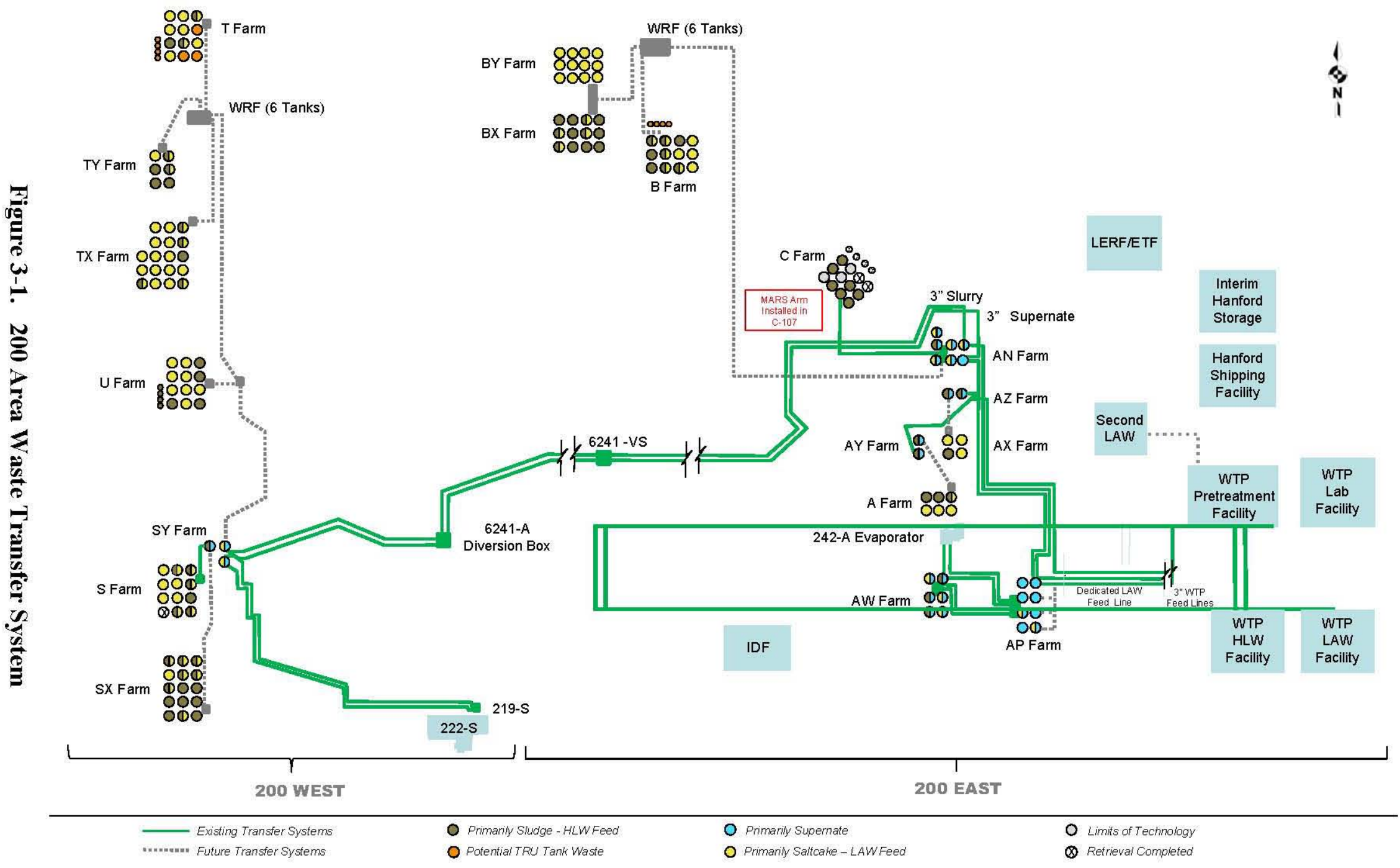




\subsection{COMPONENT DESCRIPTION}

Valve Pits - There are eight interconnected valve pits in the six DST farms. Each of the 28 DSTs have a pump pit with a similar "pit" concept. The valve pits have concrete cover blocks for shielding and include jumpers with valves to configure the desired transfer route. There are approximately 220 DST and SST jumpers in the waste transfer system. In the pits, jumpers are connected to the wall nozzles with PUREX connectors. Jumper lengths vary from 3-20 Lft of pipe. Waste transfer system jumpers include 2 -in. Schedule 40 pipe for slurry transfers and 3-in. Schedule 40 pipe for supernatant transfers. In many cases, jumpers contain at least one valve to facilitate the redirection or isolation of flow through the jumper to the desired waste transfer line.

There are approximately 215 waste transfer valves and actuators in the waste transfer system. The valves are typically 2 -in. and 3 -in. full port, 1/4-turn ball 2 -way valves, and 2 -in. and 3 -in. T-ported 3-way ball valves. The valves are manually operated with T-handles that extend through the pit cover block to allow shielding for remote operation.

Unplugging waste transfer lines through the jumpers in the valve pits must address the maximum pressure on the PUREX connector and on any valves in the jumper system. The seals in the PUREX connectors, valve stems, and valve flanges are the weak points in the system. The valves and PUREX connectors have 90-degree elbows that can collect solids and restrict the unplugging potential.

Transfer Lines - There are currently 75 active transfer lines (typically 3 -in. in 6-in. encasement Schedule 40 supernatant/slurry lines, and 2-in. in 4-in. encasement Schedule 40 slurry lines) ranging in age from 1 to 35 years. The primary pipes are either A53 Type S, Grade B carbon steel (ASTM A53, Standard Specification for Pipe, Steel, Black and Hot-Dipped, Zinc-Coated, Welded and Seamless) or, since 1986, ASTM A312 Type 304L stainless steel. The 219-S transfer lines and encasements are made of Bondstrand/Fibercast ${ }^{4}$ filament-wound reinforced thermosetting resin pipe (RTRP), with a resin corrosion barrier. These lines consist of a new section of piping that ties into an old existing section of piping. The newer piping conforms to ASTM D2996, Standard Specification for Filament-Wound "Fiberglass" (Glass-FiberReinforced Thermosetting-Resin) Pipe, Type I, Grade 1, Class F. The older piping conforms to ASTM D2997, Standard Specification for Centrifugally Cast "Fiberglass" (Glass-Fiber-Reinforced Thermosetting-Resin) Pipe, Type II, Grade 1, Class C. The encasements are either ASTM A53, Type S, Grade B, or ASTM A106, Standard Specification for Seamless Carbon Steel Pipe for High-Temperature Service, Grade B with various forms of special protective coatings.

Standard expansion joints are built into the waste transfer lines to allow expansion and contraction by thermocycling from ambient to $200^{\circ} \mathrm{F}$ and up to $340^{\circ} \mathrm{F}$. Typically, the lines are offset $10 \mathrm{ft}$ for every $100 \mathrm{ft}$ in length, with two long radius 90 -degree elbows. The cross-site transfer lines have U-shaped expansion joints every $600 \mathrm{ft}$ that are offset $25 \mathrm{ft}$ by $12 \mathrm{ft}$ with 36-in. bend radius elbows.

\footnotetext{
${ }^{4}$ Bondstrand is a registered trademark of NOV Fiber Glass Systems, San Antonio, Texas, and Fibercast is a registered trademark of Varco I/P, Inc., Houston, Texas.
} 
The waste transfer lines are buried at nominal depths of $2.5 \mathrm{ft}$ to $4 \mathrm{ft}$, with minimum depths as shallow as $1.65 \mathrm{ft}$ (RPP-18652, Buried Pipe Analysis for DST System Integrity Assessment). The lines are sloped a minimum of $3 \mathrm{in}$. in $100 \mathrm{ft}(0.25$ percent $)$ to facilitate complete draining of liquids and to minimize the corrosion impacts over the life of the pipe. A summary of DST transfer line properties is provided in Table 3-1. The design pressures and temperatures vary depending on use. Slurry transfers require additional pump pressure to accomplish the transfer at a minimum velocity of $6 \mathrm{ft} / \mathrm{sec}$ to prevent solids settling. The cross-site lines and WTP feed route are longer and require additional pump pressure to travel the entire distance.

Table 3-1. Design Temperatures and Pressures for Transfer Primary Pipe

\begin{tabular}{|c|c|c|c|}
\hline \multirow[b]{2}{*}{ Tank farm/key routes } & \multicolumn{2}{|c|}{ Design pressure $\left(\mathrm{lb} / \mathrm{in}^{2} \mathrm{~g}\right)$} & \multirow{2}{*}{$\begin{array}{l}\text { Design temperature } \\
\qquad\left({ }^{\circ} \mathrm{F}\right)\end{array}$} \\
\hline & SN & SL & \\
\hline 241-AN & 275 & 400 & 340 \\
\hline 241-AP & 400 & 400 & 340 \\
\hline 241-AW & 275 & 400 & 340 \\
\hline 241-AY & 400 & 400 & 200 \\
\hline 241-AZ & 400 & 400 & 200 \\
\hline 241-SY (pipe) & 230 & 400 & 330 \\
\hline 241-SY (hose) & 425 & 425 & 180 \\
\hline Cross-Site SNL-3150, SLL-3160 & 1,490 & 1,490 & 200 \\
\hline WTP feed HLW SN-637, LAW SN-700, SN-701 & 1,000 & 1,000 & 200 \\
\hline
\end{tabular}

Source: RPP-15137, 2011, System Design Description for the 200 East Area DST Waste Transfer System (DSA-Based), Rev. 6, Washington River Protection Solutions, LLC, Richland, Washington.

Note: Complete design specifications for each specific line are listed in Table B.1 of RPP-RPT52206, 2012, Tank Farms Waste Transfer System Fitness-for-Service Requirements and Recommendations, Rev. 0, Washington River Protection Solutions, LLC, Richland, Washington.

$\mathrm{HLW}=$ high-level waste. $\quad$ WTP $\quad=$ Waste Treatment and Immobilization Plant.

LAW $\quad=$ low-activity waste.

Characteristic layouts are shown in Figure 3-2 through Figure 3-4. Figure 3-2 shows the typical expansion joints and bends in the cross-site transfer lines. Figure 3-3 shows the expansion joints in a plan view for a portion of the line. Figure 3-4 shows the 10-ft offset at approximately 100-ft intervals for the remaining DST transfer lines. 

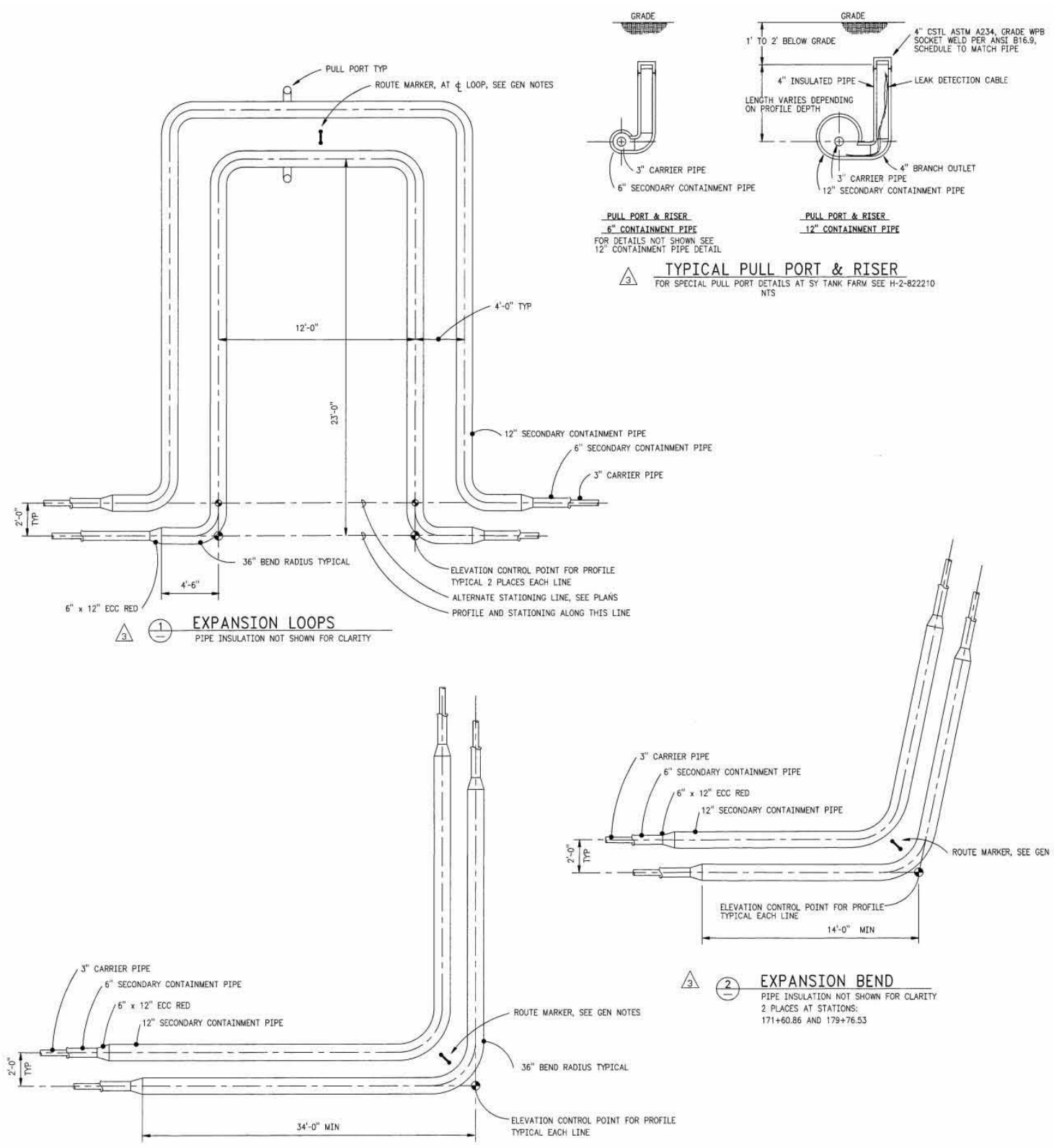

(3) 3) $90^{\circ}$ EXPANSION CORNER

2P PLACES AT STATITONS:
$194+11.04$ ANO $198+70.04$

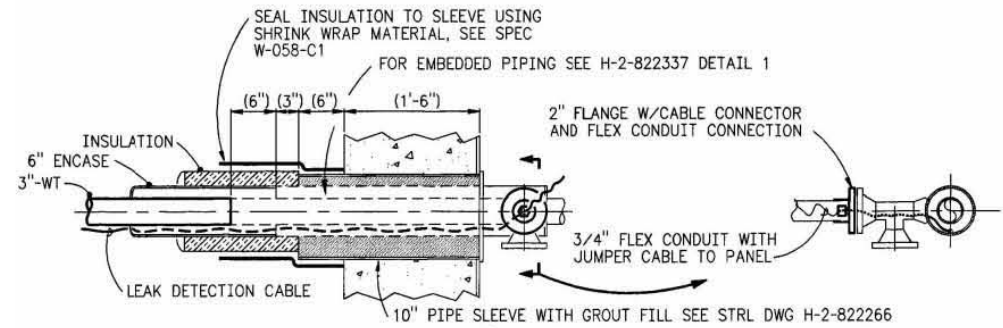

TYPICAL TRANSFER LINE TIE-IN

Source: H-2-822209, 1995, "Civil General Notes and Details," Sheet 1, Rev. 3.

Figure 3-2. Details of Expansion Joints for Cross-Site Line 


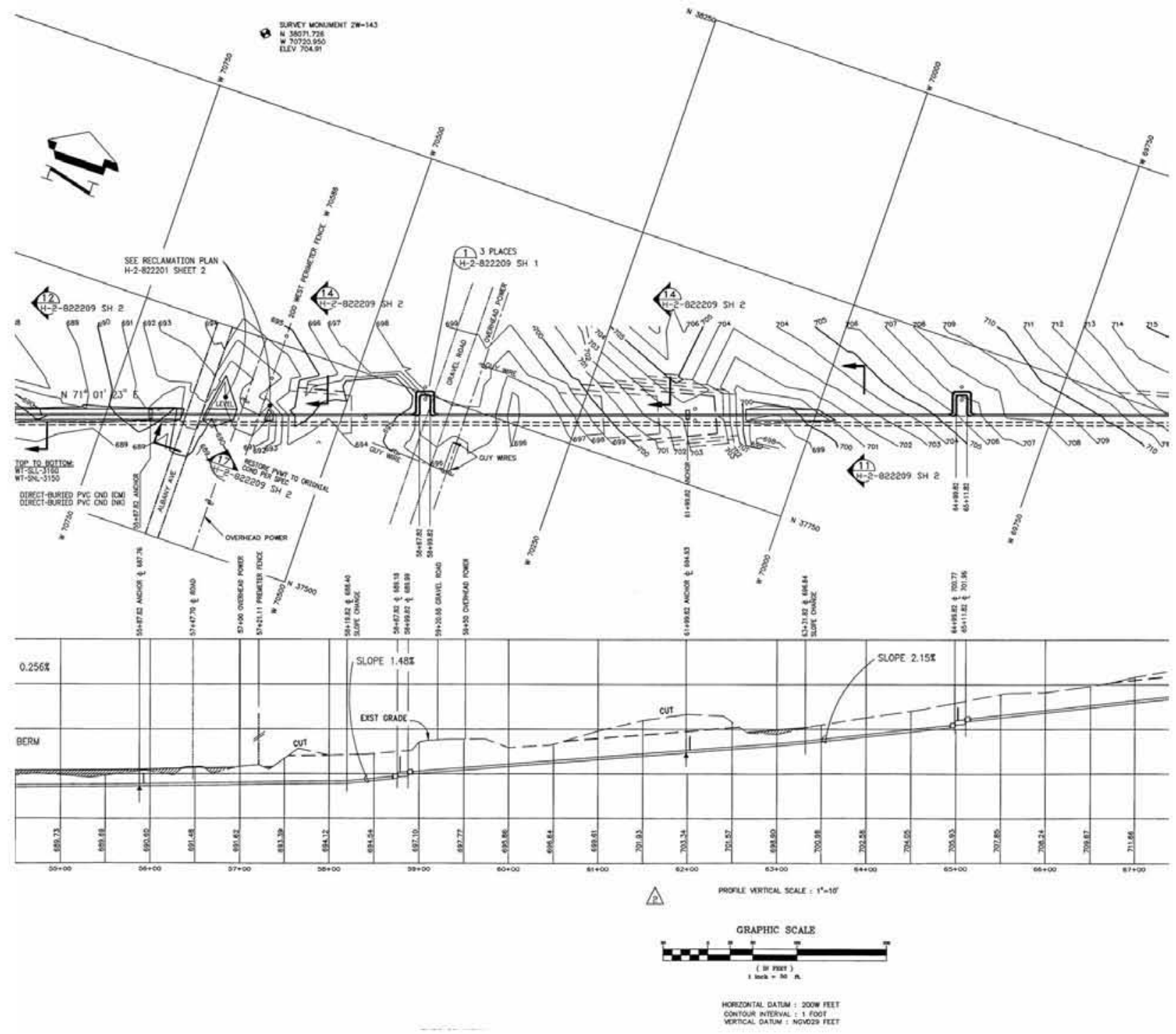

Source: H-2-822214, 1995, "Civil Plan and Profile STA 54+ to 72+," Sheet 1, Rev. 2.

Figure 3-3. Expansion Joint Layout for Cross-Site Lines ( 600-ft intervals) 


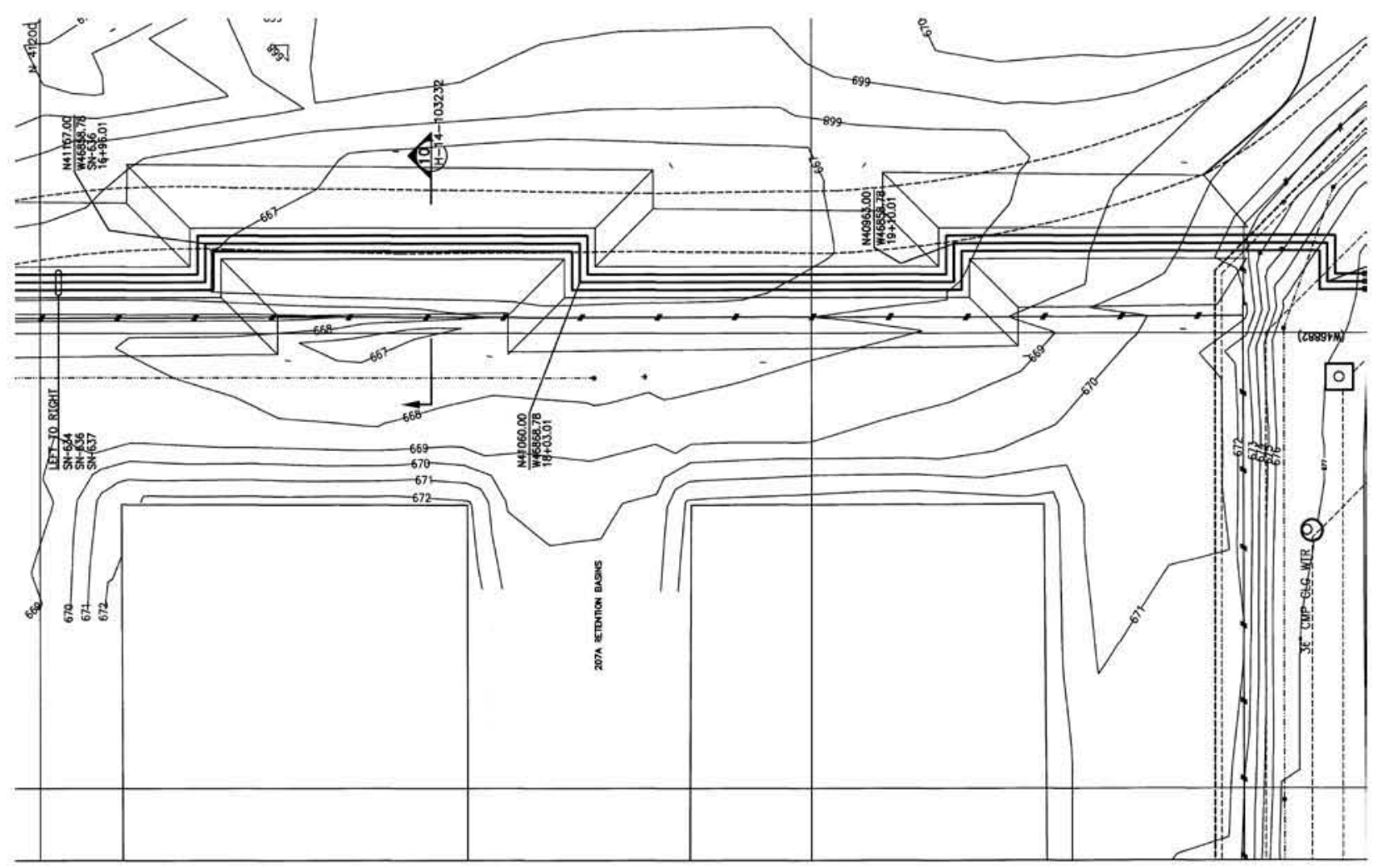

2. PLAN SN-634, SN-636 \& SN-637

Source: H-14-103240, 2004, "Civil SN-636, SN-637 \& SN-634 STA 14+25 to End," Sheet 1, Rev. 4.

Figure 3-4. Example of Pipeline Expansion Joints (100-ft intervals)

\subsection{KEY TRANSFER LINES}

As discussed in RPP-RPT-46112, Transfer Line Reliability for the Waste Feed Delivery Operations Research Model Phase 2, the cross-site transfer lines have a higher risk of failure and a higher consequence in terms of cost and schedule for replacement due to the line lengths. These transfer lines are essential for transfers from the 200 West Area to 200 East Area and if not operating when needed, will impact the overall WTP processing mission. More recently, DOE and the regulatory agencies have entered into discussions to determine how best to handle a leak in one of the DSTs from the primary containment into the secondary containment. One scenario that may be evaluated for the Tank AY-102 leak into the DST annulus may require pumping the tank and taking advantage of tank space in the SY Farm tanks located in the 200 West Area. This implies a potential transfer from the 200 East to 200 West Area in the nearterm. It also implies additional HLW solids transfers in the 200 East Area.

Lines less than 1,000 ft in length can conceivably be replaced with hose-in-hose aboveground transfer lines in the event of a plug or failure. The primary transfer lines from the WTP HLW and LAW feed tanks are also essential to supply feed to the WTP. However, the regulatory agencies recently informed DOE that they would not look favorably on any proposal to use hosein-hose transfer lines over very long distances. 
In RPP-RPT-51921, the current restoration time assumptions estimated are two years to replace a primary transfer line $(<1,500 \mathrm{ft})$, three years to replace a longer-length transfer line $(1,500$ to $4,000 \mathrm{ft}$ ), and four years to replace a transfer line longer than $4,000 \mathrm{ft}$.

Note: The cross-site slurry line, SLL-3160, has not been approved for use. During the upgrade of SLL-3160 (Project W-314, June 2005), an attempt was made to place a nitrogen blanket in the encasement between the primary and secondary lines to prevent the formation of condensation. It was determined that the encasement was incapable of holding pressure, and a problem evaluation was written to document this anomaly. Successful pressure testing of the encasement and readiness activities must be completed prior to using SLL-3160 (RPP-15136, System Design Description for the Replacement Cross-Site Transfer System Between 200 West and 200 East Tank Farms). Table C-1 of RPP-RPT-47572, Cross-Site Slurry Line Evaluation Report, documents a rough-order-of-magnitude estimate of $\$ 10 \mathrm{M}$ for a list of readiness items related to the SLL-3160 cross-site line.

The following are key findings from RPP-RPT-46112 and RPP-RPT-51921.

\section{SLL-3160 Restoration}

The cross-site slurry line, SLL-3160, is configured differently than the cross-site supernatant line SNL-3150, as SLL-3160 requires a booster pump to transfer slurry. A slurry transfer requires a higher flow rate in the line to maintain the critical velocity required to keep solids suspended during the transfer. The Phase 3.1 waste feed delivery (WFD) operations research (OR) model uses four years as the average restoration time for SLL-3160. Current contingency plans for a failure of the cross-site supernatant line, SNL-3150, are to use the cross-site slurry line, SLL-3160. The opposite is not true. There is no plan to retrofit the cross-site transfer system to enable slurry transfers on the supernatant line.

\section{Restoration of Plugged Transfer Lines}

To estimate a restoration for plugged lines, two subject matter experts were asked to provide a range of times based on their experience with such events. The subject matter experts estimated that from four to six months would be required to unplug a primary line. A restoration time of six months is used for conservatism in the Phase 3.1 WFD OR model for plugged lines.

\section{Transfer Line Restoration Time Implications}

The long restoration times that result from transfer line failures and plugging events are significant in terms of the WFD OR model, because when a failed transfer line cannot be bypassed, the model is unable to execute the waste transfer. The end result is that any transfer line failure that is unable to be bypassed greatly extends the mission life due to the long mean time to restore (MTTR) (typically years for a failed line) required for line restoration. 


\subsection{BACKGROUND}

\subsection{HISTORY OF PLUGGED LINES}

Waste transfer pipelines have plugged during past transfers of Hanford supernatant and slurry wastes. RPP-25113, Residual Waste Inventories in the Plugged and Abandoned Pipelines at the Hanford Site, documents the review of plugged pipelines taken out of service, with estimates of waste volumes remaining in those pipelines. The report provides a chronology of the plugging incidents in the cross-site transfer lines between installation in 1952 and replacement in the $1990 \mathrm{~s}$, and the line plugging incidents in the 200 East and 200 West Areas. Tables 7 and 8 of RPP-25113 summarize the pipeline failures and plausible plug types for the 1945 to 1972 time period. Most of the plugs were believed to be formed by cooling supernatant wastes of saturated or super-saturated solute solutions in buried pipelines, which initiated crystallization or precipitation of solids at various kinetic rates during the transfer operations. Under those transient conditions, the hydraulic characteristics of the increasingly viscous suspension with non-Newtonian yield stresses inevitably became laminar and led to pipeline blockage.

The plugs typically formed during transfer of supernatants of sodium-phosphate-rich metal wastes, aluminum-rich reduction-oxidation (REDOX) waste, and concentrated REDOX wastes from self-boiling tanks or various waste evaporator campaigns. The plugs were reported as hydrated minerals of carbonate and phosphate, such as the $\mathrm{UO}_{2} \mathrm{CO}_{3} \cdot 2 \mathrm{NaCO}_{3}$, and $\mathrm{Na}_{3} \mathrm{PO}_{4} \cdot 12 \mathrm{H}_{2} \mathrm{O}$ (or $\mathrm{Na}_{3} \mathrm{PO}_{4} \cdot 12 \mathrm{H}_{2} \mathrm{O} \cdot 1 / 4 \mathrm{NaOH}$ ), and aluminum precipitates from aluminum-rich REDOX wastes forming aluminum hydroxides $\mathrm{Al}(\mathrm{OH})_{3}$. The plugged REDOX waste lines were typically cleared with high-pressure water at ambient or elevated temperatures. As described in RPP-25113, further concentration of aged REDOX wastes in self-boiling tanks induced incidents of plugging. Pipelines were also plugged in the Tank U-107 saltwell pumping campaign in 2001 and 2002. Water was used in saltwell pumping and it is believed that a decrease in the supernatant hydroxide concentration, coupled with reduction in solution ionic strength, caused $\mathrm{Al}(\mathrm{OH})_{3}$ precipitation.

\subsection{NATURE OF PLUGS}

Potential pipeline plugging mechanisms for various Hanford waste streams, and the specific rheological and/or hydraulic problems associated with each plug, influence the performance of the selected unplugging technologies described in this section. The plugging mechanisms identified are based on the review of reports, memoranda, and other documents (listed in Table 4-1).

A pipeline plug may occur in the 2-in. slurry waste transfer lines containing undissolved solids or in the 3-in. supernatant/slurry transfer lines containing residual dissolved solids. The blockage formation is attributed to three distinct causes:

- Chemical instability (e.g., precipitation, crystallization)

- Settling of solids

- Hydrodynamic instability. 
Table 4-1. Hanford Pipeline Plugging Literature Survey Summary.

\begin{tabular}{l|l}
\hline \multicolumn{1}{|c|}{ Document number } & \multicolumn{1}{c}{ Title } \\
7S110-DLH-05-027 & 'Phosphate Solubility Under C Farm Retrieval Conditions" \\
\hline 24590-WTP-GPG-M-0059 & $\begin{array}{l}\text { Design Guide: Avoiding Chemical Line Plugging Plant Design } \\
\text { Considerations }\end{array}$ \\
\hline OR-78-88 & $\begin{array}{l}\text { Occurrence Report: Plugged Cross-Country Transfer Line } \\
\text { Studies }\end{array}$ \\
\hline ORNL/TM-2002/298 & $\begin{array}{l}\text { Cross-site Transfer System at Hanford: Long-term Strategy for Waste } \\
\text { Acceptance }\end{array}$ \\
\hline PNNL-11497 & $\begin{array}{l}\text { Recommended Responses to a Postulated HLW Feed Delivery Pipeline } \\
\text { Plug }\end{array}$ \\
\hline RPP-10905 & $\begin{array}{l}\text { Dilution and Flushing Requirements to Avoid Solids Precipitation and } \\
\text { Deposition During Tank Waste Transfers }\end{array}$ \\
\hline RPP-17247 & "Internal Letters Addressing Aluminum Solubility" \\
\hline RPP-17247, Appendix A & "Internal Letters Addressing Phosphate Solubility" \\
\hline RPP-17247, Appendix B & Waste Transfer, Dilution, and Flushing Requirements \\
\hline TFC-ENG-STD-26 &
\end{tabular}

Note: The complete reference for each document is provided in Section 11.0.

Chemical instability during the waste transfer changes the liquid phase composition and produces a solid phase. Phase transformations are induced by changes in the temperaturesolubility relationship between the solutes and solvent, variation in local concentration, or mixing of reactive wastes to promote crystallization or precipitation of solids. As solids are formed, the rheological and flow characteristic of the suspension changes, the flow in the pipeline transitions from turbulent to laminar, and plugging occurs. An increase in the suspended solids fraction promotes non-Newtonian characteristics and increases the apparent viscosity of the suspension. When slurries are transported, solids may settle when the flow rate is below the critical entrainment velocity and a sediment layer forms. Furthermore, hydrodynamic instability is induced when a flow transitions from turbulent to laminar or when changes in the flow pattern generate heterogeneous flow.

Chemical instability is the plugging mechanism associated with transferring supernatant waste. This plugging is caused by a decrease in temperature of the waste or by a change in solution electrolyte chemistry, which alters the solubility limits of certain constituents. Such instabilities may initiate crystallization or precipitation of solids from the liquid carrier and result in formation of an interlocking crystal network. This can develop a yield stress above the gelling point, causing flows to become laminar and form a blockage. Suspensions with an increasingly viscous character may also form and thereby increase the pressure drop as the suspended solids loading increases to the point that the slurry in transit plugs the pipeline. This crystallization or precipitation process occurs in phosphate- and aluminate-containing supernatant wastes. 
There are four plug-forming waste classifications that influence the transfer of supernatant wastes containing residual undissolved solids and slurry wastes, including:

- Phosphate-containing supernatant

- Aluminate-containing supernatant

- Concentrated, dissolved sodium nitrate and nitrite supernatant

- Solids-containing slurry.

The supernatant wastes containing high concentrations of phosphate are known to form interlocking $\mathrm{Na}_{3} \mathrm{PO}_{4}$, high aspect ratio needle-like crystals (shown in Figure 4-1), which form gels and impede the flow within the pipe as it cools, if the starting supernatant is inadequately diluted. Historically, lines that were plugged were abandoned because of phosphate formation near the gelation point. Useful information for assessing phosphate solubility is documented in RPP-17247, Dilution and Flushing Requirements to Avoid Solids Precipitation and Deposition during Tank Waste Transfers, Appendix B (Herting 1980). The letter included in the appendix of RPP-17247 provides solubility data for phosphate wastes at temperatures of $40^{\circ} \mathrm{C}, 50^{\circ} \mathrm{C}$, and $60^{\circ} \mathrm{C}$ and discusses data at $20^{\circ} \mathrm{C}$ at various dilution factors. In general, the crystallization of phosphate-rich

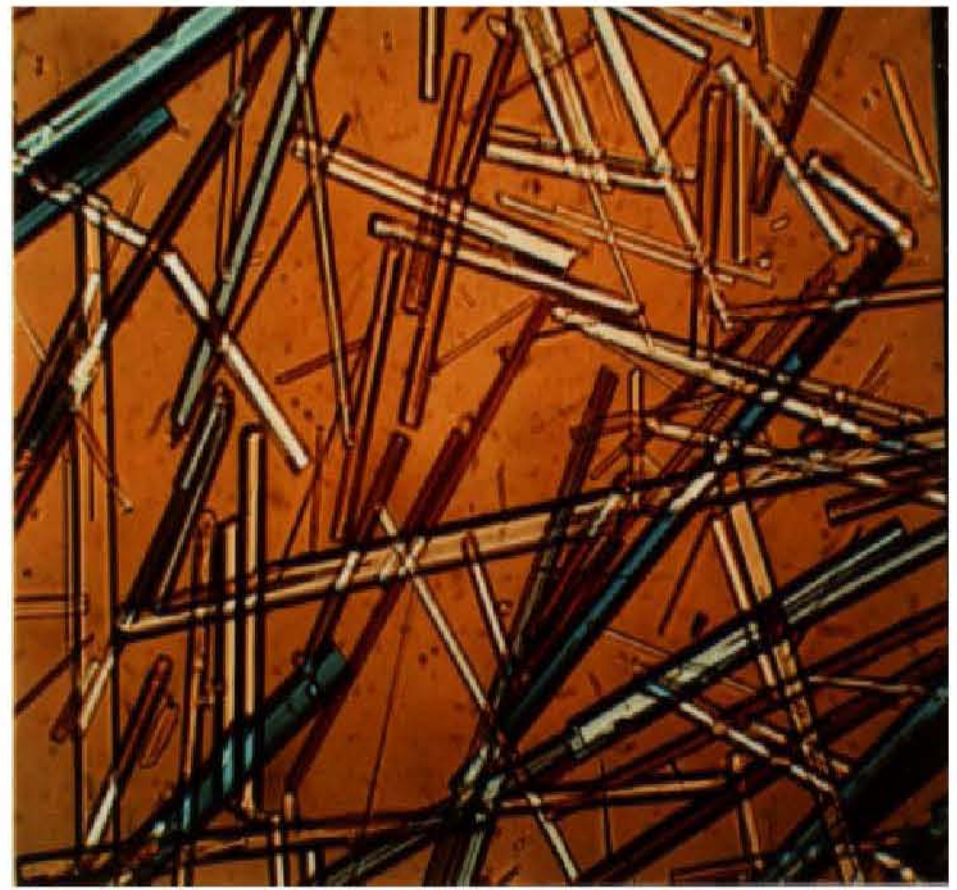

Source: RPP-17247, Dilution and Flushing Requirements to Avoid Solids Precipitation and Deposition during Tank Waste Transfers, Appendix B (Herting 1980).

\section{Figure 4-1. Photomicrograph of $\mathrm{Na}_{3} \mathrm{PO}_{4} \cdot 12 \mathrm{H}_{2} \mathrm{O}$} Crystals at $63 \times$ Magnification supernatant wastes is inhibited at high temperatures, when the solution is sufficiently diluted below the saturation limit of the operating pipeline transport temperature.

A phosphate plug can be removed if the pipeline unplugging technology provides adequate shearing force or induces sufficient vibration in the pipeline above the plug yield point. Supernatant waste containing high concentrations of the aluminate ion $\left[\mathrm{Al}(\mathrm{OH})_{4}{ }^{-1}\right]$ and polymerized forms of soluble aluminum found at high $\mathrm{pH}$ under high ionic strength condition may reach saturation limits (24590-WTP-RPT-PT-02-005, Flowsheet Bases, Assumptions, and Requirements). The saturation limits occur when these wastes are cooled in the pipeline or when there is inadequate dilution with water. Aluminum solubility correlations as a function of hydroxide concentration and solution temperature are presented in 24590-WTP-RPT-PT-02-005 below $1.2 \mathrm{~m}$ aluminum concentrations. 
In addition, aluminate ion solubility can be represented by the Barney Diagram on plots of $\mathrm{Al}^{3+}$ verses OH (TFC-ENG-STD-26). Both conditions decrease solution temperature and hydroxide ion concentrations and induce solid $\mathrm{Al}(\mathrm{OH})_{3}{ }^{5}$ precipitate formation. The presence of these precipitates increases the solids volume in the resulting suspension and thereby increases the potential for a pressure drop in the transfer line. A transition of flow from turbulent to laminar may arise as the suspended solids settle and plug the pipeline.

Solids formation may plug the lines if a post-transfer flush is not performed. The soluble salts in the supernatant waste can precipitate from residual line waste by water evaporation and adhere to the pipe surfaces. If there is significant salt precipitate, the possibility of a solids layer and hydrated solids buildup on the pipeline wall may obstruct the next transfer.

A last source of plug formation is by solids settling in the waste slurry pipelines. Much of the waste transfer analysis at Hanford has focused on this mechanism of pipeline plugging. The undissolved solids in a slurry waste may settle when the flow velocity is insufficient to keep them suspended, and a moving bed of particles begins to accumulate during a slurry transport operation. Settling solids in a moving bed of particles form a stationary bed that eventually fills the pipe and blocks flow, as transition from an unstable turbulent flow to a stable laminar regime occurs.

\footnotetext{
${ }^{5}$ There are at least five different forms of $\mathrm{Al}(\mathrm{OH})_{3}$ that differ from each other in crystal form rather than in chemical formula. These forms are gibbsite, nordstrandite, doyleite, bayerite, and amorphous aluminum hydroxide, in order of increasing solubility. Two different crystal forms of dehydrated hydroxide, both with the formula $\mathrm{AlOOH}$, are also known; these are boehmite and diaspore. These crystal forms are produced by high temperatures. The Boehmite formation occurs above $100^{\circ} \mathrm{C}$.
} 


\subsection{FUNCTIONS AND REQUIREMENTS}

Interfaces and functions for line unplugging using the pressure pulse technology in permanent and portable configurations are addressed in this section and summarized in Figure 5-1. Some of the functions are redundant to the flushing functions required for waste transfers. A permanent system has the potential to provide the functions required for both flushing and unplugging and also meet the requirements of HNF-4163, Double-Shell Tank Diluent and Flush Subsystem Specification. A portable system can be deployed and has similar functions and requirements as a hydrostatic test.

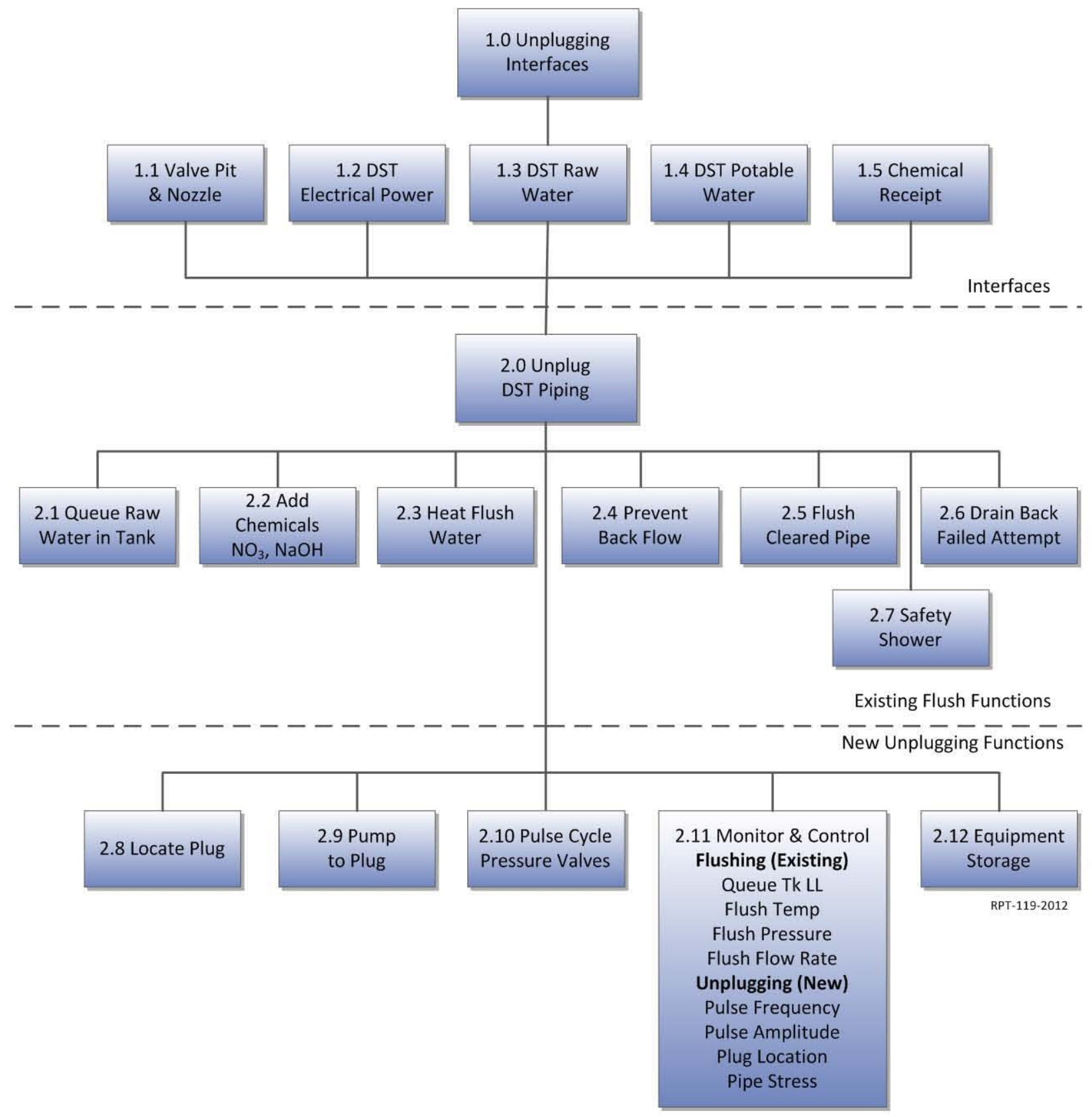

Figure 5-1. Line Unplugging Interfaces and Functions 


\subsection{UNPLUGGING INTERFACES}

Line unplugging at a valve pit involves the following functional interfaces with the tank farm and local services.

- Valve Pit and Nozzle (Interface 1.1). The waste transfer system piping originates in a below-grade concrete valve pit with a piping nozzle designed for use with a PUREX connector for remote operation with an overhead crane. The valve pit environment is typically spray-washed but contaminated such that personnel access is avoided if possible.

- Electrical (Interface 1.2). The unplugging system interfaces with the DST electrical power provided in the tank farms on an as-needed basis, including 480/277 VAC threephase power and 240/120 VAC and 208/120 VAC three-phase and single-phase power, as required.

Basis: HNF-4163, Section 3.1.2.1.1

- Raw Water (Interface 1.3)

- The DST diluent and flush subsystem and unplugging system shall be capable of receiving strained water at a continuous flow rate of at least $220 \mathrm{gal} / \mathrm{min}$ and a minimum pressure of $80 \mathrm{lbf} / \mathrm{in}^{2}$ gauge.

- The DST diluent and flush subsystem and unplugging system shall be capable of receiving raw water strained to the extent specified in HNF-4157, Double-Shell Tank Utilities Subsystem Specification, Section 3.2.1.2.c.

Basis: HNF-4163, Section 3.1.2.1.2

- Double-Shell Tank Potable Water (Interface 1.4). Potable water shall be provided for an integrated safety shower for the permanent unplugging system. A portable safety shower shall be provided for the portable unplugging system.

- Chemical Receipt (Interface 1.5). Caustic and sodium nitrate will be received for use in inhibited water for corrosion or to reduce the surface tension of the water for unplugging. Provisions for the addition of other chemicals to facilitate dissolution of solids in the piping at the flushing stage will also be considered.

\subsection{UNPLUGGING FUNCTIONS}

The following summarizes the basic unplugging functions for a pressure pulse system.

- Queuing Raw Water in Tank (Function 2.1). A queuing tank shall be sized to provide a buffer between the raw water supply and the unplugging process equipment. The tank will be equipped with agitation and heating for manually adding chemicals for the portable application. 


\section{- Add Chemicals $\mathrm{NO}_{3}, \mathrm{NaOH}$ (Function 2.2).}

- Unplugging Chemicals - Corrosion is not a high priority in an unplugging event. However, addition of sodium nitrite to reduce surface tension in the raw water is warranted to improve the chances of infiltrating a plug. The provision to add other chemicals to assist in the dissolution of a plug may also be necessary depending on the nature of the plug.

- Inhibited Water - If a flush is required, the following compositions should be used:

a. For carbon steel piping, flushes shall consist of inhibited water, which contains at least $0.01 \mathrm{M}$ hydroxide and $0.011 \mathrm{M}$ nitrite.

b. For stainless steel piping, flushes shall consist of raw water, deionized water, or ionic species that promote the formation of an oxide layer on the pipe surface (e.g., nitrate, phosphate, aluminate). If an ionic solution is used, the species should have a concentration of $0.1 \mathrm{M}$.

Basis: TFC-ENG-STD-26, Section 3.7.3 (2)

- Receiving Chemicals - Chemical unloading equipment is expected to include connections to a source of concentrated liquid sodium hydroxide and sodium nitrite, transfer lines, mixing equipment, measurement instruments, and metering pumps to permit addition of the chemicals to the diluent and flush solutions.

Basis: HNF-4163, Section 3.7.3

The subsystem components shall be designed to perform their intended functions in the chemical environments defined in the following requirements.

a. The DST diluent and flush subsystem equipment shall be capable of receiving and diluting concentrated sodium hydroxide solutions obtained from a supplier that are up to $50 \mathrm{wt} \%$.

b. The DST diluent and flush subsystem equipment shall be capable of receiving and diluting concentrated sodium nitrite solutions obtained from a supplier that are up to $40 \mathrm{wt} \%$.

Basis: HNF-4163, Section 3.2.5.2.1

- Heat Flush Water (Function 2.3). Raw water used to unplug or flush a cleared line may be heated up to the transfer line design temperature (usually $200^{\circ} \mathrm{F}$ ) to aid in removing and dissolving the plug.

- Prevent Backflow (Function 2.4). The piping system shall be designed to prevent backflow that would contaminate the unplugging system equipment (e.g., pump, pulse valves, queuing tank). 


\section{- Flush Cleared Pipe (Function 2.5)}

- Flushing Supernatant Lines - Flushing after an unplugging event mirrors the flush protocol after a typical transfer summarized below.

a. Flushing shall be conducted with raw water or preferably with inhibited water. If routinely available in the tank farm or via the water truck/skid, inhibited water is used for flushing.

b. Since most components are more soluble at higher temperature, the use of hot water for flushing is preferred.

Basis: TFC-ENG-STD-26, Section 3.7.1

- Flushing Slurry Lines - A minimum flush velocity of $6 \mathrm{ft} / \mathrm{sec}$ is targeted, which should be adequate to clear $80 \mu$ particles with a specific gravity of 3.0 from a 3 -in. line. Lower velocities may be used if critical velocity calculations, performed in accordance with TFC-ENG-STD-26, Section 3.6, indicate a lower velocity is adequate to resuspend the anticipated solids in the waste or if the transfer did not contain solids and the flush is being used to remove soluble scale from the lines.

Basis: TFC-ENG-STD-26, Section 3.7.2

- Flush Volume - The flush volume shall be equal to a minimum of 1.5 line volumes. Unless a specific restriction or line plug is known or suspected, the total volume of flush water used shall be minimized to preserve DST space.

Basis: TFC-ENG-STD-26, Section 3.7.2 (3)

- Drainback Failed Attempt (Function 2.6). In the event the unplugging function fails, the flush solution introduced into the line must be drained back. The jumper and valve arrangement shall enable this drainback to be routed to the DST or pit drain.

- Safety Shower (Function 2.7). Handling chemicals in manual or automated systems implies a need for a safety shower and an eyewash station. A permanent facility would have an integrated shower and eyewash station. A portable system would use an existing safety shower and eyewash or provide a temporary unit if chemicals are involved.

- Locate Plug (Function 2.8). Transfers often require multiple lines with connections through multiple valve pits. When a plug occurs, it may not be obvious where the plug is or which line is plugged. Individual portions of the route would be flushed to determine which specific line is plugged. For a portable system, the unplugging equipment would be connected to the plugged portion, and pressure pulsing would be applied.

- Pump to Plug (Function 2.9). A high-pressure pump shall provide the pressures needed to clear a line. The pump shall be capable of pressures of at least $3000 \mathrm{psig}$. A means of capping the maximum pressure shall be provided via a pressure relief system to recycle water back to the queuing tank. The maximum pressure shall be variable depending on the unplugging strategy used for a particular pipeline and plug combination. The pump is provided by the unplugging vendor for the modular system. A flush pumping capability shall also be provided to clear the line once the plug is broken free. This requirement may involve a second pump. 
- Pulse Cycle Pressure Valves (Function 2.10). A high-frequency pulsing valve shall be used to manage the pulse wave amplitude and frequency. Solution drainback from the valve pulsing shall be drained to the tank or retained in the system. Drainback from the valve shall not be recycled to the queuing tank to avoid contaminating the unplugging equipment.

- Monitoring and Control (Function 2.11). The monitoring and control functions would be fully integrated with the monitoring systems of a permanent facility. The portable facility shall have local monitoring and control features.

- Equipment Storage (Function 2.12). The pressure pulse equipment shall be stored to achieve protection from weather damage. Routine maintenance and calibration of the equipment shall be provided while it is not in use such that immediate activation for unplugging is possible by Operations.

Table 5-1 summarizes the interfaces and functional requirements. 
Table 5-1. Unplugging Functional Requirements (3 pages)

1.0 Interface with the existing The unplugging system will interface with the existing pits and DST farm infrastructure utility interfaces.

1.1 Valve pit and nozzle interface

The valve pit cover blocks will be removed or pit access will be provided through existing flush routes.

The cover block will be left in place for confinement or a

2- and 3-in. nozzles are normally accessed with PUREX

cture will be built over connectors, which have a working pressure of $400 \mathrm{psig}$ and are The fitting used to access the PUREX pipe nozzle shall used up to $600 \mathrm{psig}$ for pressure testing. Another fitting may be withstand the unplugging pressures deployed (the maximum required for higher pressures.

1.2 Connect to DST electrical Electrical power is supplied in the tank farms for flushing lines pressure allowed)

powe and can also be used for unplugging.

Electrical power shall be extended from existing sources within the DST system to supply the unplugging system electrical requirements.

1.3 Connect to existing DS raw water supply

Raw water is supplied in the tank farms for flushing lines an can also be used for unplugging.

1.4 Connect to existing Cotable water for safety potable water for safety

A safety shower/eyewash station should be provided unless already exists in the proximity of the unplugging equipmen and chemical makeup.

1.5 Provide means and location for receiving chemicals

The unplugging system will be designed for manual or automated addition of chemicals. Caustic and sodium nitrit will be received totes at commercial strength and diluted wi raw water to inhibited water concentrations.

An unplugging system will be permanently incorporated into the diluent and flush system or provided as a portable system
The unplugging system shall be designed to receive water from the existing DST raw water supply. During operations for pulsing, the raw water may need to be physically disconnected for safety reasons.

Potable water shall be provided for an integrated safety shower and eyewash station for the permanent system. A portable safety shower and eyewash station shall be provided for the portable unplugging system.

The unplugging system shall be capable of using concentrated commercial-grade sodium hydroxide $\quad$ c. The DST diluent and flush subsystem shall be capable of using concentrated (con to 50 concentration of subsystem.

The unplugging system shall be capable of using concentrated commercial-grade $40 \mathrm{wt} \%$ sodium nitrite provided by the DST maintenance and recovery subsystem.

An unplugging system will be permanently incorporated into the diluent and flush system or provided as a portable system. Initiation of the unplugging system shall be as soon after the identification of the plugged condition as possible. The plugged line will not be drained to avoid introduction of an
air pocket that insulates the plug from the liquid pulse head.

HNF-4163, ${ }^{\mathrm{a}}$ Section 3.2.1.
Requirement source

HNF-4163, ${ }^{\mathrm{a}}$ Section 3.1.2.1.1

The DST diluent and flush subsystem shall be capable of receiving 480/277 VAC three-phase power, 240/120 VAC and 208/120 VAC threephase and single-phase power, as required.

The DST diluent and flush subsystem shall be capable of performing its functions, as specified herein, using the power allocated by the DST electrical power system.

HNF-4161, ${ }^{\text {b }}$ Section 3.1.2.1.3

b. The DST transfer piping subsystem shall be designed to allow access to raw water from the DST raw water sub-element for pipe unplugging, flushing, and decontamination.

HNF-4163, ${ }^{\text {a }}$ Section 3.3.6.1

Safety shower and eyewash stations shall be provided at the diluent and

clush stitons and

commercial-grade sodium hydroxide (commonly referred to as caustic soda) at a concentration of up to $50 \mathrm{wt} \%$ provided by the DST maintenance and recovery subsystem.

The DST diluent and flush subsystem shall be capable of using concentrated commercial-grade $40 \mathrm{wt} \%$ sodium nitrite provided by the DST maintenance and recovery subsystem.

HNF-4163, ${ }^{a}$ Section 3.2.4.b

A single replaceable component of the DST diluent and flush subsystem should be designed to enable replacement within 8 hours. This time does no include logistics or administrative delay time. 
Table 5-1. $\quad$ Unplugging Functional Requirements (3 pages)

A queing tank will be provided to support the heating, and rates with the raw water supply.

2.2 Add chemicals $\mathrm{NO}_{3}$ $\mathrm{NaOH}$

2.3 Heat flush water

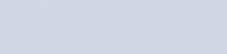

\subsection{Prevent backflow}

The pulse and flush systems are protected from backflow with contaminated solution from the plugged pipe. corrosion protection of the piping. For unplugging, the prim concern is to reduce the surface tension of the water to penetrate the plug. Adding chemicals to clear a line after the plug is dislodged is primarily a flushing function but it should be integrated with unplugging.

An option to use heated water to unplug or flush the line will be provided.

A centrifugal pump is provided to clear a plug that had been moved by the high pressure pump.

Provisions will be made to drain the line for pulse down and in

2.6 Drainback failed attempt/receive pulse down

2.7 Provide safety shower

A safety shower/eyewash station should be provided unless on
Provisions will be made to drain the line for pulse down and in Drainback from relief of a pressure pulse or to drain the line the event of a failure to unplug the line. This drainback will not shall be routed to the DST in combination with a means of contaminate the unpluging system.

backflow prevention to avoid contaminating the unplugging equipment. already exists in the proximity of the unplugging equipment and chemical makeup.

Potable water shall be provided for an integrated safety
The unplugging shall provide a flushing capability at

$10 \mathrm{ft} / \mathrm{sec}$ and a volume equivalent to twice the volume of transfer line.

Features to flush with inhibited waster shall be provided. The provision to add other chemicals to assist in the dissolution of a plug may also be necessary depending or nature of the plugging event.

Raw water used to unplug or flush a cleared line may be heated up to the transfer line design temperature (usually $200^{\circ} \mathrm{F}$ ) to aid in removing and dissolving the plug and flushing the line.

The piping system shall be designed to prevent backflow that would contaminate the unplugging system equipment (e.g., pump, pulse valves, queuing tank).

A second pump shall be provided to flush the line after unplugging at a flow rate corresponding to $10 \mathrm{ft} / \mathrm{sec}$ for two line volumes. (2)

Requirement source

HNF-4163, ${ }^{\text {a }}$ Section 3.2.1.1.g

The DST diluent and flush subsystem shall be capable of flushing each waste transfer line with a volume of flush solution that is twice the volume of the transfer line.

HNF-4163, ${ }^{\text {a }}$ Section $32.11 . \mathrm{h}$

The DST diluent and flush subsystem shall be capable of providing a flow of $10 \mathrm{ft} / \mathrm{sec}$ to the DST transfer valving subsystem independent of the waste transfer pump at a pressure no greater than the existing system design pressure. This flow rate applies to slurry transfers $(\geq 3 \mathrm{wt} \%$ insoluble solids) for resuspension of settled solids.

HNF-4163, ${ }^{a}$ Section 3.7 .3

Chemical unloading equipment is expected to include connections to a source of concentrated liquid sodium hydroxide and sodium nitrite, transfer lines, mixing equipment, measurement instruments, and metering pumps to permit addition of the chemicals to the diluent and flush solutions.

HNF-4163, ${ }^{a}$ Section 3.7 .2

Diluent or flush solution is heated to reach a preset temperature if necessary for a given application. The preset temperature is dependent on the specific ank waste characteristics and the application of the diluent or flush solution. Heating equipment includes the heater-whether it is electric, fossil fuel, or steam - the tank or containment where the diluent or flush solution is to be heated, valves, and measurement instruments.

HNF-4160, ${ }^{\mathrm{d}}$ Section 3.2.1.8

The DST transfer valving subsystem shall be capable of preventing any backflow of waste or contaminated flush or diluent from the transfer pump int the raw water, flush water, or diluents supply piping.

a. The backflow prevention device shall comply with the requirements of WAC 246-290.

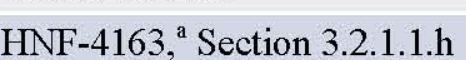

The DST diluent and flush subsystem shall be capable of providing a flow of $10 \mathrm{ft} / \mathrm{sec}$ to the DST transfer valving subsystem independent of the waste transfer pump at a pressure no greater than the existing system design pressure. This flow rate applies to slurry transfers ( $\geq 3 \mathrm{wt} \%$ insoluble solids) for resuspension of settled solids.

HNF-4161, ${ }^{\mathrm{b}}$ Section 3.3.8.

b. Primary piping and encasements shall be fully drainable.

HNF-4163, ${ }^{a}$ Section 3.3.6.1

shower and eyewash station for the permanent system. A A safety shower and eyewash stations shall be provided at the diluent and flush portable safety shower and eyewash station shall be provided stations and shall comply with ANSI/ISEA Z358.1.
for the portable unplugging system. 
Table 5-1. Unplugging Functional Requirements (3 pages)

Function

$2.8 \quad$ Locate plug

Pump to plug

Pulse cycle pressure

valve

2.11 Monitor and control

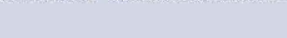

Equipment storage
Description multiple valve pits. When a plug occurs, it is not obvious where the plug is or which line is plugged. Individual portions of the route would be flushed to determine which specific line is plugged. The unplugging equipment would be connected to the plugged portion and pressure pulsing would be applied.

A high-pressure, low-flow pump is provided to unseat the plug at the maximum design pressure of the pipe. The system should provide flexibility in applying different pressures for different applications.

A pulse valve system is provided to vary the static pressure by up to $100 \mathrm{psig}$. The pressure and pulsing are varied from 200 to $1,500 \mathrm{psig}$ in static pressure with pulses that vary up to $100 \mathrm{psig}$ from the static pressure.

Typical monitoring and control features will include:

- Queue tank liquid level, temperature

- Flush temperature, pressure, flow

- Pulse frequency, amplitude

The pressure pulse equipment shall be stored to achieve protection from weather damage. Routine maintenance and calibration of the equipment shall be provided while it is not in by Operations.

\section{Requirement}

Depending on the routing, when the plug occurs, a decision shall be made to attempt to clear the entire route or flush individual segments of the route and focus the unplugging operation on one single line.

A piston pump shall be provided with a pressure range of 200 to $5000 \mathrm{psig}$ with a $20 \mathrm{gal} / \mathrm{min}$ flow rate.

The pulse valve provides a pressure surge of up to $100 \mathrm{psig}$ with a drainback of contaminated solution into the DST.

The monitoring and control functions shall be fully integrat with the DST monitoring systems for a permanent facility. The portable facility shall have local monitoring and control features.

Weather protection, routine maintenance, instrument calibration, and operator training shall be provided such that unplugging can be conducted without delays.
Requirement source

TBD - Requirement is a candidate for addition to HNF- 4163 .

\section{Specifications from vendor experience.}

HNF-4161, ${ }^{\text {b }}$ Section 3.2 .7

The design shall, to the maximum extent practicable, provide sufficien flexibility to accommodate programmatic changes or operational modifications.

Specifications from vendor experience.

\section{NNF-4163, ${ }^{\text {a }}$ Section 3.7.}

A typical instrumentation and control system comprises four major subsystems:

- Sensors, which provide electrical or mechanical analogs of operating variables

- Telemetry, which provides for the transfer of sensor information to a central collection point

- Central control, which provides for the interrogation of sensors, the recording and processing of sensor outputs, and the routing of processed data to displays or storage

- Displays, which present processed data in a convenient form. Instrumentation is installed on the DST diluent and flush subsystem to provide safe and reliable operation.

Similar to principles covered in HNF-4159. ${ }^{\mathrm{f}}$ 


\subsection{KEY FUNCTIONAL ISSUES}

A technology that induces pressure pulses to unplug a pipeline also has a number of flushing functions that are necessary for normal operation. Some of these functions include heating raw water, conditioning it with dilute sodium hydroxide and sodium nitrite, and pumping the flush water up to $400 \mathrm{psi}$ (maximum allowed pressure rating). The flushing system can be used to preheat the line before a transfer and flush it after the transfer.

In the event of a plugged line, waste pumping is suspended and a pressurized flush is attempted at the allowed line pressure to try to unplug the line. Although some cross-site transfer lines are rated up to $1,490 \mathrm{psig}$, there are components in the system (e.g., PUREX connectors) rated at 400 psig that limit pressure. The pressure pulse technology essentially cycles two valves to apply and relieve pressure from 0-180 cycles per second (according to the patents), thereby creating adjustable pressure waves to the point of cavitation in the pipeline to dislodge the plug. The Hydrokinetics field experience uses 2-30 cycles per minute with manual operation to achieve consistent success in unplugging lines over short and long distances. The variables include the flush solution pressure, magnitude of the pulse pressure, and frequency.

Application of the pressure pulse technology introduces a number of key issues, which are summarized in the following paragraphs.

Air Pockets. The presence of an air pocket reduces the effectiveness of pressure pulsing. The transfer lines slope up or down from the valve pits. Where the transfer lines slope up to a plug location, an air pocket is formed when the transfer is suspended and the transfer line is drained. The trapped air insulates the plug from contact with water and the vibration effects of the pulse action. Pulling a vacuum on the pipe would reduce but not eliminate the air pocket. There are three possible solutions for this issue:

1. A line plugging incident is not instantaneous. Plug incidents are generally preceded by a gradual buildup of solids, and the flow rate/line pressure reflects the change over several hours. Procedurally, the operational response is to add dilution water from the flush system. If the line still plugs, there is a good chance that the line will be filled primarily with flush water rather than supernatant or slurry that could settle more solids. This scenario allows the pressurization and pulsing procedures to begin immediately without draining the line. It requires the features to be built into the flushing system functions so that a jumper change is not required.

2. Line plugs are not necessarily airtight. Over time, the air pocket may pass through the plugged portion of the line, allowing flush solution to come in direct contact with the plug.

3. For lines other than the cross-site transfer lines (SNL-3150 and SLL-3160), the slope is in one direction between pits. The transfer line either does not drain from the upper end (remains full after the transfer) or it is refilled slowly (channel flow) to displace the air pocket. If the cross-site transfer lines are drained before unplugging begins, the refilling process entails exhausting the air pocket through the vent lines at the $624 \mathrm{~V}$ vent station (Figure 5-2) hydraulic high point. The vent station is intended to vent air into the line; using the pit in the present configuration requires special administrative controls or a redesign to prevent contaminating the pit with exhaust air from the transfer lines. 
Study Premise: Both scenarios are addressed (with and without an air pocket).

Pressure Limitations. The pulse technologies work at any pressure but performance can be improved at higher pressures (1,000 psi and above). The cross-site transfer line is designed for 1,490 psig, but there are weaker components (PUREX connectors) in the system that limit the pressure to $400 \mathrm{psig}$ or less. Design modifications may be possible to increase the maximum pressure, but the pressure pulse

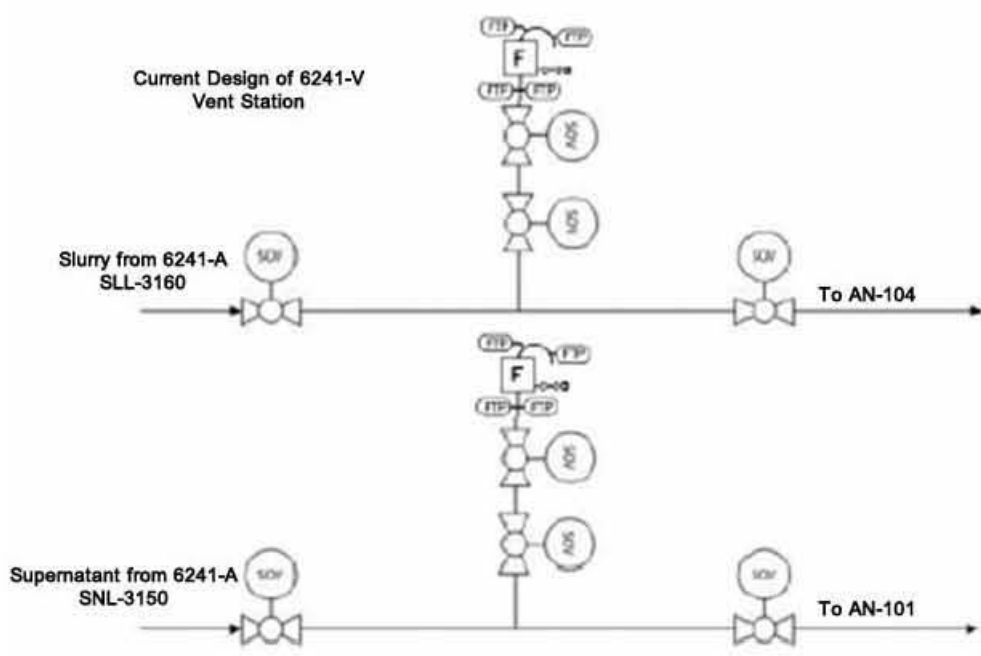

Figure 5-2. 6241-V Vent Station technology shows enough promise to proceed within the limitations of the current system.

Study Premise: Pulsing at pressures within the constraints of the limiting components in the piping system will be considered (275-400 psig). Removing those components and using the pipe design pressure will also be considered (1,000-1,490 psig).

Structural Damage. Introducing pressure pulses at varying frequencies runs the risk of setting up a standing wave that matches a natural harmonic in the piping system and causing excessive vibration to structural components and damage to the line. Alternatively, the expansion joints may absorb the force of the standing wave in nearly incompressible fluids in the pipe.

Flow-Induced Vibration. Vibration is addressed as a topic for fitness-for-service. Consistent with process and power industries practice, the waste transfer lines were not originally designed for flow-induced vibration (FIV). FIV could result from pressure pulsing in the line or vortex shedding and may be amplified by acoustic resonance. There have been no visual or instrumented inspections specified for FIV of the jumpers; however, during camera inspections for leak check of the jumpers, no evidence of vibration has been visible (RPP-RPT-52206, Tank Farms Waste Transfer System Fitness-for-Service Requirements and Recommendations, Section 6.1.1.5)

Two actions can be taken to address pressure pulse-generated structural damage:

1. Analysis - Some of the 200 East Area piping routes have been structurally analyzed to address the potential for water hammer, a closely related phenomena. One or all of these lines can be modeled through the range of frequencies to address both damage and dampening.

2. Physical monitoring - During physical monitoring, the pressure, frequency, and amplitude are gradually increased. It may also be possible to monitor the vibration in the line directly with an accelerometer as a means to detect excessive vibration during unplugging. Additionally, vendor field experience with similarly configured piping with expansion joints may provide insight on the potential for structural damage. 
Study Premise: The study will address these options and recommend one or more.

Is the Pulsing System Portable or Permanent? A portable system could be deployed with jumper modifications to avoid changing jumpers and draining the line. A permanent system has the advantage of being built into the flushing functions specified in HNF-4163 for immediate deployment after a line plug incident. However, permanent flush systems are costly. The first permanent flush system is currently planned to cover the AY and AZ Farm tanks, and three follow-on projects are planned for AW, AP, and SY Farm tanks. These projects could be modified in scope to include the pressure pulsing function and cover the most important transfer routes. The remaining routes would require a portable system and would encounter the delays associated with deploying the system in a nuclear environment.

Study Premise: The study considers both options. A final decision is beyond the scope of the current study.

Pulsing from One Side or Both Sides. FIU has conducted recent pressure pulse work from both ends of a plugged pipe via "asynchronous pulsing." Pressure pulses are created asynchronously to break the mechanical bonds between the blockage and the pipe walls as a result of the standing waves from each end. This approach has shown some success in testing at FIU, but it has not been applied in the field by a vendor so there is no operational history. The approach is also sensitive to air pockets attenuating the force of the pulse. Pulsing from both ends of a plugged line implies twice the infrastructure and project costs.

Study Premise: The study will consider asynchronous pulsing as the same pulse technology, but in a demonstration phase of development. 


\subsection{ALTERNATIVE TECHNOLOGY EVALUATION}

The following subsections provide a fundamental description of the Hydrokinetics and PipePulse technologies to unplug pipelines. Deployment of the two technologies in the Hanford waste transfer system is also evaluated.

Potential modifications and the support system required for operation of the Hydrokinetics technology in the waste transfer system and the potential adverse impacts of deployment in the context of fitness-for-service evaluation criteria and ALARA (as low as reasonably achievable) principles are discussed.

An overview of the Pipe-Pulse technology is also provided. It is important to note the scope of the Pipe-Pulse evaluation and the analysis for use in the Hanford waste transfer system was limited because of a strict nondisclosure agreement required by Paradigm Flow Services for engagement in technical discussion on the Pipe-Pulse technology efficacy. Thus, evaluation efforts are focused on the Pipe-Pulse patent application filing and public domain literature.

\subsection{PULSATION FUNDAMENTALS}

The principal of operation for the Hydrokinetics and Pipe-Pulse technologies to unplug pipelines is based on applying pressurized fluid pulsation to remove blockage or, at a minimum, create an aperture in the plug to restore flow in the pipeline. These fluctuations in pressure travel through the pipeline as acoustic waves. Pulsation is achieved by pumping raw water from a storage vessel using a regulated high-pressure pump and transferring it in the pipeline with a controllable cycling valve system. At any point in the flowing fluid, a distinction exists between the fluctuating parameters and the steady-state parameters. The steady-state parameters of a fluid are flow rate and static pressure distribution along the pipe length. When pulsation occurs, the fluctuating pressures and flows are superimposed on the steady-state values. In this case, the pressure at a given point can be expressed as:

$$
P(t)=P_{S S}+P_{\text {cyclic }} \times \sin (\omega t) \quad(\text { Equation } 1)
$$

Where:

$$
\begin{aligned}
& P(t)=\text { pressure at a given point as a function of time } \\
& \mathrm{P}_{\mathrm{SS}}=\text { steady-state pressure } \\
& \mathrm{P}_{\text {cyclic }}=\text { fluctuating pressure or pulsation } \\
& \omega=\text { angular frequency } \\
& \mathrm{t}=\text { time. }
\end{aligned}
$$

When a pipeline plugs, the fluid flowing at a steady-state flow rate decelerates to zero velocity and the steady-state pressure is the static pressure in the pipeline. The focus of both the Hydrokinetics and Pipe-Pulse technologies is on varying modes of the fluctuating pressure parameter of Equation 1 to attempt unplugging the pipeline. This is explained further in the following subsections. 
When pressure pulsations occur in the piping system, they propagate through the fluid as acoustic waves (or longitudinal mechanical waves) and move in fluid as traveling waves. The traveling waves have properties of frequency, amplitude, wavelength, and velocity. For any given fluid at a given temperature and pressure, all disturbances travel at the speed of acoustic velocity. ${ }^{6}$

The acoustic velocity determines the rate at which a pressure disturbance (pulsation) travels in fluid. Water or sodium hydroxide solutions of varying molarity are the main fluids used in pressurized pulsation in unplugging transfer lines at Hanford. ${ }^{7}$ Caustic solution may be used when the line blockage is from aluminum-containing precipitation. A typical acoustic velocity for water (sea water at $25^{\circ} \mathrm{C}$ ) is $5,023 \mathrm{ft} / \mathrm{sec}$ (CRC Handbook of Chemistry and Physics, p. E-47 [Weast 1974-1975]). The fluid acoustic velocity decreases as the fluid compressibility factor changes in the system. Any flexibility in the pipe wall lowers the acoustic velocity, and the presence of entrained gas induces additional compressibility that drastically reduces the fluid acoustic velocity. For instance, introduction of approximately 0.1 percent by volume air can decrease the fluid velocity by half.

An acoustic wave traveling in a pipe is reflected when changes in the flow area occurs. Physical elements that generate reflections include closed ends, open ends, changes in the pipe diameter such as expansion and contraction, branches, tees, and flow restrictions. It is assumed no change in flow area from various physical elements occurs when pressure pulsation is used because the slurry and supernatant wastes are transferred respectively in 2- or 3-in. diameter Schedule 40 stainless steel pipes in the Hanford waste transfer system. In this case, the incident wave from the initiated pressure pulse as it reaches the pipeline blockage will be the dominant reflection. The incident and reflected waves have the same frequency, and the superposition of these traveling waves generates a standing wave containing antinodes and nodes. ${ }^{8}$

Besides the formation of a standing wave as the traveling wave reaches the blockage and is reflected, an acoustic resonance can form when the wave frequency and the plug location within the pipe length are such that the reflected wave arrives at the source at the same time as the new incident wave is being generated. Under this acoustic resonance condition, the amplitude of the standing wave can quickly grow to an extremely large value. The acoustic resonance has the potential to become problematic when the frequency of pulsation matches one of the resonant frequencies of the transfer line system and the pressure and displacement amplitude build up to a destructive value. A piping system contains acoustic damping by losses within the piping from pipe friction, bends, and restrictions that reduce acoustic resonances. A detailed pipeline stress analysis can provide insight regarding the prominence of acoustic resonance as a pressure pulsation technology is applied in the Hanford waste transfer system.

In general, the standing wave creates continuous pressurized fluid vibration at the interface between the pipeline and blockage that transfers pulsation energy to the pipe wall and blockage.

\footnotetext{
${ }^{6}$ An acoustic wave is composed of a pressure wave and a velocity wave in 90 -degree phase difference. Pulsation can be described by either wave; however, there are more particles to focus on the pressure wave in the context of pressure pulsation.

${ }^{7}$ In industrial application of the Hydrokinetics and the Pipe-Pulse technologies, water is typically the main fluid used. However, a number of chemicals and solvents are used to breakdown inorganic and organic blockage.

${ }^{8}$ In contrast to the traveling wave, the pulsation amplitude for all particles is not the same in a standing wave and varies with particle location.
} 
The blockage and the pipe wall vibrate at different frequencies because of differences in inherent characteristics, thereby breaking the bond between them that allows fluid to penetrate, forming an aperture and dislodging the blockage from the pipe wall. The dislodged blockage is then removed by restored turbulent flow and may re-dissolve or re-entrain solids in the transport line. The induced vibration by the standing wave also provides a steady shear field in the yield shear thinning (thixotropy) suspension. Such suspensions exhibit gelation behavior similar to the interlocking crystalline network produced from the interaction between high-aspect ratio phosphate containing crystals and the liquid phase (see Section 4.2). As the gel structures begin to break down, a lower viscosity suspension is produced that is removed and ultimately dissolved in the flowing liquid. A higher shear field from increased pulsation amplitude will cause more gel structure to break down and decrease the viscosity of suspension.

In addition to pressure pulsation, a transient pulsation (water hammer) can cause undesirable pipe stress. Water hammer is currently a concern for the Hanford waste transfer piping, but it is not likely to occur from use of the pressure pulse technology. A stress analysis for selected pipe routes is being performed by WRPS to assess the potential for water hammer. Water hammer is caused by the sudden closure or opening of a valve that significantly changes the velocity of fluid flow in the pipe and results in a "hammering" sound. When a valve in a flowing pipe is suddenly closed, a large pressure spike occurs at the valve. Equation 2 is simplistic but illustrates the maximum pressure differential, $\Delta P$, due to an abrupt change from a flow rate of 6-10 $\mathrm{ft} / \mathrm{sec}$ to zero. As an approximation to determine the magnitude of a pressure surge from water hammer, a supernatant density of $1.3 \mathrm{~g} / \mathrm{cm}^{3}$ and acoustic velocity of seawater at $25^{\circ} \mathrm{C}$, $5,023 \mathrm{ft} / \mathrm{sec}$ (as an equivalent to supernatant waste acoustic velocity) is used. The gravitation acceleration in Equation 2 is $32.2 \mathrm{ft} / \mathrm{sec}$.

$$
\Delta P=\rho a \Delta v / 144 g \quad(\text { Equation 2) }
$$

Where:

$$
\begin{aligned}
& \Delta P=\text { Pressure spike } \\
& \rho=\text { density of the fluid }\left(\mathrm{g} / \mathrm{cm}^{3}\right) \\
& a=\text { Fluid acoustic velocity }(\mathrm{ft} / \mathrm{sec}) \\
& \Delta v=\text { Change in fluid velocity }(\mathrm{ft} / \mathrm{sec}) \\
& g=\text { gravitational acceleration }\left(\mathrm{ft} / \mathrm{sec}^{2}\right)
\end{aligned}
$$

Based on the above values, a water hammer pressure spike for a 2- or 3-in. diameter Schedule 40 stainless steel pipe is calculated and listed in Table 6-1. The results indicate a differential pressure spike equivalent to approximately $530-880 \mathrm{psig}$ (rounded) above a static pressure of 200-300 psig at normal flow rates will form by the effect of water hammer pressure spike. Based on the calculated pressure spike and a static pressure at $300 \mathrm{psig}$, a total pressure surge of approximately 830 to 1,180 psig results from a valve closure and the sudden flow interruption. The Hydrokinetics pulsing would impact the velocity at about $1 \mathrm{ft} / \mathrm{sec}$ (static to a maximum of $20 \mathrm{gal} / \mathrm{min}$ ); approximately an order of magnitude below a water hammer incident. 
Table 6-1. Water Hammer Pressure Surge.

\begin{tabular}{|l|c|c|c|c|c|c|}
\hline & \multicolumn{3}{|c|}{$\begin{array}{c}\text { 2-in. Diameter Schedule 40 } \\
\text { Stainless Steel Pipe }\end{array}$} & \multicolumn{3}{|c|}{$\begin{array}{c}\text { 3-in. Diameter Schedule 40 } \\
\text { Stainless Steel Pipe }\end{array}$} \\
\hline Velocity (ft/sec) & 6 & 10 & 2 & 6 & 10 & 1 \\
\hline Flow rate (gal/min) & 63 & 105 & 20 & 138 & 230 & 20 \\
\hline Pressure spike (psig) & 527 & 879 & 176 & 527 & 879 & 88 \\
\hline
\end{tabular}

\subsection{TRANSPORT AND BLOCKAGE REMOVAL APPROACH}

Hanford waste transfer system operations focus on preventing plug formation, but pipelines may plug and remediation approaches need to be adopted. Standard TFC-ENG-STD-26 describes the nature of various pipeline plugs, prevention measures, and flushing methods to minimize plug or scale formation. The perspective described in this section expands on the TFC-ENG-STD-26 standard. It considers integration of a pulsation technology as an essential component of typical waste transfer system functions. Figure 6-1 is a schematic of the analysis, process decisions, and operational options to consider as wastes of distinct characteristics are transferred in the Hanford waste transfer system.

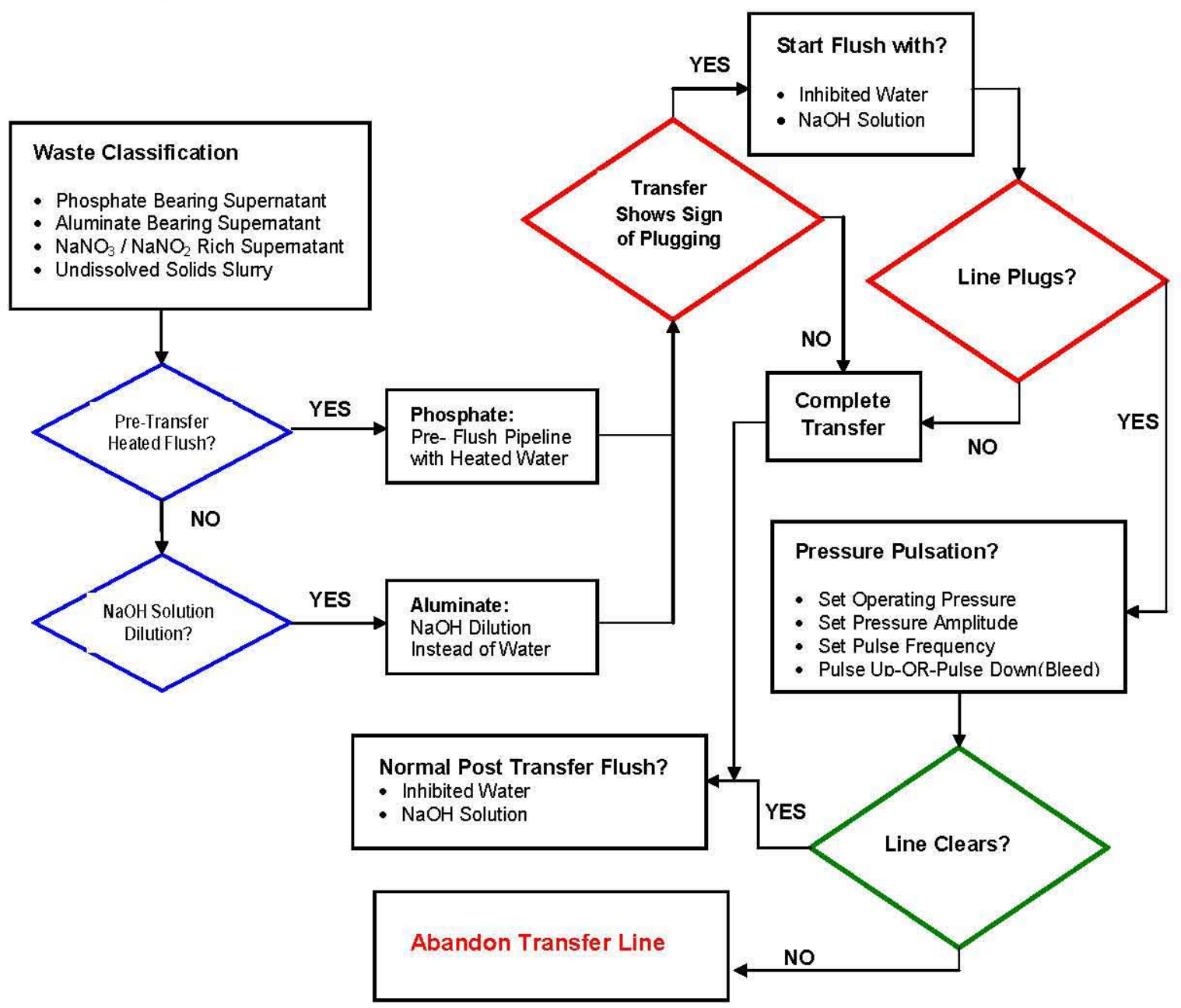

Figure 6-1. Transfer Operation and Pulsation Unplugging Logic 
As illustrated, plugs may be induced by mechanisms of chemical and/or hydrodynamic instability and by settling of solids for the four waste classifications (detailed in Section 4.2) to influence transfer of supernatant wastes or slurry wastes. The chemical composition of a waste needs to be determined at the start of a waste transfer operation to predict foreseeable chemical instabilities related to a waste type. Waste types can be classified as follows:

1. Phosphate-containing supernatant - A supernatant waste containing high concentrations of phosphate with strong temperature dependence to form an interlocking crystalline network and exhibit gelation behavior that plugs the pipeline as it cools down and reaches saturation limits during the transfer.

2. Aluminate-containing supernatant - A supernatant waste containing high concentrations of aluminate ion $\left[\mathrm{Al}(\mathrm{OH})_{4}{ }^{-1}\right]$ may reach saturation limits and precipitate when it is cooled in the pipeline, or when the free hydroxide ion concentration of the solution is decreased by inadequate dilution with water. Precipitation of solids increases the resulting suspension's solids loading and induces hydrodynamic instability when the flow rate reaches the deposition velocity limits and settled solids plug the pipeline.

3. High salt content supernatant - Residual supernatant waste rich in dissolved salts of sodium nitrate and nitrite $\left(\mathrm{NaNO}_{3}\right.$ and $\left.\mathrm{NaNO}_{2}\right)$ remaining in the pipeline may evaporate and adhere to the pipe surfaces. A buildup of hardened solids and hydrated salts may block the next pipeline transfer.

4. Solids containing slurry - The undissolved solids in a slurry waste may settle when the flow velocity is insufficient to keep them suspended, and a moving bed of particles begins to accumulate during a slurry transport operation.

After defining the waste classification, operational decisions need to consider the following:

- Preheating the transfer line when dealing with a phosphate-containing waste to minimize the temperature drop during the transfer

- Diluting a phosphate-containing waste below the saturation limit at a plausible lower temperature during the transfer

- Diluting an aluminate-containing waste with a sodium hydroxide solution instead of water to maintain the supernatant waste hydroxide ion concentration above $\mathrm{Al}(\mathrm{OH})_{3}$ precipitation

- Increasing the pipeline transport velocity to maintain turbulent flow in the slurry transfer line.

A flushing operation is usually initiated when the transfer line shows signs of solids buildup by a gradual change in the line pressure and flow rate. Once again, the options for selecting a compatible flushing solution are considered. For instance, a supernatant rich in phosphate is flushed with water, whereas an aluminate-containing supernatant is flushed with a sodium hydroxide solution. If the line continues to plug, then pipeline pressurization and pulsation can begin immediately without draining the line, which is advantageous in removing the plug. The pulsation parameters of pressure amplitude and frequency, and the maximum and minimum operating static pressure, are adjusted based on a plug characteristic. 


\subsection{ALTERNATIVE 1: HYDROKINETICS}

The Hydrokinetics technology is applied to piping, heat exchanger tubes, and process equipment in plastics, chemical, refining, offshore, and subsea umbilical and flow lines to remove blockage or at a minimum create an aperture in the plug to restore flow in the pipeline. A variety of inorganic and organic foulant buildups (e.g., scale, asphalt, crude oil, cement, polycarbonate, pulp, styrene) have been successfully removed by deploying the technology.

Discussions were held with AIMM Technologies representatives to understand and evaluate the Hydrokinetics technology. ${ }^{9}$ The patent application filings, published literature, and reports on Hydrokinetics technology scoping tests conducted at FIU in 2009 were reviewed. Discussions were held with Dr. Dwayne McDaniel from FIU. ${ }^{10}$ Section 6.3.1 describes the technology, Section 6.3.2 provides examples of Hydrokinetics technology deployment in industry to remove pipeline plugs, and Section 6.3.3 examines FIU testing.

\subsubsection{Technology Description}

The Hydrokinetics patent application, Method and Apparatus for Cleaning Columns by Induced Vibration in Fouling Material and the Column, patent number US 5674323, published in October 1997, and a second patent, Pump Valve Mechanism, patent number US 7421757 , published in September 2008, were reviewed. Both patents are included in Appendix A. Based on the technology description and the operating principles described in Section 6.1, the Hydrokinetics technology uses pressurized fluid pulsation to remove blockage in pipelines. Pressure pulses are created by opening and closing valves. Fluctuations in pressure travel through the pipeline as acoustic waves and form a standing wave in incompressible liquids. Pulsation energy is transferred to the interface between the pipe wall and blockage, and because of the difference in pipe wall and foulant material, they resonate at different frequencies and break the bond between them by induced vibration. Operating pressures for Hydrokinetics action range up to $20,000 \mathrm{psi}$, with a typical range about $250-3,000 \mathrm{psi}$ (patent US 5674323). In this system, the steady-state pressure serves to wash the foulant from the pipe once the bond between the pipe wall and foulant is broken. The Hydrokinetics technology is not a pressuresurge water hammer system common in the pressure-based annular pigging system.

Any source of fluid, including water, sodium hydroxide solution, or other chemical solutions can be used. The system delivers fluid pressure pulses in a pipeline by regulating the pressure differential to transmit positive pressure differentials in the form of positive pressure pulses in the pipeline, or regulated to transmit negative pressure differentials in the form of negative pressure pulses. Positive pressure differentials are transmitted by cyclically opening and closing pneumatic-operated valves controlled manually. The negative pressure differential is managed by removing fluid from the pipeline using a process bleed line or a bypass recycle loop with a dump valve. The valve operation can be automated, but the Hydrokinetics system is operated manually to control interaction between the foulant and the pipe wall by monitoring pressure. Based on discussions with AIMM Technologies representatives, pressure pulses are generated at multiple frequencies of a typical range of 2-30 cycles per minute and up to $1 \mathrm{~Hz}$.

\footnotetext{
9 Contacts: Brooks Bradford Jr., President, and Antone Belcher; website: www.aimmtechnologies.com.

10 Florida International University primary contact point: Dr. Dwayne McDaniel at FIU Applied Research Center; website: www.arc.fiu.edu.
} 
Furthermore, by increasing the pulsation amplitude sufficiently, large oscillations are created between the positive and negative pressure swings in pulse cycles. When liquid pressure falls below its vapor pressure during a negative swing, gas bubbles form and grow. These bubbles are known as acoustic cavitation bubbles. The bubbles are carried in traveling waves. As local pressure reaches above the liquid vapor pressure (positive pressure swing), the bubbles implode and produce a second high intensity and localized pressure wave that enhances pipeline blockage removal. The formation rate, or bulk concentration, of cavitating bubbles depends on the frequency of pulsation when the magnitude of a pulse amplitude reaches acoustic cavitation. The Hydrokinetics technology is aimed at combining the pulsation energy with the resonance of the foulant and the pipe wall at different frequencies, and aimed at adjustment in pulse amplitude to operate above the cavitation threshold. Unplugging is enhanced by the implosion of cavitating bubbles as compared to the pressure pulsation effect alone. Because of the changing nature of pulsation during the Hydrokinetics operation, acoustic cavitation may occur in short time spans with minimum risk of pipeline damage. A typical Hydrokinetics application may last less than 1 hour, and at the most several hours (described by AIMM Technologies representatives).

A schematic process and instrumentation diagram of the Hydrokinetics system is presented in Figure 6-2. As shown, the Hydrokinetics equipment is built around a high-pressure piston pump capable of $20 \mathrm{gal} / \mathrm{min}$ and up to $3,000 \mathrm{psig}$. A pressure relief valve allows solution to recycle at a pressure selected to avoid overstressing the weakest component in the plugged system. The pressure relief of 400-500 psig is for a typical Hanford system deployment, including jumpers. Two air operated valves are used to "pulse" the system up or down through a high-pressure hose with a $1 / 2$-in. to $3 / 4$-in. internal diameter. The down cycle or bleed valve is used only if the plug is firm, and the pressure is cycled up to a maximum several times to "bump" the plug.

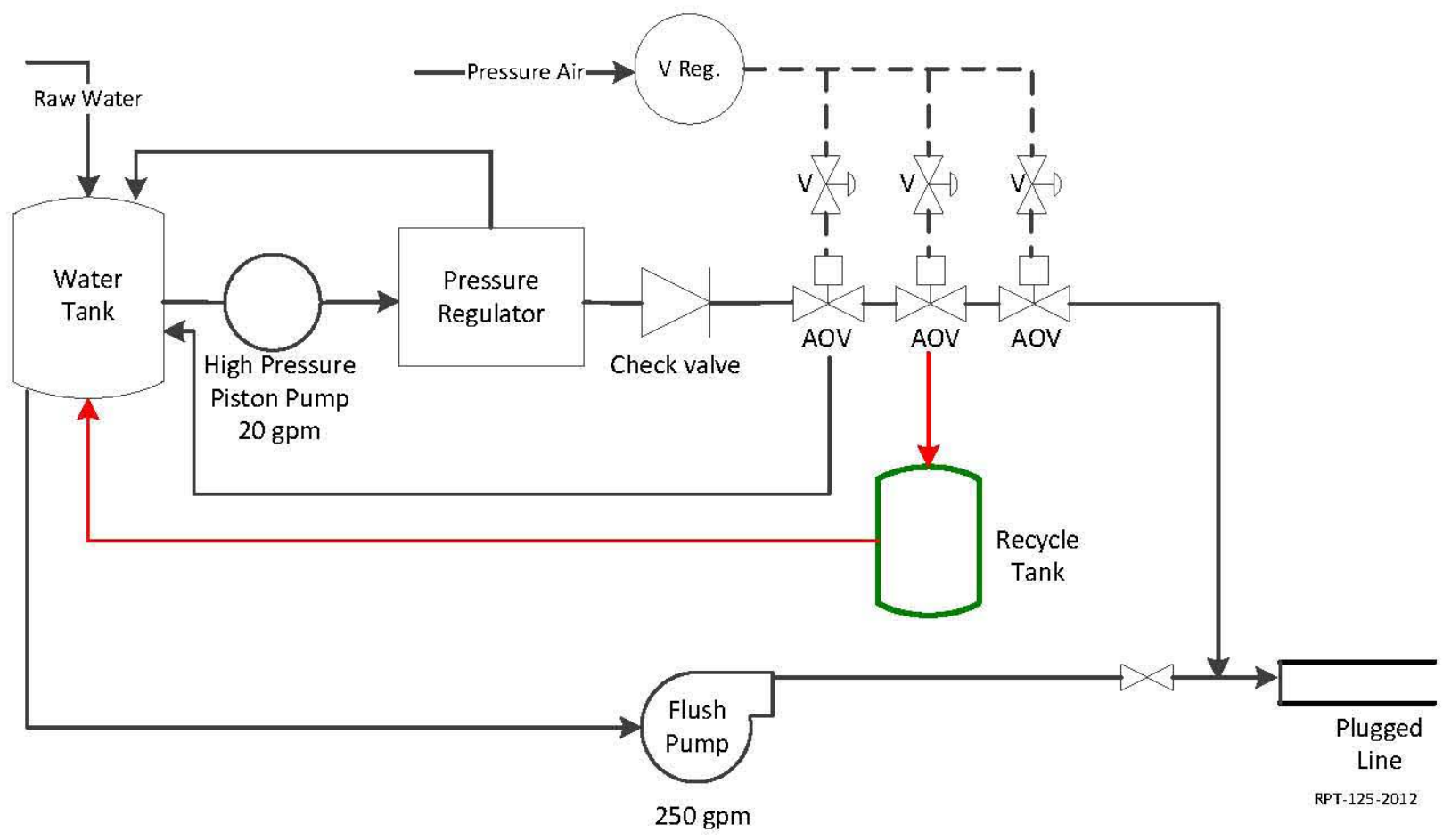

Figure 6-2. Hydrokinetics Process Flow Diagram 
The volume of bleed-back needed to get a pressure drop via the "pulse-down" valve is small because the fluids are nearly incompressible when the line is full. In field operation, backflow is not experienced. Higher pressures are helpful and sometimes necessary; however, the standing wave generated by the valve pulse is the primary reason for the rate of success. To implement the Hydrokinetics system at Hanford and avoid contaminating equipment, the bleed liquid is isolated and routed to a DST or to the transfer line pit drain instead of recirculating within the system. In such a modification, the recycle tank is likely eliminated. Further modification to the Hydrokinetics system is explained in Section 7.0.

The operating strategy is not automated and is enhanced by an experienced operator who learns to see the plug beginning to move by watching the pressure. A typical operating scenario for a maximum operating pressure of $600 \mathrm{psig}$ is listed below and indexed to Figure 6-3:

1. The unplugging effort begins with the lines full, by filling the lines with channel flow bleeding the air out of the system, or by forcing air past the plug at pressure. The goal is to send a standing wave through the water column in the pipe.

2. The operator opens the "pulse-up" valve until the pressure is into a regime that might move the plug (e.g., 400 psig). The valve is open for 30 seconds to several minutes to establish the first pressure goal.

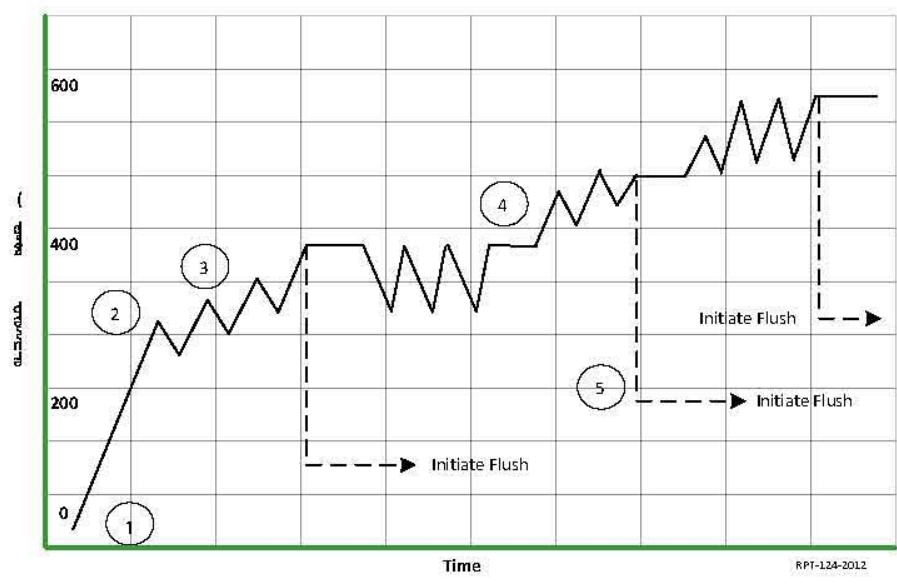

Figure 6-3. Hydrokinetics Unplugging Approach

3. The pulse-up valve is closed and the pressure monitored. The pressure gradually decreases (indicating plug movement or a leaking plug) or remains constant (indicating a hard plug). The pressure normally tails off in a few seconds to a few minutes, and the pulse-up valve is used to bring the pressure back to $400 \mathrm{psig}$. A few of these cycles $(<10)$ typically results in the pressure dropping off at a faster rate, indicating that the plug is breaking or moving.

4. If the pressure does not tail off, the operator uses the pulse-down valve to drop the pressure and bump the plug by alternating up and down cycles about 10 times while monitoring the pressure. If there is no progress, a higher pressure is considered. The maximum pressure would be predefined with a stress analysis for the particular pipe and components involved. After 100 cycles at the maximum pressure over an 8-hour period, a decision is made to abandon the line.

5. Once a plug starts to move, the higher flow rate centrifugal pump is used to flush the plug and line until the flushing rate of $6-10 \mathrm{ft} / \mathrm{sec}$ is established. In the event the plug shows signs of reforming (erratic flow rates), the pulse-up action is repeated while the flush water is on. 


\subsubsection{Existing Field Deployment}

The Hydrokinetics system has been deployed in a variety of pipeline unplugging cases to remove blockage, or at a minimum, to create an aperture in the plug to restore flow in the pipeline. The system has been applied to clean piping tubes, and process equipment in plastics, chemical, refining offshore, and subsea umbilical and flow lines since 1991. It is applied to remove inorganic and organic plugs from process pipelines, heat exchanger tubes, and long-distance flow lines common in the offshore oil explorations.

Analogous to possible pipeline plugs in Hanford Site transfer pipelines, the Hydrokinetics system has successfully handled pipeline blockage or foulants that exhibit pseudo non-creeping and densely packed suspensions like those shown in the photos in Figure 6-4 and Figure 6-5. These extruded plugs maintain varying degrees of structural plasticity. A qualitative view of the rheological character of extruded plugs in Figure 64 indicates that the Hydrokinetics system is able to dislodge concentrated inorganic and organic suspension such as a non-hydrated cement mixture, asphalt, or viscous paraffin that have high yield and tensile stress. This demonstrates an extruded plug shape that is not deformed and maintains a non-creeping nature after being extruded.

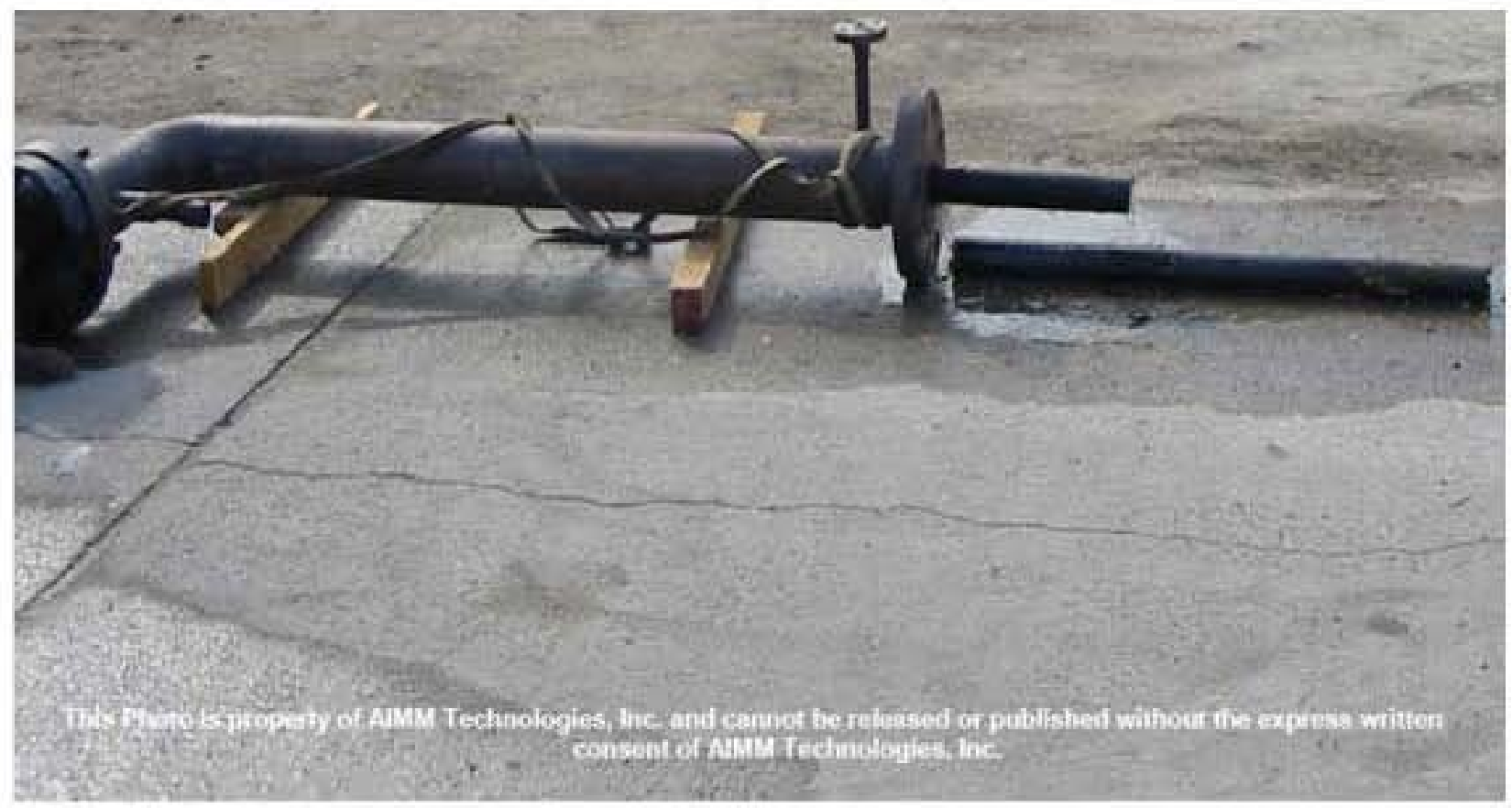

Figure 6-4. High Shear Stress and Viscous Plugs 

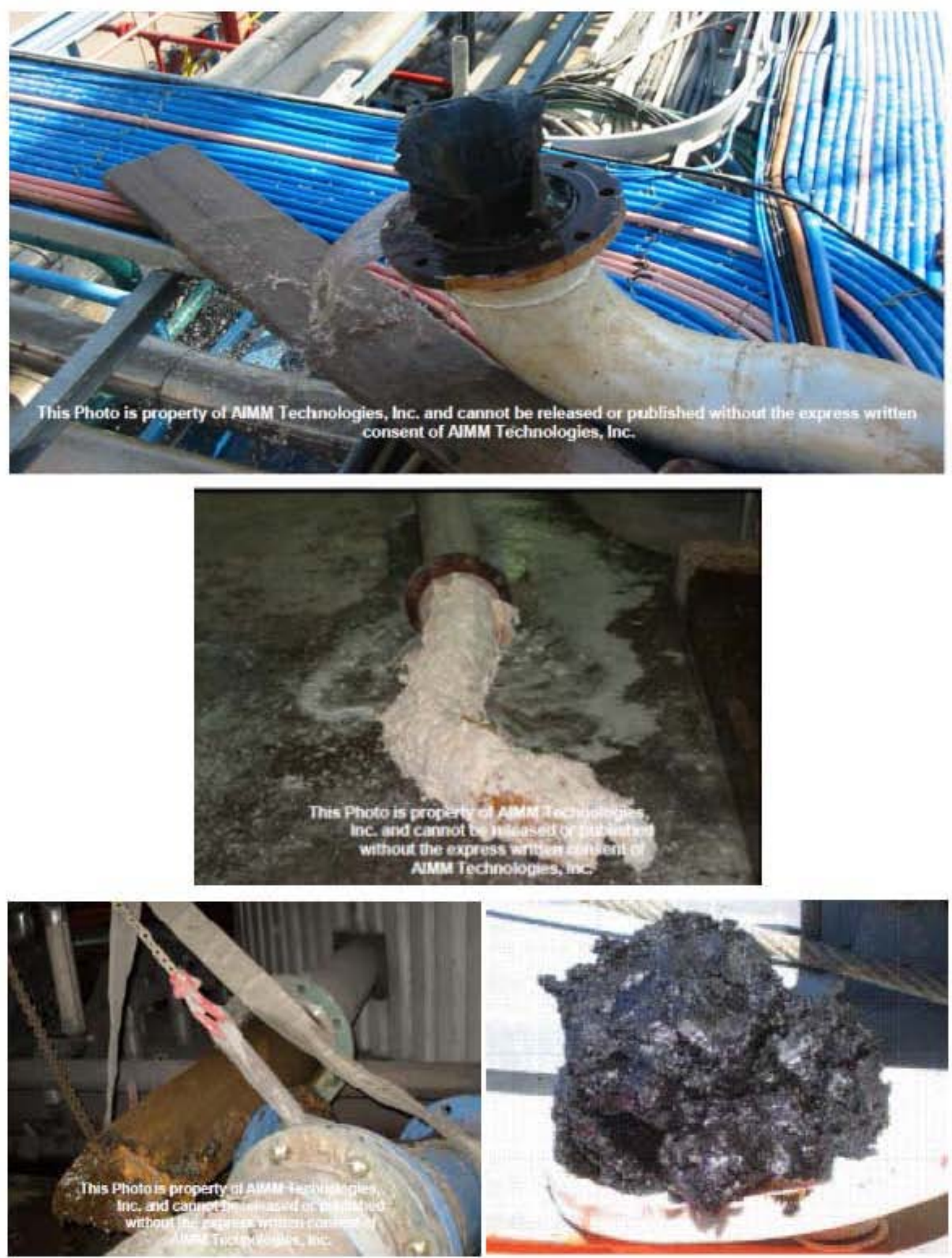

Figure 6-5. Pipeline Blockage or Foulants Handled by the Hydrokinetics Sy stem 
Analogous to a consolidated solid form plug seen in past Hanford pipeline plugs, the Hydrokinetic system has been successfully used to remove a hydrated cement, solidified polyorganic buildup, and heat exchanger tube scale from process pipelines. An example of these types of foulants is shown in Figure 6-6. Additional photos of solidified pipeline plugs removed by the Hydrokinetics system are included in a 2004 Oak Ridge National Laboratory (ORNL) evaluation report, Pipeline Unplugging Assessment and Recommendations for Fermald Environmental Management Project (Morris et al. 2004). The ORNL report provides a list of industry clients using the Hydrokinetics system. A copy of Section 3.5 and Appendix I of the ORNL report is provided as Appendix D.

\subsubsection{Scoping Tests with Pipeline Plug Simulants}

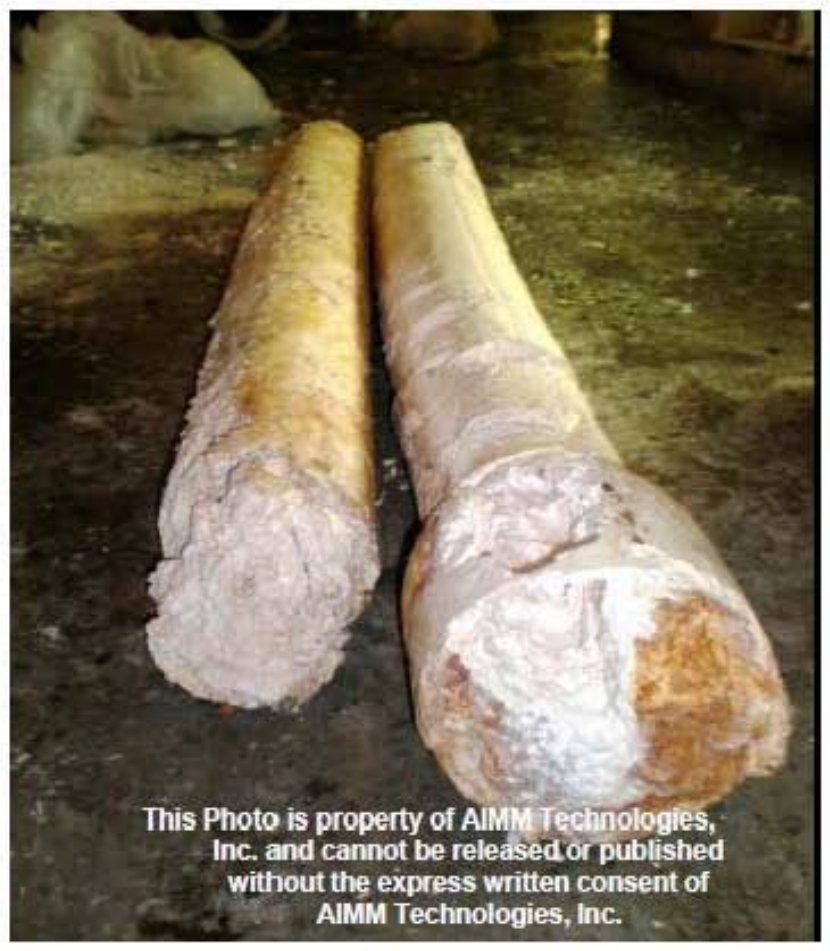

Figure 6-6. Consolidated Form Plug

In 2009, FIU conducted experiments using the Hydrokinetics system to remove pipeline plugs made of a clay-based sludge and two crystallized salt simulants. The results are detailed in a draft topical report (Unplugging of High Level Waste Transfer Pipelines: AMMM Technologies' Fydrokinetic Process Technology Assessment [FIU 2009]), which is included in Appendix B of this document. Excerpts were presented at the 2010 Waste Management Conference (Gokaltun et al. 2010). Three different test pipelines $(310,646$, and $1,822 \mathrm{ft}$ ) constructed from 3 -in. diameter Schedule 10 carbon steel pipe, and 21 - $\mathrm{ft}$ long segments joined by Victaulic ${ }^{11}$ couplings were used to examine the Hydrokinetics system performance in a range of pipeline lengths and configurations with 90-degree elbows (shown in Figure 6-7). Pipeline orientation for the three length settings is illustrated in black, and the location of plug is seen in red and circled in green (see Figure 6-7). A pipeline plug, circled in green, is located before a 90 -degree elbow connected to a $21-\mathrm{ft}$ discharge section. Each plug is placed downstream from an expansion joint configuration that is constructed of thre $10-\mathrm{ft}$ sections intended to simulate the cross-site lines expansion joints (see Figure 3-2). The simulated expansion joint can be removed to examine the Hydrokinetics performance in both configurations. Blockage lengths of $4 \mathrm{ft}$ and $8 \mathrm{ft}$ were used in testing all thre e configurations.

\footnotetext{
${ }^{11}$ Victaulic is a registered trademark of Victaulic Company, Easton, Pennsylvania.
} 
Each pipeline configuration was instrumented with dynamic and static pressure transducers, temperature thermocouples, a $300 \mathrm{psig}$ pressure relief valve, and two 34201A type accelerometers with $\pm 2 \mathrm{~g}$ range connected to a data acquisition system. The accelerometers were installed at the pipeline inlet and on the blockage section to determine the pipe wall vibration profile between the inlet and the blockage in various configurations. The dynamic pressure transducers monitored pressure fluctuations not recorded by static pressure transducers.

As shown in Figure 6-7, simulated plugs were placed before a final 90-degree elbow connected to a discharge section. After conducting introductory tests with simulants to determine minimum extrusion pressure for a blockage type, a bentonite clay and water sludge at $68 \mathrm{wt} \%$ bentonite clay, a hard saltcake simulant of hydrated potassium-magnesium sulfate (K-Mag), and a crystallized sodium aluminosilicate (Na-Al-Si or NAS) were selected for testing. The selection was based on using simulants to exceed an extrusion pressure of $150 \mathrm{psig}$ at a $4-\mathrm{ft}$ blockage length. The extrusion pressure limit originated from the lower limit criterion of the hydrokinetic system operating at 100 psig.
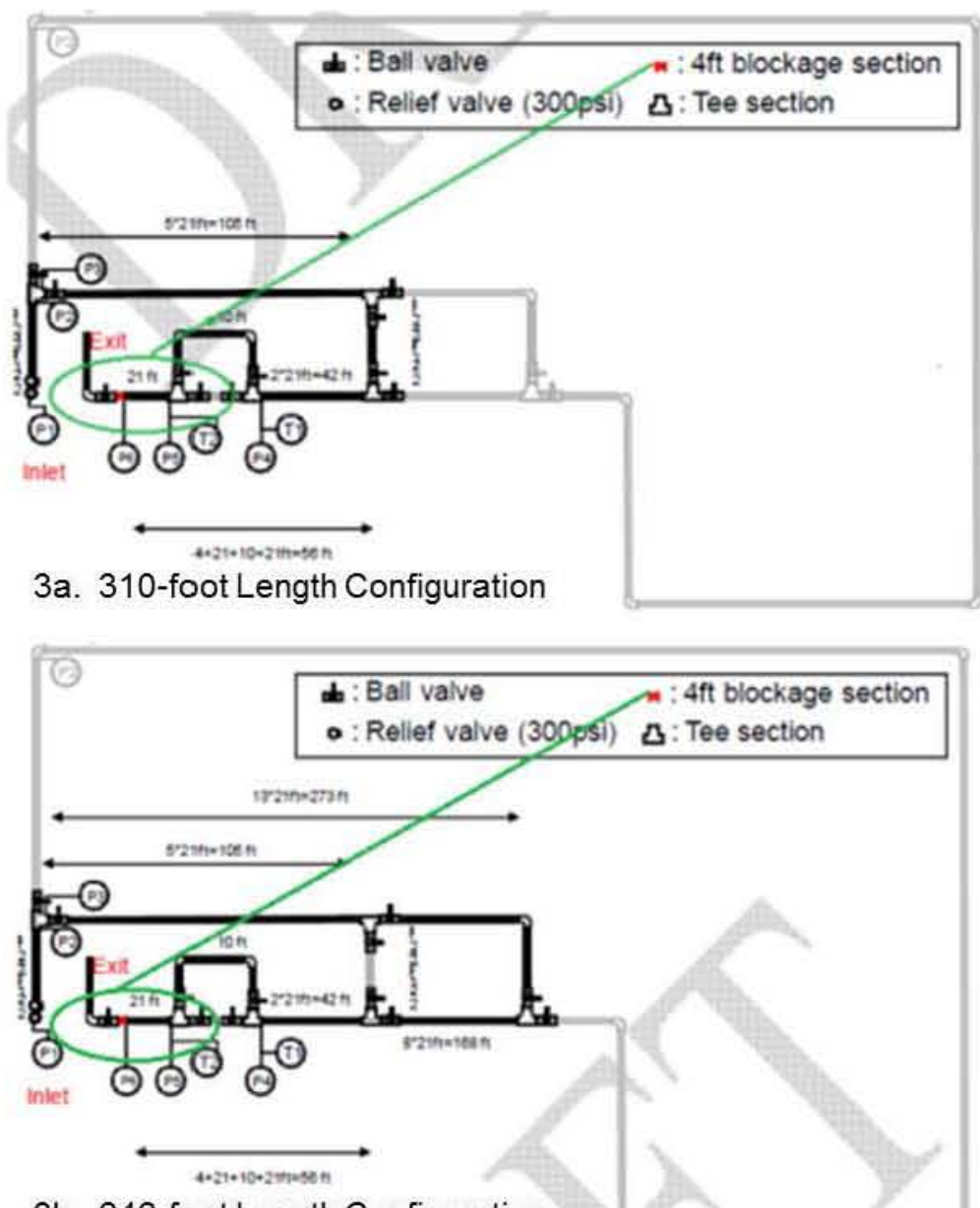

3b. 646-foot Length Configuration

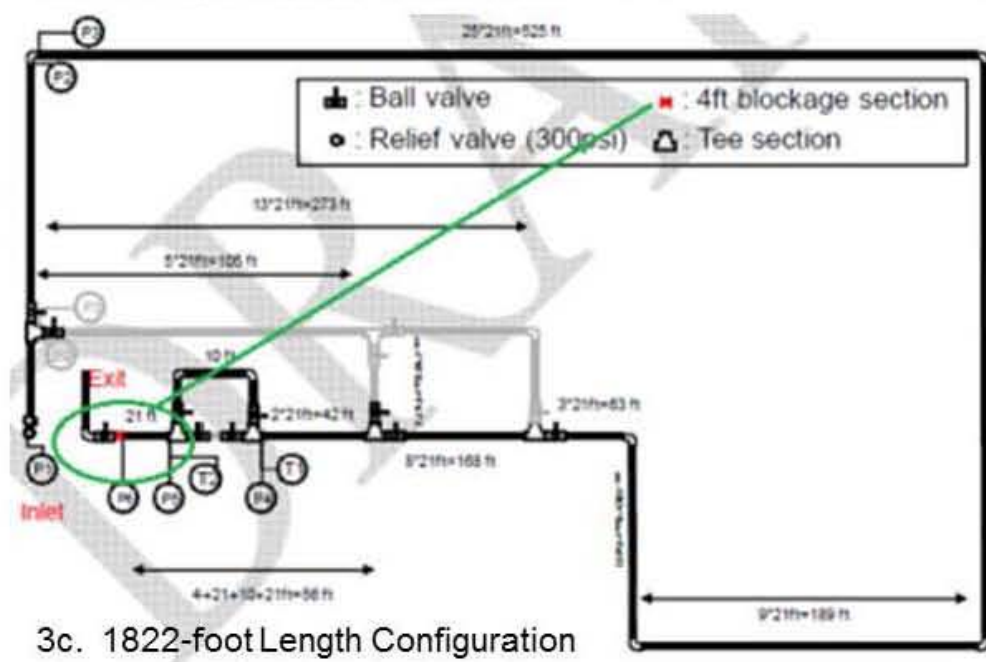

Source: FIU, 2009, Unplugging of High Level Waste Transfer Pipelines: AIMM Technologies' Hydrokinetic Process Technology Assessment, Florida International University, Miami, Florida.

Figure 6-7. Illustrations of Test Pipeline Length Configurations 
Extrusion tests presented in Table 1 of FIU (2009) showed the following:

- 4-ft $68 \mathrm{wt} \%$ bentonite clay in water plug required approximately $100-170$ psig pressure

- 4-ft K-Mag hard saltcake, at a composition of $90 \mathrm{wt} \%$ powder mixed with $10 \mathrm{wt} \%$ water, required above 600 psig pressure

- 4-ft NAS crystallized salt plug required approximately 500 psig pressure.

To increase extrusion pressures, the length of plugs for the bentonite clay sludge ( $68 \mathrm{wt} \%$-solids) was increased to $8 \mathrm{ft}$ to reach above a 190 psig extrusion pressure. Ultimately, in testing the Hydrokinetics system, bentonite/water sludge plugs of 8- and 12-ft lengths were used. The Hydrokinetics system tests are summarized in Table 9 of FIU (2009) and presented in Table 6-2. Besides the results shown in Table 6-2, three tests with 2-ft length K-Mag plugs placed in a $310-\mathrm{ft}$ length configuration were performed. The 2 - $\mathrm{ft}$ K-Mag plugs were partially unplugged at $60 \mathrm{psi}, 100 \mathrm{psi}$, and $280 \mathrm{psi}$ maximum pressures. The results showed the Hydrokinetics system was successful in removing bentonite clay simulant plugs and demonstrated limited success in removing crystallized salt plugs. At times, the system showed an ability to create an aperture in the plug to restore flow in the K-Mag simulant.

Table 6-2. Hydrokinetics System Unplugging Tests at Florida International University.

\begin{tabular}{|c|c|c|c|c|c|c|c|}
\hline \multicolumn{8}{|c|}{ Pipeline Unplugging Tests } \\
\hline Test \# & $\begin{array}{c}\text { Expansion } \\
\text { joint }\end{array}$ & $\begin{array}{l}\text { Distance to } \\
\text { plug (ft) }\end{array}$ & $\begin{array}{l}\text { Simulant } \\
\text { plug }\end{array}$ & $\begin{array}{l}\text { Plug length } \\
\text { (ft) }\end{array}$ & $\begin{array}{c}\text { Pressure } \\
\text { (psig) }\end{array}$ & $\begin{array}{l}\text { Unplugging } \\
\text { success }\end{array}$ & $\begin{array}{l}\text { Duration } \\
\text { (min) }\end{array}$ \\
\hline 1 & 1 & 310 & Bentonite $^{a}$ & 8 & 272.2 & Yes & 16 \\
\hline 2 & 1 & 310 & Bentonite $^{\mathrm{a}}$ & 12 & 286.3 & Yes & 30 \\
\hline 3 & 1 & 310 & K-Mag ${ }^{\mathrm{b}}$ & 4 & 265.1 & Partial & 40 \\
\hline 4 & 1 & 310 & $\mathrm{Na}-\mathrm{Si}-\mathrm{Al}^{\mathrm{c}}$ & 4 & 292.8 & No & 52 \\
\hline 5 & 0 & 310 & $\mathrm{Na}-\mathrm{Si}-\mathrm{Al}^{\mathrm{c}}$ & 4 & 285 & No & 21 \\
\hline 6 & 1 & 646 & Bentonite $^{a}$ & 8 & 225.3 & Yes & 17 \\
\hline 7 & 1 & 646 & Bentonite $^{\mathrm{a}}$ & 12 & 264.2 & Yes & 18 \\
\hline 8 & 1 & 646 & K-Mag ${ }^{\mathrm{b}}$ & 4 & 287.1 & No & 54 \\
\hline 9 & 1 & 646 & $\mathrm{Na}-\mathrm{Si}-\mathrm{Al}^{\mathrm{c}}$ & 4 & 278.3 & No & 41 \\
\hline 10 & 1 & 1822 & Bentonite $^{\mathrm{a}}$ & 8 & 291.7 & No & 115 \\
\hline 11 & 1 & 1822 & Bentonite $^{a}$ & 12 & 214.2 & Yes & 73 \\
\hline 12 & 1 & 1822 & K-Mag ${ }^{\mathrm{b}}$ & 4 & 318.7 & No & 40 \\
\hline 13 & 1 & 1822 & $\mathrm{Na}-\mathrm{Si}-\mathrm{Al}^{\mathrm{c}}$ & 4 & 268.8 & No & 52 \\
\hline
\end{tabular}

Source: FIU, 2009, Unplugging of High Level Waste Transfer Pipelines: AIMM Technologies' Hydrokinetic Process Technology Assessment, Florida International University, Miami, Florida.

${ }^{a}$ A bentonite clay and water mixture containing $68 \mathrm{wt} \%$ bentonite clay was used.

${ }^{\mathrm{b}}$ A hydrated potassium-magnesium sulfate (K-Mag) saltcake from mixing grounded potassium-magnesium sulfate granular crystal added to $10 \mathrm{wt} \%$ water was used.

c A modified formulation of sodium aluminosilicate (NAS) simulant originally described in PNNL-17758, Preparation and Characterization of Chemical Plugs Based on selected Hanford Simulants, was used. 
All tests were conducted when the pipelines were drained, as documented in FIU (2009) (see Appendix B), and the Hydrokinetics system was operated without vacuuming or attempting to remove air pockets from the pipeline. Limited attention to the presence of air in the pipeline drastically reduced the effectiveness of pressure pulses traveling as a standing acoustic wave of pressure pulse (discussed in Section 6.1). It was reported that the pressure pulses attenuated significantly from the inlet to the blockage section during the unplugging process as a result of the air presence. It is plausible that the presence of air pockets in the pipeline prevented transmission of the pressure pulses to the plug face. Therefore, this evaluation concluded that the FIU testing was a reasonable initial scoping attempt to investigate the Hydrokinetics system capability at low operating pressures of up to $300 \mathrm{psig}$. However, the presence of the air pocket and significance attenuation of pressure pulses will need to be factored in the conclusions about the Hydrokinetics system's limited ability to remove the crystallized salts of K-Mag and $\mathrm{Na}-\mathrm{Al}-\mathrm{Si}$, which complicates the assessment of the Hydrokinetics system for use in unplugging pipelines at Hanford.

Evaluation of the Hydrokinetics system was further limited by the range of simulants selected to emulate Hanford pipeline plugs. The testing used a bentonite clay and water sludge at $68 \mathrm{wt} \%$ solids that undergoes osmotic swelling when exposed to water and exhibits highly elastic characteristics following a Bingham-plastic behavior (PNNL-11103, Retrieval Process Development and Enhancement Project Fiscal Year 1995 Simulant Development Technology Task Progress Report). A bentonite clay sludge shear strength increases linearly with solids loading (see Figure 2.9 of PNNL-11685, Retrieval Process Development and Enhancements Waste Simulant Compositions and Defensibility) and tends to adhere less to surfaces when compared to a kaolin clay sludge. A kaolin clay sludge rheology is dominated by viscous characteristics, compared to a bentonite clay sludge at similar solids loading, and tends to adhere to surfaces more readily. Based on these observations, a kaolin clay sludge at similar shear strength represents a sludge plug of increased adhesion to the pipeline surface compared to a bentonite clay sludge.

In contrast, a closer examination of the crystallized salt simulants used in the Hydrokinetics system testing reveals an excessively conservative K-Mag crystallized salt simulant, and a NAS simulant that crystallizes in time and exhibits changing characteristics. These simulant choices may inadequately represent the Hydrokinetics system performance against the nature of plugs in the Hanford waste transfer lines. As described in PNNL-11103 and PNNL-11685, and referenced in the FIU testing for K-Mag selection, the original development of a granular potassium-magnesium sulfate mixed with varying water concentration was intended to mimic hydrated hard simulant monoliths of approximately $10-30 \mathrm{MPa}$ (approximately 1,400-4,000 psi) compressive strengths for high-pressure waterjet-based retrieval applications. At the time (PNNL-11103 and PNNL-11685), the K-Mag saltcake simulants were designed to provide a conservative challenge to examine the high-pressure waterjet systems used in retrieving hard saltcake and hardpans from the bottom of storages tanks, with varying mechanical strengths and porosities (see Figures 2.17 and 2.18 of PNNL-11685). The mechanical properties of tank saltcakes or crystallized salts formed in the pipeline have not been measured, and using high mechanical strength K-Mag simulants in the pipeline unplugging tests may overestimate the actual characteristics of hardened hydrated salt plugs in pipelines induced by evaporation of supernatants rich in sodium nitrate and nitrite solutes. 
In the FIU testing, the K-Mag formulation (at $90 \mathrm{wt} \% \mathrm{~K}$-Mag) was altered by grinding the starting granular potassium-magnesium sulfate into powder before mixing with water. The change in K-Mag particle size distribution produced a K-Mag monolith of lower porosity and elevated mechanical strength, and adversely increased conservatism in the simulant characteristics.

A review of the original NAS crystallized salt formulation was based on developing pipeline chemical plugs to exhibit amorphous gelation characteristics (PNNL-17758, Preparation and Characterization of Chemical Plugs Based on Selected Hanford Simulants). Reported analysis of the sodium aluminosilicate (NAS) gel indicated the growth of sodium nitrate crystals as a function of time, which altered the plug nature and produced a chemically unstable simulant formulation. Extrusion test results showed the NAS simulant mechanical strength changes within the length of the NAS specimen. The strength of plug segments extruded from the bottom of the pipe was greater than the sections in the upper and middle part of the pipe (PNNL-17758). It is plausible that the changing nature of NAS characteristics during the Hydrokinetics system testing resulted in an undesirable variability that impacted the evaluation outcome.

Based on limitations in the FIU testing, a comprehensive test using improved simulants to better emulate the four classes of plugs formed in the Hanford waste transfer system may provide significant insight in evaluating the Hydrokinetics system.

\subsubsection{Deployment Safety Aspects}

The current classification of the Hanford waste transfer lines is Hazard Category 2 safety significant (RPP-13033, Tank Farms Documented Safety Analysis). The documented safety analysis (DSA) establishes the safety basis for the tank farms nuclear facilities and documents the results of hazard and accident analyses for the tank farms facilities and their operation. The DSA also describes the significant features and programs that prevent or mitigate the identified nuclear hazards and establishes the envelope within which the facilities can continue to safely operate. Hazards analyses are performed by procedure TFC-ENG-DESIGN-C-47, Process Hazard Analysis.

The installation of either a portable or permanent pulse system poses similar risks compared to current tank farms operations. The portable pressure pulse system is functionally similar to current hydrostatic testing, and a permanent pressure pulse system is similar to the existing flushing design concept planned for the AY/AZ Farm. Either approach requires that hazards be addressed in the context of the DSA. Key areas typically monitored to assure safe operation for an unplugging system are described below.

The primary waste transfer line is safety significant, and the pressure pulse system used for pipeline unplugging is an interface with the primary transfer line. The boundary for defining which components are safety significant is determined by safety reviews and hazards analysis, as described in RPP-RPT-42297, Safety-Significant Waste Transfer Primary Piping Systems Functions and Requirements Evaluation Document. Safety-significant components are identified as the details of the piping and instrumentation diagram (P\&ID) are defined. The valves, pressure relief devices, and control systems required to maintain a confinement boundary under every potential process or accident scenario will be safety significant. 
In the event of a plug, the waste transfer pump would be administratively locked out such that the pressure pulsing and flushing operations could not cause a waste transfer leak accident. The pulsing/flushing equipment is an interfacing water system, and defense-in-depth features would apply (see RPP-13033, Section 3.3.2.3.2). Under this scenario, the unplugging equipment may not include safety-significant components.

Overpressure - A stress analysis is required for all transfers in the DST system (TFC-MD-085, Design Conditions for Analyses of Process Piping). The final approach recommended for a pressure pulse system will undergo the same rigor for stress analysis to verify the safety basis limits are not exceeded. The analysis specifically addresses the dynamic effects of hydraulic shock, including water hammer, surge, and column separation. The components of the pressure pulse system are reviewed with a failure modes and effects analysis to determine if any failure scenario exceeds the pipe stress ceilings defined in TFC-MD-085, which defines the safety basis. The components of the design required to ensure compliance with the safety basis are designated safety significant (e.g., pressure relief valves).

For analyses that determine the potential overpressure conditions resulting from waste transfer pump operations or the dynamic effects of hydraulic shock cannot exceed specified safety basis failure limits, pressure-relieving devices (if present) shall not be credited with limiting pressure in piping, unless they are qualified as safety-significant under the procedural controls governing tank farms administration, engineering, and operations (TFC-MD-085, Section 3.2 (2.b)).

Spray leak - Components pressurized above 400 psig during pipeline unplugging activities that use higher than normal operating pressures are at risk of leaking. PUREX connectors have gaskets designed for 400 psig that could result in a spray leak. A pit cover block or portable cover system would be used to contain a spray leak. However, the valve pits are not ventilated with a filtered confinement system, so the potential exists for a contamination spread following a spray leak.

Backflow - The cross-site transfer line slopes up to a mid-point such that drainback occurs if the jumpers or valve are not properly configured. During an unplugging activity, the air volume between the plug and the nozzle are compressed and provide a motive force for backflow. Proper routings to drain the line and a means to protect against backflow to clean systems must be provided.

Misrouting - Pits typically have multiple wall nozzles and multiple valves located in the jumpers. Misrouting from the wrong wall connection or a wrong valve setting must be overcome with administrative controls.

Pulse valve recycle - The Hydrokinetic pressure pulse valve configuration recycles solution during the depressurization or pulse-down phase. For contaminated applications at Hanford, only fresh raw water or inhibited water are introduced into the unplugging equipment. The pulse-down phase is abandoned and the transfer line drained with a valve to the valve pit floor drain or to another pipe route via a wall nozzle. The routing from the pressure pulse equipment is protected with backflow prevention. 


\subsubsection{Regulatory Impacts}

Environmental regulations from applicable federal and Washington State sources will be reviewed and adhered to. The Washington State Department of Ecology (Ecology) is in the process of issuing a Final Status Permit for the DST system that will include conditions for the operation of all transfer lines. The final version of the permit, when issued (anticipated by Ecology to be spring of 2013), will need to be reviewed to see if changes to current procedures will be affected.

\subsubsection{National Environmental Policy Act}

Overview - The National Environmental Policy Act of 1969 (NEPA) is the legal driver to prepare an environmental impact statement (EIS). Federal law requires the completion of an EIS

"[for] major Federal actions significantly affecting the quality of the human environment." An EIS must address:

... (i) the environmental impact of the proposed action, (ii) any adverse environmental effects which cannot be avoided should the proposal be implemented, (iii) alternatives to the proposed action, (iv) the relationship between local short-term uses of man's environment and the maintenance and enhancement of long-term productivity, (v) and any irreversible and irretrievable commitments of resources which would be involved in the proposed action should it be implemented.

Transfer Lines NEPA coverage - The use of transfer lines within the DST system and to provide waste feed to the WTP is addressed in existing NEPA documentation under DOE/EIS-0189, Tank Waste Remediation System, Hanford Site, Richland, Washington, Final Environmental Impact Statement. The information is further updated by the recently released DOE/EIS-0391, Final Tank Closure and Waste Management Environmental Impact Statement for the Hanford Site, Richland, Washington (TC \& WM EIS). The implementation of a pipeline unplugging technology at the Hanford tank farms will be reviewed for NEPA implications, but will likely fall within the scope of these existing NEPA documents. A NEPA checklist will be developed once a final technology is down-selected in accordance with WRPS procedures.

\subsubsection{Washington State Requirements}

The liquid and solid mixed waste (i.e., radioactive and hazardous) in the Hanford underground storage tanks includes hazardous wastes that are subject to the Resource Conservation and Recovery Act of 1976 (RCRA). RCRA regulates hazardous waste from the point of generation through storage, treatment, and ultimate disposal. Many aspects of Hanford Site operations are governed by various RCRA permits. The U.S. Environmental Protection Agency (EPA) RCRA implementing regulations also include minimum technological requirements (e.g., secondary containment) that apply to management of hazardous wastes. The state of Washington, via Ecology, has been authorized by EPA to administer a state hazardous waste management program to implement a federal hazardous waste management program, as set forth in the Revised Code of Washington (RCW) 70.105, "Hazardous Waste Management Act of 1976," through Washington Administrative Code (WAC) 173-303, "Dangerous Waste Regulations." 
Unplugging Implications - The design and construction of the transfer lines was reviewed by Ecology, who required submittal of a stamped report prepared by an independent qualified registered profession engineer (IQRPE). Recovery from a waste transfer line plug may require a review of the approach and verifying the fitness-for-service review, depending on the pressures used to clear the line. A waste transfer line plug may be treated similar to a transfer line failure and repair, and Ecology could require a review of fitness-for-service by an IQRPE in accordance with the following Washington State code:

If the owner/operator has repaired a tank system in accordance with (e) of this subsection, and the repair has been extensive (e.g., installation of an internal liner; repair of a ruptured primary containment or secondary containment vessel), the tank system must not be returned to service unless the owner/operator has obtained a certification by an independent, qualified, registered, professional engineer in accordance with WAC 173-303-810 (13)(a) that the repaired system is capable of handling dangerous wastes without release for the intended life of the system. This certification must be submitted to the department within seven days after returning the tank system to use. (WAC 173-303-640 7(f), "Certification of major repairs")

In addition, any contaminated equipment, debris, and materials resulting from the unplugging exercise will need to be handled and disposed in a compliant manner, in accordance with the requirements of the permit and WAC 173-303.

\subsubsection{Risk Analyses}

The following are examples of project and technical risks that must be addressed to implement a pressure pulse system for unplugging waste transfer lines in the tank farms.

Overpressurization of piping - The standard pump provided by the vendor for waste transfer line unplugging activities can exceed the maximum pressure rating for the piping. The issues can be addressed administratively by controlling the pump pressure and passively with pressure relief valves. It is also possible to automate pump shutdown and designated pressures via the control system. This risk is mitigated with multiple layers of protection, both administrative and passive. The exact approach will be optimized as a part of the detailed design and safety review process.

Pipe stress from pressure pulse action - The potential exists during waste transfer line pulsing to induce a standing wave that coincides with a natural harmonic in the piping that serves to propagate or magnify the wave and create a water hammer affect. In discussions with vendors, there has been no evidence of water hammer or vibration in the 2-30 cycles per minute frequency range.

A stress analysis is required for all transfers in the DST system (TFC-MD-085). The final approach recommended for a pressure pulse system will undergo the same rigor for stress analysis to verify the safety basis limits are not exceeded. This assessment for compliance with the safety basis is now a requirement for the justification for continued operation (discussed further in Section 7.2.1). 
ALARA principles - The dose to workers is a function of the contamination levels in the valve pits. If a PUREX connector or another remote connecting device is used, manual entry into the valve pit can be avoided. However, if the pipe nozzle requires a manual or welded connection, the pit will need to be decontaminated before personnel entry. Doses for pit entry are higher and activities are monitored from an ALARA standpoint to minimize personnel exposure.

Valve pit entries are avoided if possible, but are occasionally necessary at Hanford and can be done safely.

Project execution - The implementation of an unplugging strategy has important project schedule implications for recovering a plugged waste transfer line. The likely scenario is a portable pressure pulse system that is ready for use prior to a plugging event. The implementation of a portable pressure pulse system resembles hydrostatic testing, and with procedures in place, the equipment can be deployed and waste transfer line unplugging can begin in a few days to a few weeks. If the procedures are not in place, a work package is developed and one to three months are likely required before work can commence.

The means of connecting the pressure pulse system to the pit nozzle for pressures beyond 400 psig could be the critical path activity. A process for upgrading the present PUREX connector or another connecting strategy should be pre-identified and developed for use to mitigate the schedule impacts.

\subsection{ALTERNATIVE 2: PIPE-PULSE}

The Pipe-Pulse system is a pressure pulsation unplugging system provided by Paradigm Flow Services. Paradigm Flow Services, established in 2010, focuses on technologies to remediate subsea and topside blockage in pipelines and umbilical flow lines for the offshore oil and gas industry. The company is located near Kintore in Aberdeenshire, United Kingdom. In addition to the Pipe-Pulse system, the company uses Flexi-coil, an extended length plumber's snake coiled tubing, to remove blockage from subsea flow lines. The evaluation of the Pipe-Pulse system is based on the patent application filing and publicly available literature. Direct technical discussions and communication with company representatives were not possible due to the Paradigm Flow Services requirement that a stringent nondisclosure agreement be signed.

\subsubsection{Technology Description}

In evaluating the Pipe-Pulse system, a March 2012 patent application (WO 2011/036502 A1, Improved Blockage Removal Apparatus and Method) ${ }^{12}$ was reviewed. This patent application is included in Appendix E. Additional reviews included an article entitled "Pipe-Pulse technology for Subsea Blockage Removal" (Bain 2012) and marketing brochures.

${ }^{12}$ The initial Pipe-Pulse System patent application is filed with World Intellectual Property Organization (WO) and can be searched using the European Patent Register at: www.epo.org/searching/free/register.html. A U.S. patent application for the Pipe-Pulse system was filed in July 2012 with the U.S. patent and trademark office (US 2012/0186661 A1, Blockage Removal Apparatus and Method). 
Similar to the Hydrokinetics system, the Pipe-Pulse system uses pressurized fluid pulsation, referred to as a flow oscillation method in the Bain (2012) article, to remediate blockage in pipelines. The technology is intended to remove blockage in subsea pipelines and umbilical flow lines in the hydrocarbon exploration and production industry where the buildup of wax, paraffin, scale, asphaltene, or viscous hydrocarbons restrict flow lines. The application of the system in distances of 30 miles and pressures up to $10.000 \mathrm{psi}$ is reported. In contrast to the Hanford waste transfer line pressure ratings, the subsea flow lines are typically rated up to 15,000 psi pressures.

Based on the invention description in Patent Application WO 2011/036502 A1, the Pipe-Pulse system delivers fluid pressure pulses with controlled pulsation magnitude by operating pulsation below the cavitation threshold. The system uses cyclically opening and closing valves to deliver a fluid pressure pulse in the pipeline. The pressure differential is regulated to transmit positive pressure differentials in the form of pulses (pulse amplitude), or regulated to transmit negative pressure differentials in the form of negative pressure pulses. The negative pressure differential is managed by removing fluid from the pipeline using a process bleed line or a bypass recycle loop with controllable valves. In this configuration, both positive and negative pressure pulses are transmitted to the pipeline. For example, a series of positive pressure pulses in a pressure-up cycle, followed by a series of negative pressure pulses during the pressure bleeding cycle, are transmitted in the pipeline. The system uses oscillating valves to reach frequencies up to $10 \mathrm{~Hz}$.

A schematic process and instrumentation diagram of the Pipe-Pulse taken from the pipe pulse patent (see Appendix E, page E-22) is presented in Figure 6-8. As shown, the Pipe-Pulse system apparatus (11) is connected to the fluid pipeline (32) with an isolation valve. The Pipe-Pulse apparatus is also connected to a high-pressure pump (14) to transfer fluid from a liquid vessel (12), and is regulated by an automated two-way pressure regulator interfaced with a computer to control regulator output pressure. The pressurized fluid is transferred to a pressure accumulator to provide a uniform pressure input to the first oscillating valve and thereby minimize the variation in pulse amplitude output of the oscillating valve while pulsating. A one-way solenoid actuated valve (26) capable of opening and closing at up to $10 \mathrm{~Hz}$ is used in the higher pressure (positive differential pressure) line. Well-defined fluid pulses are transferred to the conduit, and pulse occurrence is measured by a pressure sensor (82) interfaced with an external module (i.e., computer). A second one-way solenoid actuated valve (36) is placed in a fluid return line from the conduit. The second valve actuates during the pressure bleed cycle (negative differential pressure) to recycle fluid to the pressure regulator liquid vessel. A controllable dump valve (40) is installed between the solenoid actuated valve (36) and the pressure regulator. 


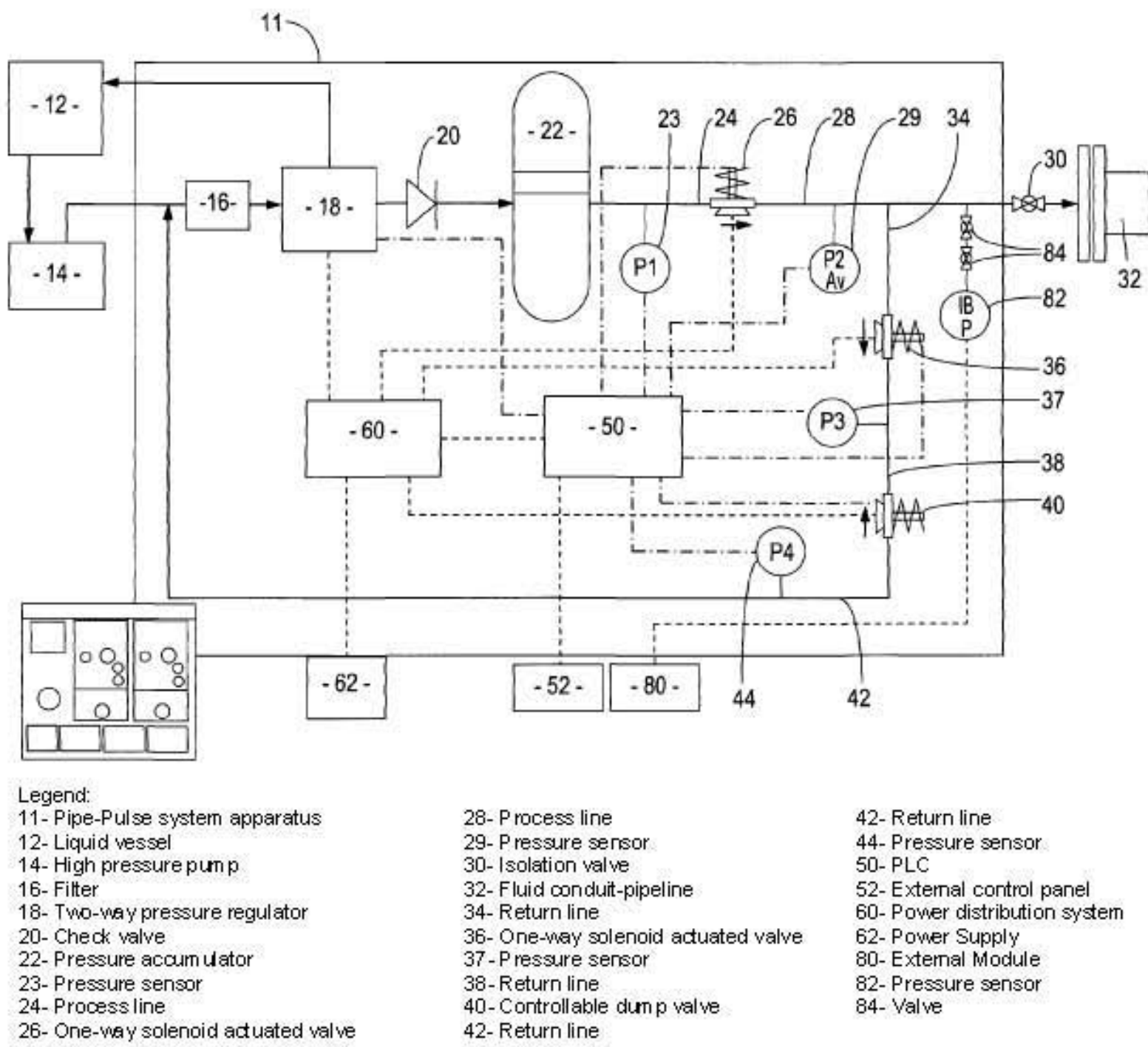

Figure 6-8. Pipe-Pulse System Process and Instrumentation

A programmable logic controller (PLC) unit communicates with the two solenoid actuating valves, the controllable dump valve, and pressure regulator. The PLC unit is interfaced with an external control panel (52) for user operation. The external control panel is set to control the operating frequencies of the two oscillation valves, the pressure differential, and the maximum and minimum pressure. The control panel is supplied with an on/off switch and a pressure regulator override function, and a visual indicator for status of the various Pipe-Pulse system components. Power is supplied by an external power supply and distributed to various components by a power distribution system (60). Pressure sensors measure the pressure output at various locations, as depicted in Figure $6-8$, and provide pressure measurement signals to the PLC unit.

A typical operation of the Pipe-Pulse system is described in Patent Application WO 2011/036502 A. 1 and starts with connecting the apparatus to the conduit and opening the isolation valve. The two oscillating valves in the forward and return lines remain closed, the dump valve remains in open position, and the regulator is fully opened. The parameters of frequency for valve (26), pulse amplitude (maximum differential pressure), and the steady-state pressure maximum and minimum range are selected and entered on the external control panel. 
The pump is activated while valve 26 remains closed, and a baseline pressure is measured. The controllable regulator pressure is increased to a selected value of steady-state pressure, $P_{S S}$, and differential pressure for the pulse amplitude, $P_{\text {cyclic }},\left(P_{S S}+P_{\text {cyclic }}\right)$. Then the valve oscillation is started based on a PLC command at preset oscillation frequency to transmit positive pressure pulses in the conduit to remove the blockage. The two-way regulator is automatically adjusted by the PLC to maintain $\left(P_{S S}+P_{\text {cyclic }}\right)$ during pulsation. The oscillating valve (26) automatically closes when measured pressure (P1 in Figure 6-8) exceeds the preset $\left(P_{S S}+\right.$ $P_{\text {cyclic }}$ ) limits.

A PLC communicates with the two solenoid actuating valves, the controllable dump valve, and pressure regulator. The PLC is interfaced with an external control panel (52) for user operation. The external control panel controls operating frequencies of the two oscillation valves, the pressure differential, and maximum/minimum pressure. The control panel is supplied by an on/off switch, pressure regulator override, and visual indicator for status of the Pipe-Pulse system components. Power is supplied by an external power supply and distributed to various components by a power distribution system (60). Pressure sensors measure the pressure output at various system locations, as depicted in Figure 6-8, and provide pressure measurement signals to the PLC unit.

The bleed cycle starts when the conduit reaches the maximum preset steady-state pressure. The oscillating valve (26) remains in the open position. While the oscillating valve (36) remains closed and the dump valve is in an open position, the pressure in the bleed line changes by the two-way regulator adjustment to reach a preset $\left(P_{S S}-P_{\text {cyclic }}\right)$ to activate oscillating valve (36), which starts the pressure pulse cycle down. Negative pressure pulses are transmitted in the conduit to decrease pressure in the pipe. During this phase, the two-way regulator is controlled by the PLC to maintain the pressure in the bleed line to within the $\left(P_{S S}-P_{\text {cyclic }}\right)$ range. The oscillating valve (26) closes, when the measured pressure at $\mathrm{P} 4$ falls outside the $\left(P_{S S}-P_{\text {cyclic }}\right)$ range. The measured pressure at $\mathrm{P} 1$ then increases to reach within the $\left(P_{S S}+P_{\text {cyclic }}\right)$ range. When $\mathrm{P} 1$ is within the range, valve 36 oscillation restarts and pressure pulse-down cycles continue. As the pressure in the conduit reaches the minimum preset pressure, both oscillating valves (26 and 36) and the dump valve are closed. The two-way regulator increases pressure P1 until it is within the required range, and a new cycle of the positive pressure pulse transmitted in the conduit is repeated.

\subsubsection{Deployment Safety Aspects, Regulatory Impacts, and Risks Analysis}

The deployment safety aspects, regulatory impacts, and risks analysis for the Pipe-Pulse system are similar in concept to the Hydrokinetics system. This could not be confirmed due to limited information exchange with Paradigm Flow Services. 


\subsection{DEPLOYMENT}

The existence of a plugged waste transfer line leads to general considerations for clearing the line with or without the pressure pulse technology. This section addresses the range of options for preparing and executing the efforts required to clear the line, as shown in Figure 7-1.

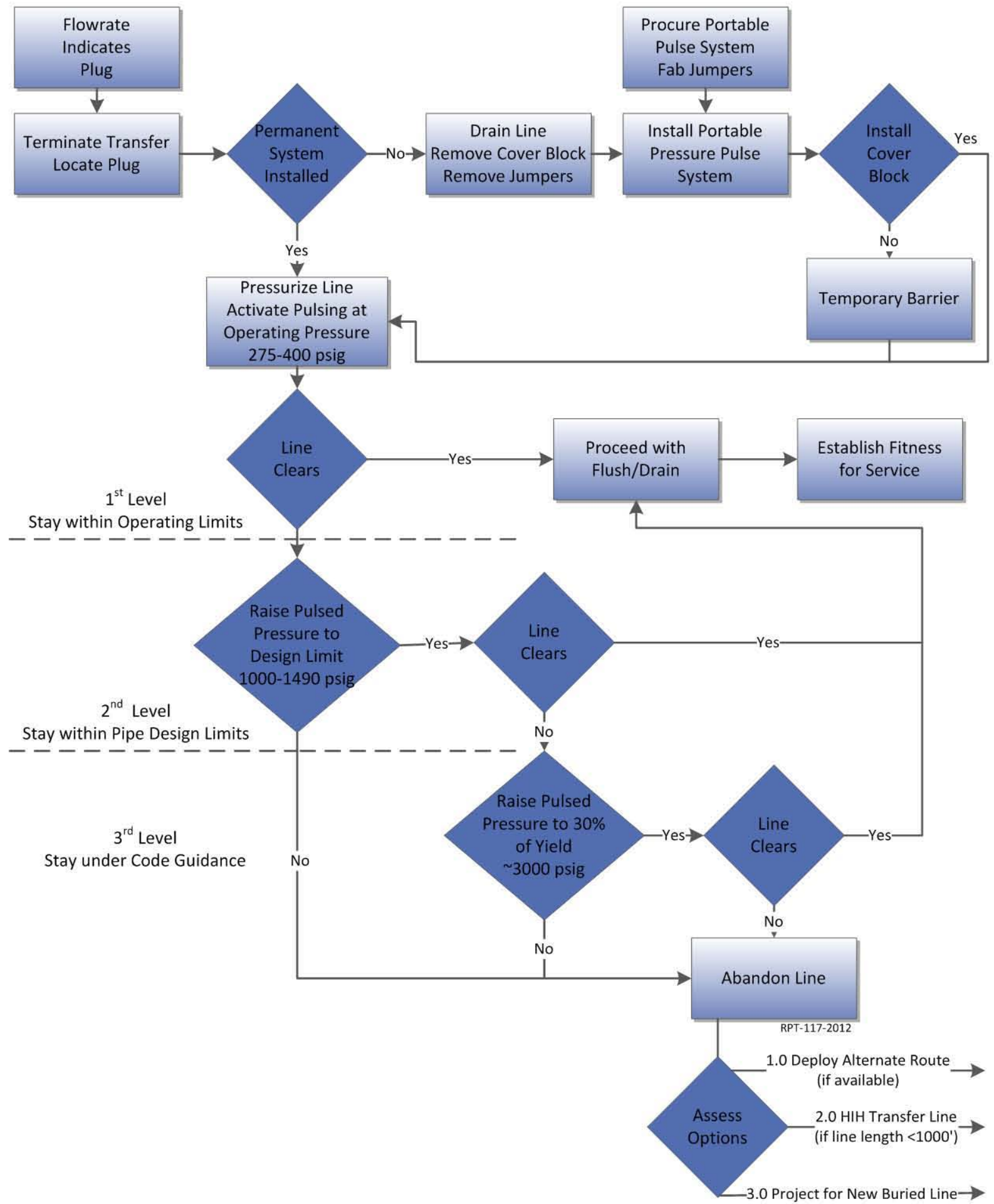

Figure 7-1. Unplugging Strategies 


\subsection{DEPLOYMENT OPTIONS}

The first decision in deploying a pressure pulse system is whether the system used to clear a plugged waste transfer line should be permanently installed as part of the flushing infrastructure or as a portable (modular) unit for application at any pit location.

\subsubsection{Permanent System}

Introducing inhibited water for a pressurized pipeline unplugging effort shares many of the existing functions of the flushing system defined in the DST diluent and flushing specification (HNF-4163), as shown in Figure 7-2. A new diluent and flush system has been designed for the AY/AZ Farm tanks as part of Project T3W12 to meet these specifications. It includes redundant flush pumps, a 17,000-gal raw water queuing tank, metering pumps and tanks for caustic and sodium nitrite, and a heating function to deliver $100^{\circ} \mathrm{F}$ inhibited water. This project scope could be amended during the design phase to include the functions required for unplugging waste transfer lines through pressure pulses and for future applications in AW, AP, AN, and SY Farm tanks. At present, the project cost impact would be limited to a design change. Modifications to the equipment list and building size are not likely to exceed a 20 percent cost impact. It is may also be possible to upgrade the flushing/unplugging capability to $600 \mathrm{psig}$, consistent with hydrostatic testing levels for $400 \mathrm{psig}$ systems.

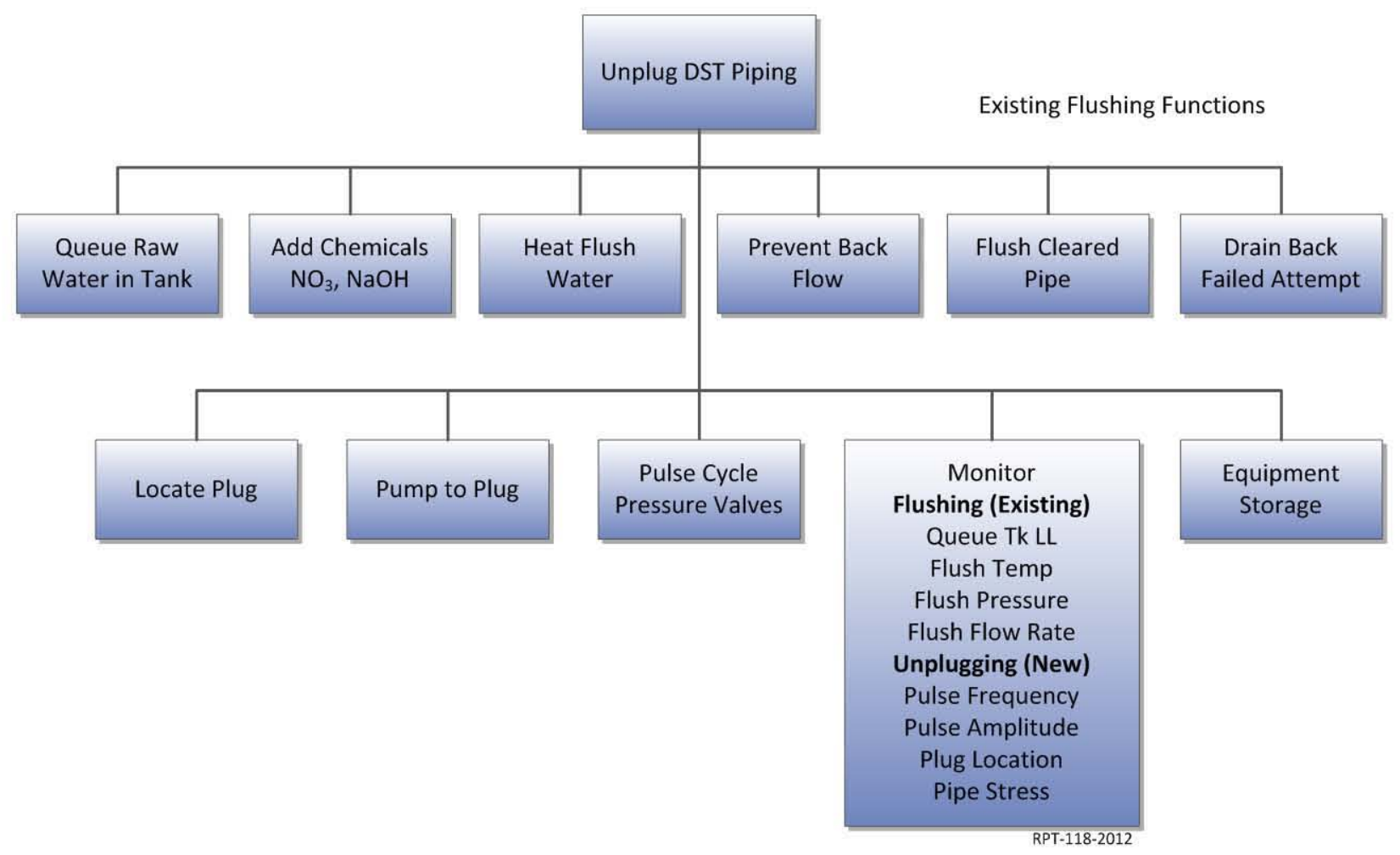

Figure 7-2. Flushing Functions 


\subsubsection{Modular System}

A portable or modular system shares many of the functions used in hydrostatic testing the waste transfer lines. A portable system implies removal of the cover blocks, draining the plugged line, and changing the jumpers. It is likely a temporary barrier would be installed over the pit rather than modifying the cover block to accept the portable unplugging system.

A second option for the portable system that avoids draining the line is to pre-engineer the jumper configuration with a blanked nozzle to connect the pipeline unplugging equipment. The AW Farm valve pit has blanked nozzle connections in two jumpers that could be used for unplugging at 400 or 600 psig, as shown in Figure 7-3. Double-valve isolation would be required to remove the blank and install the pressure pulse system. Another approach would be to access the flush piping outside the valve pit to reach the transfer jumper. The design pressure for the flush piping is a limiting feature. Key points for each approach are summarized in Table 7-1.

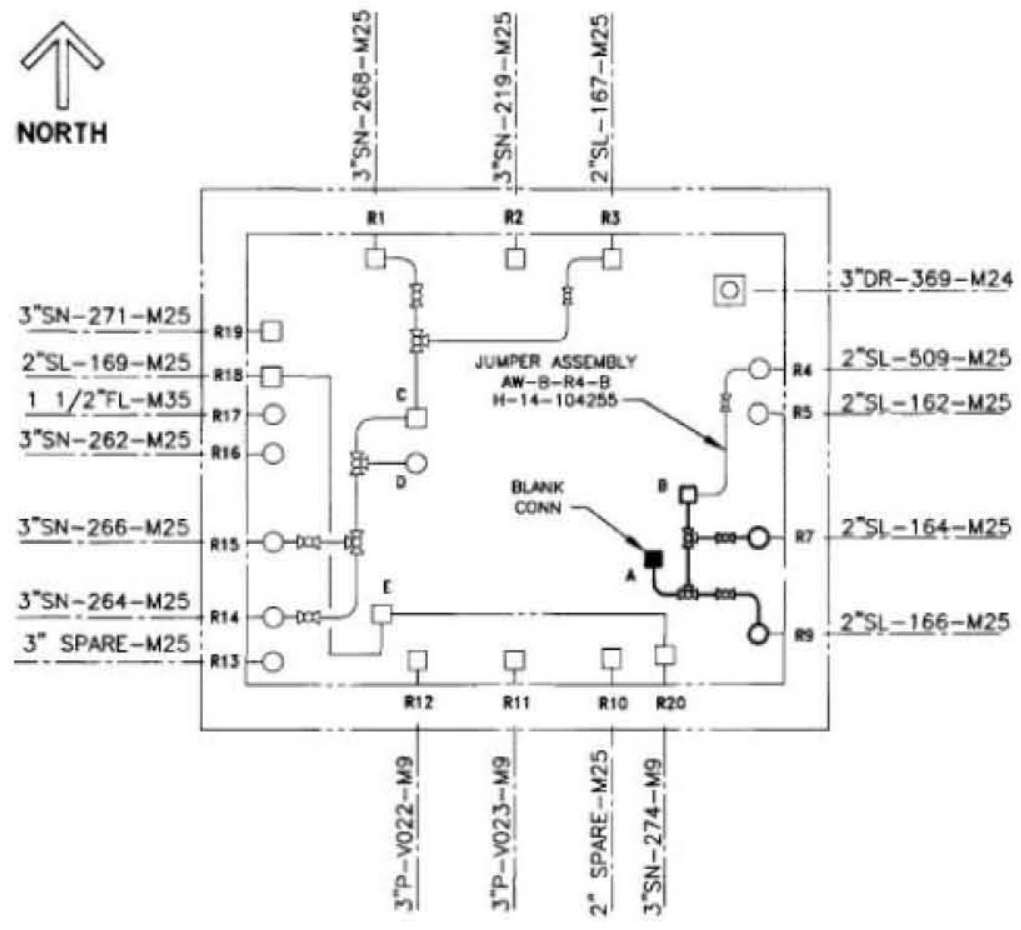

Figure 7-3. Jumper Configuration

\section{Table 7-1. Comparison of Permanent vs. Modular Unplugging Systems}

\begin{tabular}{|c|c|c|}
\hline $\begin{array}{c}\text { Area of } \\
\text { comparison }\end{array}$ & Permanent system & Portable system \\
\hline $\begin{array}{l}\text { Overlapping } \\
\text { functions }\end{array}$ & $\begin{array}{l}\text { Diluents and flush system functions, but is } \\
\text { not available until project completion }\end{array}$ & Hydrostatic testing functions. \\
\hline $\begin{array}{l}\text { Removal of } \\
\text { cover block }\end{array}$ & $\begin{array}{l}\text { Built into jumper system; cover block } \\
\text { removal and line drain not required }\end{array}$ & $\begin{array}{l}\text { Implies removal of cover block, line drained, and } \\
\text { removal of jumpers; requires temporary barrier. }\end{array}$ \\
\hline $\begin{array}{l}\text { Drain plugged } \\
\text { line }\end{array}$ & $\begin{array}{l}\text { Does not require draining the line before } \\
\text { pressurizing for pipeline unplugging } \\
\text { Potentially exposes dozens of other lines via } \\
\text { the valve connections in the pits to damage } \\
\text { or fitness-for-service reviews }\end{array}$ & $\begin{array}{l}\text { Removal of the jumpers does imply draining the } \\
\text { plugged line and thereby introduces the potential for } \\
\text { an air pocket, which may interfere with the pulse } \\
\text { technology making contact with the plug. }\end{array}$ \\
\hline Pressure & $\begin{array}{l}\text { Well-suited for operating pressures; not } \\
\text { readily applicable for higher pressures } \\
\text { because of limiting components in jumpers } \\
\text { and raw line water design pressures }\end{array}$ & $\begin{array}{l}\text { Amenable to higher pressures typically deployed by } \\
\text { the vendor. Means of connecting to the PUREX } \\
\text { nozzle is unknown for pressures above } 600 \text { psig. } \\
\text { However, the cross-site transfer lines were tested at } \\
2,235 \text { psig during construction acceptance testing. }\end{array}$ \\
\hline
\end{tabular}

PUREX = plutonium-uranium extraction . 


\subsubsection{Configuration Summary}

The portable system has the advantage of operating at higher pressures and improving the chances of clearing a plugged line. It is also possible to implement pressure pulse capability in both the permanent flush systems and the portable option for applications with no permanent flush system. An advantageous configuration is one that: (1) does not require draining the transfer line, and (2) can be implemented within hours after the plug occurrence.

A general schematic for the Hydrokinetics system is shown in Figure 7-4. One of the primary modifications for tank farms use is routing the recycle stream for the pulse-down function. The pulse-down would either be accomplished with a manual valve in the pit or by routing the recycle stream from the air-operated pulse-down valve back to the pit drain, as shown in Figure 7-4. This stream is small enough that backflow is not likely to reach the air-operated valves, but safety considerations may require relocating the pulse-down valve or abandoning the pulse-down function in the operating approach.

The pulse-up line is a $1 / 2$-in. or $3 / 4-i n$. hose with a maximum flow of $20 \mathrm{gal} / \mathrm{min}$, the piston pump rate. This configuration would likely remove the possibility of water hammer. Stress modeling and field testing will be used to confirm this assertion addressed by calculation in Section 6.1.

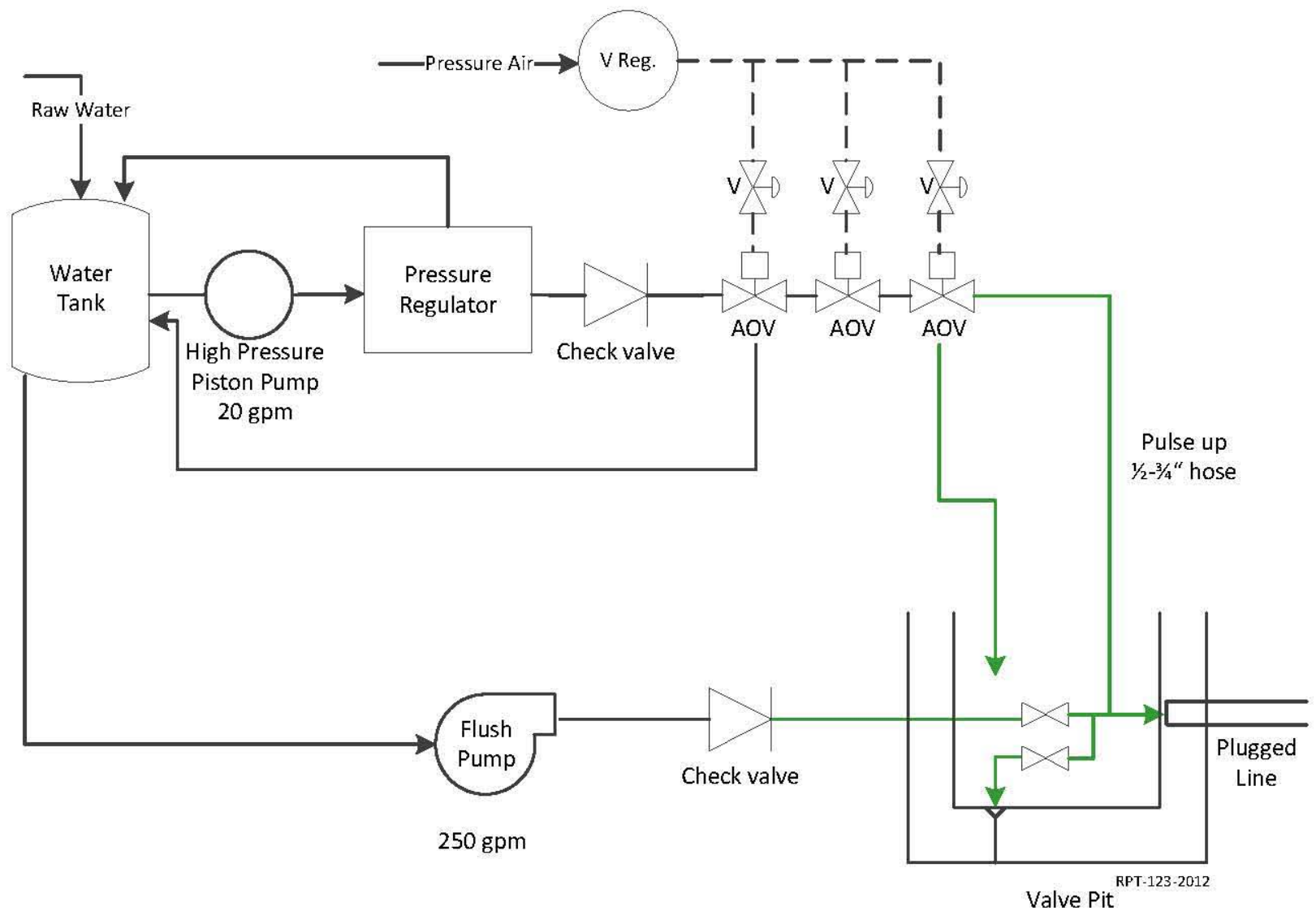

Figure 7-4. Hydrokinetics Modified Process Diagram 


\subsection{MAXIMUM PRESSURE SELECTION}

Loss of a strategic piping route, such as one of the cross-site transfer lines, has significant cost and schedule impacts to the processing mission if an existing alternate route is not available. The option to have a viable waste transfer line unplugging capability warrants serious consideration for pressures at or above 1,000 to $2,000 \mathrm{psig}$, similar to pressures used by the Hydrokinetics system for successful pipeline clearing events in other industries. Use of pressure pulse technologies at 275 to 400 psig operating pressures may prove successful depending on the nature of the plug. However, an option to deploy higher pressures is a strategic advantage, even if the use is never required. The $400 \mathrm{psig}$ systems are pressure-tested with the PUREX connectors at $600 \mathrm{psig}$, providing precedence for unplugging pressures up to $600 \mathrm{psig}$. The Schedule 40 stainless 3 -in. pipe used in the cross-site transfer lines has a design pressure of $1,490 \mathrm{psig}$, has been hydro-tested at $2,235 \mathrm{psig}$ during acceptance testing ( 50 percent above design), and has a burst rating of 9,000 psig. One-third of the burst pressure was used in Figure 7-1 as an upper limit. The maximum pressure decision would not affect the vendorsupplied equipment, which has been used up to 10,000 to $15,000 \mathrm{psig}$. The maximum pump pressure can be specified at lower pressures to comply with safety/code requirements.

The design pressure for key waste transfer line routes is summarized in Table 3-1. Complete design specifications for each specific transfer line are identified in RPP-RPT-52206, Table B.1.

Final selection of the maximum pressure used to unplug a waste transfer line involves weighing the benefits of recovering the line against the risk of damage in the process. The maximum pressure could also be different depending on the transfer line in question.

\subsubsection{Stress Analysis}

A stress analysis is required for all waste transfers in the DST system (TFC-MD-085). The pressure pulse unplugging system would undergo the same rigor for stress analysis to verify the safety basis limits are not exceeded. Stress analysis specifically addresses the dynamic effects of hydraulic shock, including water hammer, surge, and column separation. Relevant issues dealt with in TFC-MD-085 for stress analysis are summarized below.

Safety-significant pressure relief - The potential overpressure conditions resulting from waste transfer pump operations or the dynamic effects of hydraulic shock cannot exceed specified safety basis failure limits. Pressure relieving devices (if present) shall not be credited with limiting pressure in piping, unless they are qualified as safety-significant under tank farms procedural controls (TFC-MD-085, Section $3.2(2 . b)$ ).

Corrosion - For austenitic stainless steel piping, the corrosion-erosion allowance used for pressure design of listed piping components and determining longitudinal stresses in piping systems shall be 1.2 mil per year for the expected service life of the system (TFC-MD-085, Section $3.2(5))$.

Note: This value (1.2 mil per year) is conservative because there is no corrosion data to support a more realistic value. The corrosion allowance is an important factor in stress calculations because of the age of the waste transfer lines. 
Valve operating time - The operating duration of automatically actuated valves shall be assumed to be the actual valve operating time observed for opening or closing during acceptance or operational testing of the integrated waste transfer system. The disturbance time resulting from operating automatically actuated valves shall be based on the assumed operating duration and flow characteristics of the valve types selected (i.e., linear, equal percentage, or quick opening) (TFC-MD-085, Section 3.4 (5.c)).

Stress criteria over pressure allowance - The maximum working pressure resulting from the dynamic effects of hydraulic shock may exceed the design pressure to the extent and for the duration allowed in ASME B31.3, Process Piping, paragraph 302.2.4(f)(2), provided the other criteria in paragraph 302.2.4, subparagraphs (a) through (i) are shown to be met (TFC-MD-085, Section 3.5 (1.c)).

\subsubsection{Stress Calculations}

The water hammer analysis done to-date has shown the jumper valves and valve flanges both have a $300 \mathrm{lb}$ rating, which is consistent with the $400 \mathrm{psig}$ design pressure and 600 psig pressure test for the system (jumpers, valves, PUREX connector, and piping). A single water hammer event, with pressures spiking up to $800 \mathrm{psig}$, was deemed acceptable from a code standpoint (RPP-RPT-52248, Evaluation of Fitness-for-Service for Over-Pressure Events). The limiting features of the system are the valves and associated flanges. If jumpers are taken out of the equation, the transfer line itself could go as high as one-third of yield (2,000-3,000 psig depending on the derating taken for corrosion). The general code guidance is a margin of three under the burst pressure for the pipe alone; approximately 9,000 psig would be derated to approximately $3,000 \mathrm{psig}$. For example, the maximum allowable working pressure for the crosssite transfer line is $1,490 \mathrm{psig}$ (design pressure). A temporary excursion into a higher pressure of twice the design pressure is allowable by code, approximately $3,000 \mathrm{psig}$. If the pressure pulse system is connected directly to the wall nozzle of the plugged line (without jumper components), the higher pressures would be attainable and stress fatigue and corrosion assumptions would become the limiting factors for pressure, in combination with pulse amplitude and frequency. It is likely that a pulsing system for the cross-site transfer line could meet code requirements at pressures up to $1,000 \mathrm{psig}$ for the transfer line alone, with reasonable assumptions for total cycles and corrosion that would determine fatigue. A formal analysis is needed.

The valves in the 6241-A pump station and the 6241-V vent station are special class 900 and have a minimum working pressure of $1,490 \mathrm{psig}$ at $200^{\circ} \mathrm{F}$ (Section 3.3.3.1 of valve specification W-058-P9, As Built Rev. 2 Procurement Specification Air Operated Ball Values). Like the piping, a temporary excursion to pressure above the design rating is possible for the valves.

TFC-ENG-STD-22, Piping, Jumpers, and Valves, sets the design pressure for waste transfer lines at 400 psig. The ASME B31.3 margin on burst pressure is a factor of 3 . The current $A S M E$ Boiler and Pressure Vessel Code (ASME Code 2010), Section III margin for Class 3 is a factor of 3.5 (RPP-RPT-52248). 


\subsubsection{Summary of Pressure Discussion}

The maximum pressure used to unplug a particular waste transfer line is logically predicated on the mechanical properties of the line, the jumper components involved, and the results of the stress analysis assessment for compliance with the safety basis. The pipeline unplugging equipment should be capable of pressures up to 3,000 psig to facilitate a range of choices suited to the properties of individual transfer lines. The Hydrokinetics vendor recommendation for pressure pulsing at Hanford is 1,000 to $1,500 \mathrm{psig}$, while keeping within the allowable pressure range for the pipe (Bradford 2012). The safety and regulatory compliance issues for unplugging are generally satisfied by piping code compliance.

\subsection{CONSEQUENCES OF PLUG MOVEMENT}

Prior to plugging, the soluble salts or insoluble slurries are transferred in solution or in suspension at appropriate concentrations, temperatures, and flow rates to maintain transfer rates. A plug begins with solids build up, a partial line restriction, and eventual pump shutoff. The parameters that cause plugging are normally gradual over several hours, and partially restricted flow is evidenced in the control room as reduced flow rate and elevated pump pressures and amperage. The first operator response to eliminate the plug is to increase the dilution rate (usually at the pump intake) or flush the line with heated water.

If a plug still occurs, the plug volume is typically small, relative to the line volume. The power requirements to establish a large volume plug are not available. Head pressures on the pump side of a plug exceed the pump capability or pressure limitations on the piping early in the phase change from higher viscosities to plugging. Maintaining $6 \mathrm{ft} / \mathrm{sec}$ for slurry transfers exceeds the 400 psig design limit for longer transfer lines unless densities are kept under 1.3 (RPP-5346, Waste Feed Delivery Transfer System Analysis). A line plug is likely to be a partial blockage (not airtight) before the pump exceeds amperage or pressure limitations and shuts down automatically.

The plug location is not normally known because the waste transfer lines are buried. Plugs presumably form at a distant cool point where temperatures drop off enough to cause a significant viscosity change.

It is possible (but not likely) to move an entire plug as an intact entity during pressure pulsing and reform the plug in a restriction point. The pipelines have long radius elbows in the expansion joints. Likely restriction points are the multiple 90-degree bends in the PUREX connectors used for the jumpers in the receiving pit. In the event a plug is moved intact into a jumper network, the solution is to open the pit and remove and flush the jumpers. Another strategy is to simply remove the downstream jumpers so that the plug is flushed into the valve pit and drained into the DST associated with the receiving pit. The process of finding the plug in the transfer line may entail flushing individual portions of the route followed by removal of the jumpers prior to attempts at unplugging the line.

The likely scenario after a plug is broken by pressure pulsing is to redissolve or resuspend the solids in the flush water. Once the plug is broken and flow is reestablished, the turbulence acts to mix and thereby enhance breakup, entrainment, and dissolution of any solids. 


\subsection{COST AND SCHEDULE}

\subsubsection{Permanent Facility Costs}

The AY/AZ diluent and flush system provided by Project T3W 12 is fully designed but construction may not be completed to prioritize early LAW feed delivery. The project estimate shown in Table 7-2 provides a basis for approximating a permanent facility. The P\&ID is shown in Figure 7-5, and the facility is shown in Figure 7-6.

Adding a built-in pressure pulse capability implies the need for a high-pressure pump, an accumulator tank, pulse valves, and additional instrumentation and controls. For this study, an estimate for the permanent pressure pulse system of an additional 20 percent would put the total project cost at $\$ 5$ million.
Table 7-2. AY/AZ Farm Diluent and Flush Facility Project T3W12

\begin{tabular}{|l|r|}
\multicolumn{1}{|c|}{ Project Component } & $\begin{array}{r}\text { Estimated } \\
\text { Cost (\$K) }\end{array}$ \\
\hline Retaining wall/stairs/slab & 474 \\
\hline Procure 17,500-gal tank & 211 \\
\hline $\begin{array}{l}\text { Procure/install mechanical, includes } \\
\text { caustic and nitrite tanks and metering } \\
\text { system }\end{array}$ & 1,457 \\
\hline Procure/install electrical/instrumentation & 576 \\
\hline $\begin{array}{l}\text { Procure in-line heaters } \\
\text { Fabricate/install building structure, roof } \\
\text { portion is \$201K }\end{array}$ & 446 \\
\hline Mechanical testing & 500 \\
\hline $\begin{array}{l}\text { Electrical testing } \\
\text { Total (fully burdened including WRPS } \\
\text { field support) }\end{array}$ & 159 \\
\hline
\end{tabular}

Source: Kraemer, K. E., 2012, "Diluent and Flush System Building Costs," (email to J. S. Garfield, October 19), BNL Technical Services, LLC, Richland, Washington (attached as Appendix F)

WRPS = Washington River Protection Solutions, LLC. 


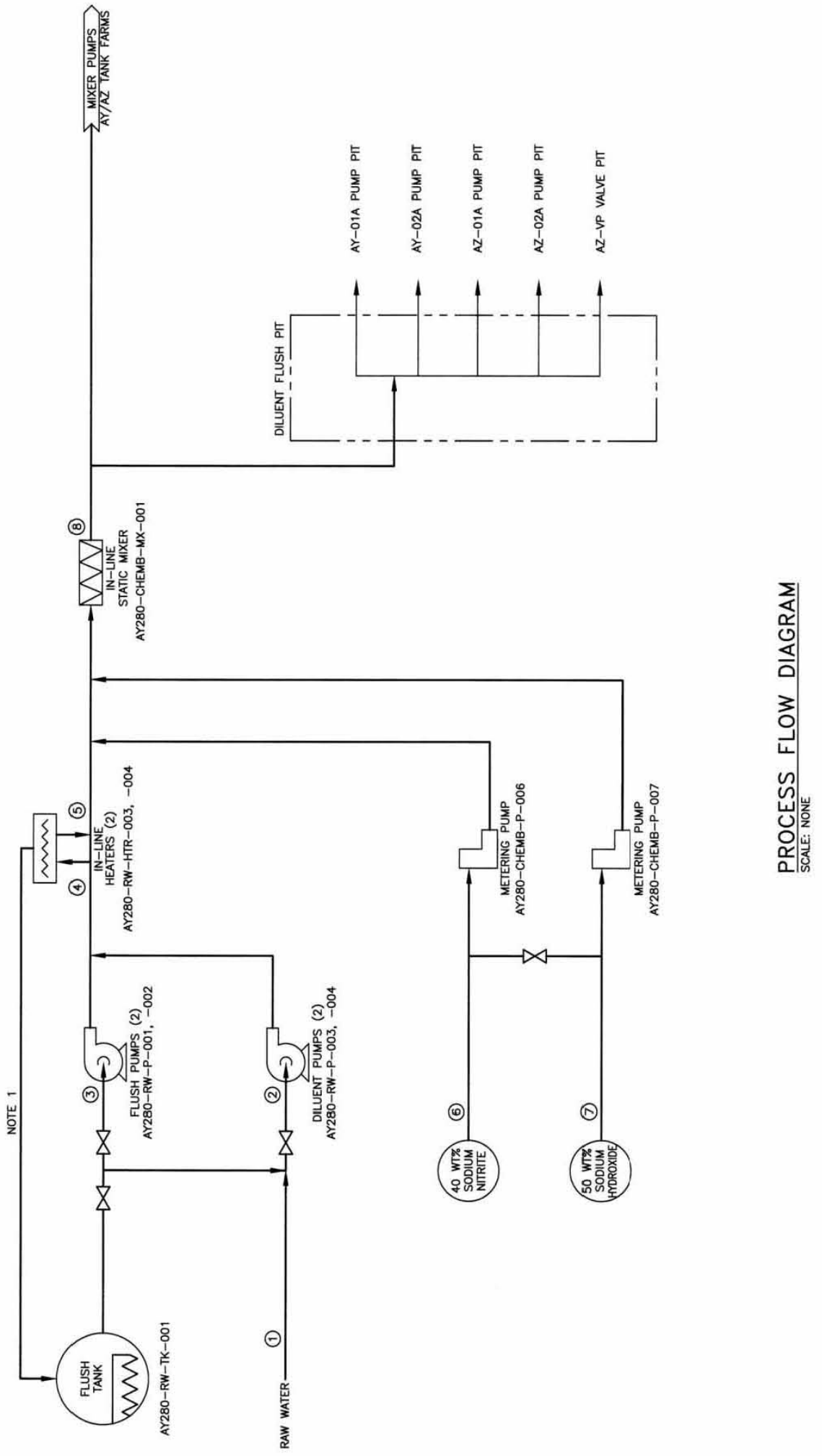

Source: H-14-109301, "Process Infrastructure Upgrades Process Flow Diagram,” Sheet 1, Rev. 0.

Figure 7-5. AY/AZ Farm Diluent and Flush System Piping and Instrumentation Diagram 


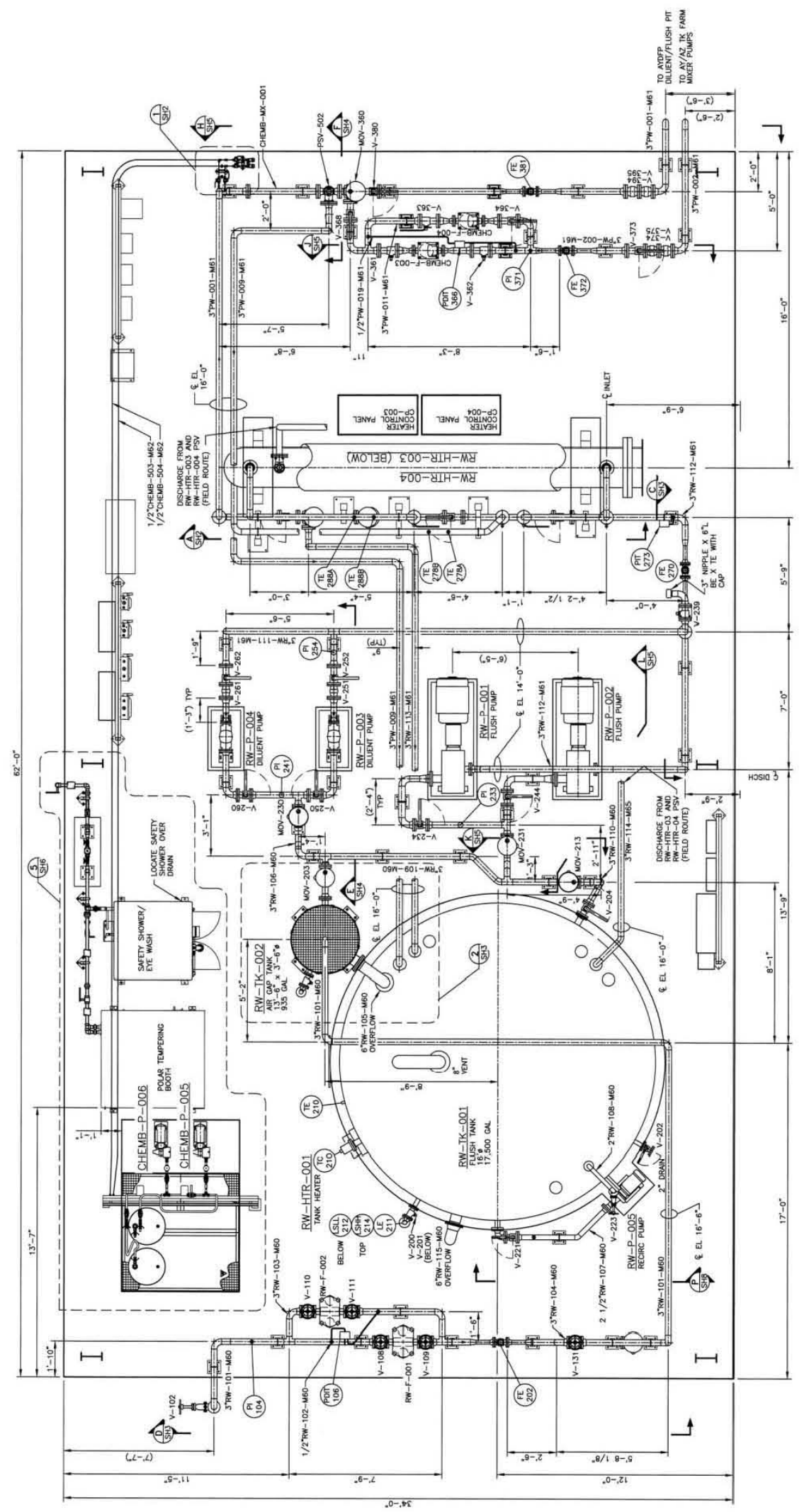

Source: H-14-109033, 2011, "Mechanical Diluent/Flush Pad Piping Plan," Sheet 1, Rev. 0.

Figure 7-6. AY/AZ Farm Piping Plan 


\subsubsection{Portable Facility Costs}

Cost estimates and specifications for a trailer-mounted, high-pressure piston pump, motor, and valves similar to the Hydrokinetics system are yet to be estimated in detail. The entire package (Hydrokinetic equipment, hydroblast pump, hoses, accessories, etc.) was sold once in the history of the company for $\$ 512,000$. The typical charge rate for non-nuclear, on-site service is $\$ 8,000$ to 20,000 per shift, depending on the application (Bradford 2012, Appendix G).

The approach at Hanford would involve buying the equipment and having AIMM Technologies provide the training or oversight of the unplugging operation. It is possible to allow the vendorowned equipment into the tank farms as a paid service, but for safety, operational and liability issues, the equipment will be purchased, retained onsite, and operated by WRPS plant forces. 


\subsection{ALTERNATIVE COMPARISON}

Comparison of the two vendor alternatives (Pipe-Pulse and Hydrokinetics) was not possible, or necessary, as the engineering investigation progressed for several reasons:

- Paradigm Flow Services, Ltd would not divulge any of the details of their Pipe-Pulse technology without a rigorous nondisclosure agreement. A business decision was made, with concurrence from WRPS, to not sign the agreement. As a result, the information on the Pipe-Pulse technology is limited to what was publicly available in patents and published reports.

- AIMM Technologies, Inc. did share their operating approach for the Hydrokinetics technology, but also requested a nondisclosure agreement before sharing the operational approach summarized in Section 6.3.1.

- Both technologies appear to be very close to the same approach.

Explanations of the operating principle behind the standing wave, frequency, amplitude, and cavitation are not well substantiated with engineering evidence. It does appear that the combination of pressure, the standing wave, and pulsing at 2-30 cycles per minute does work in the field. The pulses to the static liquid head are well below the level of water hammer. However, acoustic cavitation between the plug and pipe wall probably are a benefit of the standing wave introduced by the pressure pulse system. The Pipe-Pulse process reportedly operates below cavitation with an automated system using sophisticated algorithms in the public literature. The Hydrokinetics process reportedly uses the positive benefits of acoustic cavitation with a manual approach. The field experience with the Hydrokinetics technology does not point to issues with pipe vibration. The Hydrokinetics system is often used with above grade piping systems and mechanical vibration is not seen from the pulsing. The pipeline plugs often show separation at the wall interface, suggesting that cavitation is a factor.

Given the present level of detail on the technical approach, the two vendor technologies are similar enough that they cannot be distinguished in terms of how they would be deployed in a tank farms setting. Safety, regulatory issues, cost, and risk are also comparable. The use of cavitation is an advantage for the Hydrokinetics technology, but neither vendor nor past testing at FIU has shown conclusive evidence that cavitation is or is not a factor. Field operations using Hydrokinetics have successfully unplugged lines with a high degree of success. Hydrokinetics users are often repeat customers, and there is no particular business incentive to confirm the theory of cavitation.

The Hydrokinetics technology was used in the engineering evaluation performed for this study. The options for deployment (portable vs. permanent) and the maximum pressures used are the primary variables for implementation at Hanford. 


\subsection{CONCLUSIONS}

The following conclusions were derived from this study.

Integration with Flushing - The waste transfer line unplugging strategy should be integrated with the chemical waste type involved in each waste transfer and the flushing strategy. There are four basic types of possible plugging issues. The basic chemistry of the plug influences both the transfer line flushing and unplugging efforts. The response to a plug should be preplanned such that the engineered solution and implementation approach are known and can be implemented without delay (e.g., waiting for approvals).

Technology Maturity - The pressure, frequency, and amplitude requirements of the pressure pulse technology has not been optimized at this time for Hanford waste transfer line application. In addition, different waste transfer lines have different thresholds for stress, depending on the original design pressure and corrosion assumptions due to age. However, the pressure pulse equipment can be easily configured for a range of pressures and pulse frequencies. This approach allows development testing at FIU to progress and for transfer line-specific structural analysis to be completed. Implementation of a pressure pulse technology would begin at lower pressures and amplitude and work up to optimal unplugging process values, with maximums defined by the structural analysis.

Portable vs. Permanent - A portable (modular) pressure pulse system should be developed for use in any tank farms pit where a plug might occur. A permanent pressure pulse system can be considered for high use locations where the flush system is being upgraded. In particular, the cross-site transfer lines originating in the SY Farm valve pits should be upgraded to a flush and pulse system rated for the same pressures as the cross-site transfer line (1,490 psig). A permanent system would also integrate with an automatic flushing function in the event of a pump failure during a waste transfer.

Air Pockets - A pressure pulse technology offers no benefit at the face of a plug, if an air pocket is hydraulically above the pressure source. Every transfer line has a minimum 0.25 percent slope in one direction between pits. The pressure pulse source must be applied at the high end pit. In theory, the plug would prevent the waste transfer line from draining, while the pressure pulse equipment is put in place. In the event the waste transfer line is not full, the ability to fill the line slowly, to maintain channel flow to the plug face, and to bleed off excess air needs to be part of the functional capability of the unplugging process.

The cross-site transfer lines slope up from both ends to a mid-point. After a successful transfer, air is bled into the lines to facilitate draining. In a plug scenario, the waste transfer lines are pulsed before draining or drained to remove the jumpers and refilled with water through the $6241-\mathrm{V}$ vent station. Both options require design changes to the jumpers in the SY Farm valve pits or the piping at the vent station. A third scenario is to pressurize the drained transfer line, assuming air can be forced past the plug. 


\subsection{RECOMMENDATIONS}

Develop the unplugging strategy - Figure 10-1 summarizes the key decisions associated with transfer line unplugging. Currently, there is no pre-planned strategy for a response to a line plug. The first recommendation is to develop a logical and safe approach for unplugging that can be implemented without the schedule delays required for defining the approach, gaining approvals, and preparing procedures.

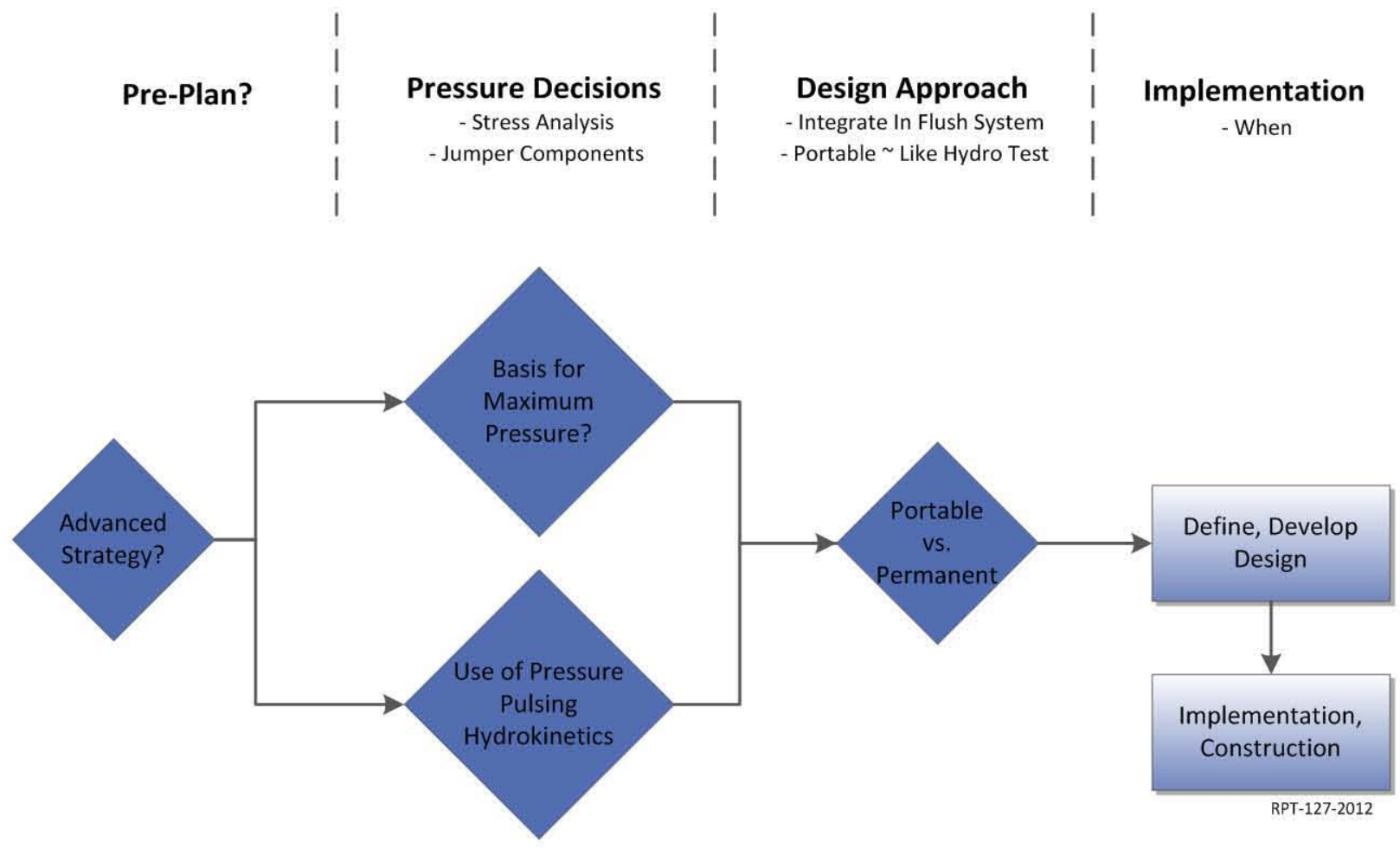

Figure 10-1. Transfer Line Unplugging Decisions

Pressure decisions - The second major decision is determining the maximum pressure that can be used on a given transfer line. Stress analysis can be used to provide a basis for both the maximum pressure and the effects of pressure pulsing.

WRPS has contracted Becht Engineering ${ }^{13}$ to conduct a water hammer study that models selected waste transfer line routes in the 200 East Area in three dimensions. Procedures are being put in place to assess every waste transfer for the dynamic effects of hydraulic shock, including water hammer, surge, and column separation. Investigating the structural impacts from pulsing the transfer lines for unplugging will be required before implementation and will support the downselection of pressure pulse amplitude and frequency. The analysis will cost about $\$ 15-50 \mathrm{~K}$ to model a range of frequencies and amplitude for both structural damage and attenuation issues for the 200 East Area waste transfer lines already modeled. Field experience could provide an empirical data point for the stress issue associated with the pressure pulse technology. Water hammer or vibration has not been observed in the field at any amplitude or frequency.

\footnotetext{
${ }^{13}$ The Becht Engineering contact is Jack Cole.
} 
Jumper components - The valves and PUREX connectors included in the jumpers must be specified and included in the pressure assessment if the jumpers are left in place for unplugging.

The PUREX connector is currently rated at 400 psig. Although it is used at $600 \mathrm{psig}$ in hydrostatic testing, its rating should be improved or another connector designed for use in unplugging the waste transfer lines. The design pressure should be 1,000-1,500 psig, and the sharp 90-degree bend eliminated.

Design approach - Design and development of the pressure pulse technology for use at Hanford to address waste transfer line plugging should continue based on vendor experience in the field for non-nuclear applications and the results of piping stress analysis. The technology should be implemented in two phases.

1. A portable pressure pulse system should be developed and procured for implementation that is similar in principle to the hydrostatic testing process currently used at Hanford. This system could be used at any tank farm location or valve pit. The trailer-mounted equipment could also be configured to service both hydrostatic testing and unplugging needs in the tank farms.

2. The current flushing system upgrades planned for AY/AZ and AW Farm tanks should be reviewed to include a pressure pulse unplugging function at pressures allowed by the stress analysis (approximately 1,000 psig). The combined functions would allow immediate high-pressure unplugging attempts for strategic waste transfer lines needed to support WTP waste feed delivery. A permanent flushing/unplugging capability should be used in conjunction with the SY Farm valve pits for the cross-site transfer lines.

Implementation - The decision on when to implement the unplugging strategy and have the equipment available for use is linked to the operational need:

- Ongoing transfers support current retrieval and evaporator campaigns

- Slurry transfers associated with Tank AY-102 retrieval

- WTP feed queuing and transfers.

The cost for implementing a portable system is relatively inexpensive and provides insurance for the risk of a transfer line plug. It is may also be reasonable to use the same trailer-mounted equipment for hydrostatic testing in the near-term. It is recommended that the portable system be designed and implemented to support near-term operations. Ongoing refinements to the pulsing strategy can be accommodated within the control scheme for pulse amplitude and frequency. The pulsing function is a not a cost driver.

An assessment of the transfer lines should be conducted to identify the lines most used for slurry transfers that do not have a ready backup or alternative. OR modeling of the impacts to the mission from the loss of these lines should be pursued.

A formal functions and requirements document should be developed to support further equipment development and to guide the FIU work. 
FIU interface - DOE funded FIU to support transfer line unplugging efforts at all DOE sites. Dr. Dwayne McDaniel (the FIU point of contact) was very receptive to addressing Hanford needs within the platform of their existing funding and scope. WRPS should provide a formal point-of-contact to represent Hanford needs. Some of the specific areas for consideration include:

- Testing pipe (2- and 3-in. Schedule 40 stainless and carbon steel) with the same mechanical properties and configuration over greater distances.

- Does cavitation occur as the operating principle to move plugs from the pressure wave induced by the Hydrokinetics process?

- Can the pressure pulse system remain effective for two to three miles? At what pressures?

- Do expansion joints or air pockets attenuate the unplugging properties of the pulse wave at distance?

- Are there harmonics that could structurally damage the pipe/expansion joints?

- What are the optimal amplitude and frequency recommended for unplugging the waste transfer lines?

- Is there a pulse echo technology that can be used to monitor the strength of the pulse wave? 


\subsection{REFERENCES}

24590-WTP-GPG-M-0059, 2007, Design Guide: Avoiding Chemical Line Plugging Plant Design Considerations, Rev. 0, Bechtel National, Inc., Richland, Washington.

24590-WTP-RPT-PT-02-005, 2011, Flowsheet Bases, Assumptions, and Requirements, Rev. 6, Bechtel National, Inc., Richland, Washington.

ANSI/ISEA Z358.1, 2009, American National Standard for Emergency Eyewash and Shower Equipment, International Safety Equipment Association, Arlington, Virginia.

ASME B31.3, 2012, Process Piping, American Society of Mechanical Engineers, New York, New York.

ASME Code, 2010, ASME Boiler and Pressure Vessel Code, Section III, "Rules for Construction of Nuclear Power Plant Components, Division 1, Subsection ND: Class 3 Components," American Society of Mechanical Engineers, New York, New York.

ASTM A53, 2012, Standard Specification for Pipe, Steel, Black and Hot-Dipped, Zinc-Coated, Welded and Seamless, American Society for Testing and Materials, West Conshohocken, Pennsylvania.

ASTM A106, 2011, Standard Specification for Seamless Carbon Steel Pipe for HighTemperature Service, American Society for Testing and Materials, West Conshohocken, Pennsylvania.

ASTM A312, 2012, Standard Specification for Seamless, Welded, and Heavily Cold Worked Austenitic Stainless Steel Pipes, American Society for Testing and Materials, West Conshohocken, Pennsylvania.

ASTM D2996, 2007, Standard Specification for Filament-Wound "Fiberglass" (Glass-FiberReinforced Thermosetting-Resin) Pipe, American Society for Testing and Materials, West Conshohocken, Pennsylvania.

ASTM D2997, 2007, Standard Specification for Centrifugally Cast "Fiberglass" (Glass-Fiber-Reinforced Thermosetting-Resin) Pipe, American Society for Testing and Materials, West Conshohocken, Pennsylvania.

Bain, R., 2012, "Pipe-Pulse Technology for Subsea Blockage Removal," pp. 28-33, Drilling and Exploration World (DEW) Journal, March, International Edition, Vol. 21, No. 05.

Bradford, B., Jr., 2012, "RE: Follow up Questions about the Hydrokinetics System," (e-mail to J. Garfield, Washington River Protection Solutions, LLC, November16), AIMM Technologies, Inc., Texas City, Texas.

Chesshyre, M., 2011, "Paradigm Shift," Offshore Engineer, September 1, online at http://atcomedia.com/sites/oedigital.com/cms/k2-cat/item/428-paradigm-shift.

DOE/EIS-0189, 1996, Tank Waste Remediation System, Hanford Site, Richland, Washington, Final Environmental Impact Statement, U.S. Department of Energy, Richland Operations Office, Richland, Washington.

DOE/EIS-0391, 2012, Final Tank Closure and Waste Management Environmental Impact Statement for the Hanford Site, Richland, Washington (TC \& WM EIS), U.S. Department of Energy, Office of River Protection, Richland, Washington. 
FIU, 2009, Unplugging of High Level Waste Transfer Pipelines: AIMM Technologies' Hydrokinetic Process Technology Assessment, Florida International University, Miami, Florida (attached as Appendix B).

Gokaltun, S., T. Pribanic, J. Varona, D. McDaniel, A. Awwad, and D. Roelant, 2010, "Evaluation and Development of Innovative High-Level Waste Pipeline Unplugging Technologies," Paper 10136, 2010 Waste Management Conference, March 7-10, Phoenix, Arizona.

H-2-822209, 1995, "Civil General Notes and Details," Sheet 1, Rev. 3, ICF Kaiser Hanford Company, Richland, Washington.

H-2-822214, 1995, "Civil Plan and Profile STA 54+ to 72+," Sheet 1, Rev. 2, ICF Kaiser Hanford Company, Richland, Washington.

H-14-103240, 2004, "Civil SN-636, SN-637 \& SN-634 STA 14+25 to End," Sheet 1, Rev. 4, U.S. Department of Energy, Office of River Protection, Richland, Washington.

H-14-109033, 2011, "Mechanical Diluent/Flush Pad Piping Plan," Sheet 1, Rev. 0, U.S. Department of Energy, Office of River Protection, Richland, Washington.

H-14-109301, "Process Infrastructure Upgrades Process Flow Diagram," Sheet 1, Rev. 0, U.S. Department of Energy, Office of River Protection, Richland, Washington.

Herting, D. L., 1980, "Evaporator Feeds High in Phosphate," (internal letter 65453-80-296 to D. A. Reynolds, October 9), Rockwell Hanford Operations, Richland, Washington.

Herting, D. L., 2005, "Phosphate Solubility Under C Farm Retrieval Conditions," (interoffice memo 7S110-DLH-05-027 to D. M. Nyugen, July 18), CH2M HILL Hanford Group, Inc., Richland, Washington.

HNF-4157, 2011, Double-Shell Tank Utilities Subsystem Specification, Rev. 4, Washington River Protection Solutions, LLC, Richland, Washington.

HNF-4160, 2011, Double-Shell Tank Transfer Valving Subsystem Specification, Rev. 5, Washington River Protection Solutions, LLC, Richland, Washington.

HNF-4161, 2011, Double-Shell Tank Transfer Piping Subsystem Specification, Rev. 5, Washington River Protection Solutions, LLC, Richland, Washington.

HNF-4163, 2011, Double-Shell Tank Diluent and Flush Subsystem Specification, Rev. 6, Washington River Protection Solutions, LLC, Richland, Richland, Washington.

Kraemer, K. E., 2012, "Diluent and Flush System Building Costs," (email to J. S. Garfield, October 19), BNL Technical Services, LLC, Richland, Washington (attached as Appendix F).

Morris, M. I., J. L. Ladd-Lively, and B. E. Lewis, 2004, Pipeline Unplugging Assessment and Recommendations for Fernald Environmental Management Project, Nuclear Science and Technology Division, Oak Ridge National Laboratory, Oak Ridge Tennessee (Section 3.5 and Appendix I attached as Appendix D).

National Environmental Policy Act of 1969, 42 USC 4321, et seq.

OR-78-88, 1978, Occurrence Report: Plugged Cross-Country Transfer Line, Rockwell Hanford Operations, Richland, Washington. 
ORNL/TM-2000/298, 2000, Waste Preparation and Transport Chemistry: Results of the FY 2000 Studies, Oak Ridge National Laboratory, Oak Ridge, Tennessee.

PNNL-11103, 1997, Retrieval Process Development and Enhancement Project Fiscal Year 1995 Simulant Development Technology Task Progress Report, Pacific Northwest National Laboratory, Richland Washington.

PNNL-11497, 1997, Cross-Site Transfer System at Hanford: Long-Term Strategy for Waste Acceptance, Pacific Northwest National Laboratory, Richland Washington.

PNNL-11685, 1997, Retrieval Process Development and Enhancements Waste Simulant Compositions and Defensibility, Pacific Northwest National Laboratory, Richland Washington.

PNNL-17758, 2008, Preparation and Characterization of Chemical Plugs Based on selected Hanford Simulants, Pacific Northwest National Laboratory, Richland Washington.

RCW 70.105, "Hazardous Waste Management Act of 1976," Revised Code of Washington, Washington State Department of Ecology, Olympia, Washington.

Resource Conservation and Recovery Act of 1976, 42 USC 6901, et seq.

RPP-5346, 2002, Waste Feed Delivery Transfer System Analysis, Rev. 2, CH2M HILL Hanford Group, Inc., Richland, Washington.

RPP-10905, 2002, Recommended Responses to a Postulated HLW Feed Delivery Pipeline Plug, Rev. 0, CH2M HILL Hanford Group, Inc., Richland, Washington.

RPP-13033, 2012, Tank Farms Documented Safety Analysis, Rev. 4-Q, Washington River Protection Solutions, LLC, Richland, Washington.

RPP-15136, 2006, System Design Description for the Replacement Cross-Site Transfer System Between 200 West and 200 East Tank Farms, Rev. 3, CH2M HILL Hanford Group, Inc., Richland, Washington.

RPP-15137, 2011, System Design Description for the 200 East Area DST Waste Transfer System (DSA-Based), Rev. 6, Washington River Protection Solutions, LLC, Richland, Washington.

RPP-17247, 2003, Dilution and Flushing Requirements to Avoid Solids Precipitation and Deposition during Tank Waste Transfers, Rev. 0, CH2M HILL Hanford Group, Inc., Richland, Washington.

RPP-18652, 2006, Buried Pipe Analysis for DST System Integrity Assessment, Rev. 1, CH2M HILL Hanford Group, Inc., Richland, Washington.

RPP-25113, 2006, Residual Waste Inventories in the Plugged and Abandoned Pipelines at the Hanford Site, Rev. 0-A, CH2M HILL Hanford Group, Inc., Richland, Washington, Richland, Washington.

RPP-46506, 2011, Decision Framework Key Decisions Required to Achieve the RPP Mission, Rev. 2, Washington River Protection Solutions, LLC, Richland, Washington.

RPP-RPT-42297, 2012, Safety-Significant Waste Transfer Primary Piping Systems - Functions and Requirements Evaluation Document, Rev. 10, Washington River Protection Solutions, LLC, Richland, Washington. 
RPP-RPT-46112, 2010, Transfer Line Reliability for the Waste Feed Delivery Operations Research Model Phase 2, Rev. 0, Washington River Protection Solutions, LLC, Richland, Washington.

RPP-RPT-47572, 2012, Cross-Site Slurry Line Evaluation Report, Rev. 0, Washington River Protection Solutions, LLC, Richland, Washington.

RPP-RPT-51921, 2012, The Phase 3.1 Hanford Waste Feed Delivery Operations Research Model Bases and Assumptions, Rev. 0, Washington River Protection Solutions, LLC, Richland, Washington.

RPP-RPT-52206, 2012, Tank Farms Waste Transfer System Fitness-for-Service Requirements and Recommendations, Rev. 0, Washington River Protection Solutions, LLC, Richland, Washington.

RPP-RPT-52248, 2012, Evaluation of Fitness-for-Service for Over-Pressure Events, Rev. 0-A, Washington River Protection Solutions, LLC, Richland, Washington.

RPP-RPT-52347, 2012, Pipeline Unplugging Assessment and Recommendations for Hanford Waste Transfer Systems, Rev. 0, Washington River Protection Solutions, LLC, Richland, Washington.

TFC-ENG-DESIGN-C-47, 2012, Process Hazard Analysis, Rev. A-2, Washington River Protection Solutions, LLC, Richland, Washington.

TFC-ENG-STD-22, 2012, Piping, Jumpers, and Valves, Rev. E-7, Washington River Protection Solutions, LLC, Richland, Washington.

TFC-ENG-STD-26, 2012, Waste Transfer, Dilution, and Flushing Requirements, Rev. A-10, Washington River Protection Solutions, LLC, Richland, Washington.

TFC-MD-085, 2012, Design Conditions for Analyses of Process Piping, Rev. D, Washington River Protection Solutions, LLC, Richland, Washington.

TFC-PLN-39, 2011, Risk and Opportunity Management Plan, Rev. G, Washington River Protection Solutions, LLC, Richland, Washington.

W-058-P9, 1998, As Built Rev. 2 Procurement Specification Air Operated Ball Values, Rev. 1, Lockheed Martin Hanford Corporation, Richland, Washington.

WAC 173-303, "Dangerous Waste Regulations," Washington Administrative Code, as amended.

WAC 246-290, "Public Water Supplies," Washington Administrative Code, as amended.

Weast, R. C, 1974-1975, CRC Handbook of Chemistry and Physics, 55 ${ }^{\text {th }}$ Edition, page E-47, CRC Press, Cleveland, Ohio. 
RPP-RPT-53783, Rev. 0

Appendix A

HYDROKINETICS PATENT 


\section{United States Patent}

Garcia
[54] METHOD AND APPARATUS FOR

CLEANING COLUMNS BY INDUCING VIBRATIONS IN FOULING MATERIAL AND THE COLUMN

[75] Inventor: Patricia M. Garcia, Houston, Tex.

[73] Assignee: American International, Inc, Houston, Tex.

[21] Appl. No.: 489,552

[22] Filed: Jun. 12, 1995

\section{Related U.S. Application Data}

[63] Continuation-in-part of Ser. No. 16,855, Feb. 12, 1993, Pat. No. $5,423,917$.

[51] Int. $\mathbf{C l} .^{6}$

B08B 3/12; B08B 9/02

[52] U.S. Cl. $134 / 1 ; 134 / 22.11 ; 134 / 22.12$ $134 / 167 \mathrm{C} ; 134 / 169 \mathrm{C}$

[58] Field of Search $134 / 1,22.11,22.12$ $134 / 167$ C, 169 C, 166 C
[11] Patent Number:

$5,674,323$

[45] Date of Patent:

Oct. 7, 1997
$[56]$

\section{References Cited \\ U.S. PATENT DOCUMENTS}

\begin{tabular}{|c|c|c|}
\hline 457,108 & $7 / 1969$ & Hittel ....... \\
\hline $3,565,589$ & $2 / 1971$ & Lowe et al. \\
\hline 6,623 & $7 / 1985$ & ......... 134/22.12 X \\
\hline 4,7 & $2 / 1988$ & .. $134 / 1$ \\
\hline & 11/ & , \\
\hline & $2 / 1$ & $34 / 22.12$ \\
\hline 54 & 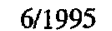 & $134 / 1$ \\
\hline
\end{tabular}

Primary Examiner--Jill Warden Assistant Examiner-Saeed Chaudhry

[57]

ABSTRACT

A method of cleaning in a pipe which has fouling material on the interior so that the bond with the fouling material is broken by first filling the pipe with liquid, applying pressure pulsations to the liquid to the extent that a standing wave is formed in the pipe and the incompressible liquid therein, and cavitation is initiated within the standing wave in the pipe so that the pipe is cleaned on the formation of induced shock waves occurring upon collapse of microscopic bubbles resulting from cavitation and subsequent collapse.

16 Claims, 2 Drawing Sheets

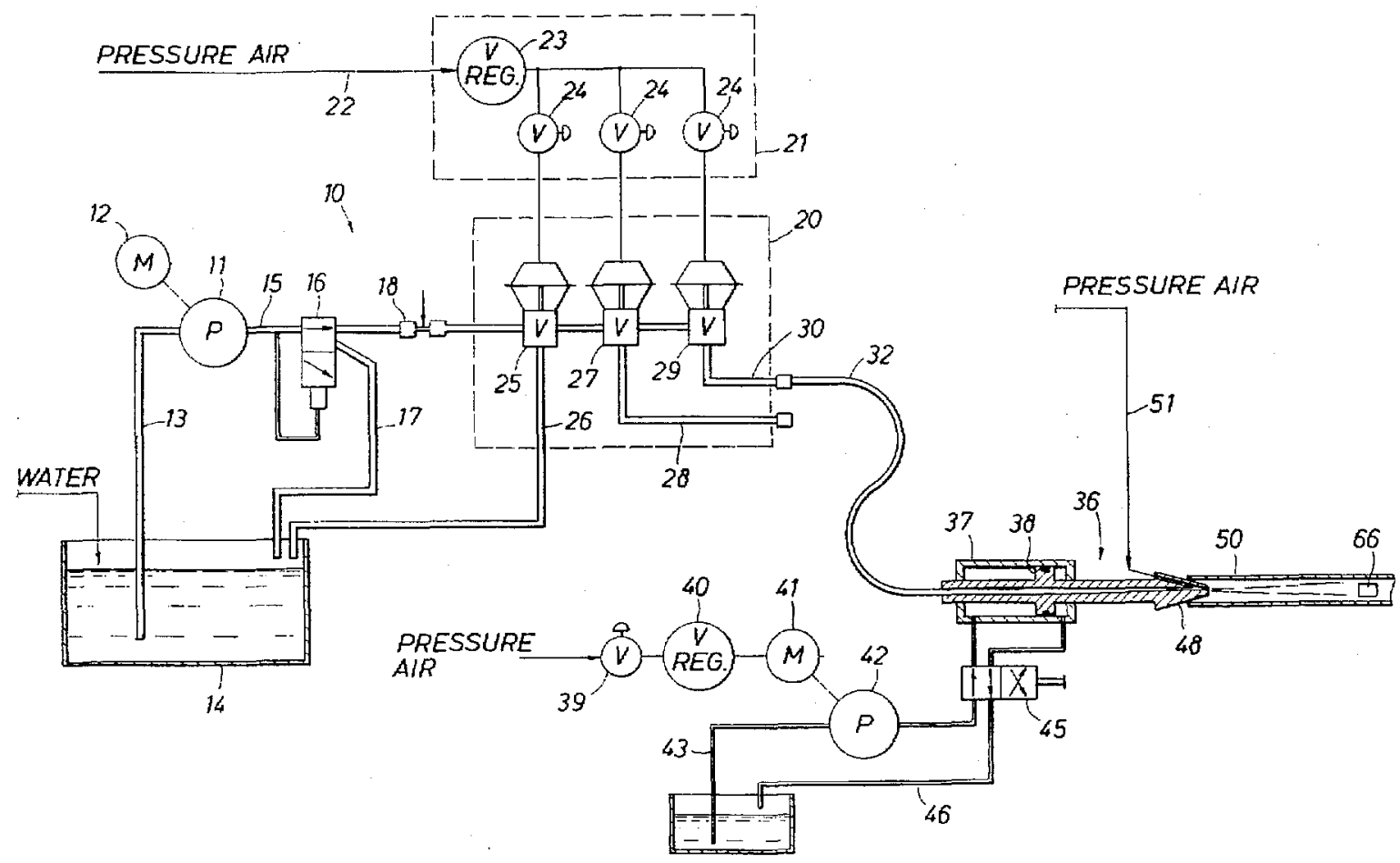




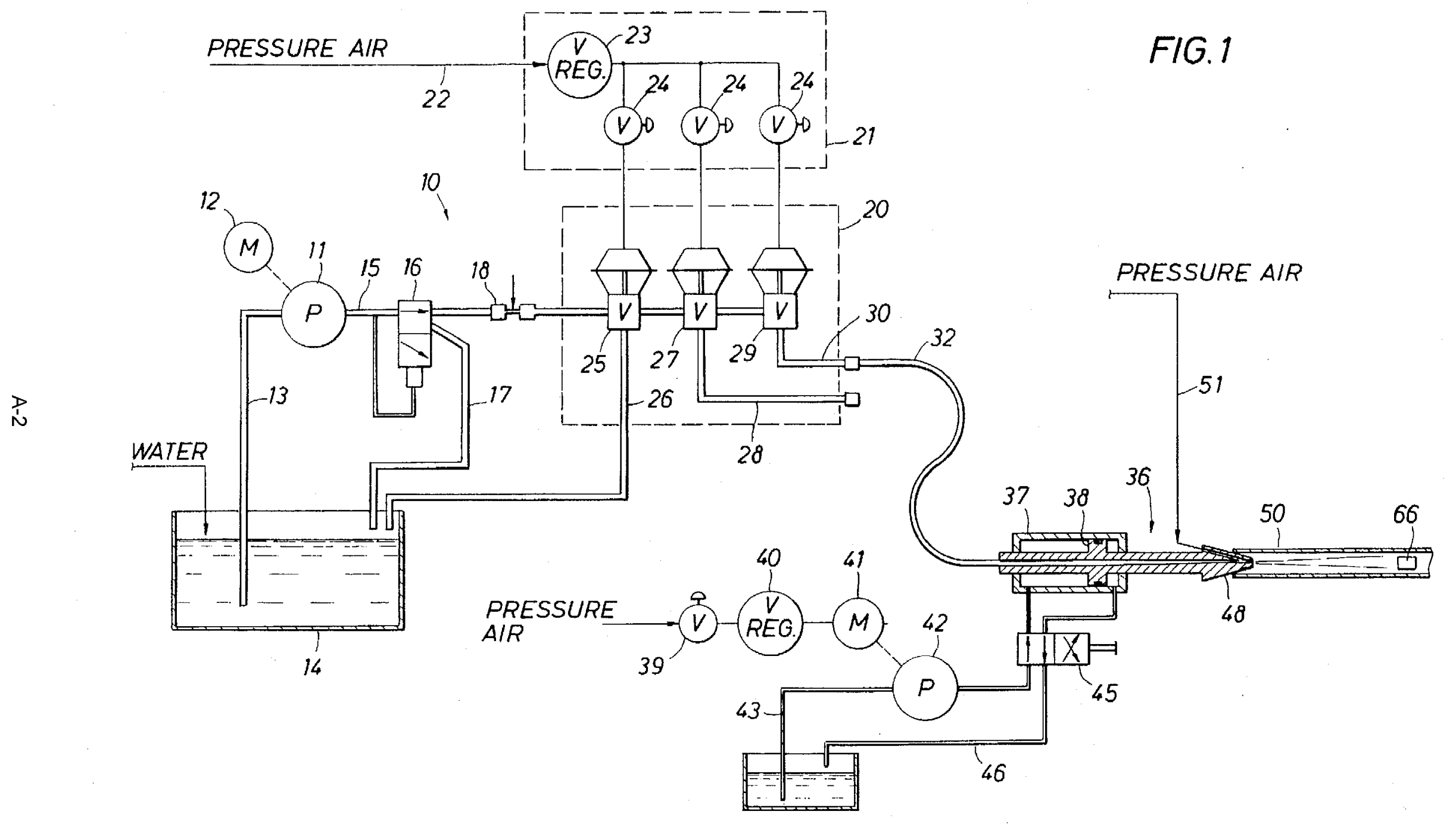

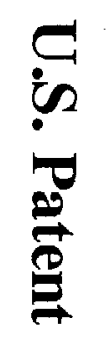

0
9
7
5

品

అָ 


$$
\begin{array}{rr}
-3 & 5 \\
& 4
\end{array}
$$

FIG. 2
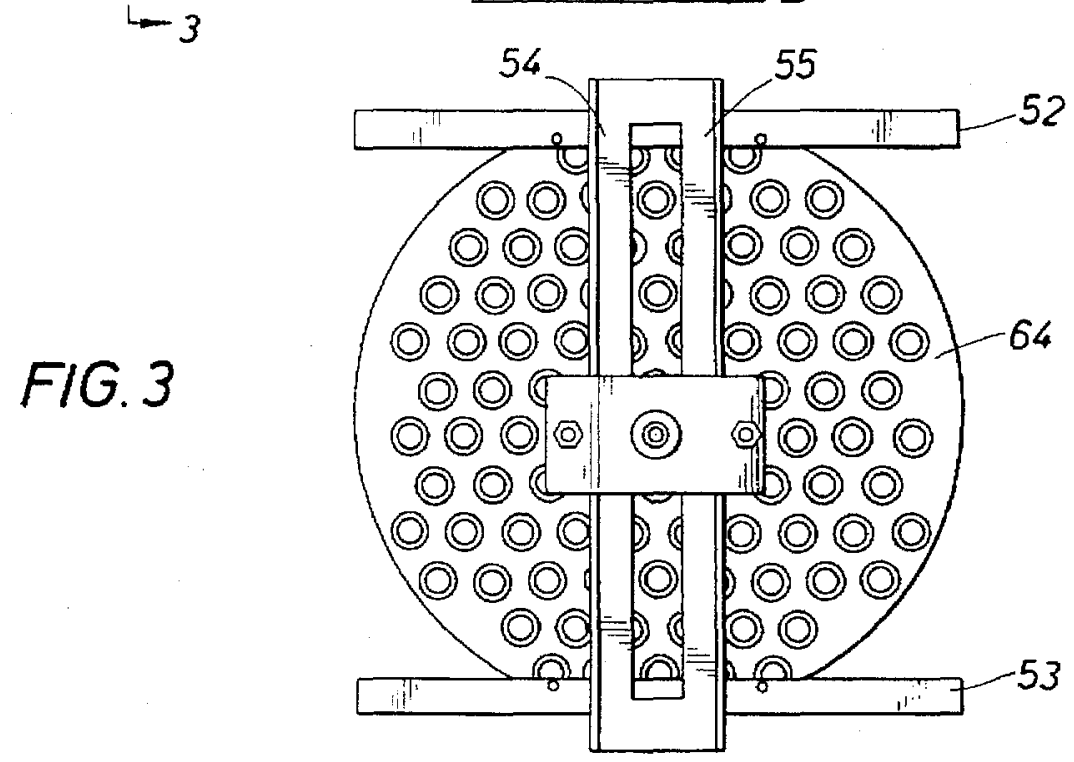

$F / G .4$

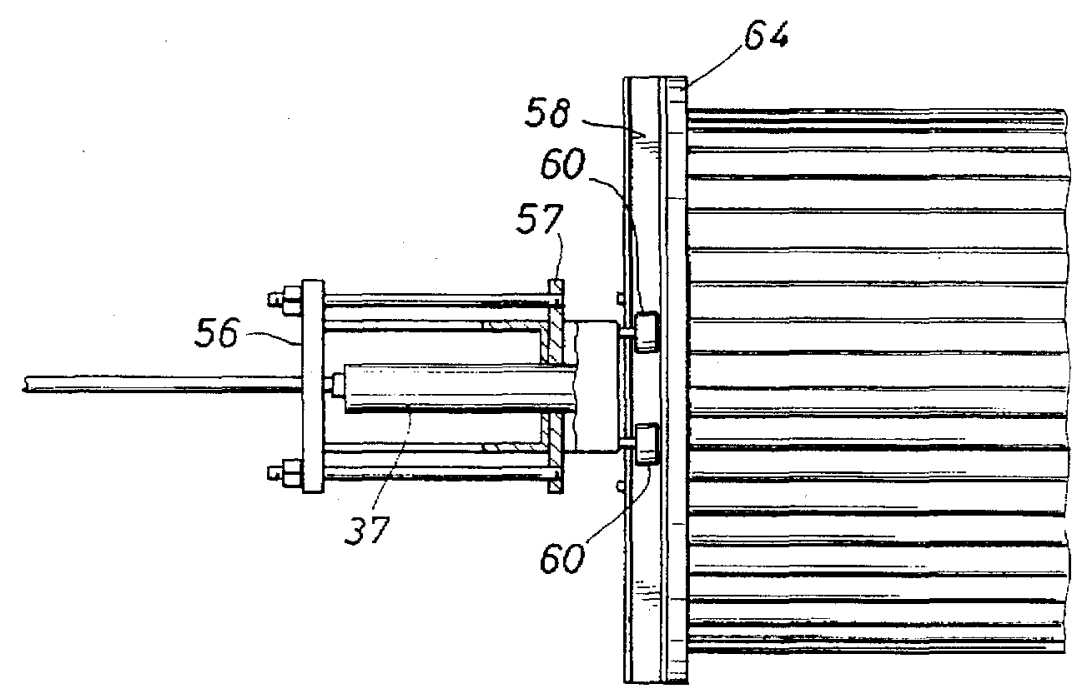




\section{1}

\section{METHOD AND APPARATUS FOR CLEANING COLUMNS BY INDUCING VIBRATIONS IN FOULING MATERIAL AND THE COLUMN}

This disclosure is a continuation in part of Ser. No. 08/016,855, filed Feb. 12, 1993, now U.S. Pat. No. 5,423, 917 issued on Jun. 13, 1995.

\section{BACKGROUND OF THE DISCLOSURE}

In virtually every conceivable industry involved in manufacturing, production or processing, fluids or gases are transported through piping, tubing, lines or other openended columns. These columns are of an infinite range of sizes (length and diameter) and made from a variety of materials. They are frequently straight, but more often than not they have corners, bends, U-turns, coils, spirals and such. Often piping or tubing is in sets or bundles. Often fluids or gases contact the exterior of the piping or tubing as well as the interior to cool or heat the fluids or gases. Sometimes the open ended column is exposed to the elements, and if not properly insulated, the fluids or gases which might be flowing within can be heated or cooled. Transportation of fluids and gases within the column is generally at a specified flow rate. Adverse results such as faulty operations or changes in the flow rates derive from faulty operations such as deposits collecting on the interior walls of the column. These deposits may be referred to as fouling material.

Occasionally, it is necessary to interrupt the process in order to clean fouling material from the interior of the column, to test the column or to perform routine maintenance. In a plant or factory environment, such activity generally takes place during annual or planned shutdown, a costly loss of production added to the actual cost of maintenance. Additionally, cleaning, testing. maintenance, etc. of the interior of the column may frequently be required when a shutdown is not scheduled but when there are signs of a need, such as when flow is impeded by an accumulation or build up of fouling material. In cases where piping or tubing is intended to cool or heat the interior or exterior fiuids, the build of fouling material can act as unwanted insulation and degrade heat transfer. Columns also logically need to be cleaned if a different medium is processed. Where a unit is permanently dismantled, the column must be cleaned when the debris within them poses any environmental concern.

Typically at maintenance time, piping and tubing is dismantled and removed from its structure, entailing costly pipefitting, crane work etc. When piping or tubing with corners, bends, flanges, valves, etc. is involved, additional work is needed to remove them, leaving only straight sections of piping or tubing, in order to ease maintenance activities. Piping or tubing that might be bundled together are commonly left bundled together, but still most bends and such are generally removed.

Maintenance, cleaning or testing can be further complicated by the fact that the piping or tubing may be long and narrow, thus not permitting easy access. Typical cleaning methods, though only somewhat successful, include brushing with wire brushes on long rods and/or drilling into the fouling material, but severe damage to the piping or tubing may result, and such methods are very time consuming and have limited effectiveness. Sometimes chemicals are circulated through the interior in an attempt to remove the fouling material, but this is only successful when flow of selected cleaning fluids is possible and is not successful in cleaning
2

blocked tubes or pipes. Probably the most commonly used method is cleaning by pumping high pressure water (hydroblasting) into the piping or tubing. Hydroblasting is also used for testing for leaks. These techniques impose

5 safety and environmental concerns and are only somewhat effective.

One prior technique uses a single valve, pressure based annular jetting system as described in U.S. Pat. No. 4,724, 007 , which is based on pushing a pig through a tube under ${ }_{10}$ high pressure. The patent states "It is now thought that the initial breakdown is not necessarily due to sonic energy and that what might have been sonic energy is more likely to be some mechanical effect akin to the effect produced in water hammer. Furthermore, at the temperatures over the time scales used, the polymer breakdown discussed by Boundy and Boyer is unlikely to occur." Pressure based annular jetting or pigging has failed at actually cleaning tubes either in the field or in public demonstrations and therefore, like brushing, drilling and hydroblasting, is also not a successful alternative. The description of annular jetting in the patent expresses many unknowns. The presence of many unknowns contribute to a system which is difficult or impossible to control.

The methodology described in the following disclosure, 25 referred to herein as hydrokinetics, has distinct differences from the annular jetting system (cleaning by pigging using water hammer shock waves), where one major difference is that the annular jetting system is based on pushing or hammering a pig through a tube while hydrokinetics induces 30 a sonic, subsonic or supersonic resonance (hereinafter called "sonics") in a tube or pipe for cleaning purposes. No pig is used with hydrokinetics, but in instances where the pipe is not completely blocked with fouling material, a blockage may be inserted in the pipe. This blockage can be anything of sufficient size and texture to close off the diameter of the column, such as paper toweling, polyethylene sheets, foam, rubber, etc. This blockage is helpful for the induction of sorties and is not pushed through the pipe. In the event of a solidly fouled pipe, no manually inserted blockage is nec40 essary since the fouling material serves as the blockage. The blockage often is blown from the pipe before or with the fouling material, unlike the pig in annular jetting which is blown along the robe behind the fouling material which the pig is pushing. Further the blockage device is proportioned to the size of the pump used. With a smaller pump, tighter clearance around the blockage is used. The sonics apparently acts on the downstream edge of the blockage where the fluid stream flows around it. With annular pigging, the effect is on the back of their pig where force is applied.

The pig used in annular jetting is relatively incompressible to be-able to push the fouling material. The blockage device used in hydrokinetics may be incompressible or not. Compressible paper toweling or wadded plastic may be used so long as it provides a blockage. No pig launcher is needed. 55 No pig catcher is needed.

Another primary difference is that annular pigging is comprised of applying a very rapid pressure increase as one end of a pig whereas hydrokinetics (as hereafter described) is based on slow filling of the pipe, and then release of high 60 velocity fluid into the relatively static fluid in the pipe. Because annular pigging involves very rapid pressure build up, the method is limited to pressures below the tensile yield of the pipe or the pipe will burst. Hydrokinetics does not have this limitation. It is believed that the pipe is not subject 65 to the actual pressure, only the created resonance.

Another primary difference is that annular pigging requires maintaining pressure on the pig for a sufficient time 
3

to force the pig completely through the tube. This hydrokinetics process is based on rapid release of fluid for only long enough to create the sonics surge into the aforementioned static fluid with no regard for forcing any projectile along the pipe.

One similarity is that both methods use a cylinder multi positive displacement pump. However with hydrokinetics it is desirable that a positive displacement pump include an odd number of cylinders. With a pulsating device downstream from the pump or fluid pressure source, any type of pump capable of producing the needed pressure can be used.

One significant difference is that annular pigging requires only a single valve to cut the fluid stream off and on. Hydrokinetics is a more involved method which uses at least two valves in addition to an unloader valve to induce resonance into the water in the pipe. This cannot be done with only a single valve.

Pigging primarily forces the pig violently into contact with the fouling material. Pig blockage devices in this hydrokinetics method does not by violent contact the fouling material. Pigging movement is so violent that water hammer and shock may occur. However, hydrokinetics induces sonics in the water of the system upstream of such blockage, at the very beginning at the pump or pulse generator operation and is enhanced by all the accelerators.

Sometimes shock from the violent contact of the pig and the fouling material in the annular pigging process may alter the contaminant material or its bond to the tube wall causing the material to change particulate or granular form. Hydrokinetics acts on contaminant material bond on the tube and the material is not changed from its particulate or granular form; rather the form is not altered. Fouling material expelled generally is large sections and has the same form prior to cleaning.

With annular pigging, successively repeating the process is desirable as layers of deposits are removed, using pigs of successively larger diameter, evidencing that the bond of the fouling material to the tube wall is not fully broken. Hydrokinetics breaks the cohesion or bond between the pipe wall and the fouling material, thus removing the entire mass of fouling material. Obviously the fluid stream may wash loose or easily removed fouling material out of the pipe.

In annular pigging, minor leakage around the is desirable. With hydrokinetics, leakage is avoided because it disrupts the laminar flow as hereinafter described.

With hydrokinetics, pigging does not take place while a blockage is manually inserted when the fouling material does not totally block the tube. This blockage device is not a scrapper. With annular pigging, the pig emerges from far end of the tube normally undamaged. With hydrokinetics, the blockage device is often distorted, probably due to the sonics and high velocities. Groves are formed along the side of the blockage devices, apparently made by scrapping the pipe wall. On close examination, it appears that the groves are back to front, negating scrapping. It is believed that these groves are the byproducts of cavitation around the blockage device.

This disclosure sets out a methodology and associated apparatus for the induction of a sonic, subsonic or supersonic resonance in the interior diameters of columns, pipes, tubes, lines, ducts, conduits, hoses, catheters, funnels and similar structures whether cylindrical or not, including stock which is square, star-shaped, round or triangular in cross section (such tubing or pipe hereinafter referred to as 65 "pipes"). The present methodology system is a valved multi velocity based sonic system, whereby sonic frequency is

\section{4}

induced along the water filled column. This sonic shock may be used to break the cohesion between fouling material and the pipe wall so the fouling material is washed away in the liquid in the pipe. This is in contrast to annular jetting 5 systems which remove successive layers of fouling materials, usually layer after layer. The disclosed hydrokinetic system. when used for cleaning, generally removes $100 \%$ of the fouling material even where it has several layers. Unlike annular pigging systems, the system is effective on any type of fouling material which will respond to the induced sonics, not just large polymeric molecules of repeating monomers or co-polymeric (involving two or more monomers) fouling material exemplified in U.S. Pat. No. 4,724,007. For instance, in a dairy, hydrokinetics can 5 remove butterfat buildup in lines, or in a plant using sea water, hydrokinetics can remove clams clinging to the interior of the lines. This is not a pressure surge water hammer system common in the pressure based annular pigging system.

20 To induce a sonic wave into a pipe via this hydrokinetics process, a pump or other high pressure fluid source, an unloader valve, fluid accelerator(s), two valves and a ram and nozzle assembly are used. A lance is not a required part of hydrokinetics. The fluid source may be smooth or 25 pulsating, for example an odd numbered, multi cylinder positive displacement pump such as a triplex pump. The fluid source can connect to a pulsation source downstream to add pulsations to the fluid flow. In the series of valves, one must be a normally closed bypass valve plumbed into the 30 system to facilitate it valve opening during bypass mode. The pipe is often entirely blocked with fouling material at some point along the length of the pipe. If the fouling material does not block the flow, a plug is placed in the pipe to emulate such a blockage.

35 In brief, hydrokinetics entails the delivery of a fluid stream from the pump or other fluid source into columns, piping, tubing, lines, ducts, conduits, hoses, reeds, catheters, funnels, and/or other open-ended columns (again, hereinafter referred to as "pipes") via apparatus which creates sound 40 waves in the fluid system and which are transferred to the bond of the fouling material. Being of different materials, the wall of the pipe and the fouling material resonant at different rates, breaking the bond between the two. Once the bond is broken, the fouling material washes out in the fluid stream. 45 Since the system is not dependent upon erosion or scrapping of the fouling material, it is likely that $100 \%$ of the fouling material will be removed.

\section{BRIEF DESCRIPTION OF THE DRAWINGS}

So that the manner in which the above recited features, advantages and objects of the present invention are attained and can be understood in detail, more particular description of the invention, briefly summarized above, may be had by reference to the embodiments thereof which are illustrated in 5 the appended drawings.

It is to be noted, however, that the appended drawings illustrate only typical embodiments of this invention and are therefore not to be considered limiting of its scope, for the invention may admit to other equally effective embodi60 ments.

FIG. 1 is a schematic flow diagram of the system forming a shock wave as set forth in the present disclosure and shows in a combined schematic the fluid flow of air and water in the system;

FIG. 2 is a side view of a lance mounting mechanism showing a lance which extends to seat against a tube to enable tube cleaning; 


\section{$5,674,323$}

\section{5}

FIG. 3 is a sectional view along the line $3-3$ of FIG. 2 and shows details to construction of the mechanism which aligns the lance with a particular tube for cleaning; and

FIG. 4 is a sectional view along the line $4-4$ of FIG. 2 showing details of construction of the lance insertion mechanism.

\section{DETAILED DESCRIPTION OF.THE PREFERRED EMBODIMENT}

According to the article, "The Chemical Effects of Ultrasound", Scientific American, February 1989, large bubbles or cavities, imploded by water pressure are a source of vibrations. The process will be herein referred to as "cavitation". Intense ultrasound waves generate large alternating stresses within a liquid by creating regions of positive pressure and negative pressure; a cavity can form and grow during the episodes of negative pressure but when the cavity attains a critical size, the cavity implodes, generating intense heat and tremendous pressure. More specifically, the article suggests that vibrations are due to the tremendous turbulence, heat and pressure of the imploding cavities, providing a unique environment for high energy reactions. Cavitation can be induced by generating intense sound waves in a liquid. Such waves create alternating regions of compression and expansion that can form bubbles subject to implosion. Of course, compression cycles exert a pressure on the liquid molecules forcing them together. Conversely, expansion cycles exert a negative pressure which pulls the molecules away from one another. According to the article, "During the expansion cycle a sound wave of sufficient intensity can generate cavities."

A liquid is held together by attractive forces, which determine surface tension of a liquid. For a cavity to form, a large negative pressure associated with the expansion cycle of the sound wave overcomes the liquid tensile strength. The article explains that less pure liquids have weaker tensile strengths. Thus the induction of soda or a metalliferous medium, as described hereafter, enhances the formation of cavities. Further, the adhesive nature of a liquid is cut when the liquid is gas cut, or gas is dissolved in the liquid. "When a gas-filled crevice is exposed to a negativepressure cycles from a sound wave, the reduced pressure makes the gas in the crevice expand until a bubble is released into solution. Most liquids, such as tap water, are sufficiently contaminated by small particles to initiate cavitation."

According to the article, bubbles in liquid are inherently unstable (large ones tend to float to the surface and small ones tend to redissolve into the liquid), but bubbles absorb energy with the compression and expansion cycles of sonic waves. "The growing cavity can eventually reach a critical size where it will most efficiently absorb energy from the ultrasound. The critical size depends on the frequency of the ultrasound wave. Once a cavity can no longer absorb energy efficiently from the sound waves, it can no longer sustain itself and the liquid rushes in and the cavity implodes."

During implosion, the gases and vapors inside the cavity are compressed, generating intense heat that raises the temperature of the surrounding liquid, creating a very small local hot spot which dissipates quickly. However, at any given time, the temperature of the bulk of the liquid remains unaffected.

Further according to "The Chemical Effects of Ultrasound", Scientific American, February 1989, if the cavity forms near an extended solid surface, such as the surface of the fouling material or pipe wall, the implosion
6

will be asymmetric, expelling a jet of liquid at roughly 400 kilometers per hour directed at the surface, as the jet develops opposite the solid surface and moves toward it. The jet, as well as the waves from the cavity implosion, erode 5 solid surfaces, remove non-reactive coating and fragment brittle powders. Reactions are further facilitated by high temperatures and pressure associated with cavity implosion near the surface. Thus it is believed that in the hydrokinetic system (as described in detail in the following paragraphs), in conjunction with the resonance of the fouling material and pipe wall at different frequencies, degradation of the fouling material and deep cleaning of the pipe wall surface is further impacted by the bombardment of high-speed jets of heat and energy for the imploding cavities. Hydrokinetics is effective at clearing fouling material from pits in the pipe wall, an effect very important during pipe testing.

FIG. 1 of the drawings illustrates the schematic of the system having a pump 11 which is driven by a suitable motor 12. It is provided with a feed line $\mathbf{1 3}$ from a water sump 14.

The pump 11 has a pump output 15 which is provided to a control valve 16. The valve 16 is a two position valve. In the illustrated position, water under pressure is delivered from the pump through an adjustable orifice 18. The valve 16 also connects with a line 17 which provides a return to the sump. The orifice 18 provides an input to a control cabinet 20 represented in dotted line for operator control.

The control cabinet has an air pressure manifold 21. There is a supply of pressurized air on a line 22 which is input to a regulator valve 23 . That provides a regulated air pressure 30 output through several control valves at 24 . The several regulators are input to water control valves in the cabinet 20 . The first yalve 25 is connected with a line 26 which provides another return to the sump. The valve 25 , when operated, delivers the output flow through a control valve 27 . It 35 connects with a flow line 28 for purposes to be described. In addition, flow is delivered to a valve 29 which provides an output flow that is switched when the valve 29 is operated. This output is on a line $\mathbf{3 0}$. The cabinet $\mathbf{2 0}$ has appropriate fittings on it to enable connection of a lance feed line 32 . The line 32 extends some distance, typically from 10 to 50 feet.

The lance 36 is coaxial with an elongate cylinder 37 which encloses a piston 38 . The piston 38 enables positive insertion and retraction of the lance. The hydraulic system thus utilizes air from a suitable air pressure source delivered 45 through a control valve 39 which connects to an air pressure regulator 40. An air motor 41 operates a hydraulic pump $\mathbf{4 2}$. There is an inlet line $\mathbf{4 3}$ connected to hydraulic oil sump 44 . Hydraulic oil is delivered to a control valve $\mathbf{4 5}$ to control the movement of the lance. The return line $\mathbf{4 6}$ returns the low 50 pressure oil to the sump. The lance has an elongate rod portion which terminates at a tip 48. An air inlet line 51 connects with the lanced tip $\mathbf{4 8}$ to introduce air along with the liquid.

The lance $\mathbf{4 8}$ is moved with respect to a set of tubes in a 55 fashion shown in FIGS. 2, 3 and 4. FIG. 2 shows the lance 36 which is supported and aligned by cylinder 37 . It is mounted so that it travels on a pair of parallel rails 52 and 53 shown in FIG. 3 of the drawings for movement in the X direction. A bracket is comprised of left and right frame 60 members 54 and 55. They move as a unit. They enable vertical movement of the cylinder 37 . The frame members 54 and 55 define a gap where the lance extends through the gap. The cylinder $\mathbf{3 7}$ is anchored to the spaced plates $\mathbf{5 6}$ and 57 which capture the cylinder. The guide surfaces are 65 formed along the edges of the frame members 54 and 55 and thus define the channel 58 shown in FIG. 4 for movement. Rollers 60 are located in this channel. 


\section{7}

The cylinder 37 is ,guided by the rollers 60 which clamp on the outside of the parallel frame members 54 and 55. In cleaning the tubes, the device 66 is first placed in a tube and the lance is moved in an $\mathrm{X}$ and $\mathrm{Y}$ coordinate system until it is aligned with the particular tube.

To initiate this process, any source of fluid to be fed into the system can be used so long as it is sufficient to supply the quantifies needed. Such source might be municipal fire water, plant or factory water or a portable tank containing a liquid chemical appropriate for the need. Such fluid may be pumped continuously or as needed into the holding tank for the pump, which is a pump of any type that delivers the fluid in pulsations, such as a positive displacement pump, rather than flow in a steady stream, such as a centrifugal pump. The pump is sized as close as possible to the maximum flow rate allowed for a given pipe, generally measured in gallons per minute (gpm). With the addition of a pulsating device downstream from the pump or fluid source, any type of pump capable of producing the needed pressure range can be used or, if the fluid source itself is capable of producing the needed pressure range, no pump at all is needed.

Operating pressures for hydrokinetic action range up to 20,000 psi with the typical range being about 250 to 3000 psi. This system is not a pressure intensive system. Rather the pressure serves key purposes (1) to move the resonating fluid system, (2) to wash the fouling material from the pipe once the cohesion between the pipe wall and the fouling material has been broken, or (3) to test pipe.

The fluid stream travels from the pump or pulse generator to an unloader valve, which is a very precise, adjustable, fast acting pressure relief device. When a defined pressure is exceeded at this valve, it dumps enough fluid to drop the pressure of the stream down to a targeted pressure. This unloader valve $\mathbf{1 6}$ is constantly regulating the pressure in a rapidly pulsing fashion to maintain this given pressure profile. The unloader valve increases the pulses produced by the pulsation type pump or device. This increase in pulsations can be calculated for precise control of the system but calculations are not required for effectiveness.

From the unloader valve the fluid stream is routed to a fluid accelerator, which is usually the first of two or more fluid accelerators, and then routed to the next accelerators are appropriate for the project, on to more accelerator(s). It is possible that only one high quality accelerator will achieve the velocity needed for sonic cleaning, but more than one is usually needed to reach the necessary velocity. One simple accelerator is the orifice $\mathbf{1 8}$ to increase the flow velocity. The purpose of the accelerator(s) is to increase the velocity of the fluid stream beyond the velocity normally generated by the pump or fluid source.

The fluid flows through hoses or piping to a safety control cabinet containing two or more valves. Unlike annular jetting systems which rely on one valve to simply cut the fluid stream off and on, this hydrokinetics process uses this multi valve set up to create the resonance into the "unit" (a "unit" is one pipe, a set of pipes or an entire bundles or network of pipes). These valves are (1) the bypass valve, (2) the line-out valve for activity involving one unit and (3) any number of additional line-out valves for activity involving multiple units. When open, the bypass valve, which is normally closed, routes the fluid stream to a drain or holding tank, or when a pump is used, will re-route the fluid back to the holding tank as the pump. It is recommended for safety but not necessary for functionality that all valves be spring loaded and configured in such a way as to always go into 65 bypass mode in the event that air pressure is lost or if operator intervention is lost.

\section{8}

The safety control cabinet is best as one enclosure which contains the bypass valve and line-out valves as well as the necessary gauges and controls, or can mean an enclosure for the valving with a separate enclosures for the gauges and/or 5 controls. The enclosure or walls of the safety control cabinet can be 316 stainless steel or other appropriate material designed and constructed in such a manner as to form a safe, preferably explosion-proof enclosure that will contain and disperse the pressures generated in the valve oscillator in the event of failure, disconnected couplings, etc. This enclosure can also serve as a NEMA (electrically safe) enclosure if it is preferred that the components of the system be controlled by electric power. Enclosure weight is reduced by omitting the frame, and the walls of the enclosure form its own frame, thus allowing mounting the components to the cabinet.

The suggested monitoring panel of the safety control cabinet has a high pressure output gauge, a hydraulic pressure gauge, an air pressure gauge and other appropriate instrumentation. These in some instances are also enhanced by LED signals showing the position of the pilot valves (defined hereafter).

The operation of the hydraulic systems in the safety control cabinet is controlled by a set of two-way pneumatic pilot valves. The pilot valves 24 are energized by high ${ }_{25}$ pressure air from a source of at least about $100 \mathrm{psi}$. In the event 100 psi air is not available at a given location, an additional component of the system. known as an air-to-air intensifier, is utilized to bring air pressure to a selected level. In the event that air pressure exceeds $140 \mathrm{psi}$, a regulator is utilized to bring the air pressure down to the specified air pressure.

Control levers on the safety control cabinet actuate the pilot valves. The pilot valves actuate the bypass and line-out valves. The inlet sides of the pilot valves are connected, 35 usually by high pressure hoses and fittings, to an air manifold attached to the regulator, if required, which is attached to the safety control cabinet which is attached to the air source. The outlet sides of the pilot valves are connected to a diaphragm actuator which activates the bypass and line-out 40 valves. When energized, air is directed from the air source to the top of the diaphragm, which pushes down upon a plunger, which activates the bypass valve and the line-out valve. When in the de-energized position, air that was used to push against the diaphragm is allowed to flow back 5 through the connecting hose and is exhausted via a port in the pilot valve to an exhaust outlet located in the side of the safety control cabinet.

Within the safety control cabinet, there is a high pressure fluid oscillator block and valves. The oscillator block is 50 constructed of a material such as carpenter grade high tensile stainless steel or high alloy steels (for use with highly chlorinated water as a fluid stream). This oscillator might be cylindrical in cross section and should have a wall thickness sufficient to handle triple the maximum pressure from the 55 pump or fluid source. This oscillator block is mounted to the cabinet to allow it to vibrate freely.

The bypass valve and the line-out yalves are poppet valves. They are actuated by the pneumatic actuators described above. The inlet side of the oscillator is connected 60 to the system via hoses or pipes. The outlet side of the oscillator is two phase. In the bypass mode, when the line-out valve is closed and the bypass valve is open, the bypass valve allows fluid to circulate through the oscillator at low pressure and back to the holding tank or drain. A 65 heavy wall high pressure pipe can be attached to the bypass outlet so that additional vibrations or harmonics can be induced in the system by adjusting the length of this pipe. 
9

Staging mode is the mode of the procedure that prepares or stages the system for resonance into the fouled pipe or pipe to be tested The line-out valve and the bypass valye are both open, allowing low pressure fluid to fill the pipe up to the point where the pipe is blocked with fouling material. In the event the fouling material only partially blocks the flow of fluid, a blockage device is added in the pipe. This blockage may simply be a wad of paper, plastic, foam or other such object, and it is often a plastic or brass plug, which appears at first glance to be a pig as used in the annular jetting method. However, its purpose is to act as a plug, not to be driven through the pipe as the cleaning device. There is not a rapid inflow of fluid as in the annular jetting system, but simply a filling of the pipe. The blockage of fouling material collects debris which washes out in the fluid stream under the pressure inherent to the system, leaving only a partial blockage, or plug which must be placed in the pipe.

Operational mode is the mode of the procedure in which the pulsations are transferred via the fluid stream (which has already filled the pipe) via a nozzle (the nozzle is described 20 hereafter) to the pipe. In this mode the bypass valve is closed. Because this valve is closed and because the pipe is blocked either by fouling material or a plug, and thus no fluid is allowed to escape anywhere in the system, pressure builds throughout the entire system, from the fluid source forward all the way to the blockage. The line-out valve is still open in the operational mode. As pressure builds in the oscillator (as described above) of the cabinet, the oscillator and the fluid within will begin to vibrate. This mode may only last a fraction of a second, after which the bypass valve is reopened.

There is of course a frequency at which the pipe, dependent upon its composition, will begin to vibrate and a frequency at which the fouling material, dependent upon its composition, will begin to vibrate. Composition for these purposes is as size, thickness, density, support structure and other criteria which control the frequency at which an item will vibrate. Because the pipe and the fouling material are of different compositions, they will almost always vibrate at different frequencies, except in the rare instance where both the pipe and the fouling material vibrate at the same frequency. When the frequency at which each will vibrate is met in the fluid stream by the build up of pressure in the pulsations, from the pump or pulse generator, this frequency will result in the vibration of the pipe and separately in the vibration of the fouling material. This separate vibration results in a breaking of the bond between the pipe wall and the fouling material. Once this cohesion is broken, the blockage which inhibited the flow of the fluid stream will wash forward under the pressure inherent to the system and the blockage and other fouling material will wash out of the pipe. Then, it is extremely easy to remove fouling material, debris is washed out with a simple garden hose, or which is washed under the pressure inherent to the system.

This system cleans when the necessary frequency range does not exceed an augmented frequency range, such as the frequency range arrived at from the 120 degree pulsation of a triplex pump when the pump rotating at approximately 450 $\mathrm{rpm}$ and modulated through the unloader system at a pressure low enough to avoid structural damage to the fouled pipe. In the event that the frequency range needed to induce a resonance in the pipe exceeds the normal operating capabilities of the hydrokinetic system, the frequencies can be raised further via manipulation of the bypass and line-out valves, in the following manner.

The line-out valve is closed after the pipe is filled with fluid. Bypass valve is closed. Pressure is allowed to build in

\section{0}

the oscillator block. This pressure is modulated into the already filled tube via manipulation (rapid off and on) of the line-out valve. Much higher pressures and higher frequency ranges can be achieved and transferred to the pipe wall without causing structural damages via sympathetic vibration. In the event that still higher modulation might be required, this is achieved in some instances by the insertion of a vibrating reed into a holder affixed at the inlet side of the high pressure oscillator and/or the aforementioned vibrating reed is attached to the outlet side of the bypass valve. Fluid moving at a high velocity across the top of the reeds causes the reeds to vibrate. The thickness and length of the reeds determines their vibrational frequencies. Another method of achieving the same effect is the utilization of an eccentric cam rotated by a motor.

Additionally, sound frequencies can be fed into a static stream via a tone generator or oscillator. This oscillator can also be automated. A computer program can instruct the tone generator to give out a modulating frequency with a preset low frequency and high frequency range. The high and low frequencies are determined by attributes of the pipe (such as the material of construction, length, diameter, and wall thickness) and attributes of the fouling material. As the tone generator sweeps between the preset low and high frequencies, a standard frequency analyzer mounted on the back of the pipe will pick up and lock onto the actual frequency at which the pipe will resonant. The information can be fed back to the computer and the computer can lock the tone generator onto this frequency, allowing resonance 30 of the pipe without regard to the pressure generated by the pump or fluid source.

When the system is in the Operational mode, the fiuid stream travels, as described above, through the line-out valve to the pipe via tubing or hoses. At the face of the pipe, 35 to inject the fluid stream into the pipe, a ram and nozzle assembly is used. The ram is mounted at the face of the pipe or set or bundle of pipes to allow hydraulic, electronic or manual movement of the nozzle in and out of each tube. It is recommended for safety but not required for functionality 40 that a check valve be plumbed in the hydraulic line, so that once the ram is energized with the nozzle against the face of the pipe, if hydraulic pressure is lost, the ram and nozzle assembly will not come away from the pipe face until such check value is manually tripped. The ram is coaxial (moves forward and backward) with an elongate cylinder which enclosed a piston. It is unique in that it has a tapered bore and the fluid runs through the piston rod, eliminating the need for additional pipe firings. The taper of the bore is such that the orifice at the outlet of the ram is approximately 15 to $20 \%$ 50 smaller than the orifice at the inlet of the ram. As a safety mechanism, at the inlet side of the ram, a machined bell nipple connects to a coupling on the hose to the ram. In the event of any type of failure of this coupling, this bell shape acts as a diffuser to remove the energy from the fluid stream 55 to protect personnel.

The nozzle is tapered with the outlet end, usually smaller than the inlet end of the pipe. In cases where pipes are "rolled" into pipe or tube face sheets, the taper on the nozzle preferably is the same as the taper used on the rolling tool 60 which rolled the end of the pipe onto the face sheet; thus the nozzle will reinforce this roll rather than doing damage to it. When the nozzle is inserted into the pipe and hydraulic pressure is applied yia the ram, a tight metal-to-metal seal is formed between the nozzle and the pipe. This is in contrast 65 to the desired leak needed in an annular jetting system.

Between the ram and nozzle, a nozzle adapter can be inserted. The nozzle adapter is a measured orifice machined 
11

to avoid protrusions into the fluid stream where it attaches to the ram and to the nozzle, so that the flow is laminar. The bore in the nozzle adapter is the same diameter as the bore at the outlet end of the ram, thus not increasing or decreasing the velocity of the fluid stream. Onto this nozzle adapter, a heavy duty, thickwalled, highpressure pipe can be attached. The purpose of this pipe is to add length in order to induce more harmonics into the fluid stream. If the pipe is longer, the vibration is greater.

To support the ram, nozzle adapter and nozzle, against a bundle of pipes, an X-Y alignment system can be used. This is a device such as used in laser burning, machining, cutting, etc. The X-Y axis can be freestanding or mounted to the face of the pipe bundle. The ram and nozzle assembly are moved along the $\mathrm{X}$ or $\mathrm{Y}$ axis manually or a computer automatically moves the ram and nozzle assembly along the $\mathrm{X}$ or $\mathrm{Y}$ axis upon command. This requires programming on mechanisms such as those used to move lathes, mills, drill presses, etc. This would facilitate the use of Hydrokinetics in environments where manual movement would be difficult or prohibitive, such as in nuclear waste processes.

It is an important, but not necessarily an essential part of the hydrokinetic system, that the flow of fluid, from the pump or fluid source to the upstream side of the nozzle, be as streamlined as possible. Protrusions into the fluid system, such as a bolt protruding through the line into the fluid stream, or by high friction internal linings of the pipes are avoided. The flow of the fluid stream is as "laminar" as possible. This is opposed to a "boundary layer flow" in which the outer portion of the radius of the stream is slowed by frictional drag and flows at a slower velocity that the inner portion of the stream, or turbulent flow.

The fluid stream changes from laminar flow to boundary layer flow at the outlet nozzle tip. In the pipe to be cleaned, filled with static fluid from the nozzle tip, a pulsating fluid stream pumped into the center of the pipe, sets up a reflected shock wave and resultant standing wave in the column of water. The standing wave frequency will pass through the resonance frequencies of the fouling material. The fluid is thought to collapse bubbles during the low pressure pulse resulting in cavitation. In addition to the breaking of the cohesion between the pipe wall and the fouling material, loose or easily removed fouling material simply washes out in the fluid stream while the cavitation breaks the fouling material bond which becomes loose and washes free.

To enhance the boundary layer flow at the outlet of the nozzle tip, a high pressure air manifold connected to the nozzle adds measured pulses of gas to the fluid stream, enhancing the cavitating effect.

In some situations, it may be faster and provide a higher. degree of cleanliness or polishing, to add soft abrasives, such as sodium bicarbonate or polymers, to the fluid stream. Soft abrasives and other mediums can be added wet or dry. Dry materials, such as various bicarbonates, are injected at the nozzle into the static fluid used to fill the pipe during the Staging ,mode or into the resonating fluid stream during Operational mode. Upstream of the first accelerator, part of the fluid stream from the pump or fluid source can be mixed with the medium to be injected. The abrasive material is blended with part of the fluid stream and the solution is added into the main fluid stream downstream. The controls needed for dry or liquid medium injection are pneumatic metering valves. A liquid surfactant or cleansing agent can be added.

According to the article, "The Chemical Effects of Ultrasound", solid particles, such as soda or the metallic

\section{2}

elements in a metalliferous medium, are heavily impacted by the effect of waves caused by cavitation of bubbles when sonics are induced in a liquid. (discussed in more detail hereafter). Apparently, the pressure waves drive small par5 ticles into one another at high speeds with collisions so intense that the metal powders are melted at the point of impact. This melting can remove metallic-oxide coatings, which protect the metals. Concluding, the article says "Ultrasound can increase the reactivity of metal powders by 10 more than 100,000 time", which appears to cause metal particles to bond. Thus the addition of soda or chemicals of a metallic nature can enhance the cleaning.

The intensity of cavity implosion can easily be altered by changing frequency, acoustic intensity, temperature, static 5 pressure, choice of liquid and choice of gas. In the article, implosion proceeds more slowly as ambient temperature increases so the fluid stream can be cooled to enhance cleaning. The fluid stream can be warmed to reduce cleaning.

Tests have shown hydrokinetics to be particularly effective for removing hydrocarbon based deposits. According to the article, organic compounds are highly degraded in this environment, and inorganic compounds can be oxidized or reduced.

While the foregoing is directed to the preferred embodiment, the scope thereof is determined by the claims which follow:

What is claimed is:

1. A method for cleaning a fouled pipe comprising the steps of:

(a) filling the pipe with an incompressible liquid;

(b) releaseably connecting to the pipe a pressure source;

(c) by means of cooperating valves, pulsating the pressure source to form a standing wave in the liquid in the pipe;

(d) by means of said valves, adjusting said pulsations so that cavitation occurs in the liquid during pressure fluctuations within the standing wave; and

(e) by means of said valves, adjusting the pressure of the pulsations such that said cavitation creates vibrations of differing frequencies transmitted through the liquid to the fouling material and to the pipe, wherein the fouling material and the pipe vibrate at different frequencies to break the fouling material free of the pipe.

2. The method of claim 1 wherein the pipe is constructed with an inlet end and an outlet end, and the pressure pulsations are delivered into the pipe at the inlet end.

3. The method of claim 2 wherein the outlet end of the pipe is plugged by a blocking device, and wherein the 50 blocking device is temporarily fixed to the pipe and retains the liquid within the pipe.

4. The method of claim 3 wherein the blocking device temporarily holds against specified pressure levels thereby allowing the standing wave to be formed, and is expelled 55 from the pipe on increase above the specified pressure levels.

5. The method of claim 4 wherein the blocking device is a sacrificial insert initially positioned in the pipe and is blown from the pipe on increase of pressure after cleaning 60 to thereby enable the pipe to be cleared of the liquid which flows out of the pipe and carries dislodged fouling material with the liquid.

6. The method of claim 5 wherein the inlet end is provided with the pressure pulsations by fitting a nozzle at the inlet 65 end.

7. The method of claim 6 wherein the inlet end is provided with two inlet lines, and one of the inlet lines delivers 


\section{$5,674,323$}

\section{3}

flowing gas admitted to the pipe, and the other of the inlet lines delivers the incompressible liquid.

8. The method of claim 5 including the step of forming bubbles in the liquid subject to imploding during pressure fluctuations.

9. The method of claim 8 wherein bubbles are collapsed and reformed.

10. A method of cleaning an interior of a fouled pipe comprising the steps of:

(a) in a region of the pipe where the fouling occurs, isolating a portion of the pipe between an inlet end and an outlet end;

(b) filling the pipe between the inlet end and outlet end with an incompressible liquid; and

(c) forming microscopic bubbles within the liquid by generating standing waves in the liquid;

(d) applying pressure yariations to the liquid so that the bubbles in the liquid are momentarily compressed and released therefrom to thereby initiate collapse of the bubbles to generate vibrations in the liquid for breaking the bond holding the fouling material to the pipe, wherein

(i) the vibrations are generated at multiple frequencies,

(ii) the frequencies of the vibrations are controlled by the magnitude of the pressure variations, and

\section{4}

(iii) the bond is broken by vibrating the fouling material and the pipe at different frequencies.

11. The method of claim $\mathbf{1 0}$ including the step of mixing a cleaning abrasive in the liquid prior to applying the 5 pressure variations to the liquid, wherein the interior of the pipe is further cleaned by the cleaning abrasive after the fouling material bond has been broken and fouling material has been removed.

12. The method of claim 10 including the step of mixing a compressible gas with the liquid.

13. The method of claim 10 including the step of controlling temperature of the liquid to thereby control an extent of cleaning in the pipe.

14. The method of claim 10 including the step of blocking the outlet end of the pipe with a pressure controlled blocking

15 device and the blocking device is left fixed in the pipe during cleaning so that liquid and the blocking device are forced by increase of pressure from the pipe at conclusion of the cleaning.

15. The method of claim 14 including the step of pumping 20 added liquid into the pipe after filling with pressure fluctuations.

16. The method of claim 15 further including the step of pumping the added liquid with time dependent pressure fluctuations. 
(12) United States Patent Garcia, Jr.
(10) Patent No.: $\quad$ US 7,421,757 B1

(45) Date of Patent:
(54) PUMP VALVE MECHANISM

(75) Inventor: Ralph Garcia, Jr, Houston, TX (US)

(73) Assignee: AIMM Technologies, Inc., Texas City, TX (US)

(*) Notice: Subject to any disclaimer, the term of this patent is extended or adjusted under 35 U.S.C. 154 (b) by 781 days.

(21) Appl. No.: 10/920,079

(22) Filed: Aug. 17, 2004

(51) Int. Cl. BOSB $9 / 43 \quad(2006.01)$

(52) U.S. Cl. $15 / 304 ; 15 / 302 ; 15 / 321$; $134 / 1 ; 134 / 167 \mathrm{C}$

(58) Field of Classification Search ............... 15/304, $15 / 302,301,321 ; 134 / 167 \mathrm{C}, 184,169 \mathrm{C}$

$134 / 1$

See application file for complete search history.

References Cited

U.S. PATENT DOCUMENTS

$5,423.917 \mathrm{~A} * 6 / 1995$ Garcia, Jr
$5,493,754 \mathrm{~A} * 2 / 1996$ Gurstein et al. ............. 15/321

$5.647,089 \mathrm{~A} * \quad 7 / 1997$ Hollrock ...................... 15/302

$5,674,323$ A * 10/1997 Garcia ...................... 134/1

6,282,746 B1* 9/2001 Schleeter .................. 15/302

$7.141,118$ B2 * 11/2006 Vaerewyck et al. .......... 118/50

$7,306,001 \mathrm{~B} 1 * 12 / 2007$ Garcia, Jr $134 / 167 \mathrm{C}$

* cited by examiner

Primary Examiner-Lee D Wilson

(74) Attorney, Agent, or Firm -Law Office of Tim Cook P.C.

\section{ABSTRACT}

A method for cleaning tubes and heat exchangers provides an oscillating pump/valve mechanism to provide a periodic waveform of shocks to fouled tubes. Preferably, a pair of pneumatic control valves operates an actuator to oscillate a valve within a valve block, and isolation of a fluid within a manifold in the block creates a pumping action from the valve, to create an intense fluid shock wave to clean interior surfaces tubes.

4 Claims, 3 Drawing Sheets

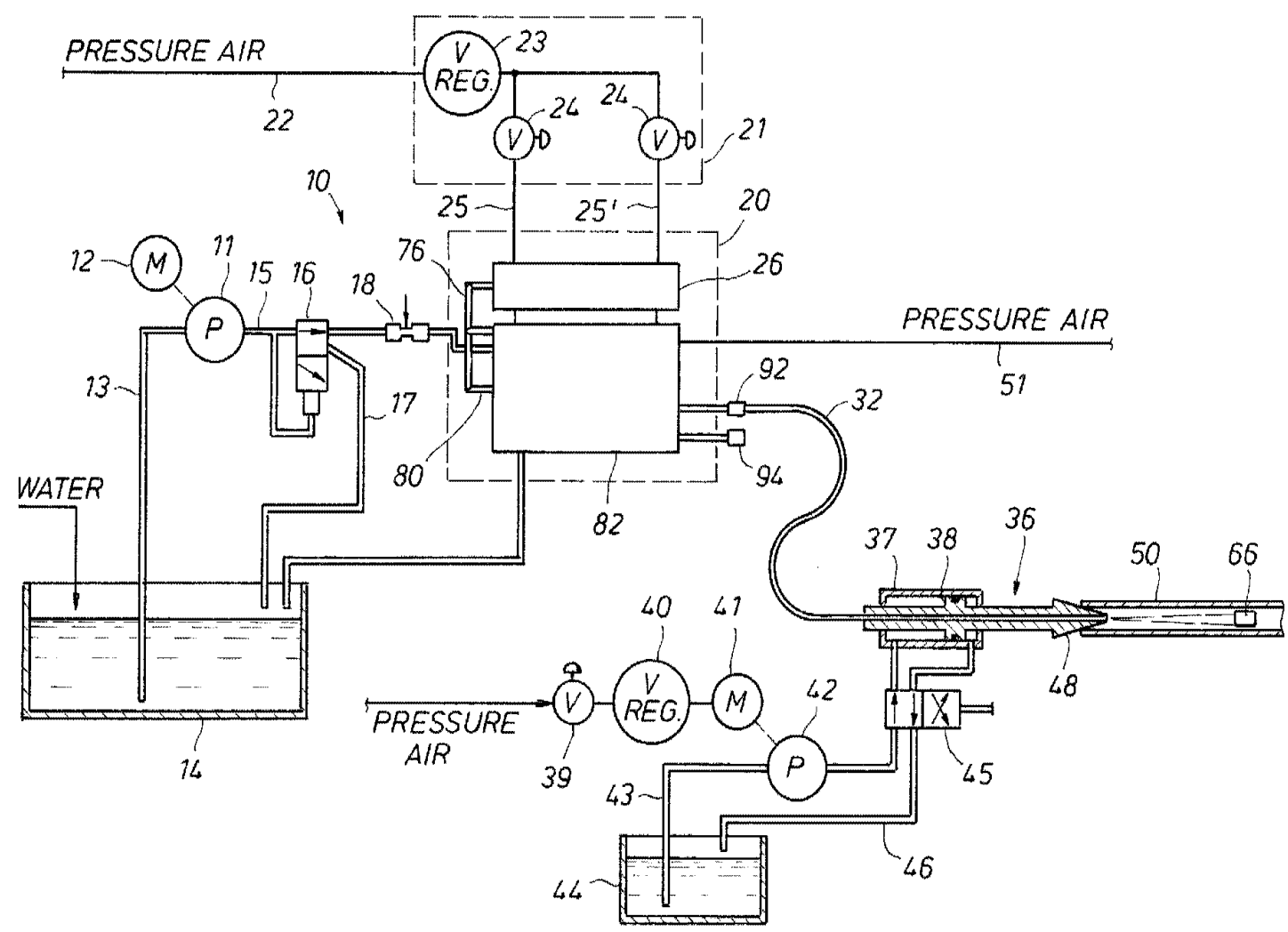




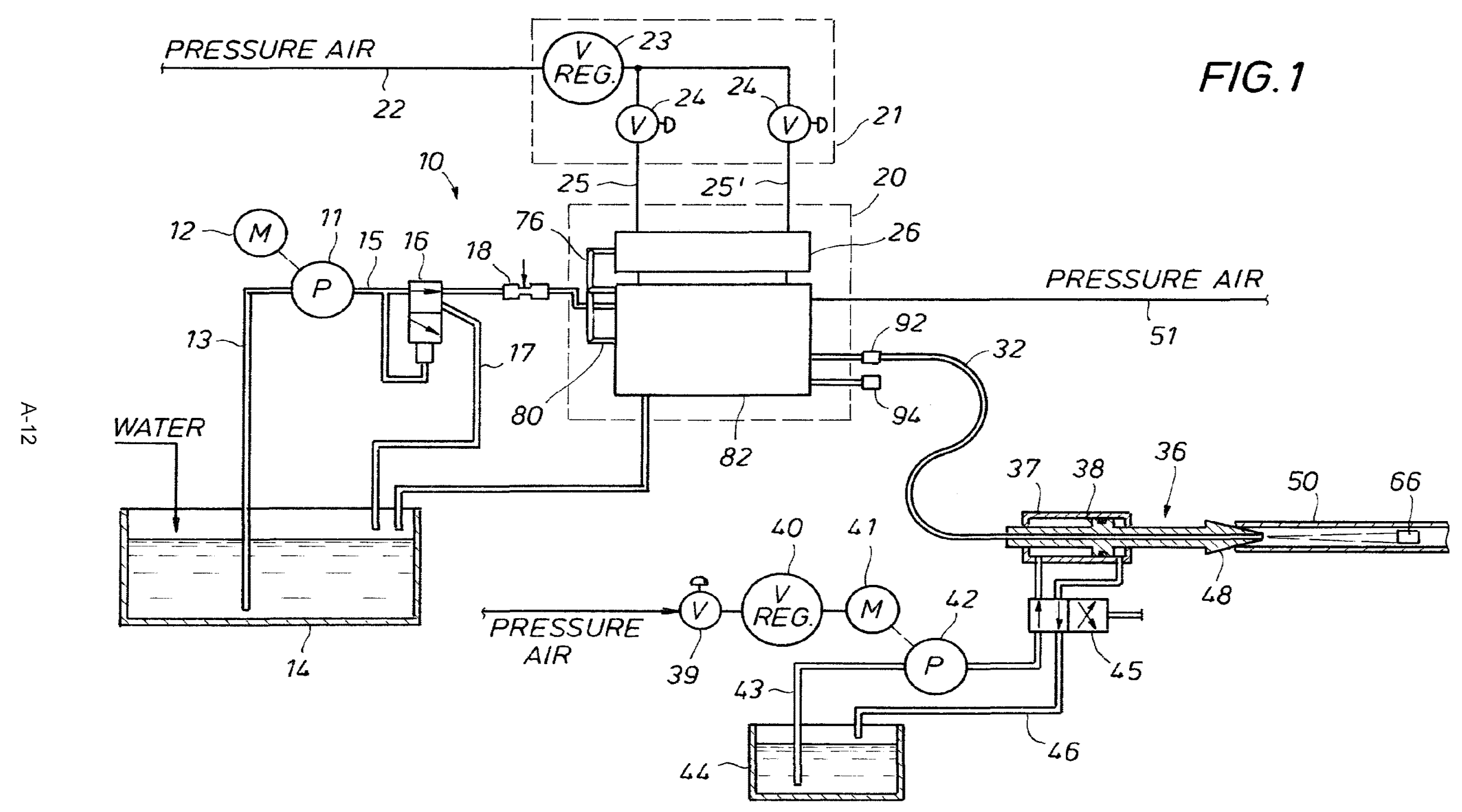

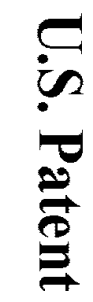


U.S. Patent Sep. 9, $2008 \quad$ Sheet 2 of $3 \quad$ US 7,421,757 B1

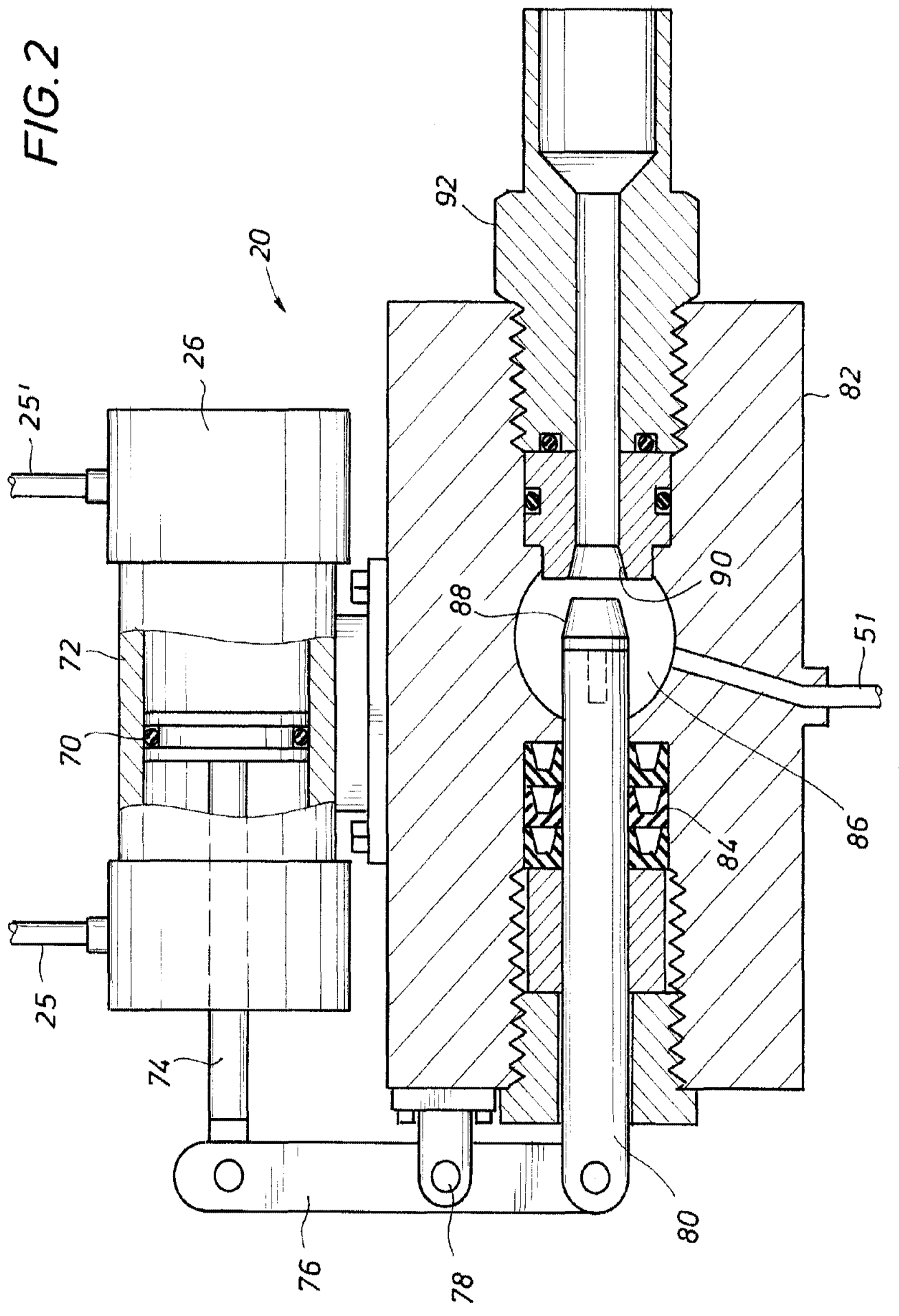



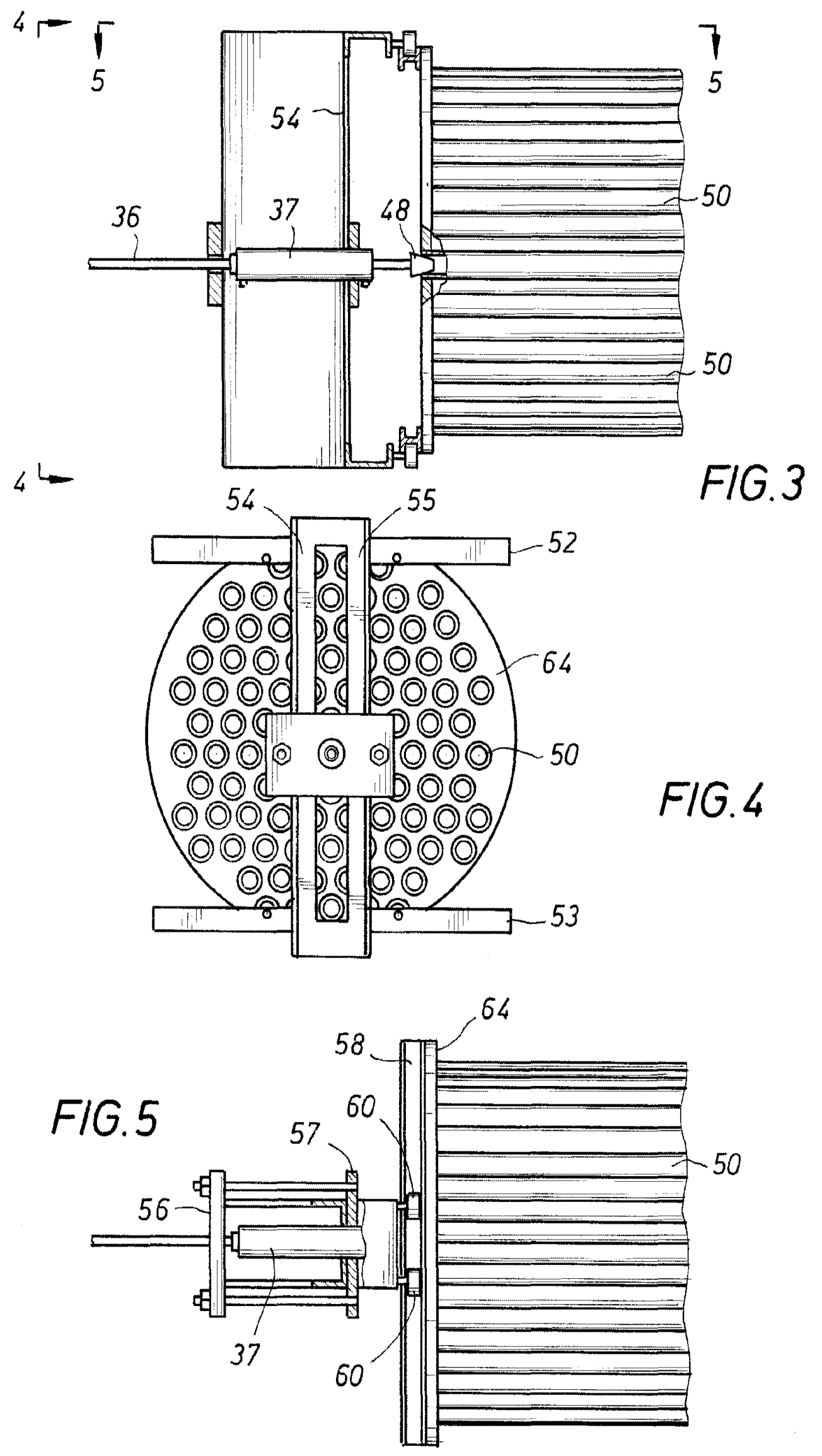


\section{1}

\section{PUMP VALVE MECHANISM}

\section{FIELD OF THE INVENTION}

The present invention relates generally to the field of devices for cleaning clogged heat exchanger tubes and, more particularly to a system for cleaning tubes within a vessel using a pump valve mechanism for mechanically applying variable pressure to material clogging the tubes.

\section{BACKGROUND OF THE INVENTION}

A heat exchanger is normally formed of a plurality of tubes oriented generally parallel to one another. In normal operation, a fluid to be heated or cooled is delivered through the inside of the tubes of such a heat exchanger. The outside surface of the tubes are contacted with a fluid which adds heat or removes heat as required. The plurality of generally parallel tubes forms a bundle. A set of end plates, known as heads, commonly support the bundle of tubes at each end.

Heat exchangers usually operate in a continuous fashion, often for months at a time. However, such continuous operation may be periodically interrupted to clean the tubes. The cleaning process is necessary to remove residue which collects on the inside surface of the tubes which reduces their heat transfer capability. The tubes are normally formed of metal which has a relatively high thermal conductivity. The material which may coat the interior of the tubes, however, has a much lower thermal efficiency for heat transfer. Therefore, the coating formed on the interior of the tubes is detrimental to the efficiency of the operation of the heat exchanger.

As residue builds up on the inside surface of a heat exchanger tube, the tube becomes less and less efficient. One way to counteract this effect is to raise the temperature differential across the tube. However, there are limits to this solution. For instance, the metal used in the tubes of the heat exchanger has a limited capacity for heat as a result of metallurgical considerations. Exceeding the design temperature differential across through the tubes increases fatigue and therefore reduces the useful lifetime of the heat exchanger.

In a well known U-tube design, the bundle of tubes takes a $180^{\circ}$ bend or elbow at more or less the mid-point of the respective tubes. Fluid enters an inlet box which is separated by a divider plate from an outlet box. The fluid then flows through the head, through the tubes in first one direction then the reverse direction, back through the head and finally into the outlet box on the other side of the divider plate. Cleaning the tubes involves removing the accumulated coating material on the inside of the tubes and the difficulty of cleaning the inside surfaces of the tubes is exacerbated by the bend in the tubes. Also, as exchanger designs have improved, the effective length of the tubes has increased. This makes the task of cleaning the tubes more difficult because the long and relatively narrow tubes do not permit easy access to the tubes.

One way that the tubes can be cleaned is by pumping water 55 or perhaps chemically active solvents into the tubes. That is successful but it has limitations. Moreover, since a typical heat exchanger includes a large number of tubes, it is necessary to undertake the cleaning in a repetitive fashion so that a large number of tubes can be cleaned.

In my U.S. Pat. No. 5,423,917, I described a system and a method for cleaning heat exchanger tubes. The system described therein has proven very successful. However, the system includes a control panel with a ganged set of valves to set up a shock wave to be injected into a tube. For particularly stubborn and tenacious fowling, especially involving hundreds of tubes, this manual alignment of the control panel

\section{2}

valves can become tiresome and tedious. Therefore, there remains a need for a system like that described in the ' 917 patent but that mechanizes the shock wave generation process. The present invention is directed to solving this need in the art.

\section{SUMMARY OF THE INVENTION}

The present invention solves this need in the art by provid$10 \mathrm{ing}$ a pump/valve mechanism for directing a shock wave to a tube that is to be cleaned. A pump takes a suction from a sump and the pump discharges to an output and then through a valve which is switched to deliver water under pressure through a controllable orifice. The orifice delivers the water under pres5 sure to the pump/valve mechanism which alternately directs pressurized water to the tube and to an overflow discharge.

In a preferred embodiment, the pump/valve mechanism is actuated pneumatically by regulated air pressure. However, any convenient and appropriate actuation means may be used.

20 In the rest of the tube cleaning system, a lance is provided to deliver pressurized water and the shock wave to the tubes. The lance is positioned by a ram, which cooperates with the lance to align the lance with individual tubes. The lance is directed in an $X$ and $Y$ pattern by a control mechanism to align 5 with selected tubes. The lance is able to travel forwardly in the $Z$ direction. It is constructed on a piston and cylinder mechanism which enables hydraulic control of lance insertion and retraction.

When the lance is inserted, the tip of the lance is placed in the particular tube to be cleaned. Hydraulic control enables rapid indexing of the lance to the left and right to align with the proper tube and to insert into that tube once alignment has been accomplished. The tip of the lance is profiled so that it forms a fairly quick seal with the end of the tube. The lance is 5 hollow to deliver liquid through the end of the lance into the tube. A pressurized air supply provides air to the pump/valve mechanism to purge the tube to be cleaned to set the tube up for a water hammer action when the lance is pressurized with water or cleaning solution.

A pressure surge is set up by timed operation of the pump in cooperation with the orifice. Moreover, this delivers a flow of water into the tube. By appropriate shock wave creation with a mix of air and water injected violently into the tube, the corrosion materials collected on the inside of the tube are fractured and break away. There is a rapid flow of multiphase fluid through the tube. This rapid flow agitates the corrosion residue with sufficient shock tremors that the corrosion residue is broken and will flake off the wall. The loosened material is then flushed out of the tube by the continued flow of fluid from the lance.

These and other features of the present invention will be readily apparent to those skilled in the art from a review of the following description with the accompanying drawings.

\section{BRIEF DESCRIPTION OF THE DRAWINGS}

So that the manner in which the above recited features, advantages and objects of the present invention are attained 60 and can be understood in detail, more particular description of the invention, briefly summarized above, may be had by reference to the embodiments thereof which are illustrated in the appended drawings.

It is to be noted, however, that the appended drawings 65 illustrate only typical embodiments of this invention and are therefore not to be considered limiting of its scope, for the invention may admit to other equally effective embodiments. 
US 7,421,757 B1

3

FIG. 1 is a schematic flow diagram of the system wherein the pump valve mechanism of the present invention finds application;

FIG. 2 is a section view of the pump/valve mechanism of this invention;

FIG. 3 is a side view of a lance mounting mechanism showing a lance which extends to seat against a tube to enable tube cleaning;

FIG. 4 is a sectional view along the line 3-3 of FIG. 3 and shows details of construction of the mechanism which aligns the lance with a particular tube for cleaning; and

FIG. 5 is a sectional view along the line 4-4 of FIG. 3 showing details of construction of the lance insertion mechanism.

\section{DETAILED DESCRIPTION OF PREFERRED EMBODIMENTS}

Attention is now directed to FIG. 1 of the drawings which illustrates a schematic of a system $\mathbf{1 0}$ for cleaning tubes and the like. The system includes a pump $\mathbf{1 1}$ driven by a suitable motor 12 of substantial power. The pump 11 takes a suction through a feed line $\mathbf{1 3}$ from a water sump or reservoir $\mathbf{1 4}$. Water level is maintained in the sump by occasional replenishment. Moreover, the water is typically pure but it can be used with additives. For instance, certain types of acids or bases can be added to accomplish chemical attack on the material to be removed.

The pump 11 has a pump output 15 which is provided to a control valve 16. The control valve 16 is a two position valve. In the illustrated position, water under pressure is delivered from the pump through an adjustable orifice 18. Alternatively, the valve 16 connects with a line 17 which provides a return to the sump. The orifice 18 provides a control signal to manifold $\mathbf{2 0}$ of a pump valve mechanism represented in phantom in FIG. 1 and described in greater detail below.

The manifold operates in conjunction with an air pressure manifold 21. Pressurized air is provided on an air line $\mathbf{2 2}$ into a regulator valve $\mathbf{2 3}$ in the air pressure manifold. The regulator valve 23 provides a regulated air pressure output through a pair of control valves 24 . The control valves 24 are each of the same construction and connect in parallel at the output of the regulator $\mathbf{2 3}$. The manifold $\mathbf{2 1}$ may be replaced with other actuation means, including a hydraulic actuator, an oscillating electric switch, a gas pilot valve, or other means to control a pump/valve mechanism in the manifold $\mathbf{2 0}$.

The control valves $\mathbf{2 4}$ in the manifold are input to the manifold 10 which includes the pump/valve mechanism. Specifically, the control valves $\mathbf{2 4}$ provide air inlet lines $\mathbf{2 5}$ and 25 , respectively, to either side of an actuator 26. FIG. 2 provides greater detail of the pump/valve mechanism. As previously stated, the pump valve mechanism may be operated by any appropriate and convenient actuation means, but the pneumatic actuator is the preferred means and is illustrated.

As shown in FIG. 2, the air inlet lines $\mathbf{2 5}$ and $\mathbf{2 5}$ provide air pressure into the actuator $\mathbf{2 6}$. In the preferred embodiment, the actuator comprises a piston $\mathbf{7 0}$ within a cylinder $\mathbf{7 2}$. Air pressure ported to the air inlet line 25 moves the piston to the right as seen in FIG. 2, and air pressure ported to the air inlet line $25^{\prime}$ moves the piston to the left. The piston 72 is coupled to a piston rod 74 which terminates at a linkage 76 . The linkage $\mathbf{7 6}$ pivots about a fulcrum $\mathbf{7 8}$ and links to a valve rod 80. The valve rod $\mathbf{8 0}$ is mounted for movement within a pump/valve block 82 . Together, the actuator $\mathbf{2 6}$, the block $\mathbf{8 2}$ and the parts associated therewith form the pump/valve mechanism of the invention.

\section{4}

A packing 84 seals around the valve rod 80 where it then enters a manifold 86 . The valve rod 80 terminates in a valve disc $\mathbf{8 8}$ which is configured to seat against a valve seat $\mathbf{9 0}$. When the disc $\mathbf{8 8}$ is off the seat $\mathbf{9 0}$, fluid under pressure from the manifold 86 is free to flow out an outlet fitting $\mathbf{9 2}$.

Referring again to FIG. 1, the pump 11 provides fluid under pressure through the orifice $\mathbf{1 8}$ to the block 82 where it pressurizes the manifold 86 . Depending on the position of the valve rod 80 , fluid flows from the block 82 to the outlet fitting 92 or a waste discharge 94 .

The pump/valve mechanism has appropriate fittings on it to enable connection of a lance feed line 32. The line 32 extends some distance, typically from 10 to 50 feet. Preferably the length of the line is kept relatively short so that pressure 15 surges are not damped in the flow line.

The line 32 feeds fluid, including shock waves, to a lance 36. The lance 36 is coaxial with an elongate cylinder 37 which encloses a piston $\mathbf{3 8}$. The piston $\mathbf{3 8}$ is moved under hydraulic pressure in a double acting construction. This enables positive insertion and retraction of the lance. The hydraulic system preferably uses air from a suitable air pressure source delivered through a control valve 39 which connects to an air pressure regulator 40 . The air pressure is regulated and provided to an air motor $\mathbf{4 1}$. The motor in turn is driven by the air 5 to operate a hydraulic pump $\mathbf{4 2}$.

An inlet line $\mathbf{4 3}$ connects to hydraulic oil sump 44 . Hydraulic oil is delivered to a control valve $\mathbf{4 5}$ to control the movement of the lance.

Specifically, the lance is extended when the valve is in the illustrated position. The lance is retracted when the valve moves to the opposite position. A return line $\mathbf{4 6}$ returns the low pressure oil to the sump. The valve is connected so that power is applied for extension of the lance and for retraction of the lance on operation of the valve. There is also additional 35 equipment for positioning of the cylinder 37 as described below.

As shown in FIG. 1, the lance has an elongate rod portion which terminates at a tip 48 . The lance tip is sized to nest in the end of a tube 50. A seal is made when the tube and tip make contact. The seal enables fluid to be introduced under pressure into the tube $\mathbf{5 0}$.

An air inlet line 51 introduces pressurized air into the block $\mathbf{8 2}$ and into the manifold $\mathbf{8 6}$. This permits the system to blow air through a tube to be cleaned prior the introduction of a 5 shock wave of fluid from the system, thereby providing a water hammer to enhance the clearing effect of particularly stubborn blockages in tubes.

The lance $\mathbf{5 0}$ is moved with respect to a set of tubes in a fashion shown in FIGS. 3, 4, and 5. FIG. 3 shows the lance 36 5 which is supported and aligned by cylinder 37 . It is mounted so that it travels on a pair of parallel rails $\mathbf{5 2}$ and $\mathbf{5 3}$ shown in FIG. 4 of the drawings. These permit movement in the $\mathrm{X}$ direction. The rails are parallel steel beams supported on rollers. A bracket is comprised of left and right frame mem55 bers $\mathbf{5 4}$ and $\mathbf{5 5}$ which move as a unit. They enable vertical movement of the cylinder 37 .

More specifically, the frame members 54 and 55 define a gap where the lance extends through the gap. The cylinder 37 is anchored to the spaced plates $\mathbf{5 6}$ and $\mathbf{5 7}$ which capture the 60 cylinder. The cylinder extends into a pair of guide surfaces and is supported against these guide services for controlled movement. The guide surfaces are formed along the edges of the frame members $\mathbf{5 4}$ and $\mathbf{5 5}$ and thus define the channel $\mathbf{5 8}$ shown in FIG. 5 of the drawings. Rollers at 60 are located in 5 this channel. There are typically four rollers, two at each corner as shown in FIG. 5 , and a corresponding duplicate pair on the opposite side. 


\section{US 7,421,757 B1}

\section{5}

The several rollers guide the cylinder $\mathbf{3 7}$ for movement as illustrated. When it moves up or down, it is guided by the rollers 60 which clamp on the outside of the parallel frame members $\mathbf{5 4}$ and 55. As previously mentioned, the frame members are able to move as a unit to the left or right as viewed in FIG. 4. While this provides one dimension of movement, the movement in the vertical direction in FIG. 4 is the second dimension of movement. When the cylinder $\mathbf{3 7}$ is extended, the lance is moved in the $Z$ direction toward the tubes $\mathbf{5 0}$.

Attention is now directed to FIG. 3 of the drawings where it shows the nozzle $\mathbf{4 8}$ at a particular tube $\mathbf{5 0}$. The tube $\mathbf{5 0}$ is one of many. In fact, hundreds of tubes can be constructed in the heat exchanger. The heat exchanger is defined by a head 64 better shown in FIG. 4 of the drawings. The extendible lance is forced against one of the tubes. The heat exchanger tube $\mathbf{5 0}$ is temporarily plugged by a plug $\mathbf{6 6}$ shown in FIG. 1 to perform the method of this invention.

In the practice of this method, the first step is to temporarily plug the tube 50 with the plug. The plug can leak somewhat. It is not important that it maintain a perfect seal; in fact, it is desirable that it provides some leakage so that the plug restricts flow but does not totally block fluid flow. The plug serves as a liquid flow barrier. Preferably it has a length equal to the diameter of the tube plus a friction of an inch greater length. If it were longer, it would work equally well, but it would also cause more frictional drag while the plug moves along the tube 50. In cleaning the tubes, the plug 66 is first placed in a tube and the lance is moved in an $\mathrm{X}$ and $\mathrm{Y}$ coordinate system until it is aligned with that particular tube. Then, the lance is extended and seats against the tube that has been plugged and the lance seats against the tube with a water-tight seal. As previously described, the tube is then blown free with pressurized air using air from the line $\mathbf{5 1}$.

The next step is to fill the tube with water. This is accomplished by pressurizing the manifold 86 from the pump 11 and holding the disc $\mathbf{8 8}$ off the seat $\mathbf{9 0}$. Fluid then flows through the lance to fill up the selected tube $\mathbf{5 0}$. At this point, the system is set up to deliver a series of repeated shock waves from oscillating action of the pump/valve mechanism. Movement of the actuator piston $\mathbf{7 0}$ back and forth moves the valve rod back and forth at the same rate. In the action, the disk and rod act as a pump, forcing flid under pressure with a pressure surge out through the lance. This has the form of a fluid shock which is administered through the solid column of water When that occurs, there is a tube impact which jars the coating materials on the inside of the tube.

\section{6}

When this shock loading is formed in the tube, the plug 66 may leak or may be forced downstream. No particular problem arises from that because water is always being added through the pump output. The incorporation of the orifice 18 coupled with the standing column of water downstream assures that the system transmits into the dirty tube the cleaning shock wave. The shock wave has the form of a change in pressure propagated through the standing column of water. This forms a shock wave which is experienced in the tube but 10 it is not a pressure wave which is built up behind the plug 66 . In fact, it is not normal to use a plug to hold against high pump pressure. The plug is only a sufficient retardant to prevent complete escape of the water. The plug 66 will chatter and skid, moving finally to the far end of the tube 50. The system 5 utilizes a positive displacement pump 11 which enables the system to provide a relatively constant fluid output. As the pressure buildup is formed and is switched by the pumping action of the pump/valve mechanism, the water in the tube serves to break up the coating of material on the inside of the tube.

As a generalization, a representative pressure at the discharge of the pump 11 may exceed $10,000 \mathrm{psi}$. The pressure at the tip of the lance $\mathbf{4 8}$ is preferably also in that range.

The principles, preferred embodiment, and mode of opera5 tion of the present invention have been described in the foregoing specification. This invention is not to be construed as limited to the particular forms disclosed, since these are regarded as illustrative rather than restrictive. Moreover, variations and changes may be made by those skilled in the art without departing from the spirit of the invention.

I claim:

1. A system for cleaning a tube comprising:

(a) a source of fluid under pressure;

(b) a lance adapted to seal against the tube;

5 (c) a pump/valve mechanism to mechanically provide an oscillating pressure wave from the source to the lance, wherein the pump/valve mechanism comprises:

(i) an actuator; and

(ii) a pump/valve block having a valve in the block, the valve coupled to the actuator with a linkage.

2. The system of claim 1 , wherein the block defines a manifold and the valve controls the flow of fluid from the source of fluid under pressure to the lance.

3. The system of claim 2 , further comprising a source of 5 pressurized air into the manifold.

4. The system of claim 1 , wherein the actuator is operated pneumatically. 


\section{Appendix B}

\section{AIMM TECHNOLOGIES' HYDROKINETIC PROCESS TECHNOLOGY} ASSESSMENT 


\section{TOPICAL REPORT}

\section{UNPLUGGING OF HIGH LEVEL WASTE TRANSFER PIPELINES: AIMM Technologies' Hydrokinetic Process Technology Assessment}

Principal Investigator:

David Roelant, Ph.D.

Florida International University Collaborators:

S. Gokaltun, Ph.D.

Tomas Pribanic, M.S.

Dwayne McDaniel, Ph.D., P.E.

Jose Varona, M.S.

Romani Patel, M.S.

Prepared for:

U.S. Department of Energy Office of Environmental Management Office of Science and Technology Under Grant No. DE-FG01-05EW07033 


\section{DISCLAIMER}

This report was prepared as an account of work sponsored by an agency of the United States government. Neither the United States government nor any agency thereof, nor any of their employees, nor any of its contractors, subcontractors, nor their employees makes any warranty, express or implied, or assumes any legal liability or responsibility for the accuracy, completeness, or usefulness of any information, apparatus, product, or process disclosed, or represents that its use would not infringe upon privately owned rights. Reference herein to any specific commercial product, process, or service by trade name, trademark, manufacturer, or otherwise does not necessarily constitute or imply its endorsement, recommendation, or favoring by the United States government or any other agency thereof. The views and opinions of authors expressed herein do not necessarily state or reflect those of the United States government or any agency thereof. 


\section{TABLE OF CONTENTS}

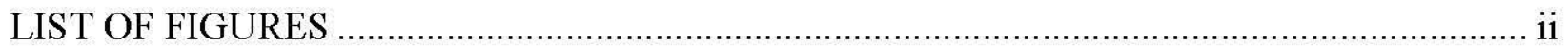

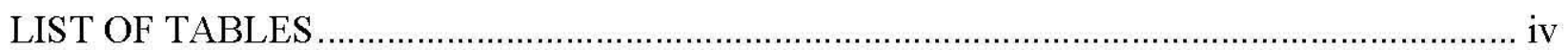

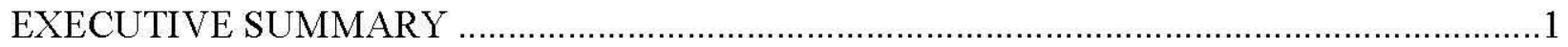

INTRODUCTION

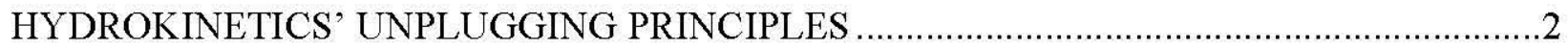

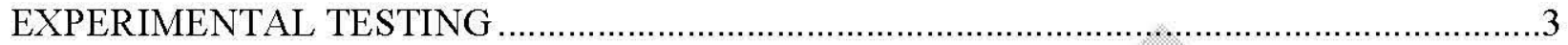

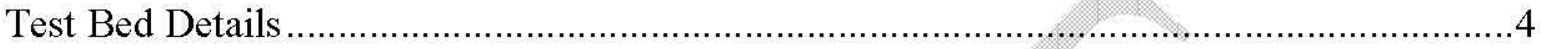

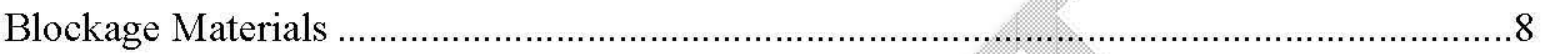

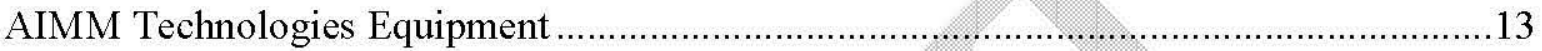

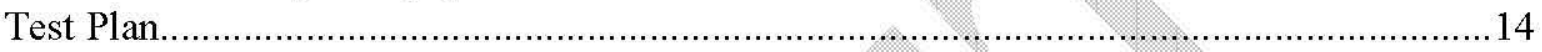

RESULTS …

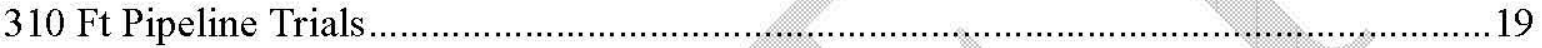

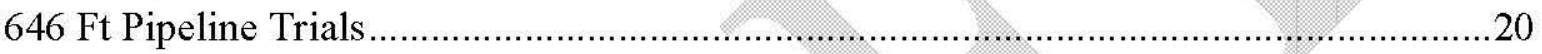

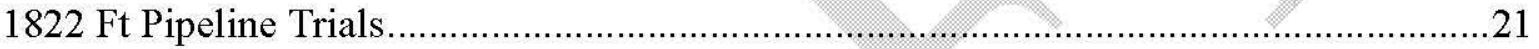

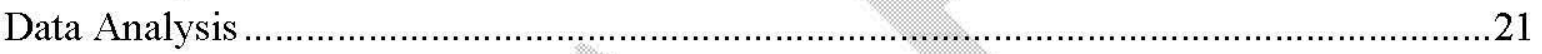

Effect of Pipeline Length and Geometry on Pressure.....................................................23

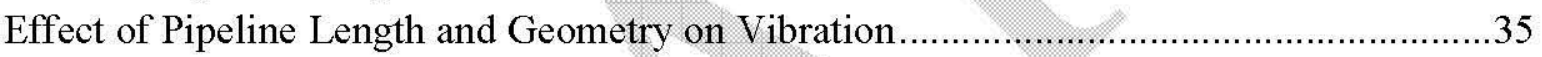

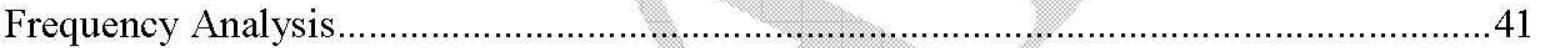

CONCLUSIONS

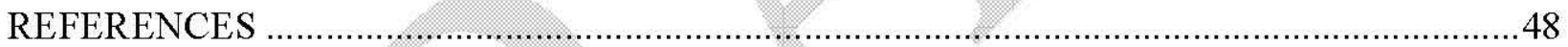


Figure 1. Schematics of $310 \mathrm{ft}$ test bed (Dimensions not to scale).......................................... 4

Figure 2. Schematic of $646 \mathrm{ft}$ test bed (Dimensions not to scale). ………………................. 5

Figure 3. Schematic of the $1822 \mathrm{ft}$ test bed (Dimensions not to scale). ..................................... 5

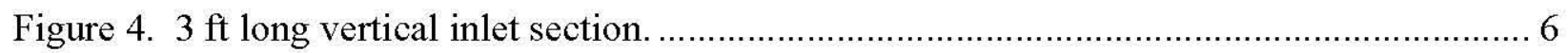

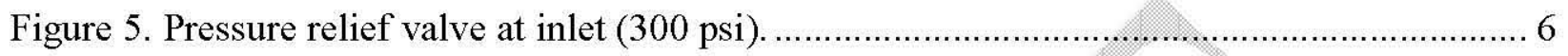

Figure 6. Installation of pressure and temperature sensors on the pipeline.................................... 7

Figure 7. Installation of accelerometer on the pipeline.......................................................... 7

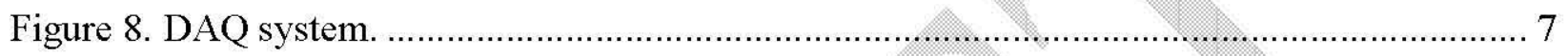

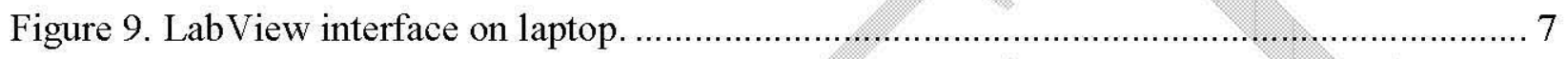

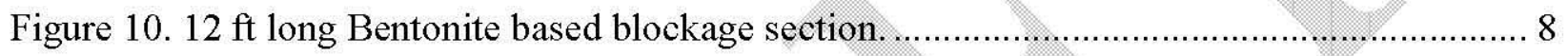

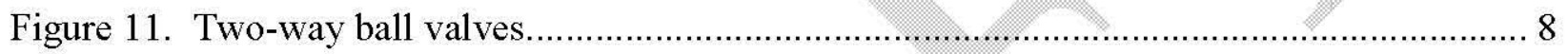

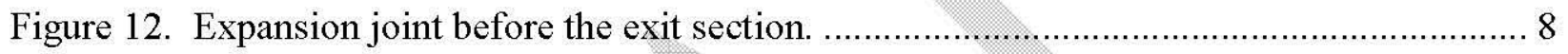

Figure 13. Extrusion test bed with Bentonite plug. ……….................................................. 9

Figure 14. Kmag plug falling off during extrusion test. ........................................................ 9

Figure 15. 4ft Kaolin 66\% after extrusion test.............................................................. 9

Figure 16. Na-Al-Si plug during extrusion test. ………..................................................... 9

Figure 17. Phosphate plug during extrusion test .................................................................... 9

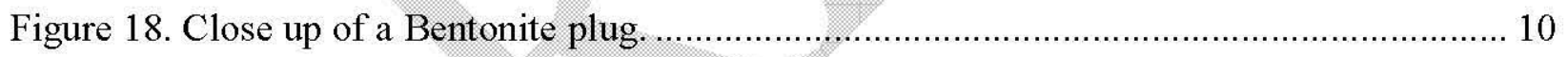

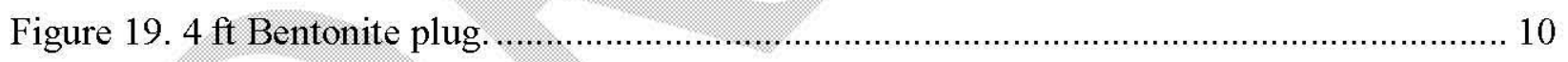

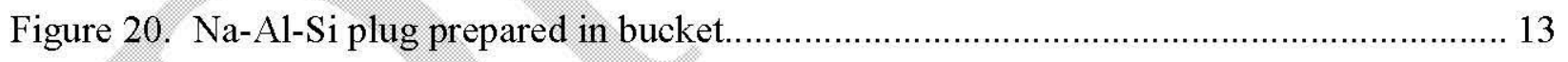

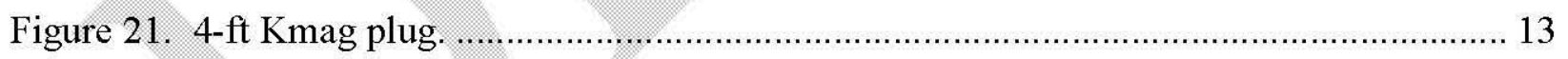

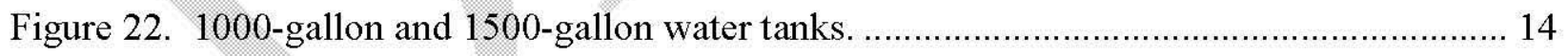

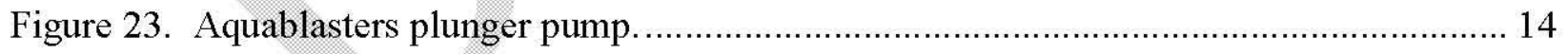

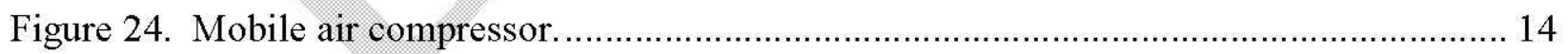

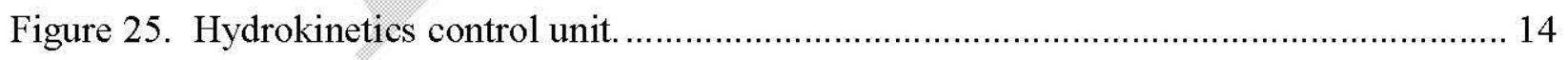

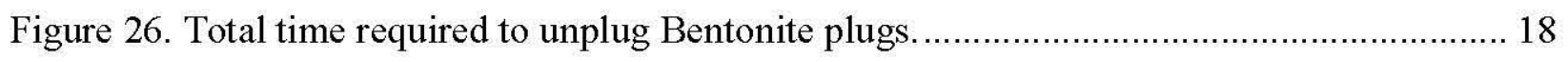

Figure 27. Unplugged $8 \mathrm{ft}$ Bentonite plug at $310 \mathrm{ft}$ pipeline (top). Unplugged blockage section (bottom)

Figure 28. Unplugged $12 \mathrm{ft}$ Bentonite plug (310 ft pipeline). .................................................. 19

Figure 29. 6 in of Kmag unplugged from $2 \mathrm{ft}$ blockage section. ............................................ 20

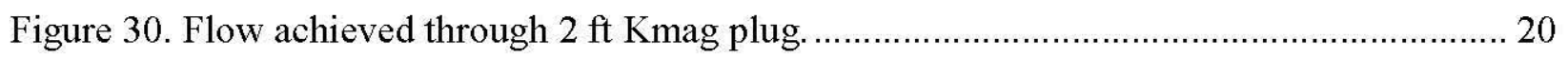


Figure $31.4 \mathrm{ft} \mathrm{Kmag} \mathrm{plug} \mathrm{after} \mathrm{unplugging} \mathrm{trials} \mathrm{with} \mathrm{AIMM.} \mathrm{Front} \mathrm{side} \mathrm{(left)} \mathrm{and} \mathrm{back} \mathrm{side}$ (right). 20

Figure 32. $4 \mathrm{ft} \mathrm{Na-Al-Si} \mathrm{plug} \mathrm{after} \mathrm{unplugging} \mathrm{trials} \mathrm{with} \mathrm{AIMM.} \mathrm{Front} \mathrm{side} \mathrm{(left)} \mathrm{and} \mathrm{back} \mathrm{side}$ (right). 20

Figure 33. Unplugged $12 \mathrm{ft}$ Bentonite plug after trials with AIMM at $1822 \mathrm{ft}$ pipeline. 21

Figure 34. $4 \mathrm{ft} \mathrm{Na-Al-Si} \mathrm{plug} \mathrm{(left)} \mathrm{and} \mathrm{Kmag} \mathrm{plug} \mathrm{(right)} \mathrm{after} \mathrm{unplugging} \mathrm{trials} \mathrm{with} \mathrm{AIMM.} 21$

Figure 35. Typical pressure curve of a successful unplugging trial 22

Figure 36. Pressure data analysis for $8 \mathrm{ft}$ Bentonite plug on the $310 \mathrm{ft}$ test bed. 23

Figure 37. Pressure data for $8 \mathrm{ft}$ Bentonite plug using the $310 \mathrm{ft}$ test bed showing analysis. .... 25

Figure 38. Parametric data for the $310 \mathrm{ft}$ test bed with a target pressure of $150 \mathrm{psi}$.................. 26

Figure 39. Pressure deviation with respect to distance between sensors at three different pipelines during the target zone 30

Figure 40. Pressure deviation with respect to distance between sensors at three different pipelines

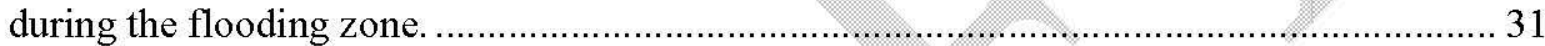

Figure 41. Orientation of accelerometers at the inlet section and plug sections.......................... 36

Figure 42. Parametric vibration data using the $310 \mathrm{ft}$ test bed with a target pressure of $150 \mathrm{psi} .37$

Figure 43. Signal from pressure transducer 1 in time and frequency domain. .......................... 42

Figure 44. Frequencies present during the flooding zone...................................................... 44

Figure 45. Frequencies present on the target zone............................................................... 44

Figure 46. Frequencies present on the $310 \mathrm{ft}$ pipeline ......................................................... 45

Figure 47. Frequencies present on the $646 \mathrm{ft}$ pipeline. ...................................................... 45

Figure 48. Frequencies present on the $1822 \mathrm{ft}$ pipeline........................................................... 46 


\section{LIST OF TABLES}

Table 1. Summary of Extrusion Tests with Various Types of Blockages ................................... 11

Table 2. Chemicals Used to Manufacture $4 \mathrm{ft} \mathrm{Na-Al-Si} \mathrm{Plug} \mathrm{..................................................} 12$

Table 3. Initial Commissioning Test Cases ........................................................................... 15

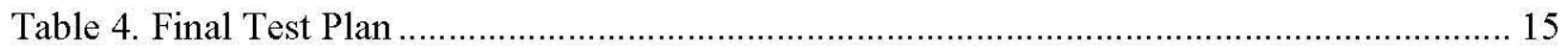

Table 5. Parametric Test Plan at $310 \mathrm{ft}$ with Reducer not Connected .................................. 16

Table 6. Parametric Test Plan at $310 \mathrm{ft}$ with Reducer Connected ............................................ 16

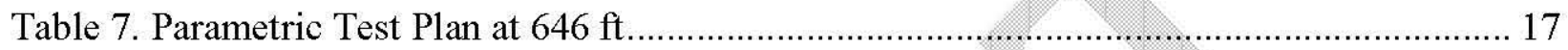

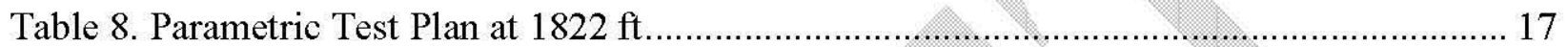

Table 9. Summary of Unplugging Cases in the Final Test Plan .......................................... 18

Table 10. Calculation of Upper and Lower Deviation from Moving Average.......................... 24

Table 11. Effect of Pipeline Length on Moving Average Pressures during Target Pressure Zone

Table 12. Effect of Pipe Length on Maximum Deviation in Pressure from the Mean During

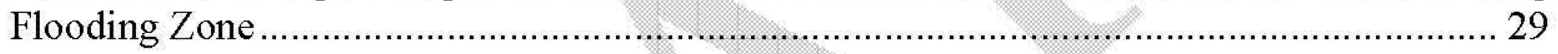

Table 13. Effect of Elbows on Mean Pressures during Target Pressure Zone ........................... 32

Table 14. Effect of Elbows on Pressure Deviations from the Mean during Target Pressure Zone 32

Table 15. Effect of Elbows on Pressure Deviations from the Mean during Flooding................ 32

Table 16. Effect of Expansion Joint (EJ) on Mean Pressures at $1822 \mathrm{ft}$ Test Bed ..................... 33

Table 17. Effect of Expansion Joint (EJ) on Pressure Deviations from the Mean at $1822 \mathrm{ft}$ Test

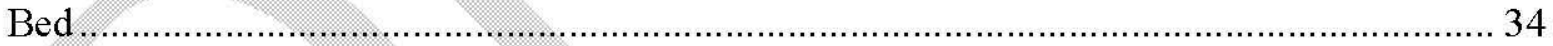

Table 18. Effect of Reducer on Mean Pressure at $310 \mathrm{ft}$ Test Bed at $200 \mathrm{psi}$.......................... 35

Table 19. Effect of Reducer on Pressure Deviations from the Mean at $310 \mathrm{ft}$ Test Bed at $200 \mathrm{psi}$

Table 20. Effect of Pipe Length on Vibration during Target Pressure Zone............................ 37

Table 21. Effect of Pipe Length on Vibration during Flooding Zone ...................................... 38

Table 22. Effect of Expansion Joint (EJ) on Vibration - $1822 \mathrm{ft}$ Test bed, Axis 1 (Outlet/Inlet). 39

Table 23. Effect of Expansion Joint on Vibration - $1822 \mathrm{ft}$ Test bed, Axis 2 (Outlet/Inlet) ....... 40

Table 24. Effect of Expansion Joint on Total Vibration - $1822 \mathrm{ft}$ Test bed ............................... 40

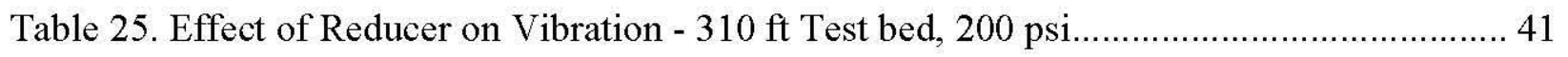

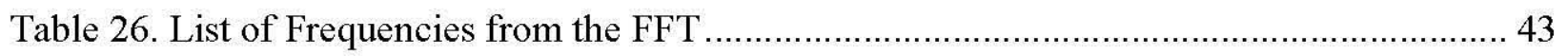




\section{EXECUTIVE SUMMARY}

Pipeline plugging has occurred in the past at DOE sites during high level waste (HLW) transfers, resulting in schedule delays and increased costs. In order to ensure smooth operation of the waste transfers and to ensure tank farm cleanup milestones are met, a pipeline unplugging tool/technology is crucial. Through an industry call various unplugging technologies were tested and evaluated at Florida International University (FIU). Two technologies were identified to have the capability to withstand the rigors of operation in a radioactive environment and with the ability to handle sharp $90^{\circ}$ elbows; NuVision Engineering's Fluidic Wave-action Technology and AIMM Technologies' Hydrokinetics Method. A second phase of testing has been conducted for both technologies with the objective to qualify at least one technology for subsequent deployment at a DOE facility. In this report, the analysis of data collected during the experimental investigation of AIMM Technologies' Hydrokinetics method and conclusions drawn will be presented.

The current phase of testing was comprised of a 3-inch diameter full-scale pipeline instrumented with sensors facilitating data acquisition for performance evaluation and understanding of technology operation, as it applies to three types of plugs typical of DOE HLW. A clay-based plug was created with a Bentonite water mixture, which is typically used in emulating slurry mixes. The other two plugs were crystallized salt plug stimulants: sodium-aluminum-silicate (Na-Al-Si) and potassium-magnesium-sulfate (Kmag). The recipe for manufacturing the Na-AlSi plug was provided by engineers at PNNL. Three different test bed lengths $(310,646$, and 1822 $\mathrm{ft}$ ) were utilized to determine the effectiveness of the Hydrokinetics method with respect to pipe length. Unplugging times were determined for each plug type and at each test bed length in cases where unplugging was achieved.

During the testing trials, AIMM Technologies was more successful removing the Bentonite plugs than the salt-based plugs. In general, the pressure pulses attenuated significantly from the inlet to the blockage section during the unplugging process. The vibrations were also attenuated significantly from the inlet to the blockage during the unplugging process. This suggests that the remaining air within the pipeline and the low operating pressure (below $300 \mathrm{psi}$ for HLW lines) inhibited the ability for AIMM's Hydrokinetics process to operate at its optimum capability.

The significant attenuation of the pressure pulses and vibration suggests that the mechanism for removing the blockages on the successful trials was a combination of the static pressure and the small residual pressure pulses propagated to the blockage. Imposing a vacuum on the pipeline prior to filling would significantly decrease the attenuation rate and improve performance.

Detailed information resulting from the testing will provide the DOE end-user with sufficient data and understanding of the technology, and its limitations so that management decisions can be made whether the technology has a reasonable chance to successfully unplug a pipeline, such as a cross-site transfer line or process transfer pipeline at the Waste Treatment and Immobilization Plant (WTP). 


\section{INTRODUCTION}

As Hanford moves into a more active retrieval and disposal program, the site engineers will be encountering increasing cross-site pipeline transfers with a corresponding increase in the probability of a pipeline getting plugged. In the past, some of the pipelines have plugged during waste transfers, resulting in schedule delays and increased costs. Furthermore, pipeline plugging has been cited as one of the major issues that can result in unplanned outages at the Hanford WTP, causing inconsistent operation. As such, the availability of a pipeline unplugging tool/technology is crucial to ensure smooth operation of the waste transfers and to ensure Hanford tank farm cleanup milestones are met. Previous studies at Florida International University (FIU) included the testing and evaluation of unplugging technologies through an industry call. Two technologies were identified based upon the testing that could withstand the rigors of operation in a radioactive environment and with the ability to handle sharp $90^{\circ}$ elbows. The proposed testing and evaluation of these two technologies extends the technology validation performed earlier and will attempt to qualify one of the pipeline unplugging technologies for deployment at the Hanford site as per the site criteria.

\section{HYDROKINETICS' UNPLUGGING PRINCIPLES}

Based on the pipeline unplugging technology demonstrations conducted at FIU in FY99 to FY02, AIMM Technologies' Hydrokinetics method was one of the two most promising technologies selected for qualification testing for the Hanford site. The operating principles of these technologies are outlined in the following.

The Hydrokinetics method is a patented technology that has been used to clean pipes clogged with fouling materials. It was reported that the mechanical bonds between the plug and the pipe wall are broken by first filling the pipeline with liquid up to the plug and then creating cavitations in the fluid filled pipe by applying pressure pulsations at the inlet (Garcia 1997). The cavitations collapse due to the applied pressure cycles that vibrates the blockage and the pipe wall. The difference in vibration frequencies of the blockage and the pipe wall breaks the mechanical bond between them and the blockage is pushed out by the fluid pressure.

The Hydrokinetics system consists of a water/solvent tank, a plunger type pump, a portable air compressor and a control unit. First, the connections between the air compressor, water pump and pipeline with the control unit are established using high pressure hoses. The water pump is turned on and switched to the desired RPM value. The pressure level in the control unit is adjusted using the pressure regulating valve at the control unit. There are two switches on the control unit that allow the water collected in the control unit to be directed into the pipeline. Using the switches on the control unit, the pipeline is filled at the flow rate value of the pump until the pressure in the pipeline has reached the pressure set at the control unit.

The Hydrokinetics process does not require that a vacuum be created in the pipeline prior to the pipeline being filled. Since the air is not evacuated from the pipe, a two-component fluid system is formed inside the pipeline. Using the switches on the control unit, pressure fluctuations are 
created in the pipeline in a randomized manner. This process is repeated until the blockage is removed.

The frequency and duration of pulsations are set by the AIMM operator, while the pressure of pulsations is set to the maximum pressure permitted.

Benefits of AIMM's Hydrokinetics technology include:

- $\quad$ Short mobilization and demobilization time.

- Can be used to deliver chemical solvent to the blockage where a solvent may be of assistance in loosening a blockage.

- $\quad$ System does not cause pressure amplifications.

- Technology can negotiate many elbows.

- Technology can be operated remotely.

- Unplugging times are short compared to other technologies.

Limitations of AIMM's Hydrokinetics technology include:

- $\quad$ Time to fill the pipeline is long.

- Pulsations are manually controlled and are not automated.

\section{EXPERIMENTAL TESTING}

Process variables important to this study include unplugging rates, pipeline pressure distributions (maximum pipe pressure), and the variability of the distributions with respect to the equipment control parameters. The equipment control parameters, which are provided later in this report, are the parameters AIMM must select to operate their equipment. The effect of these parameters on the pipeline pressure and erosion rates needs to be well understood. In addition, to qualify AIMM's technology, maximum pressures will need to be determined and compared with site safety requirements. Due to the lack of information on exactly how the AIMM's technology scales to longer pipes, we have adopted a parametric approach to evaluate the technology functionality and how it is expected to affect the process variables.

The following data was collected to provide understanding of the technology, its capability, limitations and safety:

- $\quad$ Pressure profile along the test bed, time dependent pressure measurements at several pressure taps along the test bed.

- Temperature of the water in the test bed pipeline.

- $\quad$ Operation time.

- $\quad$ Plug weight - before and after technology operation.

- Unplugging efficiency.

- $\quad$ Control unit pressure and pump RPM. 
Other data from the test bed that was used in the analysis include:

- Distance to the plug, distance from the test bed entry point to the plug.

- $\quad$ Plug length.

- $\quad$ Nature of the plug, composition of the material used to create the plug.

- $\quad$ Number of elbows in the test bed from entry point to the plug.

- Distances between pressure transducers.

\section{TEST BED DETAILS}

Experiments were conducted using three test bed lengths at $310 \mathrm{ft}, 646 \mathrm{ft}$ and $1822 \mathrm{ft}$. Using a non-linear regression analysis, the measurements taken at these three lengths can be used to forecast the performance of the technology to pipeline lengths up to $19,000 \mathrm{ft}$.

The instrumented test bed for technology qualification was designed and constructed with the capability to evaluate the impact of a number of parameters on the technology effectiveness, including: the distance to the plug, pipe layout (e.g., bends, expansions, reducers, etc.).

Schematic diagrams of the test beds are shown in Figure 1 to Figure 3 . The three pipelines are connected to each other using two-way ball valves. This allows switching from one pipeline to another with minimum down time. In Figure 1 to Figure 3, the lines in grey demonstrate the pipes that are not used for that length and the black lines show the pipes that are in use. The test beds were constructed from 3-inch diameter, 21-foot long, Schedule 10, carbon steel pipe sections joined by Victaulic couplings. The pipes were clamped to $4 \times 4$ 's that were fixed to the ground with rebars.

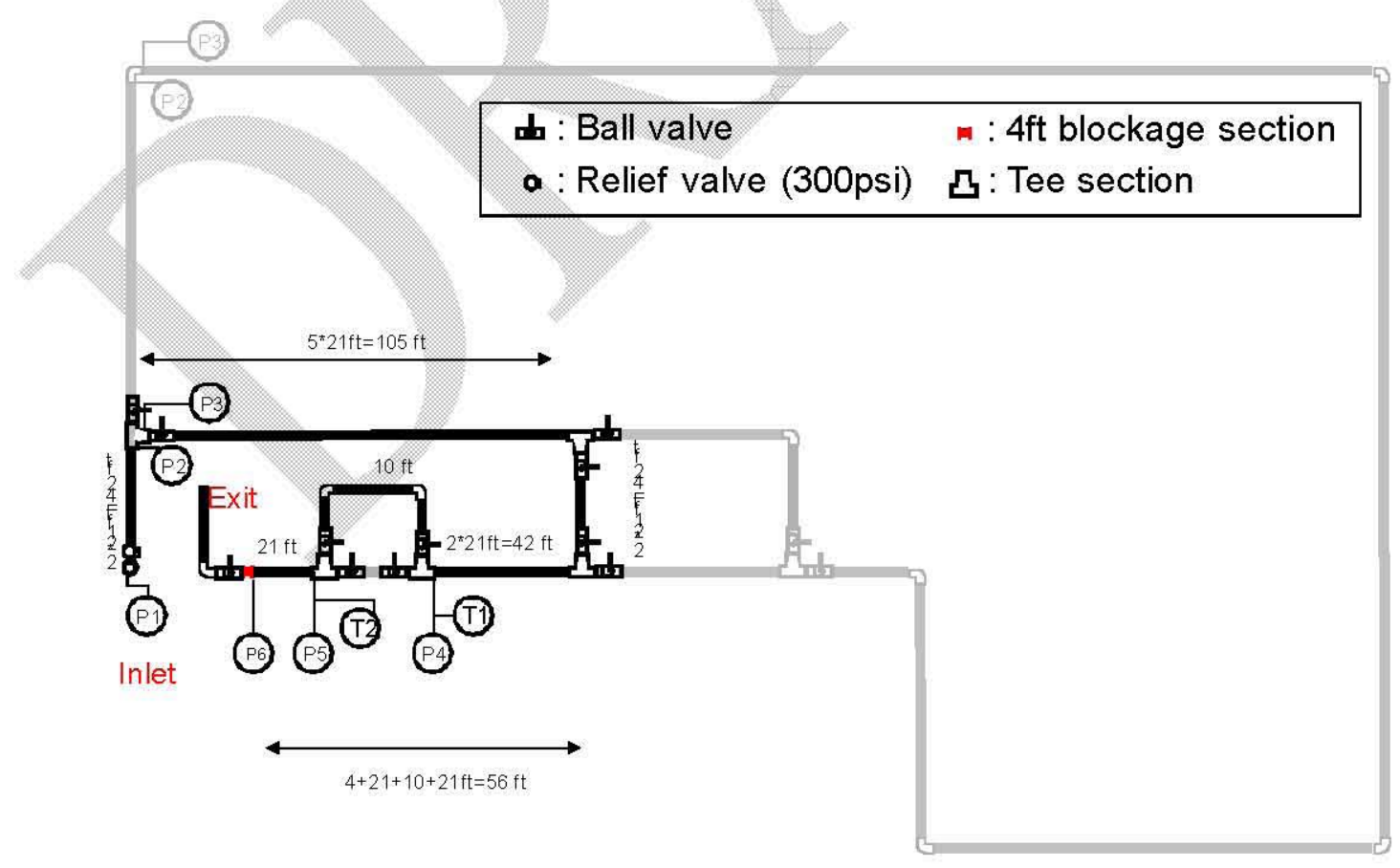

Figure 1. Schematics of $310 \mathrm{ft}$ test bed (Dimensions not to scale). 


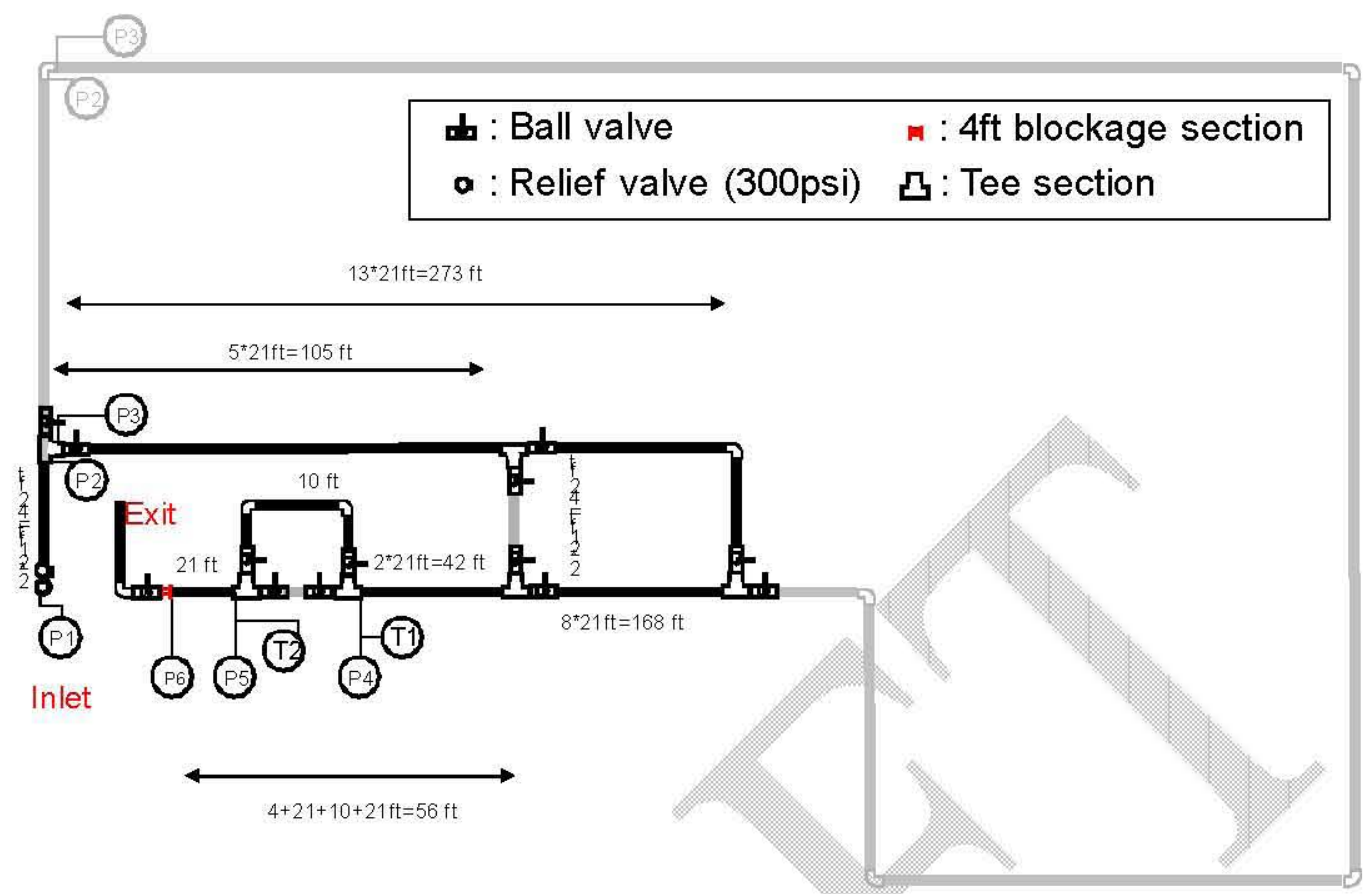

Figure 2. Schematic of $646 \mathrm{ft}$ test bed (Dimensions not to scale).

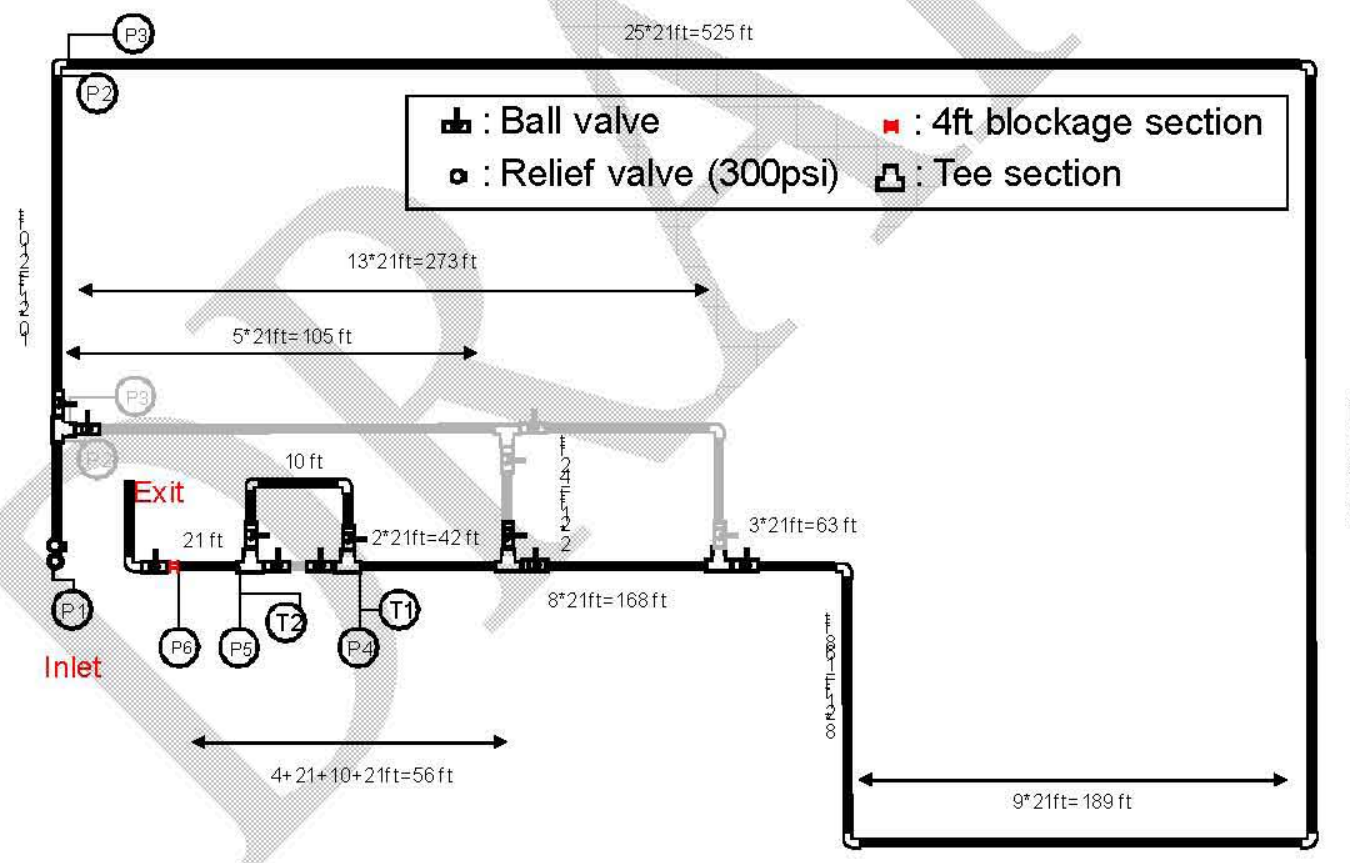

Figure 3. Schematic of the $1822 \mathrm{ft}$ test bed (Dimensions not to scale).

The test bed is designed such that the inlet and exit sections remain in the same location for each of the three test beds. To simulate the connection to the transfer lines in a pit, a $3 \mathrm{ft}$ long inlet section is connected vertically to the long horizontal section via a sharp $90^{\circ}$ elbow (Figure 4). A 300 psi pressure relief valve was placed at the bottom of the inlet section (Figure 5). The relief valve was sized according to the maximum pressure limit in the DOE waste transfer lines. The 
test bed entry point is equipped with a 1 " tee section. In Figure 4 it is shown that a 3-way ball valve was used at the inlet. This ball valve enabled for the connection of the water tank to the pipeline during flooding of the pipes and to AIMM's pump during the unplugging operation.

In Figure 1 to Figure 3, it is shown that pressure transducers were located throughout the test bed. P2 and P3 were placed around a $90^{\circ}$ turn to evaluate the effect of turns on pressure propagation. Similarly P4 and P5 were placed to determine the effect of an expansion joint on the unplugging process. One dynamic and one static pressure transducer were used at each pressure point on the pipeline. The static pressure sensors were Omega PX319-1KGI type with $0-1000$ psig range and had less than $1 \mathrm{~ms}$ of response time. Dynamic pressure sensors were of Omega DPX101-1K type with $0-1000$ psig range and 0.0083 response time. The dynamic sensors provided any fluctuation in nominal pressure that static pressure sensors could not detect. In Figure 1 to Figure 3, T1 and T2 represent the locations where water temperature was recorded. Two K-type thermocouples with $1 \mathrm{sec}$ of response time were used for temperature measurements (Figure 6). Two 34201A type accelerometers with $\pm 2 \mathrm{~g}$ range were used during the tests in order to capture acceleration in three degrees of freedom of the pipes at the inlet and blockage sections (Figure 7). The sensors were connected to a data acquisition (DAQ) system from National Instruments with FPGA capability that allowed recording high speed data (Figure 8) and LabView software was used to observe real-time variation of collected data (Figure 9).

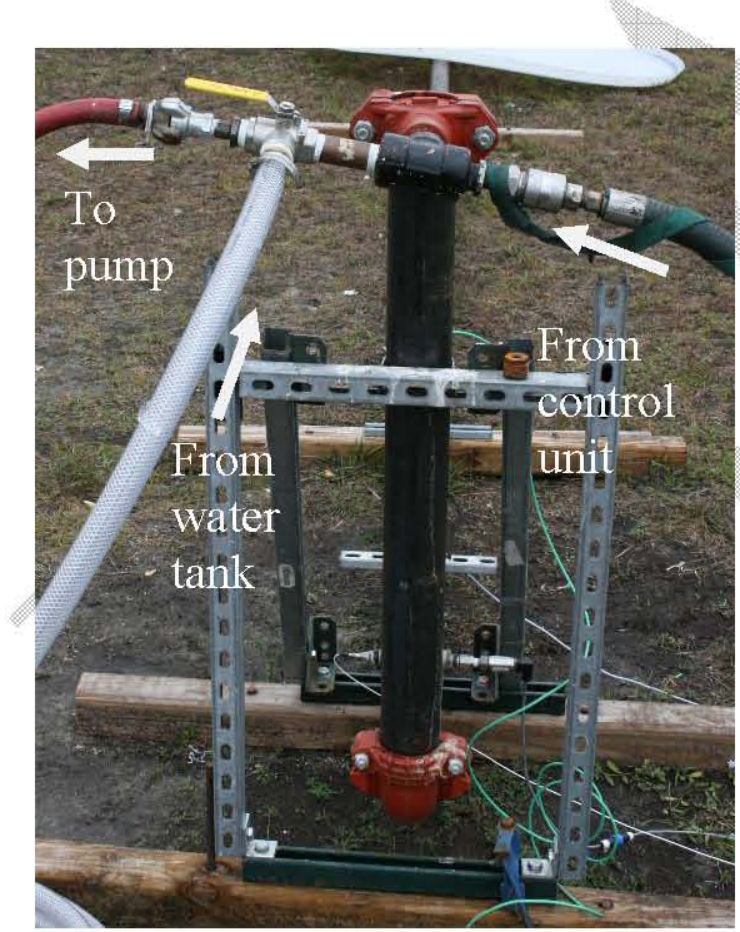

Figure 4. $3 \mathrm{ft}$ long vertical inlet section.

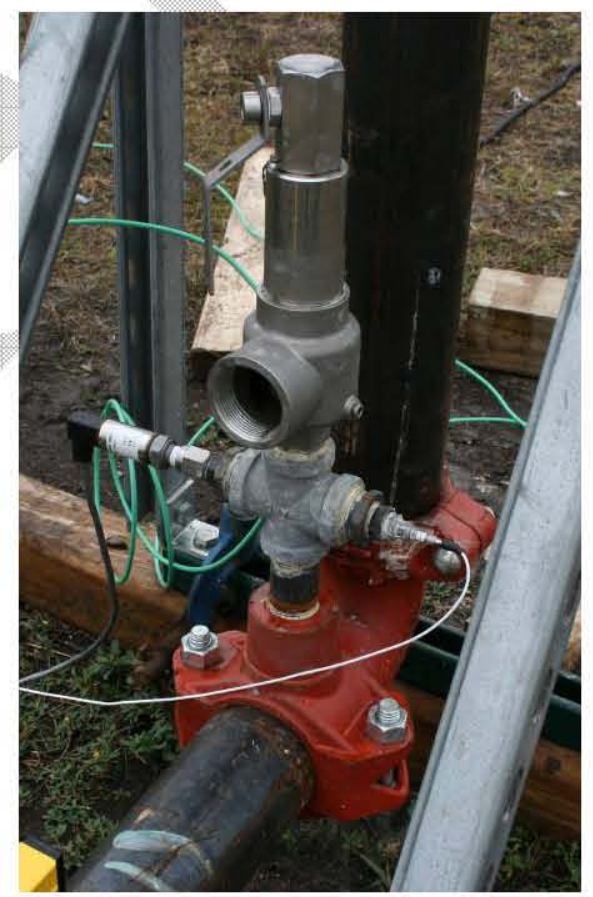

Figure 5. Pressure relief valve at inlet (300 psi). 


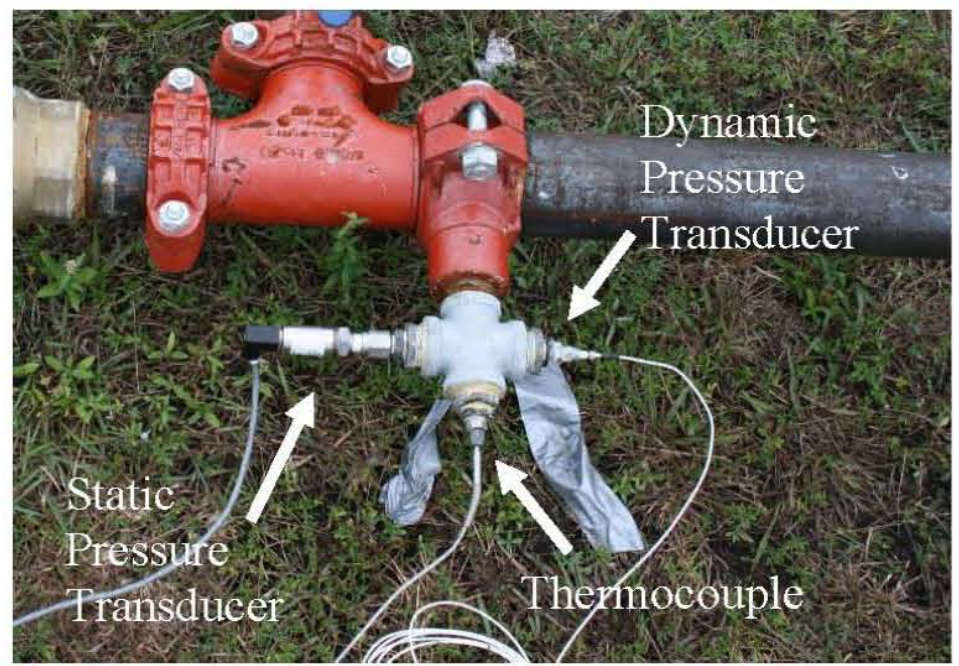

Figure 6. Installation of pressure and temperature sensors on the pipeline.

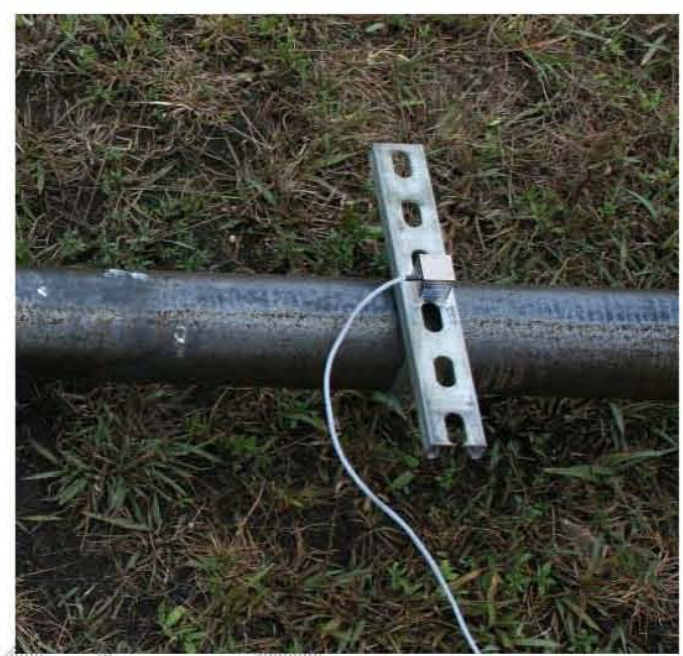

Figure 7. Installation of accelerometer on the pipeline.

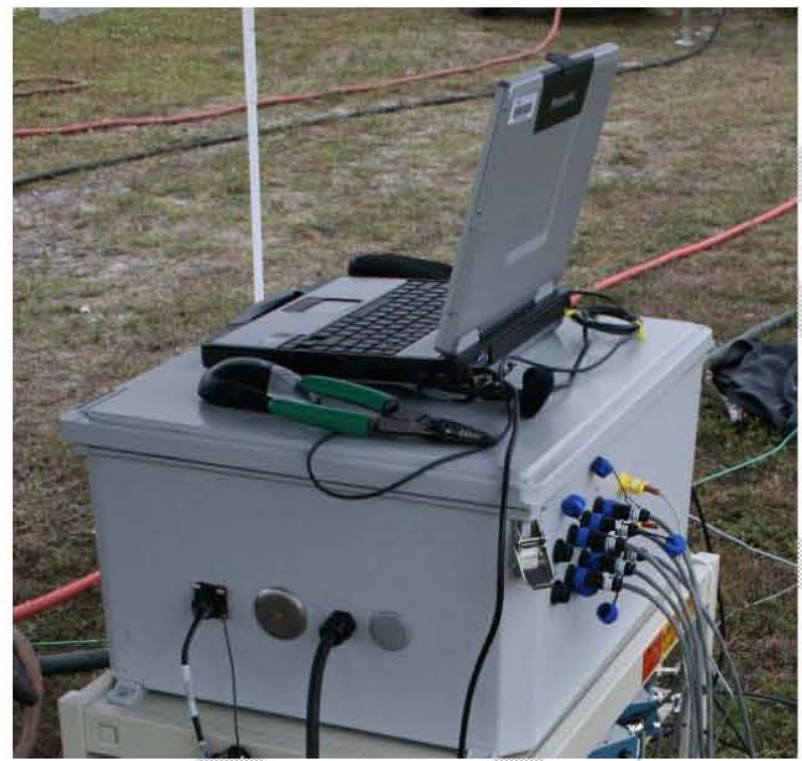

Figure 8. DAQ system.

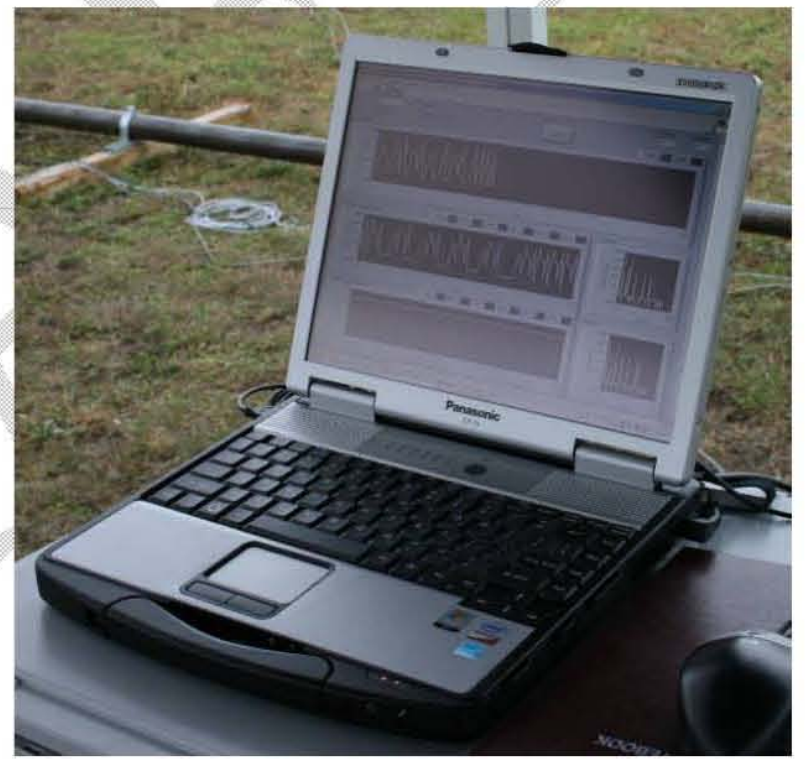

Figure 9. LabView interface on laptop.

For the unplugging experiments, the blockage section was also made out of 3-inch diameter, 4foot long, Schedule 10, grooved-end carbon steel pipes. The blockage sections were connected to the rest of the pipeline using same Victaulic 77 type elastic couplings. In cases where $8 \mathrm{ft}$ or $12 \mathrm{ft}$ blockages were required, multiple $4 \mathrm{ft}$ blockage sections were coupled together to make a longer blockage section (Figure 10). 


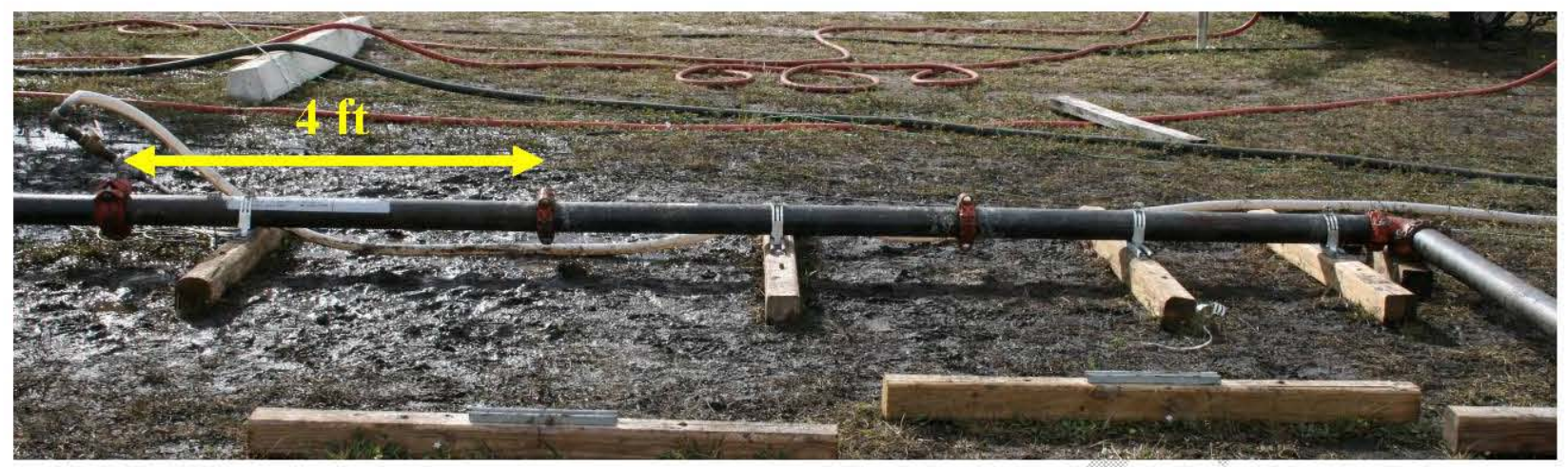

Figure 10. $12 \mathrm{ft}$ long Bentonite based blockage section.

Also shown in the test bed drawings (Figure 1 - Figure 3), was a removable expansion joint located just upstream of blockage section. This joint, containing three 10 - $\mathrm{ft}$ sections, emulates the expansion joints typical of the cross-site lines at Hanford (Figure 12). The expansion joint can be removed in order to evaluate the effectiveness of the technology with and without the joint by using the two-way ball valves shown in Figure 11.

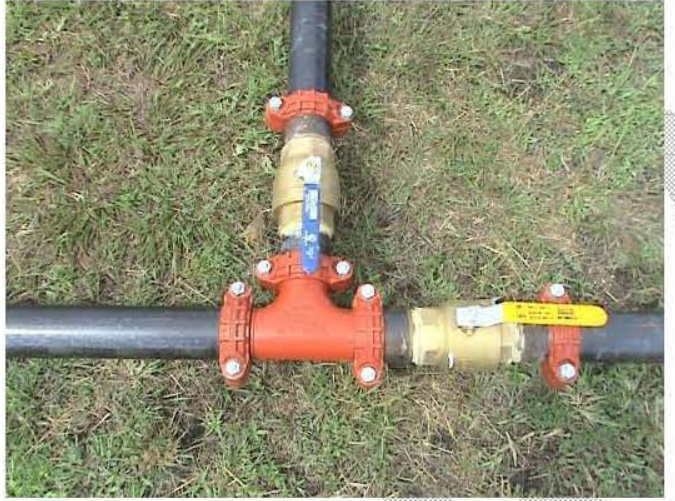

Figure 11. Two-way ball valves.

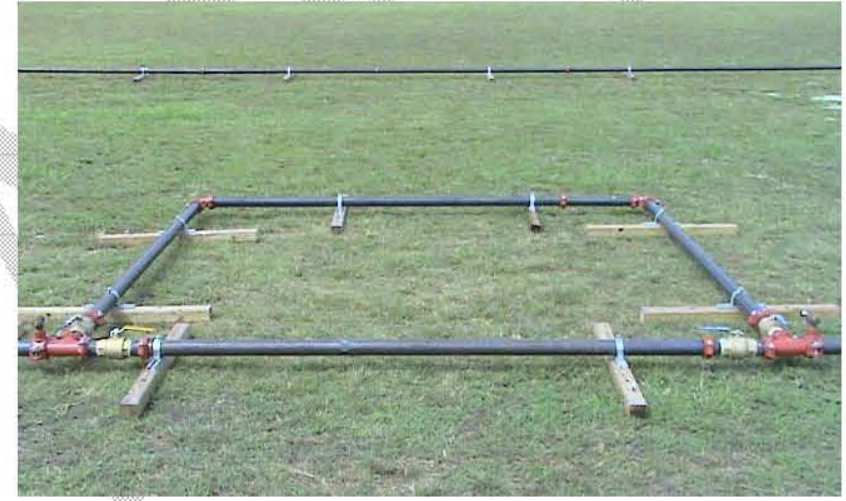

Figure 12. Expansion joint before the exit section.

For one test at $310 \mathrm{ft}$ a constricted section in the pipeline is created by placing a pair of 3 to 2inch reducers to evaluate its effects on the technologies performance The reducer was installed on the $310-\mathrm{ft}$ test bed during parametric testing with no blockage, upstream of the expansion joint.

The various lengths of the test beds were used to evaluate how the effectiveness of the unplugging technology was impacted by the distance to the blockages as well as pipe layout (e.g., bends, expansions, reducers, and elevation changes before and after the plug).

\section{BLOCKAGE MATERIALS}

For the AIMM Technologies unplugging evaluation, three different blockage materials were utilized to simulate various plugging scenarios at DOE sites. The criterion in choosing blockage materials for AIMM Technologies was related to their mechanical strength characteristics. Prior to the testing, AIMM Technologies engineers informed FIU that the minimum pressure level they could operate their systems was at $100 \mathrm{psi}$. In order to evaluate the effectiveness of the 
Hydrokinetics technology, the blockage materials had to be selected such that they would sustain at least pressures of $150 \mathrm{psi}$. In order to prepare for this criterion, various plugs were manufactured and tested on a small extrusion test bed (Figure 13). The extrusion test bed consisted of a hand pump that is rated to $700 \mathrm{psi}$, two pressure gauges, a pressure relief valve and an air release valve.

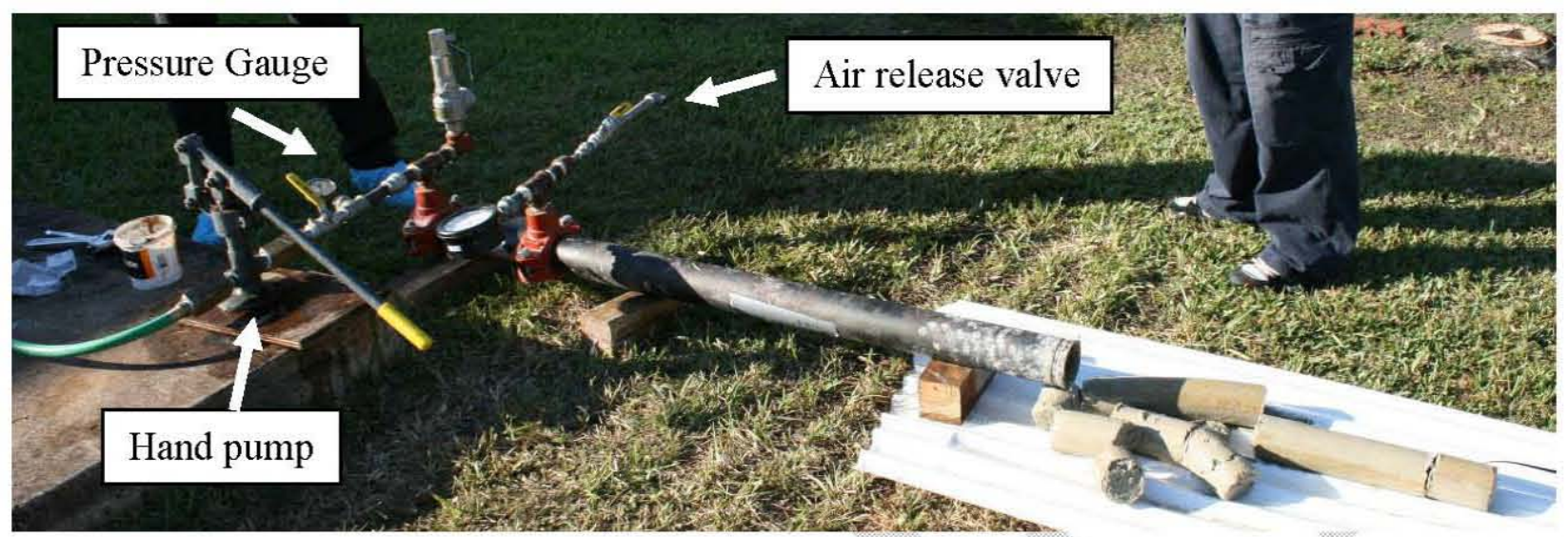

Figure 13. Extrusion test bed with Bentonite plug.

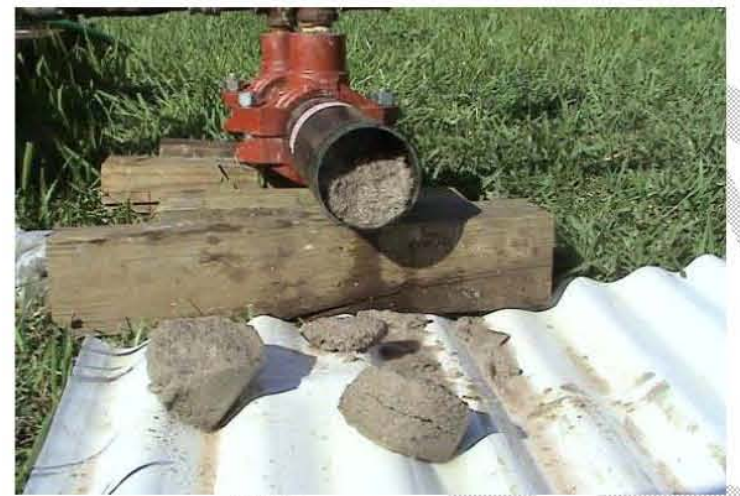

Figure 14. Kmag plug falling off during extrusion test.

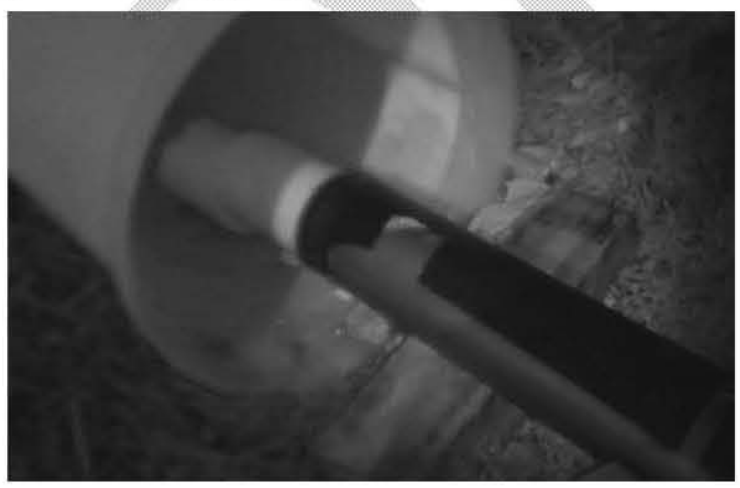

Figure 16. Na-Al-Si plug during extrusion test.

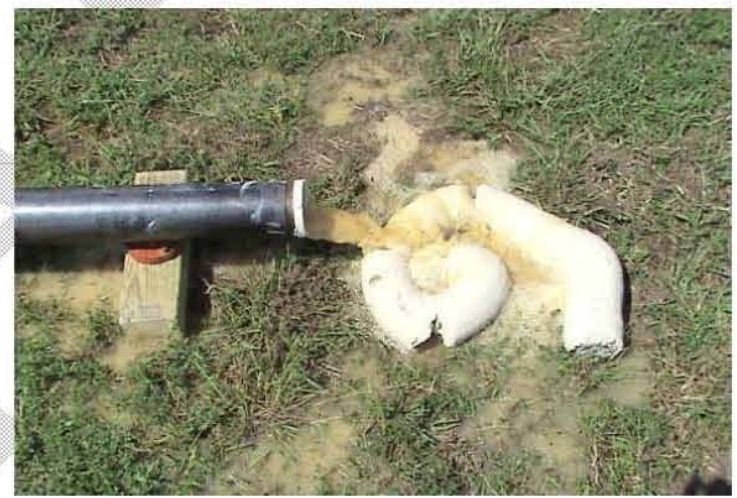

Figure 15. 4ft Kaolin $66 \%$ after extrusion test.

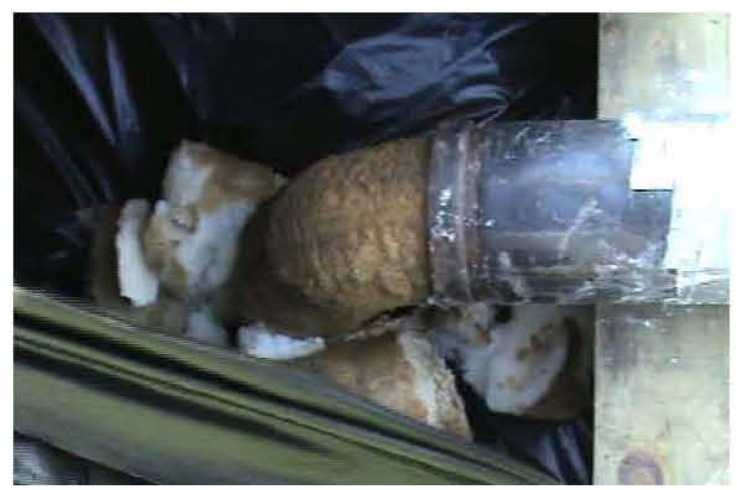

Figure 17. Phosphate plug during extrusion test.

The procedure of the extrusion test is straightforward. The air release valve is opened and the water is turned on. Once the air is removed from the pipe, the release valve is closed and pressure builds up in the system. The pressure is further increased by using the hand pump until 
the plug starts moving. The maximum pressure attained is noted. In case the plug does not come out, the pressure is applied for $15-20$ minutes and then the procedure is halted. The summary of blockage materials tested for extrusion pressures are listed in Table 1.

In Powell et al. (1997) it was reported that shear strength, cohesiveness and water-absorption rate were among key sludge properties that determined the performance of unplugging methods. In previous unplugging tests, Kaolin clay water mixture was used because it was recommended as a sludge simulant by Golcar et al. (1997) and Powell (1996) since it's shear strength, cohesiveness, particle size distribution, and density (at 66-67 wt\% kaolin in water) were similar to those of tank sludge. The shear strength of $66 \%$ Kaolin water mixture is $3.5 \mathrm{kPa}$, which can be multiplied with the surface area to estimate the dynamic friction force applied by the pipe walls on the plug.

When a $4 \mathrm{ft}$ plug of $66 \%$ Kaolin is used, the normal pressure required to unplug it must be at least $12.8 \mathrm{psi}$, which is significantly low for the AIMMs testing. Bentonite clay, however, was a better choice for testing with AIMM Technologies since it had a higher shear strength value $(\sim 19.2 \mathrm{kPa}$ at $66 \%$ ) at same compositions (Powell et al. 1997). For a $4 \mathrm{ft} 68 \%$ Bentonite plug, the static water pressure required to unplug it is found to be at least $190 \mathrm{psi}$ (Table 1).

The bentonite-water mixture was prepared in a large bucket and mixed using a drill attachment until uniformity was achieved (Figure 18 and Figure 19). Four-foot steel pipes that are closed on one side were then completely filled with the mixture by dropping small pieces in and compressing with a long plunger rod. In order to remove air gaps that can get entrapped inside the blockage during filling, the blockages were compressed using a torque wrench.

Plug extrusion tests were also conducted for $8 \mathrm{ft}$ Bentonite plugs. The extrusion pressure for the $4 \mathrm{ft}$ plug, although better than the Kaolin plug, was still low for the testing purposes. The $8 \mathrm{ft}$ Bentonite plugs had extrusion pressures of approximately 300 psi.

The Na-Al-Si and Kmag plugs had significantly higher extrusion pressures. The $4 \mathrm{ft} \mathrm{Na-Al-Si}$ plug had an extrusion pressure of $500 \mathrm{psi}$ and the $2 \mathrm{ft} \mathrm{Kmag} \mathrm{plug} \mathrm{had} \mathrm{water} \mathrm{leakage} \mathrm{at} 450 \mathrm{psi}$. It should be noted that Kmag is water soluble and its extrusion pressure may be time dependent.

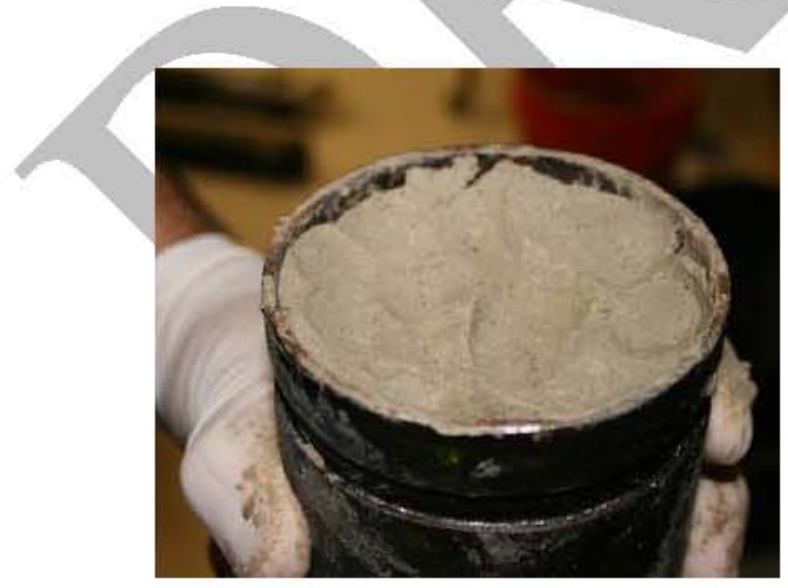

Figure 18. Close up of a Bentonite plug.

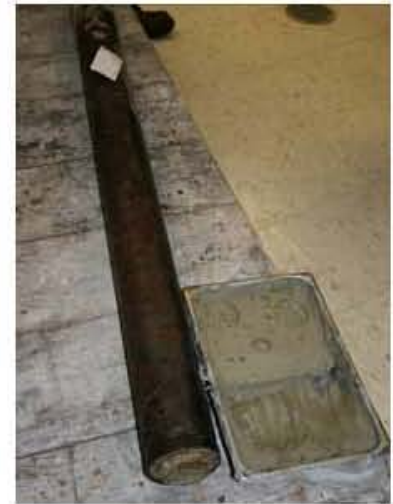

Figure 19. $4 \mathrm{ft}$ Bentonite plug. 
Table 1. Summary of Extrusion Tests with Various Types of Blockages

\begin{tabular}{|c|c|c|c|c|c|c|c|}
\hline Date & Plug Type & Plug length & Max pressure attained & Shear Vane Reading & Penetrometer reading & Video & Notes \\
\hline $10 / 24 / 2008$ & Al-Gel & $4 \mathrm{ft}$ & $5 \mathrm{psi}$ & Top $0.1 \mathrm{rev}$, bottom $0.1 \mathrm{rev}$ & Top 0, bottom 0 & Yes & Plug was too soft for characterization. \\
\hline $10 / 24 / 2008$ & Bentonite $30 \%$ Kaolin30\% & $4 \mathrm{ft}$ & $45 \mathrm{psi}$ & $\begin{array}{l}\text { Top } 1.95,1.95 \text { Bottom } 0.9 \text { and } \\
0.95 \text { rev }\end{array}$ & $\begin{array}{l}\text { Top } 0.5,0.5 \text { and } 0.5 \text { Bottom } \\
0.2,0.2 \text { and } 0.2\end{array}$ & Yes & Volclay BH-40 \\
\hline $10 / 27 / 2008$ & K-Mag 90\% Non-pulverized & $4 \mathrm{ft}$ & $200 \mathrm{psi}$ & N/A & N/A & Yes & $\begin{array}{l}\text { Shear vane and penetrom eter can't penetrate the material. No leaks for } 2.5 \\
\text { minutes with max pressure at } 200 \mathrm{psi} \text {. Pressure dropped to } 50 \mathrm{psi} \text { once water } \\
\text { started leaking. Most of the plug stayed in although some kmag was dissolved } \\
\text { at the tip and was removed f }\end{array}$ \\
\hline $10 / 28 / 2008$ & K-Mag $80 \%$ Non-pulverized & $1 \mathrm{ft}$ & $55 \mathrm{psi}$ & N/A & $\mathrm{N} / \mathrm{A}$ & Yes & Leaks under 55 psi even before pumping is started. \\
\hline $10 / 28 / 2008$ & Phosphate & $4 \mathrm{ft}$ & $30 \mathrm{psi}$ & Top $.6 \mathrm{rev}$ Bottom $.5 \mathrm{rev}$ & $\begin{array}{l}\text { Top } 0.25, .5 \text { and } .5 \text { Bottom } .25, \\
.5 \text { and } .75\end{array}$ & Yes & 8 \\
\hline $10 / 29 / 2008$ & K-Mag 90\% Pulverized & $1 \mathrm{ft}$ & $300 \mathrm{psi}$ & $\mathrm{N} / \mathrm{A}$ & N/A & Yes & $\begin{array}{l}\text { Held } 300 \mathrm{psi} \text { max for } 3-4 \mathrm{mins} \text { then water penetrated thru the sides of the plug } \\
\text { and } 10 \% \text { was removed from the exit. }\end{array}$ \\
\hline $10 / 29 / 2008$ & Bentonite $60 \%$ & $4 \mathrm{ft}$ & $150 \mathrm{psi}$ & $.96 \mathrm{rev}$ & $0.25,0.3$ and 0.3 & Yes & $\begin{array}{l}\text { Volclay BH-40. No leaks until plug starts moving. } 99 \% \text { unplugged as one } \\
\text { chunk. }\end{array}$ \\
\hline $10 / 30 / 2008$ & Bentonite65\% & $4 \mathrm{ft}$ & $190 \mathrm{psi}$ & $1.9 \mathrm{rev}$ & $.5, .6, .6$ & Yes & $\begin{array}{l}\text { Volclay BH-40. No leaks until plug starts moving. 3" of blockage moved out } \\
\text { and the rest stayed in. }\end{array}$ \\
\hline $11 / 3 / 2008$ & K-Mag $90 \%$ Pulverized & $4 \mathrm{ft}$ & $300 \mathrm{psi}$ & $\mathrm{N} / \mathrm{A}$ & $\mathrm{N} / \mathrm{A}$ & No & $\begin{array}{l}\text { Greens Grade. Relief valve leaked above } 290 \text { psi. No leaks for } 20 \mathrm{mins} \text {. } 0 \% \\
\text { unplugged. }\end{array}$ \\
\hline $11 / 3 / 2008$ & Bentonite $60 \%$ non-powderized & $4 \mathrm{ft}$ & $100 \mathrm{psi}$ & & & No & $\begin{array}{l}\text { WyoBend 20. Mixed without pulverizing. No leaks until plug moves. } 99 \% \\
\text { unplugged after } 2 \mathrm{mins} \text {. }\end{array}$ \\
\hline $11 / 5 / 2008$ & Bentonite $60 \%$ non-powderized & $4 \mathrm{ft}$ & $150 \mathrm{psi}$ & $1.15 \mathrm{rev}, 2: 0.95 \mathrm{rev}$ & \begin{tabular}{|l|}
$0.20 .10 .2($ w/out 1 " adapter $)$ \\
$3.753 .75(\mathrm{w} / 1$ " adapter $)$
\end{tabular} & & Wyobend 20 . Plug started getting out at $150 \mathrm{psi}$ \\
\hline $11 / 5 / 2008$ & Benonite $65 \%$ & $4 \mathrm{ft}$ & $185 \mathrm{psi}$ & $1.4 \mathrm{rev}, 2: 1.4 \mathrm{rev}$ & $4.5,4.5$ (w/1"adapter) & & Wyobend- 20 bentonite plug started moving at $185 \mathrm{psi}$ \\
\hline $11 / 5 / 2008$ & $\mathrm{Al} \mathrm{Gel}$ & & $15 \mathrm{psi}$ & $\begin{array}{l}\text { Top: } 0.24 \mathrm{rev}, 0.14 \mathrm{rev} \\
\text { Bottom: } 0.34 \mathrm{rev}, 0.2 \mathrm{rev}\end{array}$ & $\begin{array}{l}\text { Top: } 08,0.75 \mathrm{w} / 1 \text { " Bottom: } \\
1.5,1.25,1.75\end{array}$ & & Blew out at $15 \mathrm{psi}$ \\
\hline $11 / 7 / 2008$ & Bentonite $60 \%$ & $8 \mathrm{ft}$ & $230 \mathrm{psi}$ & (2) & & & Pressure dropped to $170 \mathrm{psiafter}$ applying $230 \mathrm{psi}$ \\
\hline $11 / 10 / 2008$ & Bentonite $68 \%$ & $8 \mathrm{ft}$ & $300 \mathrm{psi}$ & 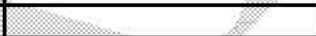 & & & The plug moved $1.75^{\prime \prime}$ after $3 \mathrm{~min}$ but it did not come out completely \\
\hline $11 / 10 / 2008$ & Bentonite $68 \%$ & $4 \mathrm{ft}$ & $100 \mathrm{psi}$ & & & & Pressure dropped to $50 \mathrm{psi}$ and the plug came out in $30 \mathrm{sec}$ \\
\hline $11 / 13 / 2008$ & Bentonite $68 \%$ & $8 \mathrm{ft}$ & $300 \mathrm{psi}$ & & & & Pressure dropped at $300 \mathrm{psi}$ and the plug moved \\
\hline $11 / 13 / 2008$ & Bentonite $68 \%$ & $4 \mathrm{ft}$ & $130 \mathrm{psi}$ & & & & It leaked at $52 \mathrm{psi}$ \\
\hline $11 / 16 / 2008$ & Bentonite $68 \%$ & $4 \mathrm{ft}$ & $170 \mathrm{psi}$ & & & & The entire plug came out. \\
\hline $11 / 18 / 2008$ & Kmag & $4 \mathrm{ft}$ & $650 \mathrm{psi}$ & 2 & & & Held steady. No leaks \\
\hline $11 / 18 / 2008$ & 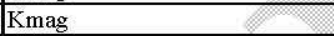 & $4 \mathrm{ft}$ & $600 \mathrm{psi}$ & 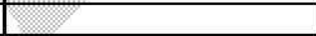 & & & Held $500 \mathrm{psi}$ for $5 \mathrm{~min}$, then $600 \mathrm{psi}$ for an additional $10 \mathrm{~min}$ \\
\hline $11 / 26 / 2008$ & Kmag & $2 \mathrm{ft}$ & $450 \mathrm{psi}$ & (a & & & Started dripping and bulging at $300 \mathrm{psi}$. About 5 " of plug purtrude \\
\hline $12 / 4 / 2008$ & WTP & & $500 \mathrm{psi}$ & & & & Plug prepared on 11/25/08. Plug started coming out and pressure droped. \\
\hline $12 / 8 / 2008$ & Kmag & $4 \mathrm{ft}$ & $300 \mathrm{psi}$ & & & & $\begin{array}{l}\text { First used on blow-out test and then AIMMS. Pipe was not unplugged. } 285 \\
\text { loop used }\end{array}$ \\
\hline
\end{tabular}


Table 2. Chemicals Used to Manufacture $4 \mathrm{ft} \mathrm{Na-AI-Si} \mathrm{Plug}$

\begin{tabular}{|c|c|}
\hline Chemical & Mass $(\mathrm{g})$ \\
\hline $\mathrm{Al}\left(\mathrm{NO}_{3}\right)_{3} 9_{2} \mathrm{O}$ & 28136.27 \\
\hline $\mathrm{NaOH}$ & 6000 \\
\hline $\mathrm{Na}_{2} \mathrm{SiO}_{3}$ & 2287.5 \\
\hline $\mathrm{Na}_{2} \mathrm{SO}_{4}$ & 133.1 \\
\hline $\mathrm{NaF}$ & 15 \\
\hline $\mathrm{NaH}_{2} \mathrm{PO}_{4}$ & 7.5 \\
\hline
\end{tabular}

Phosphate and aluminum based plugs, which were used in previous unplugging experiments were also not qualified for testing with AIMM technologies because of low extrusion pressures (30 and 5 psi respectively). A potassium-magnesium sulfate (Kmag) plug was selected to represent a crystallized salt plug, and a Na-Al-Si plug was selected to emulate a crystallized chemical plug. The recipe for the Na-Al-Si plug was provided from engineers at Pacific Northwest National Laboratory (PNNL). An extrusion test conducted by the engineers showed that the pressure needed to extrude the plug from a $4 \mathrm{ft} 3$ " steel pipe was $150 \mathrm{psi}$. The chemicals used for a Na-Al-Si plug are given in Table 2. The procedure to prepare a $4 \mathrm{ft} \mathrm{Na-Al-Si} \mathrm{plug} \mathrm{is}$ given as:

1) Take a clean plastic bucket.

2) Add the required quantities of Sodium sulphate, sodium silicate, sodium fluoride and sodium phosphate in order. Mix the chemicals by manually shaking the bucket for 30 seconds.

3) Add sodium hydroxide to the above mixture. Make sure that the $\mathrm{NaOH}$ is weighed just before addition, so that the amount of water it gains from the atmosphere is minimal. Also all the chemical must be covered with air tight lids.

4) Once again manually shake the bucket so that $\mathrm{NaOH}$ gets mixed thoroughly and coated with other chemicals.

5) Add Na-Al-Si to the mix. Use an automatic drill with a long mixer extension to mix aluminum nitrate with the earlier chemicals mixture. Keep on stirring the entire mixture for about 2 minutes until you see the white fumes coming out. At this point you will not be able to see the bottom of the bucket clearly due to the fumes. Also the mixture should have turned into a gel like solution. It is recommended that this step is done in a walk-in fume hood.

6) Let the white fumes clear. This should take about 15-20 seconds. Once cleared, you can resume stirring for about 1 minute. The gel like mixture turns into liquid. You also see the white fumes coming out again.

7) Stop mixing and let the white fumes clear out. Keep the bucket still for 1 minute. The solution is very hot, so make sure you have the rubber gloves on top of the latex gloves. 
8) Tilt the bucket sideways and scoop the gel like mixture into the pipes and leave the solution in the pipe overnight to solidify.

Since the extrusion pressure of the plug is low, engineers from PNNL suggested an alternative procedure for developing a plug that has stronger mechanical properties and is more likely to occur. The alternative approach is to keep mixing at the point (in line 7) where the recipe indicates to stop. This creates a semi-solid precipitate, and the liquid mixture and the solid can be removed and placed into the $4 \mathrm{ft}$ pipe. After a short period of time the semi-solid precipitate settles and forms a hard crystallized material and the remaining liquid is decanted off. Figure 20 shows a crystallized sample of the Na-Al-Si prepared in a bucket.

The Kmag plug was selected due to its high mechanical strength. The product comes in a granular form and had to be pulverized using a grinding machine before mixing with water. For a $4 \mathrm{ft}$ plug containing $90 \%$ of Kmag and $10 \%$ water mixture, 1.5 liters of water was mixed with $13.5 \mathrm{~kg}$ of Kmag in a bucket using a drill mixer for about 30 minutes. The mixture was then poured into a $4 \mathrm{ft}$ steel pipe that was closed on one side using a Victaulic cap and a Victaulic 77 coupling. The material is packed using a plunger during filling. When the pipe was full the open end is sealed using parafilm and the pipe is left to cure overnight. The final product is shown in Figure 21.

All of the plugs were created in 4-foot pipe lengths except Kmag plug which was manufactured in 2-ft and 4-ft pipes. They were weighed before and after each unplugging test to determine the weight of removed plug material and effective unplugging rates.

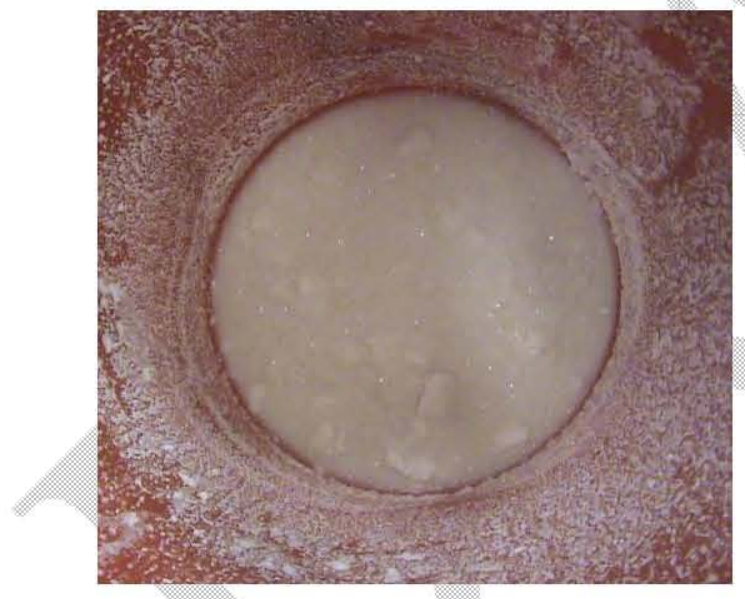

Figure 20. Na-Al-Si plug prepared in bucket.

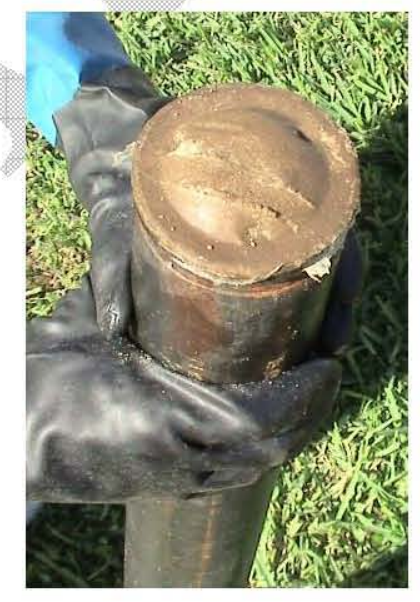

Figure 21. 4-ft Kmag plug.

\section{AIMM TECHNOLOGIES EQUIPMENT}

The Hydrokinetics system comprises of a water tank, plunger pump, air compressor and a control unit. Water was stored in a 2500-gallon storage system (Figure 22) and was fed to the plunger pump shown in Figure 23 during the unplugging operation. An air compressor, shown in Figure 24, provides compressed air at $75 \mathrm{psi}$. The compressed air is connected to the control unit (Figure 25 ) in order to operate the pneumatic valves that control the flow in and out of the control unit. The gauge on the left hand side in Figure 25 is used to track the water pressure in the control unit and the regulating valve at the top is used to adjust the pressure in the pipeline. The switches on 
the left and right side are used to let water in and out the control unit and create pulses. The frequencies of pulsations are determined manually by the operator.

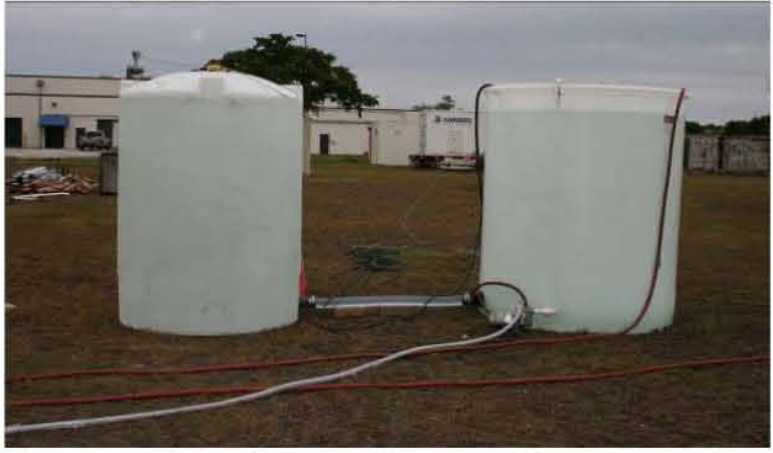

Figure 22. 1000-gallon and 1500-gallon water tanks.

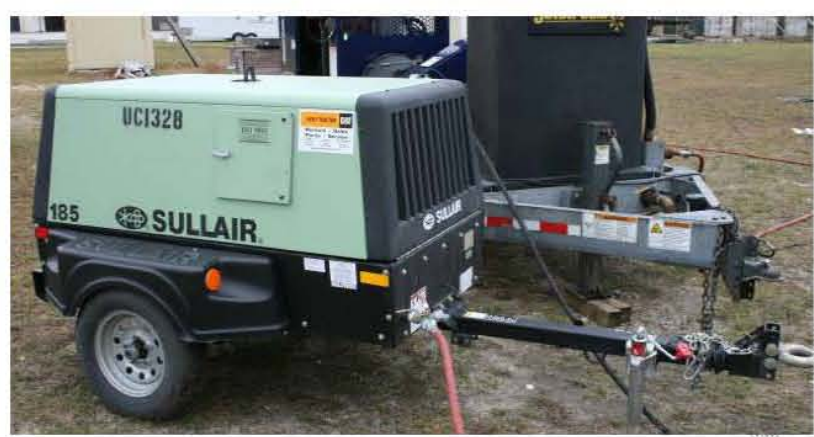

Figure 24. Mobile air compressor.

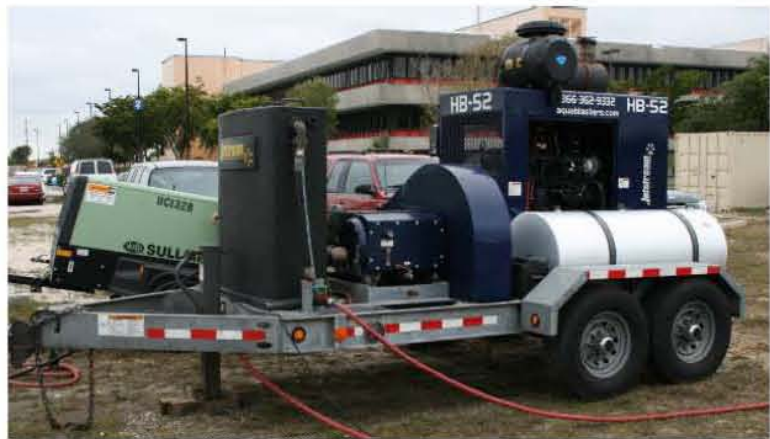

Figure 23. Aquablasters plunger pump.

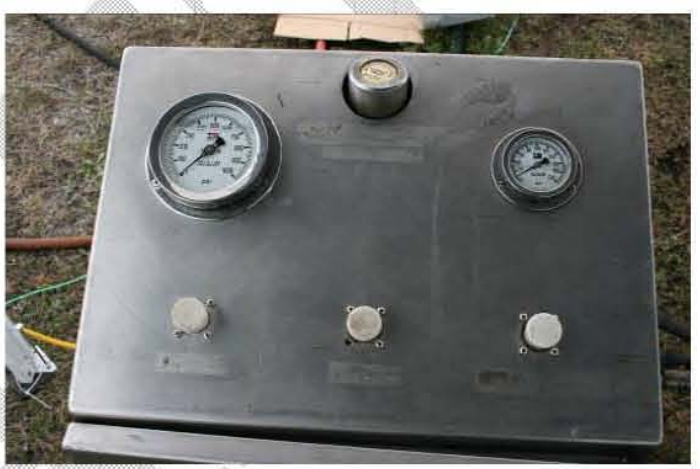

Figure 25. Hydrokinetics control unit.

\section{TEST PLAN}

A total of 26 different unplugging trials have been completed with AIMM's unplugging technology. During 12 of these cases, trials were run for commission purposes with partial data collected (Table 3 ). The remaining 15 cases correspond to the final test plan where data was collected (Table 4). Cases with data include seven from the $310 \mathrm{ft}$ pipeline, five from the $646 \mathrm{ft}$ pipeline and five from the $1822 \mathrm{ft}$ pipeline. For the cases with no data, only the unplugging times, unplugging efficiencies and maximum pressures were recorded.

Three different blockages were used on each of the three pipelines. The blockages were placed into 4-ft carbon steel sections which were combined together using couplings for the Bentonite 8 $\mathrm{ft}$ and $12 \mathrm{ft}$ cases. A $21 \mathrm{ft}$ discharge section was placed after the blockage section which was connected using a $90^{\circ}$ Victaulic elbow. The blockage had to make its way around the elbow before getting unplugged.

The final test plan also shows that an expansion joint was used in the baseline test bed for all plug types and all pipe lengths. (The number 1 in the test plan indicates that it was used in the trial and the number 0 indicates that it was not used in the trial.) For the $310 \mathrm{ft}$ test bed, one trial was conducted without the expansion joint using a Na-Al-Si blockage to determine the effects of the expansion joint. For the 310 -ft test bed, a constriction was inserted using two 3" to 2" reducers just upstream of the expansion joint to see what effects it may have on the pipeline pressures during testing with the capped pipeline. Capped pipeline testing (no plug) was 
conducted in which the control unit pressures and pulsation frequencies were varied to analyze their effects on the pulsation mechanics and resulting pressures. Table 5 through Table 8 show the variation in control variables for the parametric testing at each of the three pipeline test bed lengths.

Table 3. Initial Commissioning Test Cases

\begin{tabular}{|cccccc|}
\hline Trial \# & Reducer & $\begin{array}{c}\text { Expansion } \\
\text { Joint }\end{array}$ & $\begin{array}{c}\text { Blockage } \\
\text { Type }\end{array}$ & $\begin{array}{c}\text { Blockage } \\
\text { length }\end{array}$ & $\begin{array}{c}\text { Distance to } \\
\text { Blockage }\end{array}$ \\
\hline 1 & 0 & 1 & Kmag & $2 \mathrm{ft}$ & $310 \mathrm{ft}$ \\
2 & 0 & 1 & Kmag & $2 \mathrm{ft}$ & $310 \mathrm{ft}$ \\
3 & 0 & 1 & Kmag & $2 \mathrm{ft}$ & $310 \mathrm{ft}$ \\
4 & 0 & 1 & Kmag & $4 \mathrm{ft}$ & $310 \mathrm{ft}$ \\
5 & 0 & 1 & Bentonite & $4 \mathrm{ft}$ & $310 \mathrm{ft}$ \\
6 & 0 & 1 & Bentonite & $4 \mathrm{ft}$ & $310 \mathrm{ft}$ \\
\hline 7 & 0 & 1 & Bentonite & $4 \mathrm{ft}$ & $646 \mathrm{ft}$ \\
8 & 0 & 1 & Bentonite & $8 \mathrm{ft}$ & $646 \mathrm{ft}$ \\
9 & 0 & 1 & Bentonite & $8 \mathrm{ft}$ & $646 \mathrm{ft}$ \\
10 & 0 & 1 & Na-Al-Si & $4 \mathrm{ft}$ & $646 \mathrm{ft}$ \\
11 & 0 & 1 & Kmag & $2 \mathrm{ft}$ & $646 \mathrm{ft}$ \\
\hline 12 & 0 & 1 & Bentonite & $8 \mathrm{ft}$ & $1822 \mathrm{ft}$ \\
\hline
\end{tabular}

Table 4. Final Test Plan

\begin{tabular}{|cccccc|}
\hline Trial \# & Reducer & $\begin{array}{c}\text { Expansion } \\
\text { Joint }\end{array}$ & $\begin{array}{c}\text { Blockage } \\
\text { Type }\end{array}$ & $\begin{array}{c}\text { Blockage } \\
\text { length }\end{array}$ & $\begin{array}{c}\text { Distance to } \\
\text { Blockage }\end{array}$ \\
\hline 1 & 0 & 1 & Bentonite & $8 \mathrm{ft}$ & $310 \mathrm{ft}$ \\
2 & 0 & 1 & Bentonite & $12 \mathrm{ft}$ & $310 \mathrm{ft}$ \\
3 & 0 & 1 & Kmag & $4 \mathrm{ft}$ & $310 \mathrm{ft}$ \\
4 & 0 & 1 & Na-Al-Si & $4 \mathrm{ft}$ & $310 \mathrm{ft}$ \\
5 & 0 & 0 & Na-Al-Si & $4 \mathrm{ft}$ & $310 \mathrm{ft}$ \\
6 & 0 & 1 & Capped & N/A & $310 \mathrm{ft}$ \\
7 & 1 & 1 & Capped & N/A & $310 \mathrm{ft}$ \\
\hline 8 & 0 & 1 & Bentonite & $8 \mathrm{ft}$ & $646 \mathrm{ft}$ \\
9 & 0 & 1 & Bentonite & $12 \mathrm{ft}$ & $646 \mathrm{ft}$ \\
10 & 0 & 1 & Kmag & $4 \mathrm{ft}$ & $646 \mathrm{ft}$ \\
11 & 0 & 1 & Na-Al-Si & $4 \mathrm{ft}$ & $646 \mathrm{ft}$ \\
12 & 0 & 1 & Capped & N/A & $646 \mathrm{ft}$ \\
\hline
\end{tabular}




\begin{tabular}{|lllccc|}
\hline 13 & 0 & 1 & Bentonite & $8 \mathrm{ft}$ & $1822 \mathrm{ft}$ \\
14 & 0 & 1 & Bentonite & $12 \mathrm{ft}$ & $1822 \mathrm{ft}$ \\
15 & 0 & 1 & Kmag & $4 \mathrm{ft}$ & $1822 \mathrm{ft}$ \\
16 & 0 & 1 & Na-Al-Si & $4 \mathrm{ft}$ & $1822 \mathrm{ft}$ \\
17 & 0 & 1 & Capped & N/A & $1822 \mathrm{ft}$ \\
18 & 0 & 0 & Capped & N/A & $1822 \mathrm{ft}$ \\
\hline
\end{tabular}

Table 5. Parametric Test Plan at $\mathbf{3 1 0} \mathrm{ft}$ with Reducer not Connected

\begin{tabular}{|ccccc|}
\hline Trial \# & Pipeline Length & Pressure & Pump RPM & Expansion Joint \\
1 & $310 \mathrm{ft}$ & $250 \mathrm{psi}$ & 1350 & 1 \\
2 & $310 \mathrm{ft}$ & $250 \mathrm{psi}$ & 1410 & 1 \\
3 & $310 \mathrm{ft}$ & $250 \mathrm{psi}$ & 1450 & 1 \\
4 & $310 \mathrm{ft}$ & $200 \mathrm{psi}$ & 1350 & 1 \\
5 & $310 \mathrm{ft}$ & $200 \mathrm{psi}$ & 1410 & 1 \\
6 & $310 \mathrm{ft}$ & $200 \mathrm{psi}$ & 1450 & 1 \\
7 & $310 \mathrm{ft}$ & $150 \mathrm{psi}$ & 1350 & 1 \\
8 & $310 \mathrm{ft}$ & $150 \mathrm{psi}$ & 1410 & 1 \\
9 & $310 \mathrm{ft}$ & $150 \mathrm{psi}$ & 1450 & 1 \\
\hline
\end{tabular}

Table 6. Parametric Test Plan at $\mathbf{3 1 0} \mathrm{ft}$ with Reducer Connected

\begin{tabular}{|ccccc|}
\hline Trial \# & Pipeline Length & Pressure & Pump RPM & Expansion Joint \\
1 & $310 \mathrm{ft}$ & $200 \mathrm{psi}$ & 1350 & 1 \\
2 & $310 \mathrm{ft}$ & $200 \mathrm{psi}$ & 1410 & 1 \\
3 & $310 \mathrm{ft}$ & $250 \mathrm{psi}$ & 1450 & 1 \\
\hline
\end{tabular}


Table 7. Parametric Test Plan at $646 \mathrm{ft}$

\begin{tabular}{|ccccc|}
\hline Trial \# & Pipeline Length & Pressure & Pump RPM & Expansion Joint \\
1 & $646 \mathrm{ft}$ & $250 \mathrm{psi}$ & 1350 & 1 \\
2 & $646 \mathrm{ft}$ & $250 \mathrm{psi}$ & 1410 & 1 \\
3 & $646 \mathrm{ft}$ & $250 \mathrm{psi}$ & 1450 & 1 \\
4 & $646 \mathrm{ft}$ & $200 \mathrm{psi}$ & 1350 & 1 \\
5 & $646 \mathrm{ft}$ & $200 \mathrm{psi}$ & 1410 & 1 \\
6 & $646 \mathrm{ft}$ & $200 \mathrm{psi}$ & 1450 & 1 \\
7 & $646 \mathrm{ft}$ & $150 \mathrm{psi}$ & 1350 & 1 \\
8 & $646 \mathrm{ft}$ & $150 \mathrm{psi}$ & 1410 & 1 \\
9 & $646 \mathrm{ft}$ & $150 \mathrm{psi}$ & 1450 & 1 \\
\hline
\end{tabular}

Table 8. Parametric Test Plan at $1822 \mathrm{ft}$

\begin{tabular}{|c|c|c|c|c|}
\hline Trial \# & Pipeline Length & Pressure & Pump RPM & Expansion Joint \\
\hline 1 & $1822 \mathrm{ft}$ & $250 \mathrm{psi}$ & 1350 & 1 \\
\hline 2 & $1822 \mathrm{ft}$ & $250 \mathrm{psi}$ & $1402-1416$ & 1 \\
\hline 3 & $1822 \mathrm{ft}$ & $250 \mathrm{psi}$ & 1450 & 1 \\
\hline 4 & $1822 \mathrm{ft}$ & $200 \mathrm{psi}$ & 1350 & 1 \\
\hline 5 & $1822 \mathrm{ft}$ & $200 \mathrm{psi}$ & $1407-1412$ & 1 \\
\hline 6 & $1822 \mathrm{ft}$ & $200 \mathrm{psi}$ & 1450 & 1 \\
\hline 7 & $1822 \mathrm{ft}$ & 150 psi & 1350 & 1 \\
\hline 8 & $1822 \mathrm{ft}$ & $150 \mathrm{psi}$ & 1410 & 1 \\
\hline 9 & $1822 \mathrm{ft}$ & $150 \mathrm{psi}$ & 1450 & 1 \\
\hline 10 & $1822 \mathrm{ft}$ & $250 \mathrm{psi}$ & 1350 & 0 \\
\hline 11 & $1822 \mathrm{ft}$ & $250 \mathrm{psi}$ & $1407-1410$ & 0 \\
\hline 12 & $1822 \mathrm{ft}$ & $250 \mathrm{psi}$ & 1450 & 0 \\
\hline 13 & $1822 \mathrm{ft}$ & $200 \mathrm{psi}$ & 1350 & 0 \\
\hline 14 & $1822 \mathrm{ft}$ & $200 \mathrm{psi}$ & 1410 & 0 \\
\hline 15 & $1822 \mathrm{ft}$ & $200 \mathrm{psi}$ & 1450 & 0 \\
\hline 16 & $1822 \mathrm{ft}$ & $150 \mathrm{psi}$ & 1350 & 0 \\
\hline 17 & $1822 \mathrm{ft}$ & $150 \mathrm{psi}$ & 1413 & 0 \\
\hline 18 & $1822 \mathrm{ft}$ & $150 \mathrm{psi}$ & 1450 & 0 \\
\hline
\end{tabular}


The summary of unplugging cases tested with AIMM Technologies is shown in Table 9. AIMM Technologies was able to unplug 6 out of 13 cases tested at three different pipeline lengths. The shortest unplugging time was $16 \mathrm{~min}$, while the longest unplugging time was $73 \mathrm{~min}$. In all of the cases, the technology was kept from pressurizing the pipeline above 300 psi.

Table 9. Summary of Unplugging Cases in the Final Test Plan

\begin{tabular}{|c|c|c|c|c|c|c|c|}
\hline $\begin{array}{c}\text { Trial } \\
\#\end{array}$ & $\begin{array}{c}\text { Expansion } \\
\text { Joint }\end{array}$ & $\begin{array}{c}\text { Distance } \\
\text { to } \\
\text { Blockage }\end{array}$ & $\begin{array}{c}\text { Blockage } \\
\text { Type }\end{array}$ & $\begin{array}{c}\text { Blockage } \\
\text { length }\end{array}$ & $\begin{array}{c}\text { Maximum } \\
\text { Pressure }\end{array}$ & Success & Time \\
\hline 1 & 1 & $310 \mathrm{ft}$ & Bentonite & $8 \mathrm{ft}$ & $272.2 \mathrm{psi}$ & Yes & $16 \mathrm{~min}$ \\
\hline 2 & 1 & $310 \mathrm{ft}$ & Bentonite & $12 \mathrm{ft}$ & $286.3 \mathrm{psi}$ & Yes & $30 \mathrm{~min}$ \\
\hline 3 & 1 & $310 \mathrm{ft}$ & Kmag & $4 \mathrm{ft}$ & $265.1 \mathrm{psi}$ & Partial & $40 \mathrm{~min}$ \\
\hline 4 & 1 & $310 \mathrm{ft}$ & Na-Al-Si & $4 \mathrm{ft}$ & $292.8 \mathrm{psi}$ & No & $52 \mathrm{~min}$ \\
\hline 5 & 0 & $310 \mathrm{ft}$ & Na-Al-Si & $4 \mathrm{ft}$ & $285 \mathrm{psi}$ & No & $21 \mathrm{~min}$ \\
\hline 6 & 1 & $646 \mathrm{ft}$ & Bentonite & $8 \mathrm{ft}$ & $225.3 \mathrm{psi}$ & Yes & $17 \mathrm{~min}$ \\
\hline 7 & 1 & $646 \mathrm{ft}$ & Bentonite & $12 \mathrm{ft}$ & $264.2 \mathrm{psi}$ & Yes & $18 \mathrm{~min}$ \\
\hline 8 & 1 & $646 \mathrm{ft}$ & Kmag & $4 \mathrm{ft}$ & $287.1 \mathrm{psi}$ & No & $54 \mathrm{~min}$ \\
\hline 9 & 1 & $646 \mathrm{ft}$ & Na-Al-Si & $4 \mathrm{ft}$ & $278.3 \mathrm{psi}$ & No & $41 \mathrm{~min}$ \\
\hline 10 & 1 & $1822 \mathrm{ft}$ & Bentonite & $8 \mathrm{ft}$ & $291.7 \mathrm{psi}$ & No & $115 \mathrm{~min}$ \\
\hline 11 & 1 & $1822 \mathrm{ft}$ & Bentonite & $12 \mathrm{ft}$ & $214.2 \mathrm{psi}$ & Yes & $73 \mathrm{~min}$ \\
\hline 12 & 1 & $1822 \mathrm{ft}$ & Kmag & $4 \mathrm{ft}$ & $318.7 \mathrm{psi}$ & No & $40 \mathrm{~min}$ \\
\hline 13 & 1 & $1822 \mathrm{ft}$ & Na-Al-Si & $4 \mathrm{ft}$ & $286.8 \mathrm{psi}$ & No & $52 \mathrm{~min}$ \\
\hline
\end{tabular}

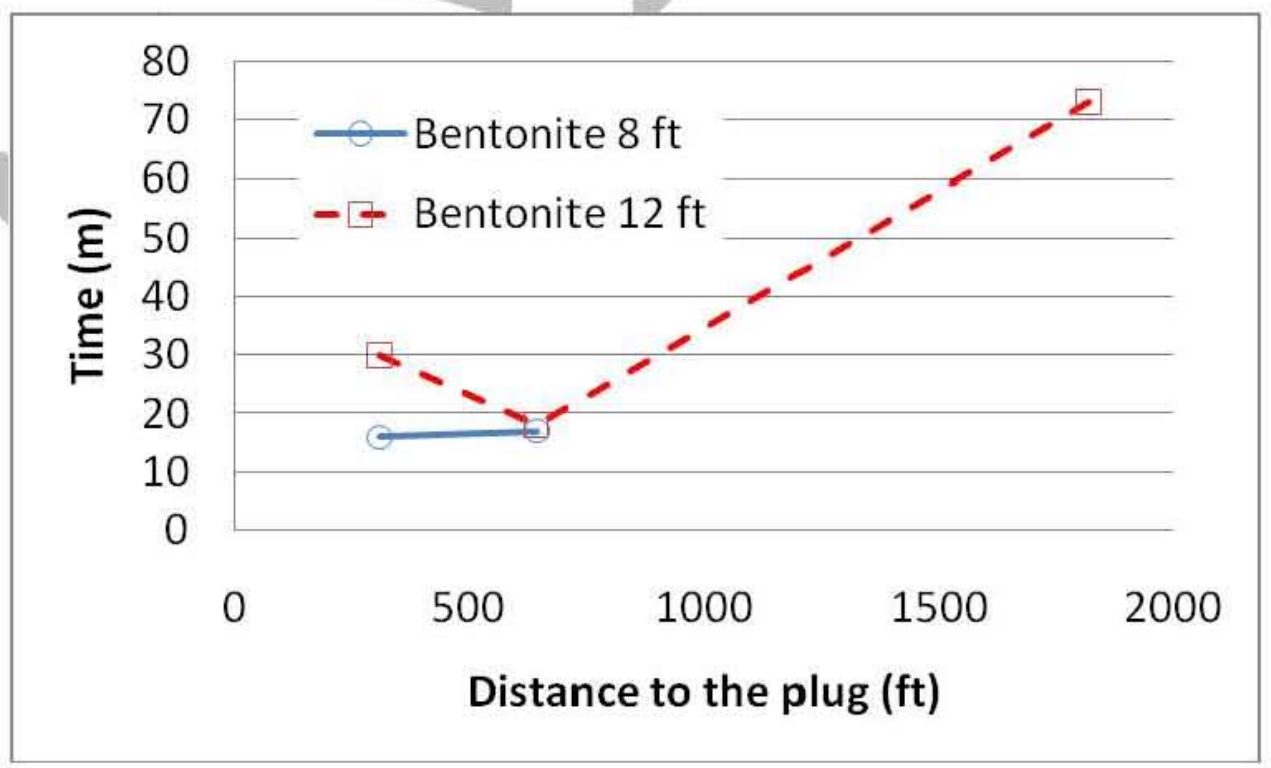

Figure 26. Total time required to unplug Bentonite plugs. 
Figure 26 shows that the time required to unplug Bentonite plugs generally demonstrated an increasing trend for both $8 \mathrm{ft}$ and $12 \mathrm{ft}$ plugs as the distance to the plug is increased from $310 \mathrm{ft}$ to $1822 \mathrm{ft}$. The increase in time is primarily associated to the time it takes to fill the pipeline in order to build the pressure inside the pipeline to the desired level.

\section{FT PIPELINE TRIALS}

During the commissioning trials at the $310 \mathrm{ft}$ pipeline, two $4 \mathrm{ft}$ Bentonite plugs were successfully removed without requiring pulsations at 80 and 150 psi maximum pressures, respectively. Therefore, during the final test plan only $8 \mathrm{ft}$ and $12 \mathrm{ft}$ Bentonite plugs were used. Three $2 \mathrm{ft}$ Kmag plugs were partially unplugged at $60 \mathrm{psi}, 100 \mathrm{psi}$ and $280 \mathrm{psi}$ maximum pressures. In one of the cases with $2 \mathrm{ft}$ Kmag plugs, 6 inches of plug was pushed out of the pipeline (Figure 29) while in the other Kmag cases, large gaps were created at the plugs through which water was able to flow (Figure 30). However there has been one case during the commissioning trials where the technology was unsuccessful in unplugging a $4 \mathrm{ft}$ Kmag plug while maintaining pulsations at 305 psi for 1 hour and 15 minutes. Since two of the $2 \mathrm{ft}$ Kmag plugs were removed at low pressures, only $4 \mathrm{ft} \mathrm{Kmag} \mathrm{plugs} \mathrm{were} \mathrm{utilized} \mathrm{for} \mathrm{the} \mathrm{final} \mathrm{test} \mathrm{plan} \mathrm{trials.}$

During the final test plan trials for the $310 \mathrm{ft}$ pipeline, AIMM Technologies was successful in unplugging an $8 \mathrm{ft}$ Bentonite plug at 272.2 psi maximum pressure (Figure 27), a $12 \mathrm{ft}$ Bentonite plug at $286.3 \mathrm{psi}$ (Figure 28) during the trials for final test plan. AIMM Technologies was able to partially unplug a $4 \mathrm{ft} \mathrm{Kmag} \mathrm{plug} \mathrm{at} 265.1$ psi. AIMM Technologies was also not able to unplug two $4 \mathrm{ft} \mathrm{Na-Al-Si} \mathrm{plugs} \mathrm{using} \mathrm{pulsations} \mathrm{at} 300$ psi and 285 psi maximum pressures.
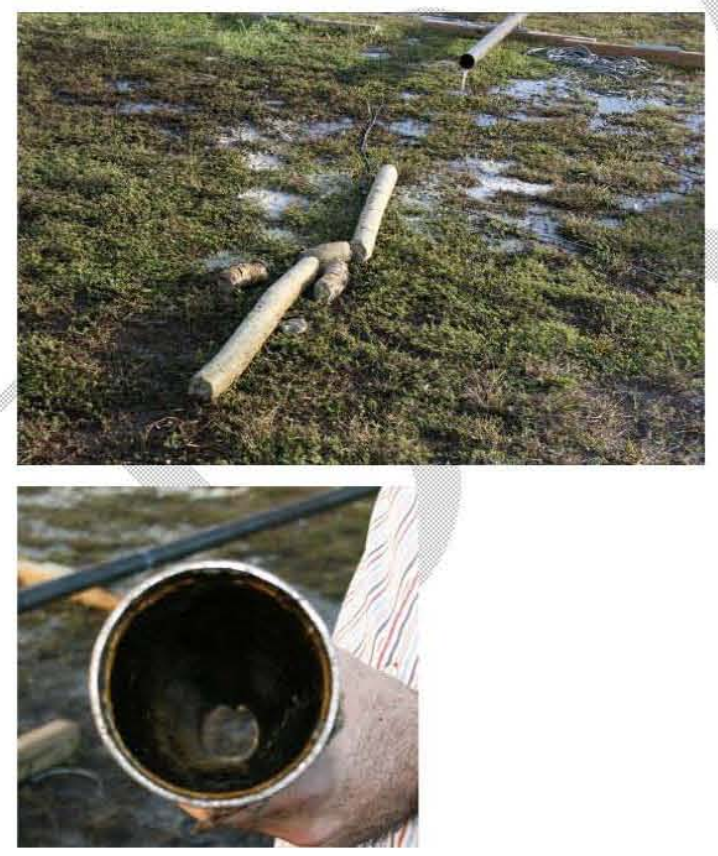

Figure 27. Unplugged $8 \mathrm{ft}$ Bentonite plug at $310 \mathrm{ft}$ pipeline (top). Unplugged blockage section (bottom).

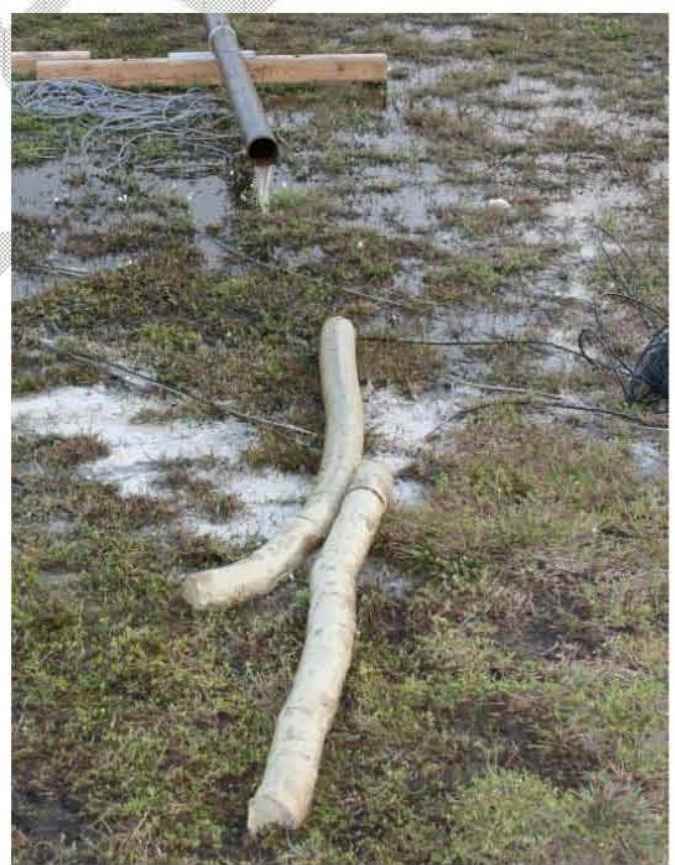

Figure 28. Unplugged $12 \mathrm{ft}$ Bentonite plug (310 ft pipeline). 


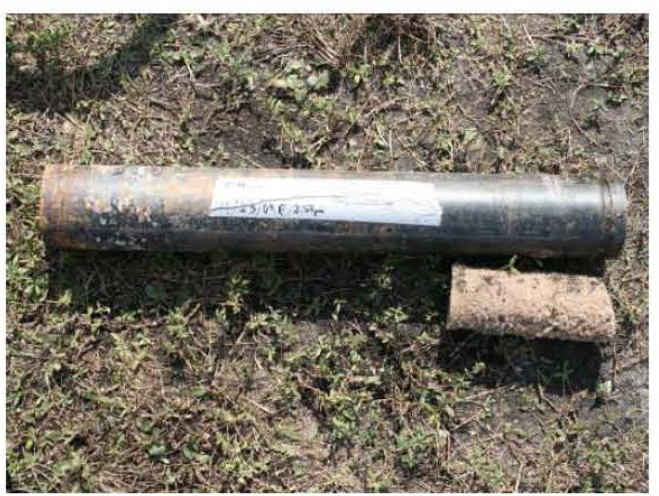

Figure 29. 6 in of Kmag unplugged from $2 \mathrm{ft}$ blockage section.

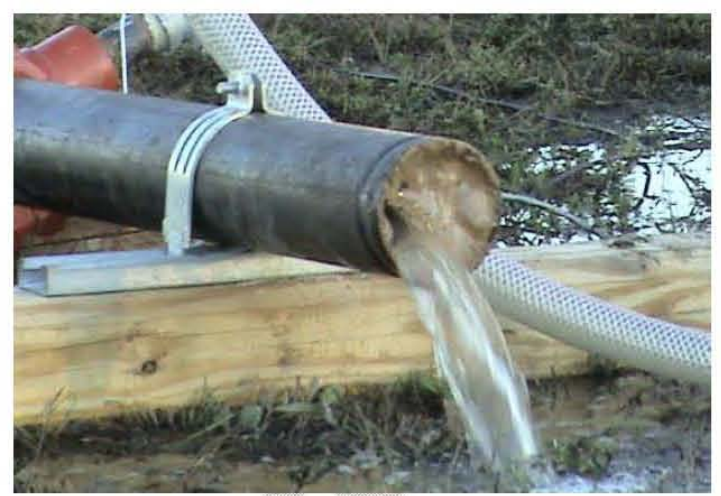

Figure 30. Flow achieved through $2 \mathrm{ft} \mathrm{Kmag}$ plug.

\section{FT PIPELINE TRIALS}

During the commissioning trials at the $646 \mathrm{ft}$ pipeline, AIMM Technologies was able to unplug a $4 \mathrm{ft}$ Bentonite plug at $200 \mathrm{psi}$, an $8 \mathrm{ft}$ Bentonite plug at $160 \mathrm{psi}$, one $2 \mathrm{ft} \mathrm{Kmag}$ plug at $285 \mathrm{psi}$ and one $4 \mathrm{ft} \mathrm{Na-Al-Si} \mathrm{by} \mathrm{doing} \mathrm{pulsations} \mathrm{at} 295 \mathrm{psi}$ respectively. There was one case where an 8 $\mathrm{ft}$ Bentonite plug was not unplugged after pulsating at $260 \mathrm{psi}$. The plug material in the pipe was observed to be compressed $2.8 \mathrm{~cm}$.

During the trials in the final test plan at $646 \mathrm{ft}$ pipeline, one $8 \mathrm{ft}$ Bentonite plug at $225.3 \mathrm{psi}$ and one $12 \mathrm{ft}$ Bentonite plug at 264.2 psi pressure were unplugged. AIMM Technologies was not successful in unplugging a $4 \mathrm{ft}$ Kmag plug and one $4 \mathrm{ft} \mathrm{Na-Al-Si}$ by using Hydrokinetics pulsations at $280 \mathrm{psi}$ for $55 \mathrm{~min}$ and $285 \mathrm{psi}$ for $40 \mathrm{~min}$, respectively.

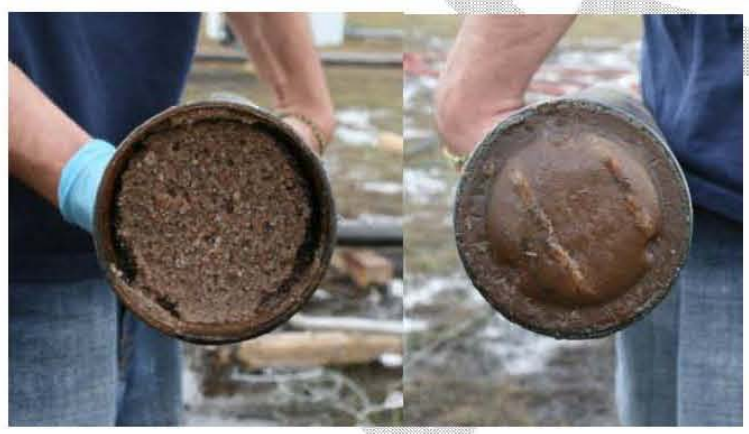

Figure 31. $4 \mathrm{ft}$ Kmag plug after unplugging trials with AIMM. Front side (left) and back side (right).

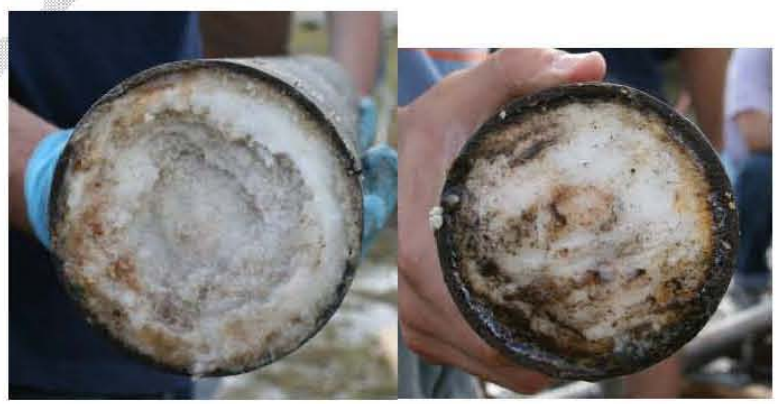

Figure 32. $4 \mathrm{ft} \mathrm{Na-Al-Si} \mathrm{plug} \mathrm{after} \mathrm{unplugging} \mathrm{trials}$ with AIMM. Front side (left) and back side (right). 


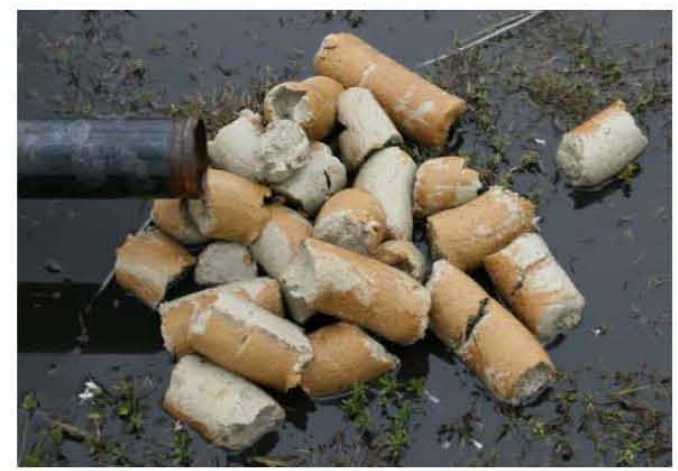

Figure 33. Unplugged $12 \mathrm{ft}$ Bentonite plug after trials with $\mathrm{AIMM}$ at $1822 \mathrm{ft}$ pipeline.

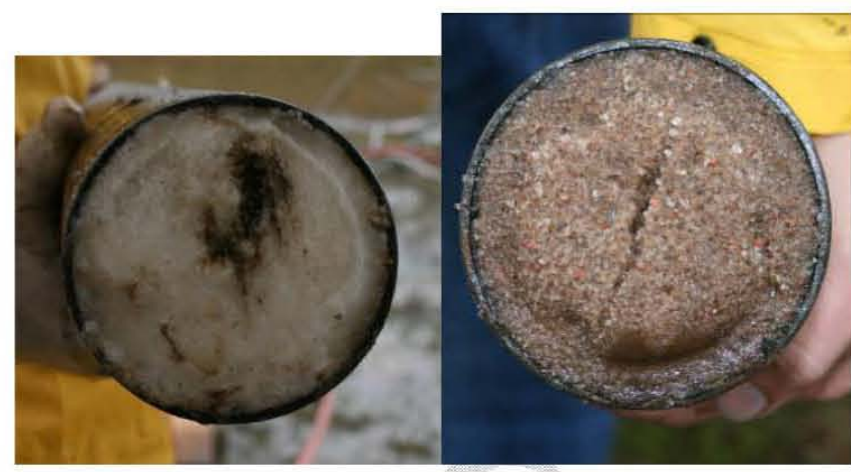

Figure 34. $4 \mathrm{ft} \mathrm{Na-Al-Si} \mathrm{plug} \mathrm{(left)} \mathrm{and} \mathrm{Kmag} \mathrm{plug} \mathrm{(right)}$ after unplugging trials with AIMM.

\section{FT PIPELINE TRIALS}

During the commissioning trials at the $1822 \mathrm{ft}$ pipeline, only one $8 \mathrm{ft}$ Bentonite plug was tested and AIMM Technologies was unsuccessful after pulsing at 285 psi for 80 minutes.

During the final test plan trials, one $12 \mathrm{ft}$ Bentonite plug was unplugged (Figure 32) at a pressure of $214.2 \mathrm{psi}$. However, one $8 \mathrm{ft}$ Bentonite plug was not unplugged at $291.7 \mathrm{psi}$ pulsing pressure for 2 hours. In addition, one $4 \mathrm{ft} \mathrm{Kmag} \mathrm{plug} \mathrm{and} \mathrm{one} 4 \mathrm{ft} \mathrm{Na-Al-Si} \mathrm{were} \mathrm{not} \mathrm{unplugged} \mathrm{by}$ conducting pulsations, which peeked the maximum pressure at $318.7 \mathrm{psi}$ for $40 \mathrm{~min}$ and at 286.8 psi for $52 \mathrm{~min}$, respectively (Figure 33).

\section{DATA ANALYSIS}

In order to analyze the data that is collected during the unplugging trials with AIMM Technologies, a LabView Virtual Interface (VI) has been developed. The Labview VI enabled observation of changes in pressure, temperature and acceleration profiles simultaneously with the operation of the Hydrokinetics method. The VI was used to store the changes in pressure and pipeline wall acceleration during the unplugging process. The collected data was used to investigate the effect of pipeline length and changes in geometry on the propagation of pressure from the inlet point to the plug location.

All successful trials share a common trend on the pressure vs. time plot regardless of the length of the test bed used. Figure 36 shows such a trend for pressure variations in the $310 \mathrm{ft}$ pipeline where an $8 \mathrm{ft}$ Bentonite plug was used. The red signal in Figure 36 refers to the pressure values recorded at the inlet $(\mathrm{P} 1)$ and the green signal refers to the pressure values recorded at the second pressure transducer (P2) (Figure 1). In the temporal variations of pressure, two separate zones can be identified: a flooding zone and target pressure zone. The flooding zone includes the pressurization of the pipeline from atmospheric pressure to the target pressure.

The target pressure zone refers to the region where the pressure in the pipeline has equaled the pressure in the AIMM control box. AIMM operators start creating pulsations usually after achieving the target pressure but on some trials, pressure pulses were generated during the flooding. The time it takes to reach the target pressure zone depends on the volume of the pipeline to be filled and increases with the total distance to the plug (Figure 26). 


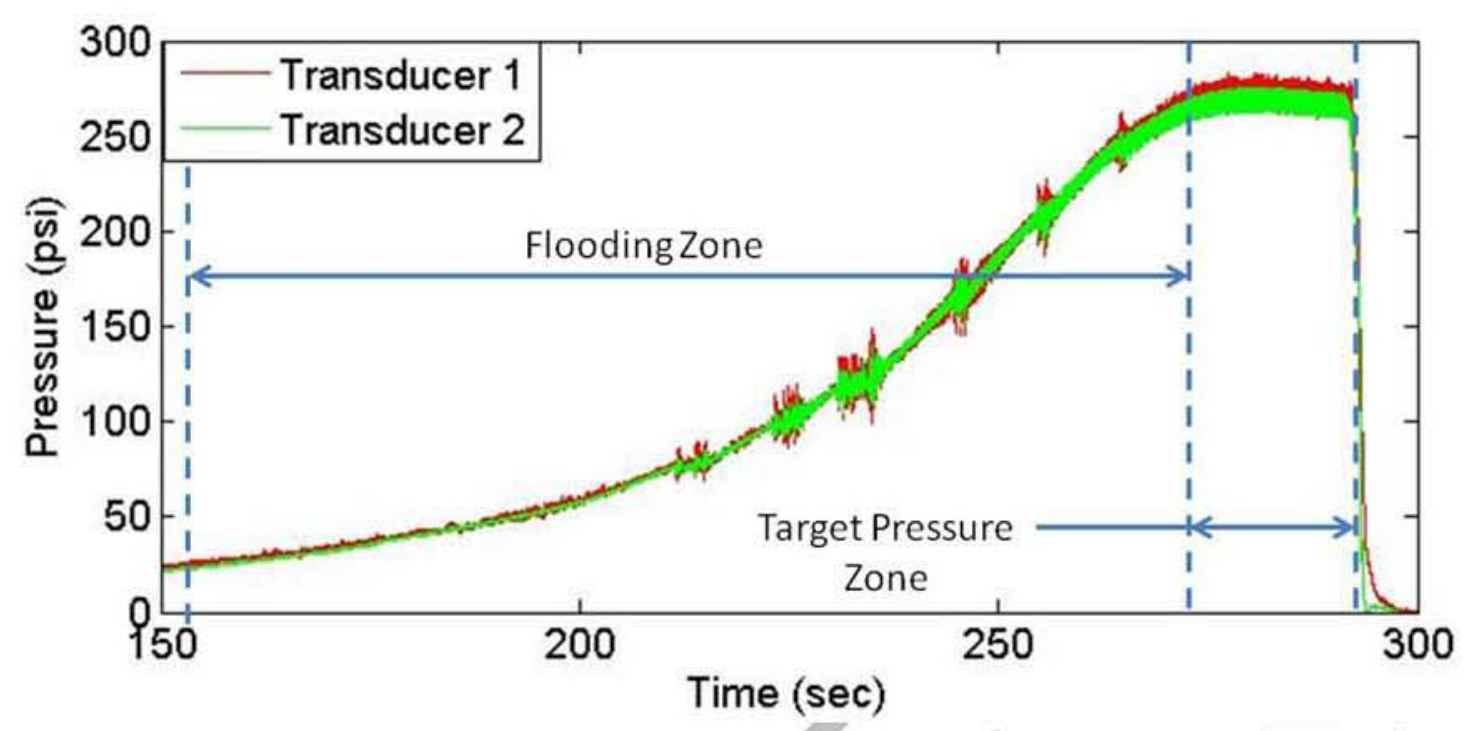

Figure 35. Typical pressure curve of a successful umplugging trial

The analysis of the data collected during the experiments with AIMM Technologies aims to identify the following:

1) Effects of pipeline length and geometry on pipeline pressure distribution and vibration at the plug section.
a. Effect of distance to the plug
b. Effect of elbows
c. Effect of expansion joint
d. Effect of reducer

2) Major frequencies in the pipeline caused by the pulsations at the inlet.

The analysis of the items listed above has been conducted for both the flooding zone and the target pressure zone. However, due to the difference in the nature of the data collected in these two regions, the method used in the analysis has been varied for the different zones. The details of the methods used for each zone is explained below.

As discussed above, AIMM Technologies start creating pulsations at the control unit once the pressure in the pipeline has reached the target pressure zone. The target pressure zone limit was usually set to $250-280$ psi. Once this pressure level was achieved in the system then the frequent pulsations were started and they resulted in very rapid fluctuations around the target pressure value in the pipeline, which helped in breaking the bonds holding the blockage in the pipes. These fluctuations were difficult to detect, therefore a more general calculation using the average values was preferred for the analysis in the target pressure zone. Additionally, for some cases unplugging occurred before the target zone was reached. In these cases, a short region before unplugging occurs was approximated as the target pressure zone.

However, there were cases where AIMM Technologies also randomly created pressure pulses in the flooding zone as shown in Figure 35. These pulsations were less frequent compared to the pulsations in the target pressure zone; therefore it was possible to isolate the effect of each 
pulsation in the pipeline. In the flooding zone the effects of individual pulsations were detected and analyzed separately. The start and end of each pulse or "region of disturbance" was determined by calculating the deviation of pressure from the moving average at the inlet (P1). The criterion to identify the starting point of a disturbance in the flooding zone is given below:

\section{$\left|P-P_{\text {mann }}\right|>$ Sensitivity + Scale $\times P_{\text {mann }}$}

Sensitivity $(0.5-0.7)$ and scale $(0.1-0.5)$ factors were adjusted in order to capture the disturbances accurately. Using this criterion, the disturbances created by each pulsation during the flooding zone was extracted.

\section{EFFECT OF PIPELINE LENGTH AND GEOMETRY ON PRESSURE}

\section{Analysis in the flooding zone}

To determine the pressure amplification or attenuation effects of the pressure pulses along the pipeline, maximum pressure values at each transducer are compared. For each pulse, the maximum amplitudes registered by the pressure transducers located along the pipeline were recorded and their ratio with the maximum inlet pressure was determined. The maximum amplitude of a signal was defined by the two data points having the largest deviation from the moving average of the corresponding signal. One point corresponds to the largest deviation above the moving average (upper deviation) and the other to the largest deviation below the moving average (lower deviation). Once all the ratios were calculated for each pulse, the average pressure amplification/attenuation was determined. Figure 36 shows an example of the maximum deviation pressures for a single pulse on P1 and P2.

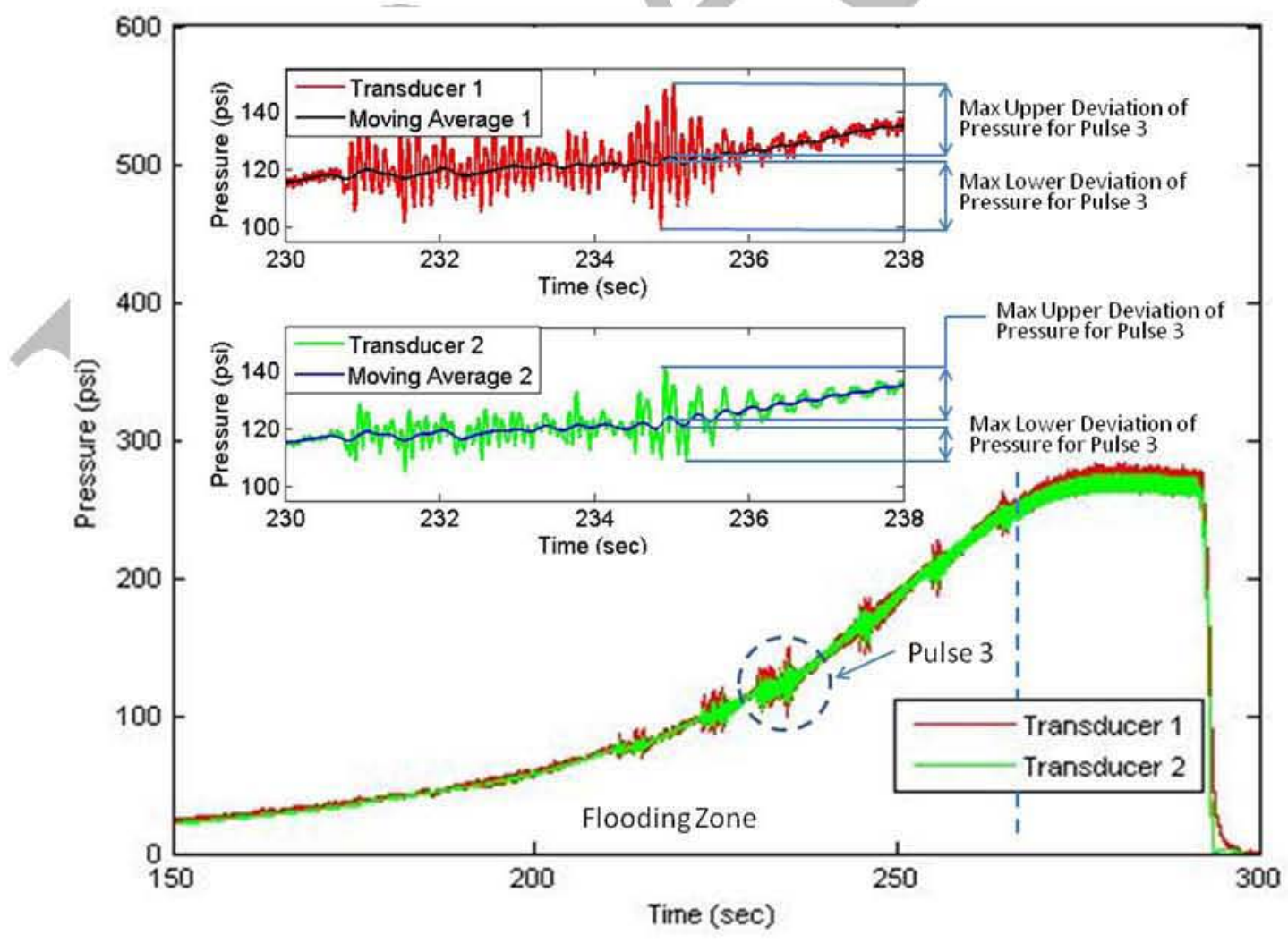

Figure 36. Pressure data analysis for $8 \mathrm{ft}$ Bentonite plug on the $310 \mathrm{ft}$ test bed. 
Table 10. Calculation of Upper and Lower Deviation from Moving Average

\begin{tabular}{|c|c|c|c|c|c|c|c|c|}
\hline 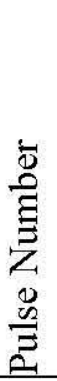 & 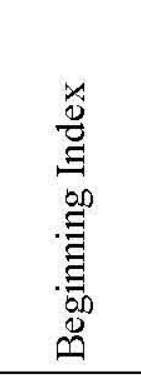 & 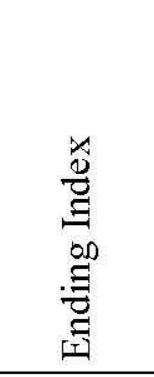 & 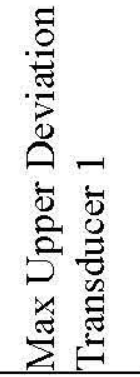 & 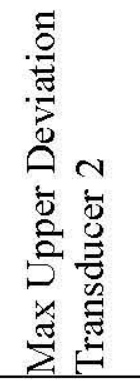 & 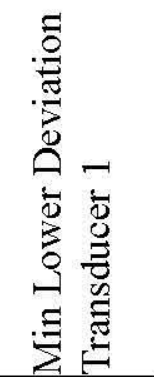 & 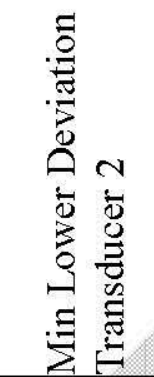 & 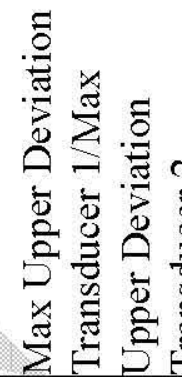 & 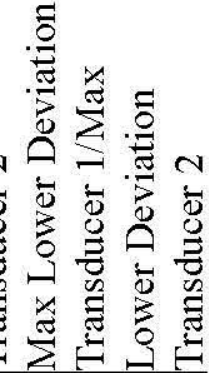 \\
\hline 1 & 211548 & 218276 & 10.252 & 6.543 & -8.698 & -4.270 & 0.638 & 0.491 \\
\hline 2 & 223155 & 229314 & 17.526 & 9.725 & -15.023 & -6.845 & 0.555 & 0.456 \\
\hline 3 & 230847 & 238522 & 25.402 & 17.723 & -23.325 & -12.048 & 0.698 & 0.517 \\
\hline 4 & 244715 & 249743 & 19.112 & 12.191 & -20.543 & -13.905 & 0.638 & 0.677 \\
\hline 5 & 254741 & 258579 & 19.829 & 9.933 & -20.043 & -12.740 & 0.501 & 0.636 \\
\hline 6 & 264578 & 267602 & 17.753 & 7.898 & -13.152 & -8.947 & 0.445 & 0.680 \\
\hline & & & & & & & 0.579 & 0.576 \\
\hline
\end{tabular}

Table 10 shows an example of the calculation of the maximum deviations from the moving average for 6 consecutive pulses in the unsteady region at P1 and P2. This data corresponds to testing trials for the $8 \mathrm{ft}$ Bentonite plugs on $310 \mathrm{ft}$ test bed.

\section{Analysis in the target pressure zone}

As shown in Figure 36, after the pipeline was filled, the average pressure levels off and the resulting disturbances from individual pressures pulses were difficult to discern with respect to the nominal pressure values. Therefore, a different approach was taken for data analysis in this region. The maximum pressure values and their corresponding time indexes were extracted for each signal. Similar to the approach used in the flooding zone, the maximum amplitude of a signal was defined by the two data points having the largest deviations from the moving average of the corresponding signal. Figure 37 shows an example of the how the maximum deviations were determined during the target pressure zone. One value corresponds to the largest deviation above the moving average and another to the largest deviation below the moving average. These two values provide a band within which all points for each of the signals should fall inside.

In order to provide a more detailed relationship between the pressure readings during unplugging, the ratios of the running moving averages were calculated at each time index. Maximum values for these ratios were then extracted for comparison. 


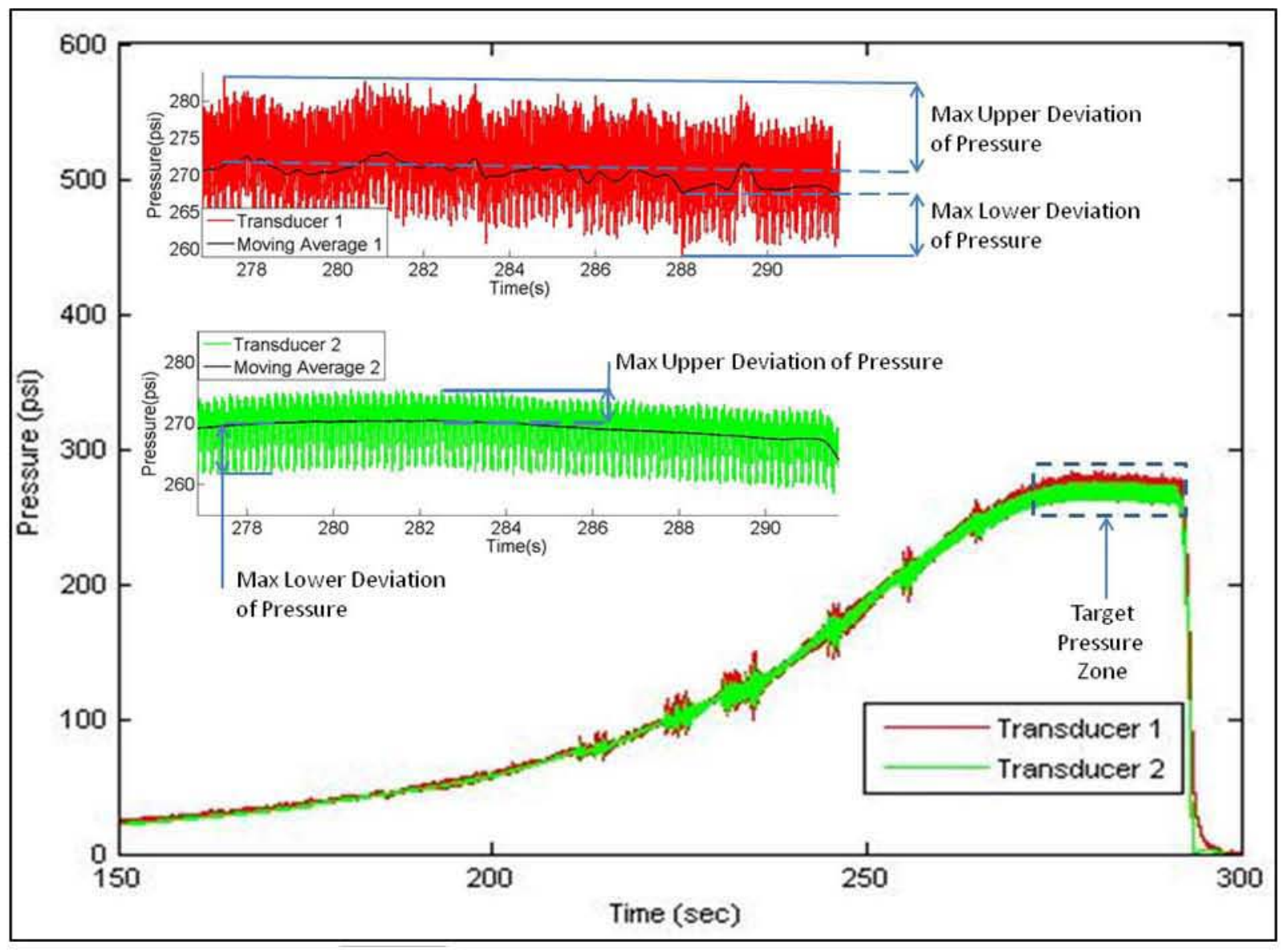

Figure 37. Pressure data for $8 \mathrm{ft}$ Bentonite plug using the $310 \mathrm{ft}$ test bed showing analysis.

As shown in Table 5 through Table 8 , parametric tests were conducted to determine how the control parameters used with the Hydrokinetics process affect the pipeline. In contrast to the pressure signature observed in the flooding zone for the unplugging experiments, the parameter testing data did not present well defined pressure disturbances (as with the target pressure zones). However, the vibration data showed areas of disturbances that appear at the same frequency as the known input frequency of the pulses generated from the AIMM's console. Based on the vibration areas of disturbances, the pressure data was divided into pulses as shown in Figure 38 .

For each pulse, the maximum amplitudes registered by the pressure transducers are compared by creating pressure ratios. The maximum amplitude of a signal is defined by the two data points having the largest deviation from the moving average of the corresponding signal. One value corresponds to the largest deviation above the moving average (upper deviation) and another to the largest deviation below the moving average (lower deviation). Once all the ratios are calculated, their average is computed. 


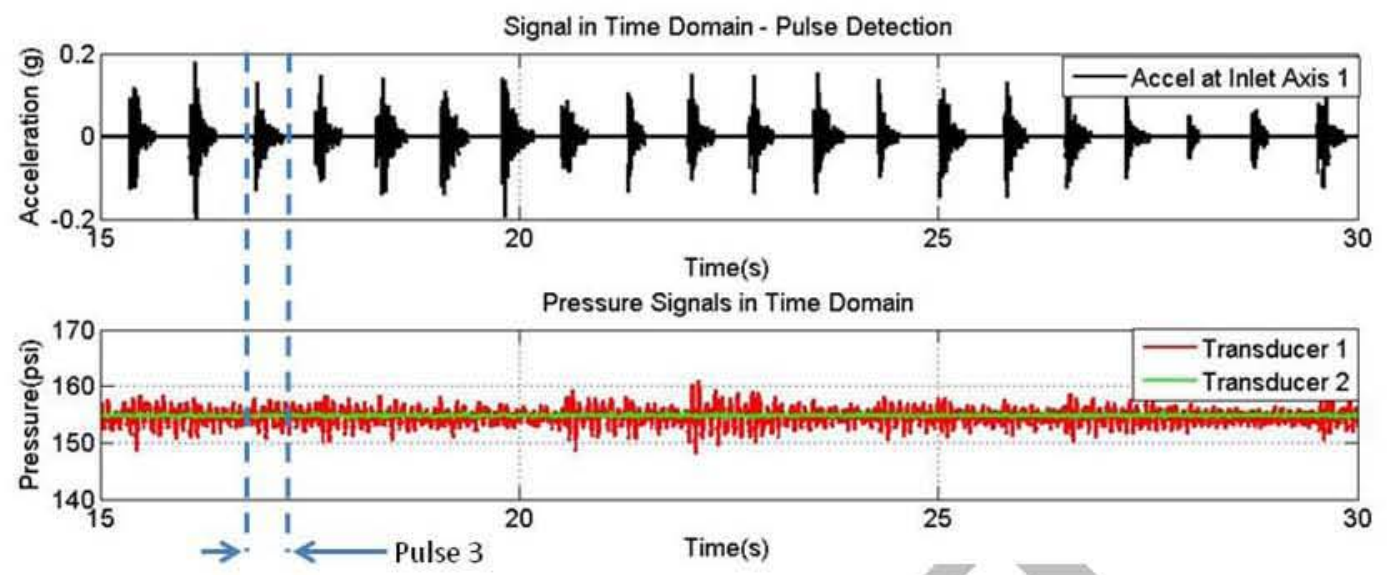

Detail View Pulse 3 Transducer 1

Detail View Pulse 3 Transducer 2
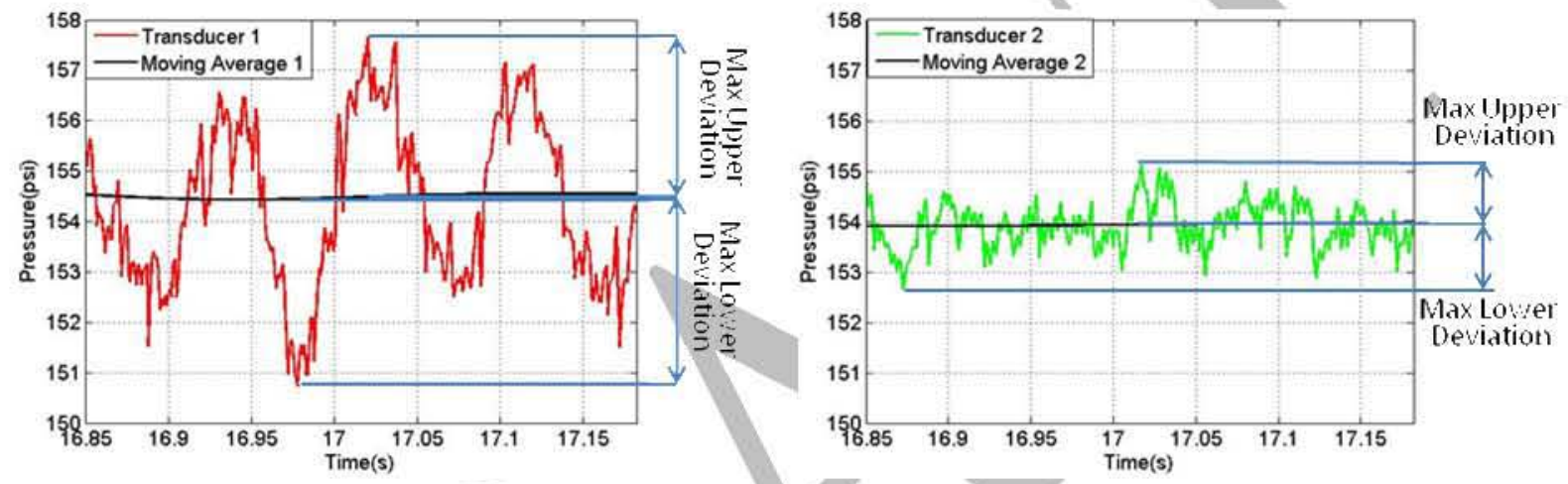

Figure 38. Parametric data for the $310 \mathrm{ft}$ test bed with a target pressure of $150 \mathrm{psi}$.

\section{Effect of Distance to the Plug on Pressure in Pipeline}

Understanding the attenuation/amplification of the propagation of pressure waves generated by the Hydrokinetic process is key to evaluating this technology. In this section, pressure ratios at three pipeline lengths are calculated and presented.

For the unplugging trials, two pressure ratios were obtained using two methods in the target pressure zone: 1) moving average pressures, 2) maximum deviation in pressure from the moving average. For the flooding zone, only the maximum deviation in pressure from the moving average was considered. Table 11 shows pressure ratios obtained for the moving average in the target pressure zone, where $\mathrm{P} 2 / \mathrm{P} 1, \mathrm{P} 4 / \mathrm{P} 3$ and $\mathrm{P} 6 / \mathrm{P} 1$ represent the effects of different lengths and number of elbows. For each ratio calculated, a mean value for the pressure ratios (shown within a range, eg: $0.987-1.003$ ) and the positive and negative deviations from the mean value (indicated by the + and - signs) are shown in Table 11.

The mean pressure ratios found to depart the farthest from the value of 1 were the maximum amplification or attenuation of the mean pressures along the pipeline. Results from the moving average pressure ratios indicated that the differences were generally not significant enough to detect trends for the pipe lengths. This was not the case for the maximum deviation in pressure calculations. 
Table 11. Effect of Pipeline Length on Moving Average Pressures during Target Pressure Zone

\begin{tabular}{|c|c|c|c|c|c|c|}
\hline & \multicolumn{6}{|c|}{ Bentonite $(8 \mathrm{ft})$} \\
\hline & \multicolumn{2}{|r|}{$\mathrm{P} 2 / \mathrm{P} 1$} & \multicolumn{2}{|c|}{$\mathrm{P} 4 / \mathrm{P} 3$} & \multicolumn{2}{|r|}{$\mathrm{P} 6 / \mathrm{P} 1$} \\
\hline $\begin{array}{c}310 \\
\mathrm{ft}\end{array}$ & $\begin{array}{c}0.987 \\
- \\
1.003 \\
\end{array}$ & $+0.449 /-0.934$ & $\begin{array}{c}0.999- \\
1.001\end{array}$ & $+0.394 /-0.229$ & $\begin{array}{c}0.993 \\
- \\
1.009\end{array}$ & $+0.078 /-0.096$ \\
\hline $\begin{array}{c}646 \\
\mathrm{ft}\end{array}$ & $\begin{array}{c}0.989 \\
- \\
1.001\end{array}$ & $+0.487 /-0.654$ & $\begin{array}{c}0.986- \\
1.011\end{array}$ & $+0.237 /-0.199$ & $\begin{array}{c}0.992 \\
- \\
1.009\end{array}$ & $+0.053 /-0.053$ \\
\hline $\begin{array}{c}1822 \\
\mathrm{ft}\end{array}$ & $\begin{array}{c}0.985 \\
- \\
1.022\end{array}$ & $+0.06 /-.062$ & $\begin{array}{c}0.958- \\
1.042\end{array}$ & $+0.424 /-0.458$ & $\begin{array}{c}0.965 \\
- \\
1.041\end{array}$ & $+0.015 /-0.015$ \\
\hline & \multicolumn{6}{|c|}{ Bentonite $(12 \mathrm{ft})$} \\
\hline & \multicolumn{2}{|r|}{$\mathrm{P} 2 / \mathrm{P} 1$} & \multicolumn{2}{|c|}{$\mathrm{P} 4 / \mathrm{P} 3$} & \multicolumn{2}{|r|}{$\mathrm{P} 6 / \mathrm{P} 1$} \\
\hline $\begin{array}{c}310 \\
\mathrm{ft}\end{array}$ & $\begin{array}{c}0.985 \\
- \\
1.000\end{array}$ & $+.617 /-.998$ & $\begin{array}{c}0.994- \\
1.001\end{array}$ & $+.240 /-.168$ & $\begin{array}{c}0.994 \\
- \\
1.008\end{array}$ & $+.102 /-.055$ \\
\hline $\begin{array}{c}646 \\
\mathrm{ft}\end{array}$ & $\begin{array}{c}0.994 \\
- \\
1.000 \\
\end{array}$ & $+.581 /-1.088$ & $\begin{array}{c}0.991- \\
1.000\end{array}$ & $+.177 /-.124$ & $\begin{array}{c}0.995 \\
- \\
1.005 \\
\end{array}$ & $+.031 /-.046$ \\
\hline \multirow[t]{3}{*}{$\begin{array}{c}1822 \\
\mathrm{ft}\end{array}$} & $\begin{array}{c}0.992 \\
- \\
1.003\end{array}$ & $+.112 /-.120$ & $\begin{array}{c}0.977= \\
1.026\end{array}$ & $+.595 /-.800$ & $\begin{array}{c}0.986 \\
- \\
1.016\end{array}$ & $+.048 /-.047$ \\
\hline & \multicolumn{6}{|c|}{$\operatorname{Kmag}(4 \mathrm{ft})$} \\
\hline & \multicolumn{2}{|r|}{$\mathrm{P} 2 / \mathrm{P} 1$} & \multicolumn{2}{|c|}{$\mathrm{P} 4 / \mathrm{P} 3$} & \multicolumn{2}{|r|}{$\mathrm{P} 6 / \mathrm{P} 1$} \\
\hline $\begin{array}{c}310 \\
\mathrm{ft}\end{array}$ & $\begin{array}{c}0.999 \\
- \\
1.001 \\
\end{array}$ & $+.376 /-.403$ & $\begin{array}{c}0.999- \\
1.005\end{array}$ & $+.283 /-.328$ & $\begin{array}{c}1.001 \\
- \\
1.009 \\
\end{array}$ & $+.072 /-.070$ \\
\hline $\begin{array}{c}646 \\
\mathrm{ft}\end{array}$ & $\begin{array}{r}0.998 \\
-.999\end{array}$ & $+.434 /-.351$ & $\begin{array}{c}0.999- \\
1.004\end{array}$ & $+.096 /-.110$ & $\begin{array}{c}0.999 \\
- \\
1.004\end{array}$ & $+.024 /-.017$ \\
\hline \multirow[t]{3}{*}{$\begin{array}{c}1822 \\
\mathrm{ft}\end{array}$} & $\begin{array}{c}0.983 \\
- \\
1.021\end{array}$ & $+.111 /-.125$ & $\begin{array}{c}0.960- \\
1.041\end{array}$ & $+.245 /-.248$ & $\begin{array}{c}1.023 \\
- \\
1.211\end{array}$ & $+.374 /-.489$ \\
\hline & \multicolumn{6}{|c|}{$\mathrm{Na}-\mathrm{Al}-\mathrm{Si}(4 \mathrm{ft})$} \\
\hline & \multicolumn{2}{|r|}{$\mathrm{P} 2 / \mathrm{P} 1$} & \multicolumn{2}{|c|}{$\mathrm{P} 4 / \mathrm{P} 3$} & \multicolumn{2}{|r|}{$\mathrm{P} 6 / \mathrm{P} 1$} \\
\hline $\begin{array}{c}310 \\
\mathrm{ft}\end{array}$ & $\begin{array}{c}0.995 \\
- \\
1.001\end{array}$ & $+.49 /-.388$ & $\begin{array}{c}1.000- \\
1.011\end{array}$ & $+.272 /-.393$ & $\begin{array}{c}0.999 \\
- \\
1.013\end{array}$ & $+.103 /-.088$ \\
\hline $\begin{array}{c}646 \\
\mathrm{ft}\end{array}$ & $\begin{array}{c}0.929 \\
- \\
1.012 \\
\end{array}$ & $+.22 /-.319$ & $\begin{array}{c}0.997- \\
1.014\end{array}$ & $+.309 /-.222$ & $\begin{array}{c}0.933 \\
- \\
1.024 \\
\end{array}$ & $+.039 /-.049$ \\
\hline $\begin{array}{c}1822 \\
\mathrm{ft}\end{array}$ & $\begin{array}{c}0.994 \\
- \\
1.026\end{array}$ & $+.138 /-.129$ & $\begin{array}{c}0.993- \\
1.059\end{array}$ & $+.306 /-.263$ & $\begin{array}{c}0.999 \\
- \\
1.077\end{array}$ & $+.019 /-.018$ \\
\hline
\end{tabular}


In Table $11, \mathrm{P} 6 / \mathrm{P} 1$ is the pressure ratio from inlet to outlet which demonstrated a significant attenuation in the pressure from the inlet to the exit. This is likely due to the remaining air in the pipeline after filling. For increasing pipe length it is also clear that the pressure ratio decreases for the $8 \mathrm{ft}$ Bentonite and Na-Al-Si plugs. For the other plugs, the $646 \mathrm{ft}$ pipe length has slightly unexpected results. This is likely attributed to the various amounts of air in the pipeline for each of the trials. It should be noted that the distance between P1 and P2 and between P3 and P4 are different for each of the pipe lengths.

In Figure 39 the change in the deviation in pressure with respect to the distance between sensors during the target zone is given for each test bed. It was observed that as the distance between the pressure transducers increase the fluctuations around the nominal pressure in the target zone shrink more except at the $1822 \mathrm{ft}$ case. In the $1822 \mathrm{ft}$ test bed, the deviation from the nominal pressure showed a change in the trend for the ratio P4/P3 at all of the unplugging trials. The plots for $310 \mathrm{ft}$ and $646 \mathrm{ft}$ pipelines showed that as the distance between sensors increased the final pressure observed close to the plug approached the mean average value, meaning that the fluctuations die out as the distance to the plug location is approached.

Table 12 shows the ratios of the maximum deviation in pressure from the mean during the flooding zone for the unplugging trials. The deviations presented here were calculated for each disturbance region that was captured during the flooding zone. The values shown in Table 12 are the final average values of the total number of ratios calculated for each flooding zone.

In Figure 40 the corresponding plots show that a similar trend in pressure ratio is observed for increasing distance in the pipeline. The fluctuations in the pressures reduced significantly compared to the inlet pressure. For almost every single pulsation created at the AIMM control box, the resulting disturbance in the pipeline pressure distribution reduced by $98.6 \%$ over the first straight pipe section of $42 \mathrm{ft}$ in length. This value goes up to $99.8 \%$ from the third transducer to the fourth which is $189 \mathrm{ft}$ apart from each other, connected with one $90^{\circ}$ elbow. There is still an ambiguous amplification of the pressure fluctuations in the $1822 \mathrm{ft}$ pipeline for cases with $8 \mathrm{ft}$ Bentonite and $4 \mathrm{ft}$ Kmag plugs.

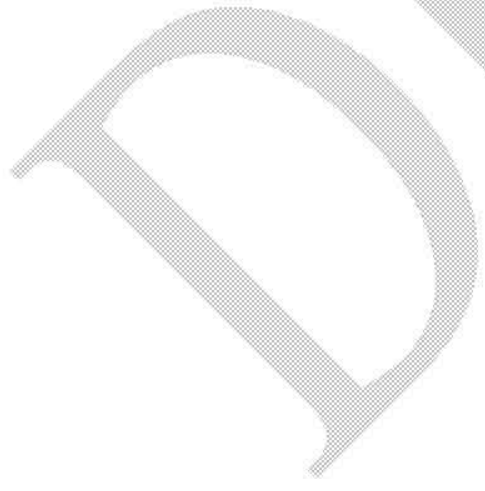


Table 12. Effect of Pipe Length on Maximum Deviation in Pressure from the Mean During Flooding Zone

\begin{tabular}{|c|c|c|c|c|c|c|}
\hline & \multicolumn{6}{|c|}{ Bentonite $(8 \mathrm{ft})$} \\
\hline & \multicolumn{2}{|c|}{$\mathrm{P} 2 / \mathrm{P} 1$} & \multicolumn{2}{|c|}{$\mathrm{P} 4 / \mathrm{P} 3$} & \multicolumn{2}{|c|}{$\mathrm{P} 6 / \mathrm{P} 1$} \\
\hline & Upper & Lower & Upper & Lower & Upper & Lower \\
\hline $310 \mathrm{ft}$ & 0.579 & 0.576 & 0.198 & 0.194 & 0.067 & 0.087 \\
\hline $646 \mathrm{ft}$ & 0.291 & 0.429 & 0.711 & 0.700 & 0.119 & 0.193 \\
\hline \multirow[t]{4}{*}{$1822 \mathrm{ft}$} & 0.073 & 0.069 & 0.765 & 0.759 & 0.037 & 0.039 \\
\hline & \multicolumn{6}{|c|}{ Bentonite $(12 \mathrm{ft})$} \\
\hline & \multicolumn{2}{|c|}{$\mathrm{P} 2 / \mathrm{P} 1$} & \multicolumn{2}{|c|}{$\mathrm{P} 4 / \mathrm{P} 3$} & \multicolumn{2}{|c|}{$\mathrm{P} 6 / \mathrm{P} 1$} \\
\hline & Upper & Lower & Upper & Lower & Upper & Lower \\
\hline $310 \mathrm{ft}$ & 0.409 & 0.494 & 0.312 & 0.339 & 0.065 & 0.086 \\
\hline $646 \mathrm{ft}$ & 0.403 & 0.520 & 0.674 & 0.685 & 0.117 & 0.176 \\
\hline \multirow[t]{4}{*}{$1822 \mathrm{ft}$} & 0.196 & 0.183 & 0.921 & 0.863 & 0.094 & 0.095 \\
\hline & \multicolumn{6}{|c|}{$\operatorname{Kmag}(4 \mathrm{ft})$} \\
\hline & \multicolumn{2}{|c|}{$\mathrm{P} 2 / \mathrm{P} 1$} & \multicolumn{2}{|c|}{$\mathrm{P} 4 / \mathrm{P} 3$} & \multicolumn{2}{|c|}{$\mathrm{P} 6 / \mathrm{P} 1$} \\
\hline & Upper & Lower & Upper & Lower & Upper & Lower \\
\hline $310 \mathrm{ft}$ & N/A & N/A & $\mathrm{N} / \mathrm{A}$ & $\mathrm{N} / \mathrm{A}$ & N/A & N/A \\
\hline $646 \mathrm{ft}$ & 0.257 & 0.267 & 0.676 & 0.619 & 0.116 & 0.123 \\
\hline \multirow[t]{2}{*}{$1822 \mathrm{ft}$} & 0.054 & 0.064 & 0.505 & 0.469 & 0.016 & 0.019 \\
\hline & \multicolumn{6}{|c|}{$\mathrm{Na}-\mathrm{Al}-\mathrm{Si}(4 \mathrm{ft})$} \\
\hline 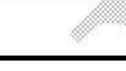 & \multicolumn{2}{|c|}{$\mathrm{P} 2 / \mathrm{P} 1$} & \multicolumn{2}{|c|}{$\mathrm{P} 4 / \mathrm{P} 3$} & \multicolumn{2}{|c|}{$\mathrm{P} 6 / \mathrm{P} 1$} \\
\hline & Upper & Lower & Upper & Lower & Upper & Lower \\
\hline $310 \mathrm{ft}$ & $\mathrm{N} / \mathrm{A}$ & $\mathrm{N} / \mathrm{A}$ & N/A & N/A & N/A & N/A \\
\hline $646 \mathrm{ft}$ & 0.345 & 0.382 & 0.429 & 0.406 & 0.060 & 0.054 \\
\hline $1822 \mathrm{ft}$ & 0.193 & 0.164 & 0.770 & 0.785 & 0.092 & 0.094 \\
\hline
\end{tabular}



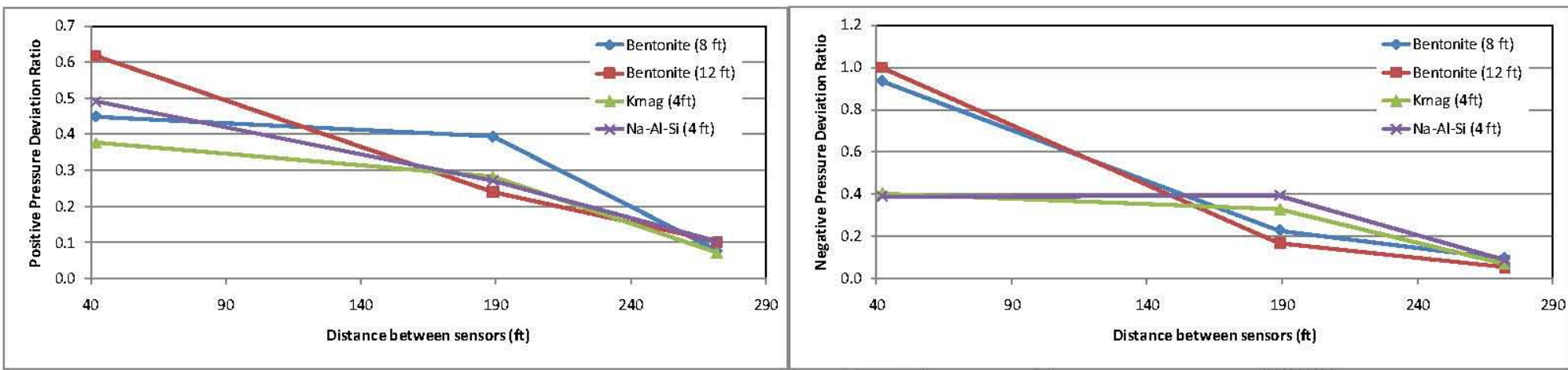

$(310 \mathrm{ft})$
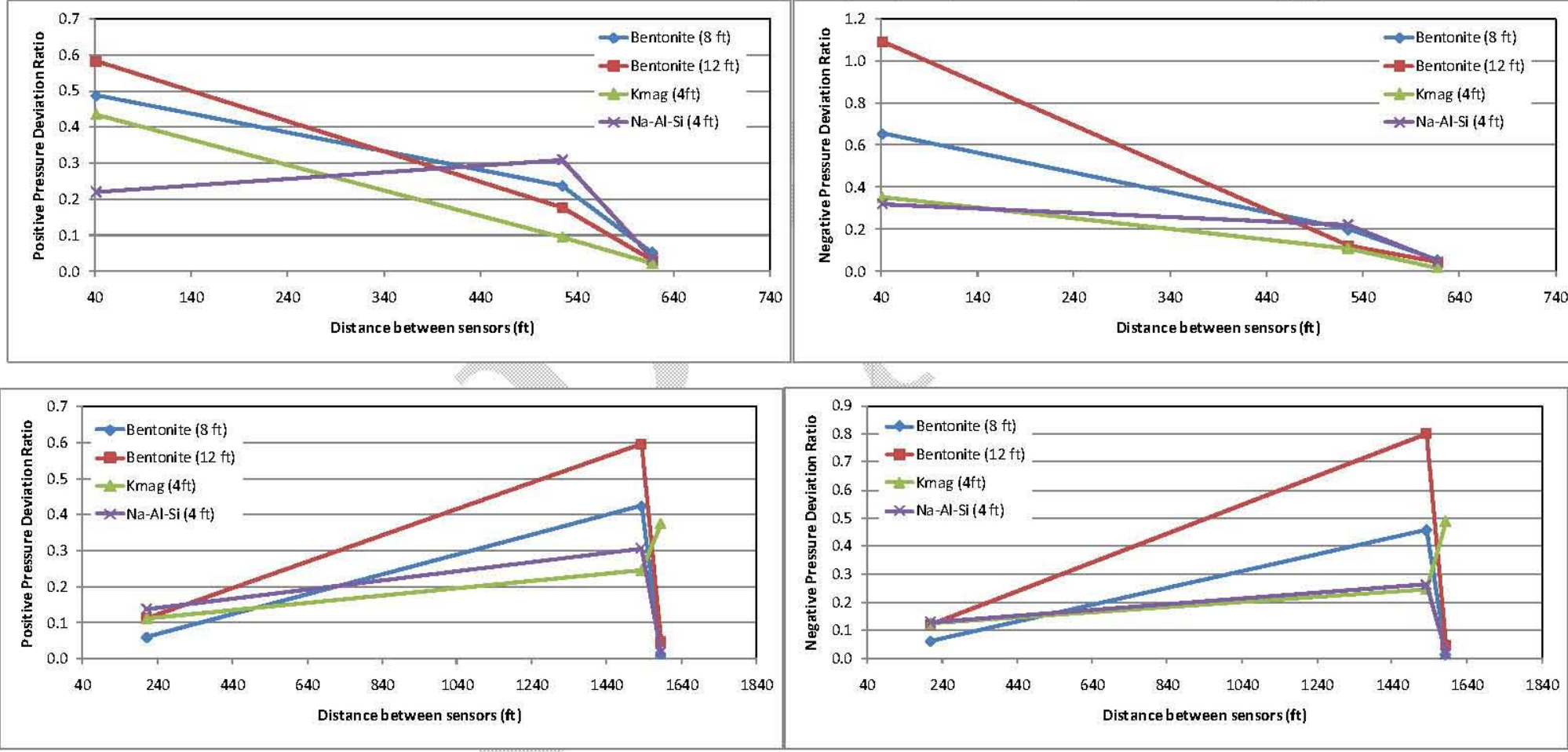

Figure 39. Pressure deviation with respect to distance between sensors at three different pipelines during the target zone. 

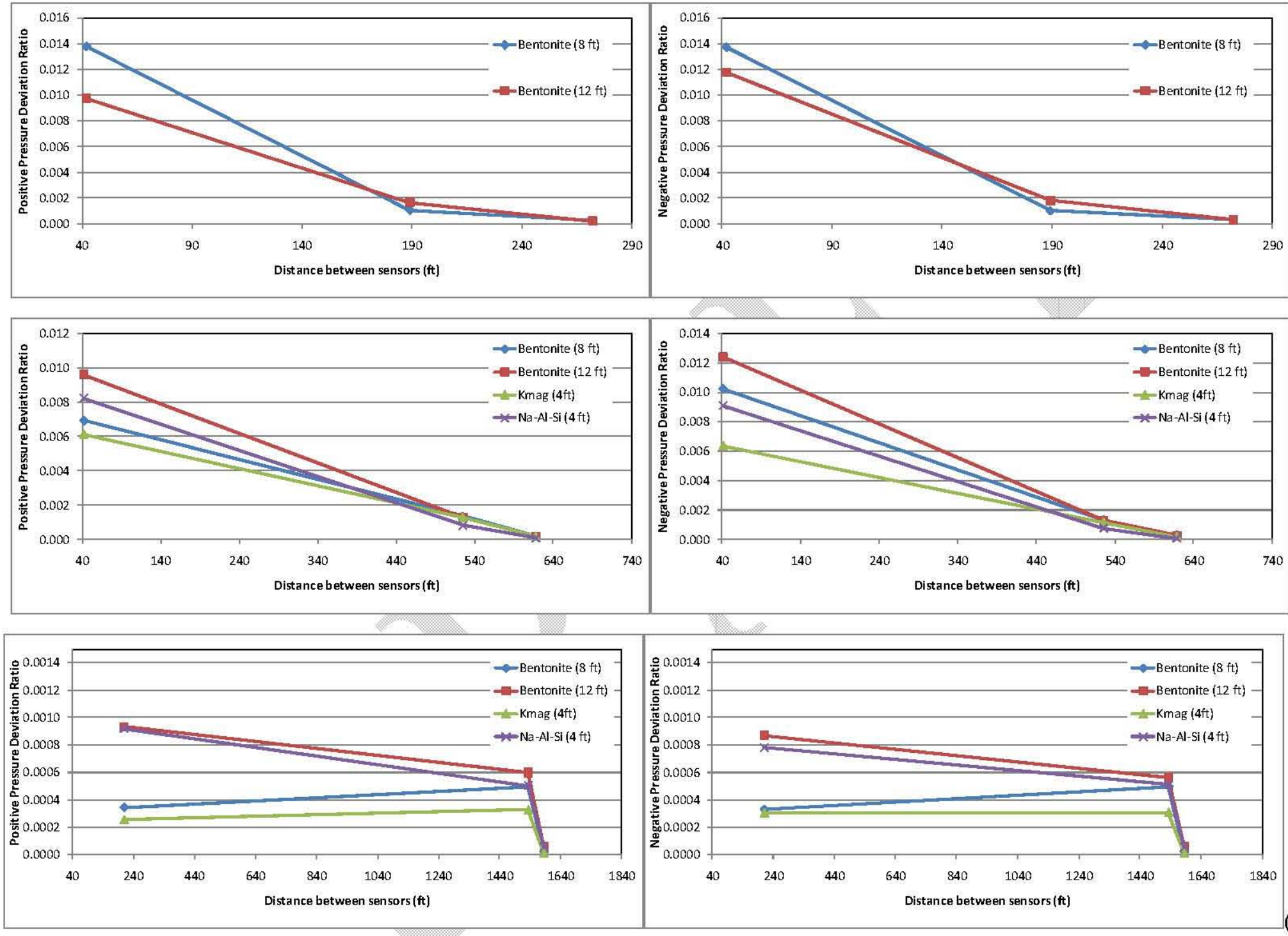

Figure 40. Pressure deviation with respect to distance between sensors at three different pipelines during the flooding zone. 


\section{Effects of Elbows on Pressure Propagation}

The waste transfer pipelines usually have numerous elbows and sweeps. Any kind of turn in the pipeline can create a challenge for any unplugging technology to perform effectively over long distances. The capability to operate in a pipeline with many elbows was found to be one of the primary criteria that can qualify an unplugging technology for deployment at a DOE site.

To evaluate the effect of elbows on the pressure propagation, the pressure ratio $\mathrm{P} 3 / \mathrm{P} 2$ was obtained using two methods in the target pressure zone: 1) moving average pressures, 2) maximum deviation in pressure from the moving average. For the flooding zone, only the maximum deviation in pressure from the moving average was utilized. The ratios found to depart the farthest and closest from 1 are the maximum amplification or attenuation of the pressure across an elbow. Table 13 through Table 15 show the results for P3/P2 for the unplugging trials.

Table 13. Effect of Elbows on Mean Pressures during Target Pressure Zone

\begin{tabular}{|c|c|c|c|c|c|c|c|c|}
\hline & \multicolumn{2}{|c|}{ Bentonite $(8 \mathrm{ft})$} & \multicolumn{2}{c|}{ Bentonite $(12 \mathrm{ft})$} & \multicolumn{2}{c|}{ Kmag (4ft) } & \multicolumn{2}{c|}{ Na-Al-Si (4 ft) } \\
\hline & \multicolumn{2}{|c|}{ Range } & \multicolumn{2}{c|}{ Range } & \multicolumn{2}{c|}{ Range } & \multicolumn{2}{c|}{ Range } \\
\hline $310 \mathrm{ft}$ & 0.997 & 1.001 & 0.995 & 0.998 & 0.999 & 1.000 & 0.998 & 1.001 \\
\hline $646 \mathrm{ft}$ & 0.999 & 1.001 & 0.998 & 0.999 & 1.000 & 1.002 & 0.997 & 1.001 \\
\hline $1822 \mathrm{ft}$ & 0.996 & 1.003 & 0.996 & 0.999 & 0.995 & 1.003 & 0.994 & 1.002 \\
\hline
\end{tabular}

Table 14. Effect of Elbows on Pressure Deviations from the Mean during Target Pressure Zone

\begin{tabular}{|c|c|c|c|c|c|c|c|c|}
\hline & \multicolumn{2}{|c|}{ Bentonite $(8 \mathrm{ft})$} & \multicolumn{2}{c|}{ Bentonite $(12 \mathrm{ft})$} & \multicolumn{2}{c|}{ Kmag $(4 \mathrm{ft})$} & \multicolumn{2}{c|}{ Na-Al-Si $(4 \mathrm{ft})$} \\
\hline & Upper & Lower & Upper & Lower & Upper & Lower & Upper & Lower \\
\hline $310 \mathrm{ft}$ & 1.082 & 1.151 & 1.109 & 1.079 & 0.983 & 0.917 & 0.985 & 1.038 \\
\hline $646 \mathrm{ft}$ & 1.151 & 0.988 & 1.110 & 1.158 & 1.117 & 1.090 & 1.188 & 1.157 \\
\hline $1822 \mathrm{ft}$ & 1.350 & 1.231 & 1.431 & 1.270 & 1.290 & 1.208 & 1.183 & 1.464 \\
\hline
\end{tabular}

Table 15. Effect of Elbows on Pressure Deviations from the Mean during Flooding

\begin{tabular}{|c|c|c|c|c|c|c|c|c|}
\hline & \multicolumn{2}{|c|}{ Bentonite $(8 \mathrm{ft})$} & \multicolumn{2}{c|}{ Bentonite $(12 \mathrm{ft})$} & \multicolumn{2}{c|}{ Kmag $(4 \mathrm{ft})$} & \multicolumn{2}{c|}{ Na-Al-Si (4 ft) } \\
\hline & Upper & Lower & Upper & Lower & Upper & Lower & Upper & Lower \\
\hline $310 \mathrm{ft}$ & 1.326 & 1.307 & 1.434 & 1.300 & N/A & N/A & N/A & N/A \\
\hline $646 \mathrm{ft}$ & 1.138 & 1.113 & 1.324 & 1.388 & 1.226 & 1.318 & 1.277 & 1.150 \\
\hline $1822 \mathrm{ft}$ & 1.314 & 1.386 & 1.110 & 1.206 & 1.373 & 1.355 & 1.212 & 1.229 \\
\hline
\end{tabular}

For the calculations in the target pressure zone, Table 14 shows that generally the pressure fluctuations are amplified after the elbow when compared to the fluctuations in pressure before the elbow. Since the mean average values of the pressures are very close to 1 , it would be sufficient to consider the ratios of the deviations from the mean in order to obtain conclusions from this analysis. 
It should be mentioned that the pressure ratios presented in Table 13 through Table 15 only represent the effect of the elbow and not the pipeline length since the sensors were placed right on the inlet and exit of the elbow. Another important point to mention is that the elbows used in the current work were not perfect $90^{\circ}$ sweeping elbows, rather three way ball valves with one end closed creating a $\mathrm{T}$ shape with two orthogonal ends open. The amplifications seen in the fluctuations after the elbow can be a result of the pressure waves hitting the closed end of the ball valve and being reflected into the exit of the elbow.

Table 14 shows that generally the amplification increases with increasing pipeline length during the target pressure zone. However this does not hold for the flooding zone.

\section{Effect of Expansion Joint on Pressure Propagation}

Expansion joints can also be present in transfer lines where they create a major deviation in the direction of flow in the pipeline (Figure 12). The expansion joint used in this study was made up of three 10-ft pipe sections and two $90^{\circ}$ sweeping elbows and two three-way ball valves. The expansion joint study was conducted during a parametric test where the pipeline was capped at the end, replacing the blockage. For cases where the expansion joint was not installed, a straight pipe section was used to by-pass the expansion joint by adjusting the three-way ball valve.

Using the maximum and minimum ratio of P5 to $\mathrm{P} 4$ that were placed right before and after the expansion joint, the moving average at each pulse and the average of the ratios for the disturbances were calculated.

Table 16. Effect of Expansion Joint (EJ) on Mean Pressures at $1822 \mathrm{ft}$ Test Bed

\begin{tabular}{|c|c|c|c|c|}
\hline & \multicolumn{4}{|c|}{$150 \mathrm{PSI}$} \\
\hline & \multicolumn{2}{|c|}{ EJ ON } & \multicolumn{2}{|c|}{ EJ OFF } \\
\hline RPM & \multicolumn{2}{|c|}{ Range } & \multicolumn{2}{|c|}{ Range } \\
\hline 1350 & 0.995 & 0.995 & 0.996 & 0.996 \\
\hline$\sim 1413$ & 0.995 & 0.996 & 0.995 & 0.996 \\
\hline 1450 & 0.995 & 0.995 & 0.995 & 0.995 \\
\hline & \multicolumn{4}{|c|}{200 PSI } \\
\hline & \multicolumn{2}{|c|}{$\mathrm{EJ} \mathrm{ON}$} & \multicolumn{2}{|c|}{ EJ OFF } \\
\hline RPM & \multicolumn{2}{|c|}{ Range } & \multicolumn{2}{|c|}{ Range } \\
\hline 1350 & 0.999 & 0.999 & 0.999 & 1.000 \\
\hline$\sim 1413$ & 0.999 & 1.000 & 1.000 & 1.000 \\
\hline 1450 & 0.999 & 1.000 & 0.999 & 0.999 \\
\hline & \multicolumn{4}{|c|}{250 PSI } \\
\hline & \multicolumn{2}{|c|}{ EJ ON } & \multicolumn{2}{|c|}{ EJ OFF } \\
\hline RPM & \multicolumn{2}{|c|}{ Range } & \multicolumn{2}{|c|}{ Range } \\
\hline 1350 & 1.002 & 1.002 & 1.000 & 1.000 \\
\hline$\sim 1413$ & 1.000 & 1.001 & 1.000 & 1.000 \\
\hline 1450 & 1.001 & 1.001 & 1.000 & 1.001 \\
\hline
\end{tabular}


Table 16 summarizes the findings from the parametric test with the expansion joint connected and disconnected from the pipeline. Although the values in Table 16 were found to be very close to 1 and therefore difficult to assess trends, it should be noted that as the inlet pressure is increased the mean pressures after the expansion joint increase slightly.

Using the maximum values of the positive and negative deviations in P5/P4 at each pulse, the average of the ratios for the disturbances was also calculated as shown below (Table 17).

As expected the pressure ratios (P5/P4) with the expansion joint removed were greater than with the expansion joint connected. Especially for lower pump RPMs, the expansion joint reduced the fluctuations around the mean pressure at the plug location, which was observed for all three different inlet pressures. However an increase in the RPM value did not result in a particular trend in the effect of expansion joint.

Table 17. Effect of Expansion Joint (EJ) on Pressure Deviations from the Mean at $1822 \mathrm{ft}$ Test Bed

\begin{tabular}{|c|c|c|c|c|c|}
\hline & \multicolumn{5}{|c|}{150 PSI } \\
\hline & \multicolumn{2}{|c|}{ EJ ON } & \multicolumn{2}{c|}{ EJ OFF } \\
\hline RPM & Upper & Lower & Upper & Lower \\
\hline 1350 & 0.469 & 0.457 & 0.548 & 0.540 \\
\hline$\sim 1413$ & 0.510 & 0.490 & 0.530 & 0.519 \\
\hline 1450 & 0.501 & 0.488 & 0.547 & 0.535 \\
\hline & \multicolumn{5}{|c|}{200 PSI } \\
\hline & \multicolumn{2}{|c|}{ EJ ON } & EJ OFF \\
\hline RPM & Upper & Lower & Upper & Lower \\
\hline 1350 & 0.459 & 0.461 & 0.487 & 0.488 \\
\hline 1413 & 0.450 & 0.477 & 0.501 & 0.487 \\
\hline 1450 & 0.484 & 0.466 & 0.488 & 0.487 \\
\hline & & 250 PSI \\
\hline & & EJ ON & EJ OFF \\
\hline RPM & Upper & Lower & Upper & Lower \\
\hline 1350 & 0.456 & 0.455 & 0.515 & 0.480 \\
\hline$\sim 1413$ & 0.437 & 0.450 & 0.489 & 0.476 \\
\hline 1450 & 0.424 & 0.421 & 0.491 & 0.496 \\
\hline
\end{tabular}

\section{Effect of Reducer on Pressure Propagation}

A similar analysis as compared to the above was conducted for the effect of reducers or area changes in the pipeline. The analysis with the reducer was conducted during a parametric trial at $310 \mathrm{ft}$ pipeline at $200 \mathrm{psi}$. Using the maximum and minimum ratios between the moving averages of P4 and P3 at each pulse, the average of the ratios for the disturbances was calculated (Table 18). 
Table 18. Effect of Reducer on Mean Pressure at $310 \mathrm{ft}$ Test Bed at $200 \mathrm{psi}$

\begin{tabular}{|c|c|c|c|c|}
\hline & \multicolumn{3}{|c|}{200 PSI } \\
\hline & \multicolumn{2}{|c|}{ Reducer ON } & \multicolumn{2}{c|}{ Reducer OFF } \\
\hline RPM & \multicolumn{2}{|c|}{ Range } & \multicolumn{2}{c|}{ Range } \\
\hline 1350 & 1.000 & 1.001 & 0.996 & 0.997 \\
\hline$\sim 1413$ & 0.997 & 0.999 & 0.996 & 0.998 \\
\hline 1450 & 0.996 & 0.999 & 0.996 & 0.998 \\
\hline
\end{tabular}

Using the maximum deviation from the mean $\mathrm{P} 4$ and $\mathrm{P} 3$ for each pulsation, the ratio of $\mathrm{P} 4 / \mathrm{P} 3$ was calculated and the final average value for all of the disturbances was shown below (Table 19). It can be observed that when the reducer was placed on the pipeline, the mean pressure slightly increased after the reducer while the fluctuations around those mean pressures were found to be generally amplified as well. The increase in pump frequency adversely affects the amplification of the pressure fluctuations and the mean pressures around the reducer.

Table 19. Effect of Reducer on Pressure Deviations from the Mean at $310 \mathrm{ft}$ Test Bed at 200 psi

\begin{tabular}{|c|c|c|c|c|}
\hline & \multicolumn{3}{|c|}{200 PSI } \\
\hline & \multicolumn{2}{|c|}{ Reducer ON } & \multicolumn{2}{c|}{ Reducer OFF } \\
\hline RPM & Upper & Lower & Upper & Lower \\
\hline 1350 & 0.353 & 0.338 & 0.365 & 0.344 \\
\hline$\sim 1413$ & 0.312 & 0.292 & 0.278 & 0.285 \\
\hline 1450 & 0.252 & 0.237 & 0.230 & 0.229 \\
\hline
\end{tabular}

\section{EFFECT OF PIPELINE LENGTH AND GEOMETRY ON VIBRATION}

In this section, the pipe wall acceleration data in two axes is used to investigate the effects of pipeline length and geometry on changes of pipe wall vibration characteristics from the inlet point to the plug section. The axes of acceleration measured during the unplugging process are shown in Figure 41. In the current analysis only Axis 1 and Axis 2 are reported since the vibration along Axis 3 had virtually no response. This is likely due to the boundary conditions imposed by the pipe clamps. The propagation of vibration was measured by comparing the maximum magnitude of the disturbance produced by AIMM Technologies at the inlet and the plug section. 


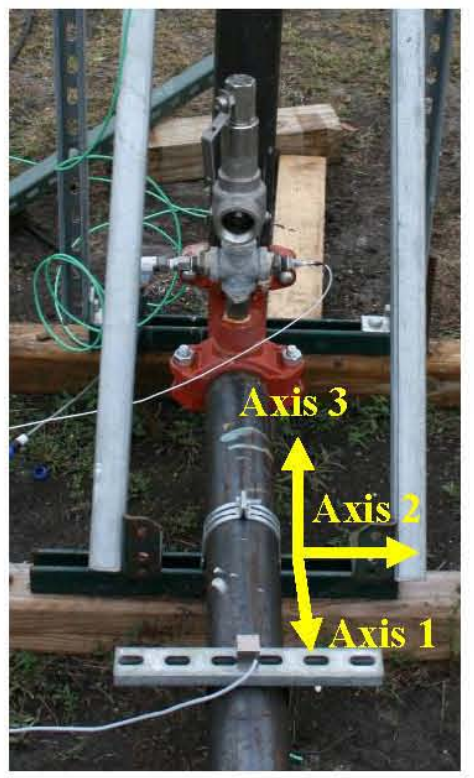

(a) Inlet section

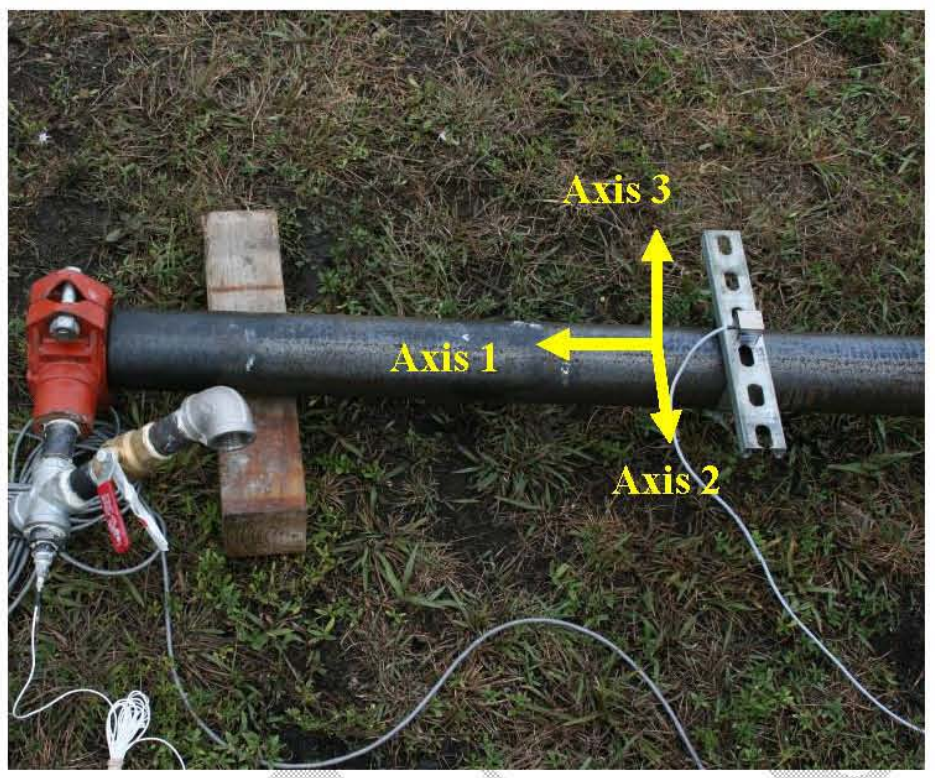

(b) Plug section

Figure 41. Orientation of accelerometers at the inlet section and plug sections.

In contrast to the pressure data, the vibration data does demonstrate zones associated with flooding the pipeline and attaining the target pressure. Therefore, the time indices for the pulses during the flooding zone, for the vibration analysis, are the same as those found in the pressure analysis. Additionally, the vibration analysis for the target pressure zone uses the same principles for finding the maximum deviations as found in the pressure analysis. The ratios for the mean averages are not included in the vibration analysis because both accelerometers provided readings that yield moving averages very close to zero.

The vibration analysis for the parametric data was again divided into pulses, and the maximum amplitude registered by the accelerometers (located at inlet and located at blockage) is compared by calculating their ratios as shown in Figure 42 . The maximum amplitude of a signal is defined by the two data points having the largest deviation from the moving average of the corresponding signal. One point corresponds to the largest deviation above the moving average (upper deviation) and the other to the largest deviation below the moving average (lower deviation). After the vibration ratios are calculated for all the pulses, both upper deviation and lower deviation averages are determined. 

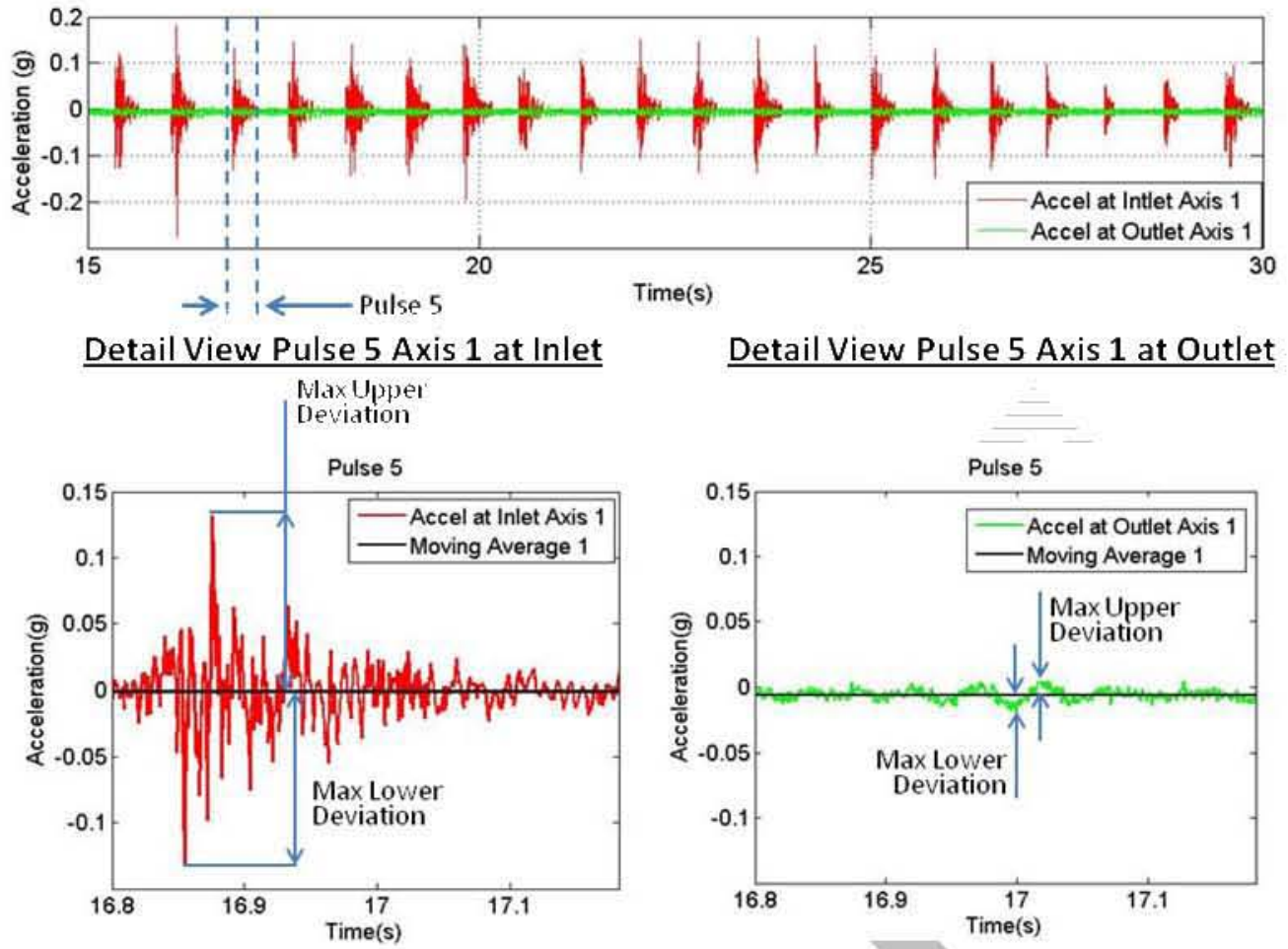

Detail View Pulse 5 Axis 1 at Outlet

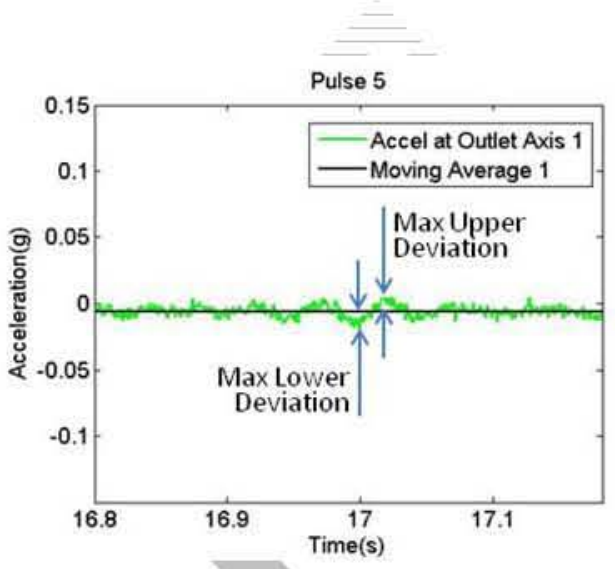

Figure 42. Parametric vibration data using the $310 \mathrm{ft}$ test bed with a target pressure of $150 \mathrm{psi}$.

\section{Effect of Distance to the Plug on Pipe Vibration}

In order to analyze the effects of distance to the plug on the translation of vibration through the pipeline, a region representing the general trend of the vibration characteristics was selected for each unplugging trial.

The maximum positive and negative deviation from the mean vibration was calculated within the region of interest at the inlet and the plug location. Since the mean values of vibration were zero only deviations were used in the analysis as shown in Table 20 for the target zone and Table 21 for the flooding zone.

Significant degradation in vibration at the plug location was observed for all of the unplugging cases, particularly at the longer pipe lengths. The reduction in vibration characteristics of the pipes at the plug location was observed to be between $61 \%$ and $97.9 \%$.

Table 20. Effect of Pipe Length on Vibration during Target Pressure Zone

\begin{tabular}{|c|c|c|c|c|c|c|c|c|}
\hline & \multicolumn{4}{|c|}{ Bentonite $(8 \mathrm{ft})$} & \multicolumn{4}{c|}{ Bentonite (12 ft) } \\
\hline & Accel 4/Accel 1 & Accel 5/Accel 2 & Accel 4/Accel 1 & Accel 5/Accel 2 \\
\hline & Upper & Lower & Upper & Lower & Upper & Lower & Upper & Lower \\
\hline $310 \mathrm{ft}$ & 0.240 & 0.235 & 0.169 & 0.188 & 0.361 & 0.393 & 0.262 & 0.160 \\
\hline $646 \mathrm{ft}$ & 0.056 & 0.090 & 0.072 & 0.058 & 0.081 & 0.075 & 0.112 & 0.092 \\
\hline $1822 \mathrm{ft}$ & 0.028 & 0.030 & 0.028 & 0.042 & 0.064 & 0.069 & 0.045 & 0.035 \\
\hline
\end{tabular}




\begin{tabular}{|c|c|c|c|c|c|c|c|c|}
\hline & \multicolumn{4}{|c|}{ Kmag (4ft) } & \multicolumn{4}{c|}{ Na-Al-Si (4ft) } \\
\hline & Accel 4/Accel 1 & \multicolumn{2}{|c|}{ Accel 5/Accel 2 } & Accel 4/Accel 1 & Accel 5/Accel 2 \\
\hline & Upper & Lower & Upper & Lower & Upper & Lower & Upper & Lower \\
\hline $310 \mathrm{ft}$ & 0.160 & 0.184 & N/A & N/A & 0.157 & 0.101 & 0.049 & 0.067 \\
\hline $646 \mathrm{ft}$ & 0.045 & 0.054 & 0.055 & 0.044 & 0.099 & 0.098 & 0.049 & 0.053 \\
\hline $1822 \mathrm{ft}$ & 0.022 & 0.021 & 0.037 & 0.037 & 0.036 & 0.023 & 0.052 & 0.053 \\
\hline
\end{tabular}

For the target pressure zone, as the distance to the plug location was increased for the same plug type, the vibration observed at the plug was reduced. However during the flooding zone, a similar trend was not discernible. In the flooding zone, the reduction in vibration at the plug section was found to be between $62.4 \%$ and $97.7 \%$.

Table 21. Effect of Pipe Length on Vibration during Flooding Zone

\begin{tabular}{|c|c|c|c|c|c|c|c|c|}
\hline & \multicolumn{4}{|c|}{ Bentonite $(8 \mathrm{ft})$} & \multicolumn{4}{c|}{ Bentonite $(12 \mathrm{ft})$} \\
\hline & Accel 4/Accel 1 & Accel 5/Accel 2 & \multicolumn{2}{|c|}{ Accel 4/Accel 1 } & Accel 5/Accel 2 \\
\hline & Upper & Lower & Upper & Lower & Upper & Lower & Upper & Lower \\
\hline $310 \mathrm{ft}$ & 0.087 & 0.091 & 0.198 & 0.126 & 0.218 & 0.131 & 0.346 & 0.376 \\
\hline $646 \mathrm{ft}$ & 0.091 & 0.076 & 0.091 & 0.093 & 0.171 & 0.130 & 0.103 & 0.121 \\
\hline $1822 \mathrm{ft}$ & 0.057 & 0.076 & 0.052 & 0.065 & 0.162 & 0.120 & 0.123 & 0.090 \\
\hline
\end{tabular}

\begin{tabular}{|c|c|c|c|c|c|c|c|c|}
\hline & \multicolumn{4}{|c|}{ Kmag (4ft) } & \multicolumn{4}{c|}{ Na-Al-Si (4ft) } \\
\hline & \multicolumn{2}{|c|}{ Accel 4/Accel 1 } & Accel 5/Accel 2 & \multicolumn{2}{c|}{ Accel 4/Accel 1 } & Accel 5/Accel 2 \\
\hline & Upper & Lower & Upper & Lower & Upper & Lower & Upper & Lower \\
\hline $310 \mathrm{ft}$ & 0.226 & 0.190 & N/A & N/A & 0.161 & 0.160 & 0.137 & 0.156 \\
\hline $646 \mathrm{ft}$ & 0.071 & 0.068 & 0.109 & 0.091 & 0.154 & 0.126 & 0.102 & 0.100 \\
\hline $1822 \mathrm{ft}$ & 0.023 & 0.025 & 0.122 & 0.062 & 0.042 & 0.036 & 0.047 & 0.055 \\
\hline
\end{tabular}

\section{Effect of Expansion Joint on Pipe Vibration}

In this section, the effects of the expansion joint are evaluated by utilizing parametric testing data. Maximum vibration data at the inlet and blockage are obtained, as before with upper and lower deviations accelerations ratios determined. Table 22 shows the vibration ratios for Axis 1 with the expansion joint on and off for various operating pressures and plunger pump speeds. It should be noted that the vibration ratios were small, which - means that very little vibration was transmitted to the blockage area. As expected, the trials with the expansion joint off have better attenuation rates than the trials with the expansion joint on. There did not appear to be a significant trend varying the pump RPM or the operating pressure. The vibration ratios for Axis 2 are shown in Table 23. For the 150 psi trials, the cases with the expansion joint off had slightly higher vibration ratios than the trials with the expansion joint on. This was not the case, however, for the other operating pressures. This change was possibly due to the nature of the imposed boundary conditions of the pipe clamps. 
Table 22. Effect of Expansion Joint (EJ) on Vibration - $1822 \mathrm{ft}$ Test bed, Axis 1 (Outlet/lnlet)

\begin{tabular}{|c|c|c|c|c|}
\hline & \multicolumn{4}{|c|}{ Accel 4/Accel 1} \\
\hline & \multicolumn{4}{|c|}{150 PSI } \\
\hline & \multicolumn{2}{|c|}{ EJ ON } & \multicolumn{2}{|c|}{ EJ OFF } \\
\hline RPM & Upper & Lower & Upper & Lower \\
\hline 1350 & 0.0175 & 0.0162 & 0.0260 & 0.0277 \\
\hline$\sim 1413$ & 0.0172 & 0.0158 & 0.0236 & 0.0252 \\
\hline 1450 & 0.0171 & 0.0156 & 0.0248 & 0.0254 \\
\hline & \multicolumn{4}{|c|}{ Accel 4/Accel 1} \\
\hline & \multicolumn{4}{|c|}{$200 \mathrm{PSI}$} \\
\hline & \multicolumn{2}{|c|}{ EJ ON } & \multicolumn{2}{|c|}{ EJ OFF } \\
\hline RPM & Upper & Lower & Upper & Lower \\
\hline 1350 & 0.0152 & 0.0149 & 0.0165 & 0.0158 \\
\hline$\sim 1413$ & 0.0150 & 0.0144 & 0.0162 & 0.0157 \\
\hline \multirow[t]{4}{*}{1450} & 0.0145 & 0.0149 & 0.0149 & 0.0157 \\
\hline & \multicolumn{4}{|c|}{ Accel 4/Accel 1} \\
\hline & \multicolumn{4}{|c|}{250 PSI } \\
\hline & \multicolumn{2}{|c|}{ EJ ON } & \multicolumn{2}{|c|}{ EJ OFF } \\
\hline RPM & Upper & Lower & Upper & Lower \\
\hline 1350 & 0.0202 & 0.0193 & 0.0230 & 0.0217 \\
\hline$\sim 1413$ & 0.0161 & 0.0166 & 0.0182 & 0.0166 \\
\hline 1450 & 0.0166 & 0.0154 & 0.0166 & 0.0166 \\
\hline
\end{tabular}


Table 23. Effect of Expansion Joint on Vibration - $1822 \mathrm{ft}$ Test bed, Axis 2 (Outlet/lnlet)

\begin{tabular}{|c|c|c|c|c|}
\hline & \multicolumn{4}{|c|}{150 PSI } \\
\hline & \multicolumn{2}{|c|}{ EJ ON } & \multicolumn{2}{c|}{ EJ OFF } \\
\hline RPM & Upper & Lower & Upper & Lower \\
\hline 1350 & 0.048 & 0.055 & 0.061 & 0.064 \\
\hline$\sim 1413$ & 0.051 & 0.051 & 0.062 & 0.062 \\
\hline 1450 & 0.051 & 0.053 & 0.068 & 0.077 \\
\hline & \multicolumn{5}{|c|}{ Accel 5/Accel 2 } \\
\hline & \multicolumn{5}{|c|}{200 PSI } \\
\hline & EJ ON & EJ OFF \\
\hline RPM & Upper & Lower & Upper & Lower \\
\hline 1350 & 0.046 & 0.050 & 0.048 & 0.049 \\
\hline$\sim 1413$ & 0.053 & 0.051 & 0.043 & 0.044 \\
\hline 1450 & 0.053 & 0.057 & 0.049 & 0.050 \\
\hline & \multicolumn{5}{|c|}{ Accel 5/Accel 2 } \\
\hline & \multicolumn{5}{|c|}{250 PSI } \\
\hline EJ ON & Upper & Lower & Upper & Lower \\
\hline RPM & 0.058 & 0.058 & 0.054 & 0.058 \\
\hline 1350 & 0.057 & 0.055 & 0.049 & 0.048 \\
\hline$\sim 1413$ & 0.058 & 0.056 & 0.051 & 0.053 \\
\hline 1450 & \multicolumn{5}{|c|}{ EJ OFF } \\
\hline
\end{tabular}

Table 24 provides the total vibration ratios where the total vibration is determined as follows:

$$
\Omega_{\mathrm{Tot}}=\left(\Omega_{1}{ }^{2}+\Omega_{2}{ }^{2}\right)^{1 / 2} \text {. }
$$

For the 250 psi trials, cases with the expansion joint off have only slightly higher vibration ratios than trials with the expansion joint on. In general, the ratios are very similar for all trials, indicating there is no trend with respect to the engine RPM or operating pressure.

Table 24. Effect of Expansion Joint on Total Vibration - $1822 \mathrm{ft}$ Test bed

\begin{tabular}{|c|c|c|c|c|c|c|}
\hline & \multicolumn{4}{|c|}{ Total Vibration at Outlet/Total Vibration at Inlet } \\
\hline & \multicolumn{2}{|c|}{150 PSI } & \multicolumn{2}{c|}{200 PSI } & \multicolumn{2}{c|}{250 PSI } \\
\hline RPM & EJ ON & EJ OFF & EJ ON & EJ OFF & EJ ON & EJ OFF \\
\hline 1350 & 0.018 & 0.015 & 0.019 & 0.018 & 0.015 & 0.019 \\
\hline$\sim 1413$ & 0.017 & 0.015 & 0.016 & 0.017 & 0.015 & 0.016 \\
\hline 1450 & 0.016 & 0.015 & 0.016 & 0.016 & 0.015 & 0.016 \\
\hline
\end{tabular}




\section{Effect of Reducer on Pipe Vibration}

In this section, the effects of a set of two 3-to- 2 inch reducers are investigated on the $310 \mathrm{ft}$ test bed for the parametric testing. As mentioned previously, the reducer was placed just upstream of the expansion joint. Table 25 shows the vibration ratios for each of the accelerometers and for the total vibrations for the $200 \mathrm{psi}$ operating pressure. The trials with the reducer removed have a slightly lower vibration ratio than the ones with the reducer installed. This suggests that the restriction induced by the reducer caused additional vibrations near the blockage. For this set of data, as the pump RPM is increased, the vibration ratios also increased.

Table 25. Effect of Reducer on Vibration - $310 \mathrm{ft}$ Test bed, $200 \mathrm{psi}$

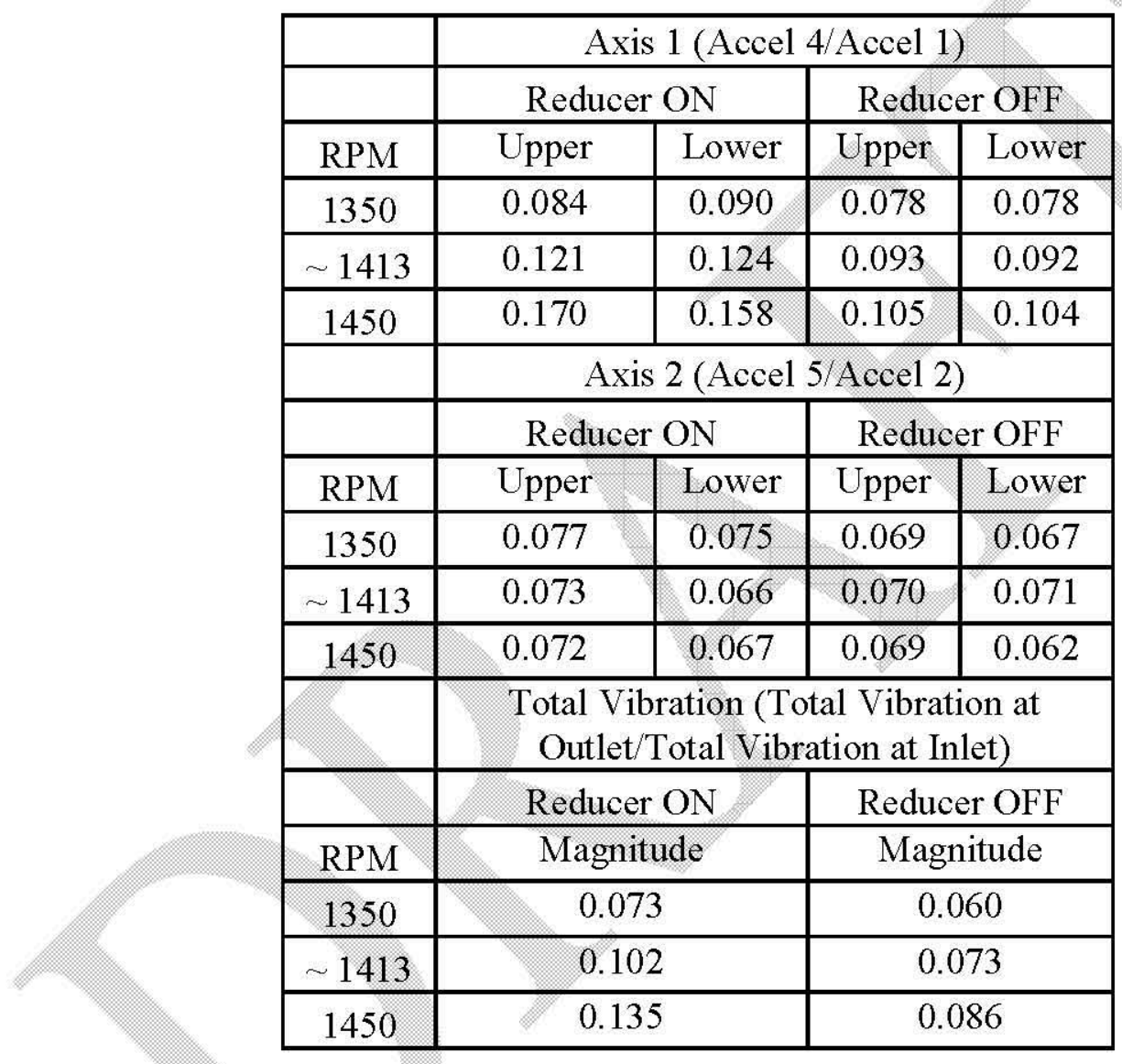

\section{FREQUENCY ANALYSIS}

In this section the pressure and acceleration data recorded during the trials were used to analyze the signals in frequency domain. The purpose of this analysis is to determine the range of frequencies in which AIMMS's Hydrokinetics Techonolgies operates. Similar to the analysis presented regarding pressure and vibration atenuation, this study was divided into three sections: flooding zone, target zone and parameteric trials (similar in nature to the target zone). For each section, the procedure of analysis required transfering the data from the time domain to the frequency domain using Fast Fourier Transform (FFT).

Figure 43 shows a typical result from the testing trials which provides pressure at the inlet of the pipeline in time domain together with its corresponding FFT. From the frequency domain, it is 
possible to depict the dominating frequiencies by identifying the peaks that show the larger amplitudes. In this study a dominating frequency is considered to have a value exceeding five times the signal's mean amplitude in the frequency domain.
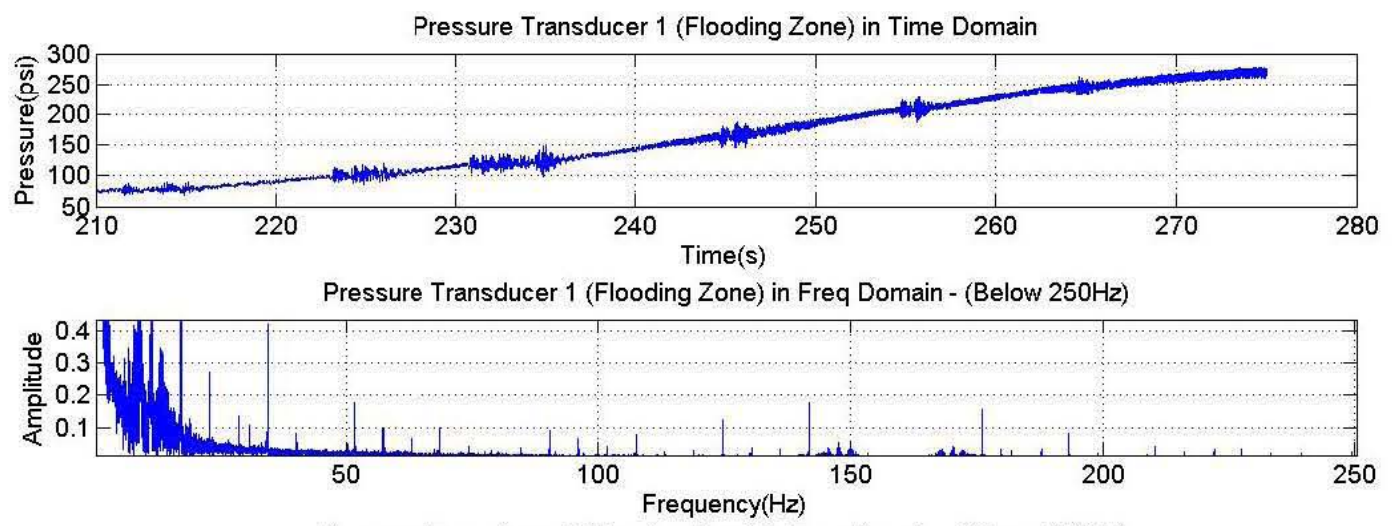

Pressure Transducer 1 (Fooding Zone) in Freq Domain - (Above 250Hz)

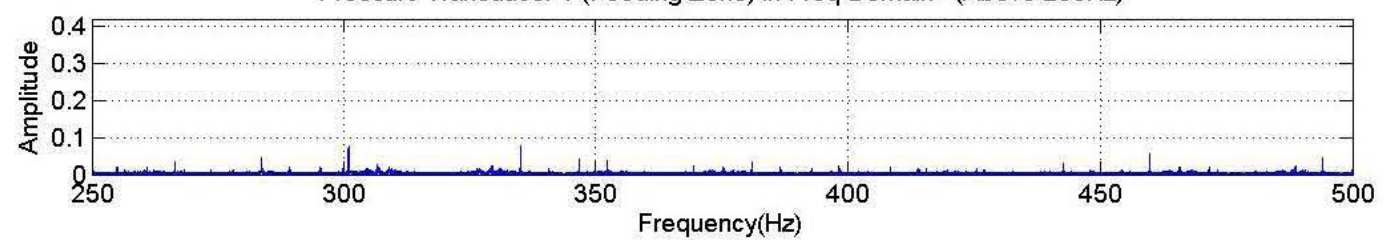

Figure 43. Signal from pressure transducer 1 in time and frequency domain.

After converting each of the six signals (one for each pressure transducer) to the frequency domain, the dominating frequencies were extracted for comparison. Table 26 shows the dominating frequencies found for the flooding zone during unplugging trials with an $8 \mathrm{ft}$ Bentonite plug on the $310 \mathrm{ft}$ test bed. As shown in Table 26, the frequencies observed at the plug location were common to all the frequency sets that were detected at the other locations. These frequencies are expected to play a major role in unplugging the blockages.

Using the data obtained from each trial, it was possible to characterize the frequency response of the system for the flooding and target pressure zones and the parametric trials. Figure 44 shows all the common frequencies registered by the pressure transducers for each trial during the flooding zone. Each vertical band represents one trial with its corresponding frequencies on the vertical axis.

During the flooding zone, it is clear that the dominating frequencies for all tests remained below $10 \mathrm{~Hz}$. This region is characterized by a steep increase in pressure with no movement of the blockage. The position of the valve from the AIMM's console was open in order to allow water to flow into the pipeline. By having the valve open, it also allowed any frequencies resulting from external sources (i.e. the rotary pump) to be registered. In some trials pulses were applied during the flooding zone. The applied pulses were random and of extremely low frequency $(<$ $0.01 \mathrm{~Hz}$ ). This suggests that the frequencies registered during the flooding zone are not associated with pulsing generated from the AIMM's operator. 
Table 26. List of Frequencies from the FFT

\begin{tabular}{|c|c|c|c|c|c|c|}
\hline P1 & P2 & P3 & P4 & P5 & P6 & $\begin{array}{c}\text { Common } \\
\text { Frequencies }\end{array}$ \\
\hline $1 \mathrm{~Hz}$ & $1 \mathrm{~Hz}$ & $1 \mathrm{~Hz}$ & $1 \mathrm{~Hz}$ & $1 \mathrm{~Hz}$ & $1 \mathrm{~Hz}$ & $1 \mathrm{~Hz}$ \\
\hline $2 \mathrm{~Hz}$ & $2 \mathrm{~Hz}$ & $2 \mathrm{~Hz}$ & $2 \mathrm{~Hz}$ & $2 \mathrm{~Hz}$ & $2 \mathrm{~Hz}$ & $2 \mathrm{~Hz}$ \\
\hline $3 \mathrm{~Hz}$ & $3 \mathrm{~Hz}$ & $3 \mathrm{~Hz}$ & $3 \mathrm{~Hz}$ & $3 \mathrm{~Hz}$ & $3 \mathrm{~Hz}$ & $3 \mathrm{~Hz}$ \\
\hline $4 \mathrm{~Hz}$ & $4 \mathrm{~Hz}$ & $4 \mathrm{~Hz}$ & $4 \mathrm{~Hz}$ & $4 \mathrm{~Hz}$ & $4 \mathrm{~Hz}$ & $4 \mathrm{~Hz}$ \\
\hline $5 \mathrm{~Hz}$ & $5 \mathrm{~Hz}$ & $5 \mathrm{~Hz}$ & $5 \mathrm{~Hz}$ & $5 \mathrm{~Hz}$ & $5 \mathrm{~Hz}$ & $5 \mathrm{~Hz}$ \\
\hline $6 \mathrm{~Hz}$ & $6 \mathrm{~Hz}$ & $6 \mathrm{~Hz}$ & $6 \mathrm{~Hz}$ & $6 \mathrm{~Hz}$ & $6 \mathrm{~Hz}$ & $6 \mathrm{~Hz}$ \\
\hline $7 \mathrm{~Hz}$ & $7 \mathrm{~Hz}$ & $7 \mathrm{~Hz}$ & $7 \mathrm{~Hz}$ & $7 \mathrm{~Hz}$ & $7 \mathrm{~Hz}$ & $7 \mathrm{~Hz}$ \\
\hline $8 \mathrm{~Hz}$ & $8 \mathrm{~Hz}$ & $8 \mathrm{~Hz}$ & $8 \mathrm{~Hz}$ & $8 \mathrm{~Hz}$ & $8 \mathrm{~Hz}$ & $8 \mathrm{~Hz}$ \\
\hline $9 \mathrm{~Hz}$ & $9 \mathrm{~Hz}$ & $9 \mathrm{~Hz}$ & $9 \mathrm{~Hz}$ & $9 \mathrm{~Hz}$ & $9 \mathrm{~Hz}$ & $9 \mathrm{~Hz}$ \\
\hline $10 \mathrm{~Hz}$ & $10 \mathrm{~Hz}$ & $10 \mathrm{~Hz}$ & $17 \mathrm{~Hz}$ & $17 \mathrm{~Hz}$ & $31 \mathrm{~Hz}$ & \\
\hline $11 \mathrm{~Hz}$ & $11 \mathrm{~Hz}$ & $11 \mathrm{~Hz}$ & $31 \mathrm{~Hz}$ & $31 \mathrm{~Hz}$ & & \\
\hline $12 \mathrm{~Hz}$ & $12 \mathrm{~Hz}$ & $12 \mathrm{~Hz}$ & $60 \mathrm{~Hz}$ & $60 \mathrm{~Hz}$ & & \\
\hline $13 \mathrm{~Hz}$ & $13 \mathrm{~Hz}$ & $13 \mathrm{~Hz}$ & & & & \\
\hline $14 \mathrm{~Hz}$ & $14 \mathrm{~Hz}$ & $14 \mathrm{~Hz}$ & & & & \\
\hline $15 \mathrm{~Hz}$ & $15 \mathrm{~Hz}$ & $15 \mathrm{~Hz}$ & & & & \\
\hline $16 \mathrm{~Hz}$ & $16 \mathrm{~Hz}$ & $16 \mathrm{~Hz}$ & & & & \\
\hline $17 \mathrm{~Hz}$ & $17 \mathrm{~Hz}$ & $17 \mathrm{~Hz}$ & & & & \\
\hline $19 \mathrm{~Hz}$ & $23 \mathrm{~Hz}$ & $23 \mathrm{~Hz}$ & & & & \\
\hline $23 \mathrm{~Hz}$ & $34 \mathrm{~Hz}$ & $29 \mathrm{~Hz}$ & & & & \\
\hline $29 \mathrm{~Hz}$ & & $31 \mathrm{~Hz}$ & & & & \\
\hline $31 \mathrm{~Hz}$ & & $34 \mathrm{~Hz}$ & & & & \\
\hline $34 \mathrm{~Hz}$ & & & & & & \\
\hline $51 \mathrm{~Hz}$ & & & & & & \\
\hline $125 \mathrm{~Hz}$ & & & & & & \\
\hline $142 \mathrm{~Hz}$ & & & & & & \\
\hline $176 \mathrm{~Hz}$ & & & & & & \\
\hline
\end{tabular}

Figure 45 shows the frequencies present during the target pressure zone testing. Again, each vertical band corresponds to a trial having its corresponding frequencies on the vertical axis. In contrast to results for the flooding zone, it is clear that the driving frequencies show an increase in frequency range to approximately $35 \mathrm{~Hz}$. The position of the AIMM's control valve during this region was opened and closed randomly, depending on the operator input. This mode of operation of the system is reflected by the dissimilarity of frequency values recorded at each trial. 


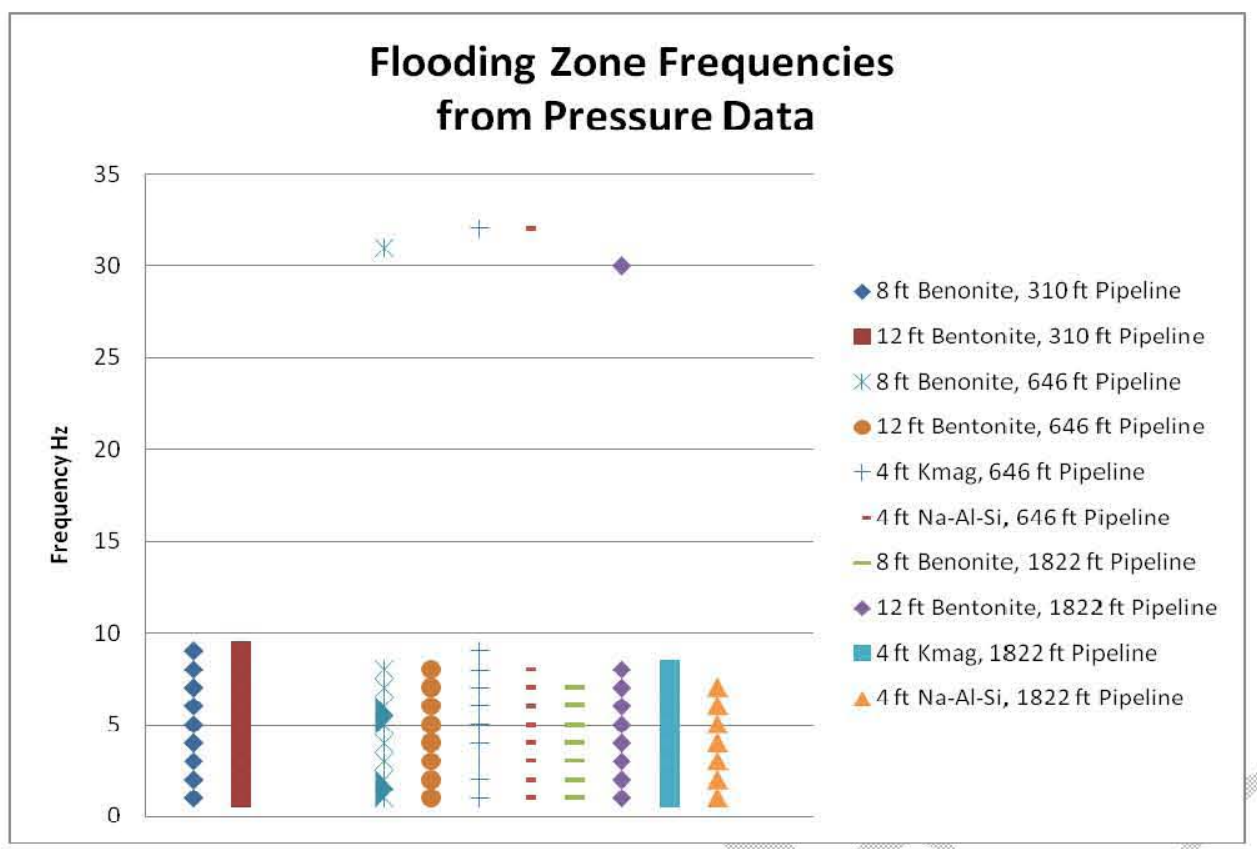

Figure 44. Frequencies present during the flooding zone.

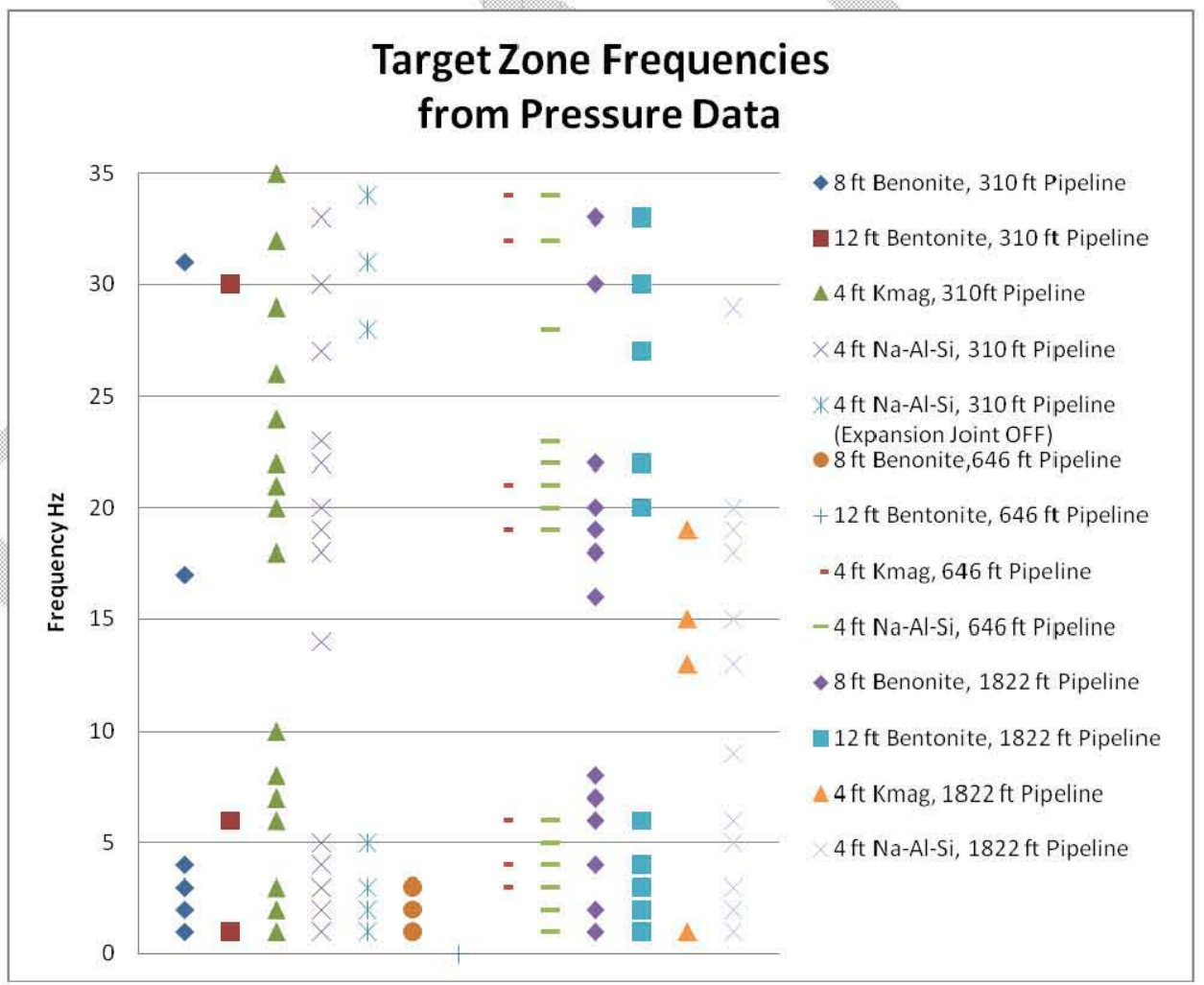

Figure 45. Frequencies present on the target zone.

Figure 46, Figure 47, and Figure 48 show the frequencies registered on the parametric trials for the $310,646,1822 \mathrm{ft}$ test beds, respectively. During these trials, the operator was instructed to perform the opening and closing of the valve at a frequency of 80 beats per minute $(1.33 \mathrm{~Hz})$ by 
following a metronome. However, since this frequency value lies within the range of external sources it did not register as an independent frequency.

In the flooding and target pressure zones, the parametric trials show dominating frequencies at or below $35 \mathrm{~Hz}$. A difference on the pipeline length shows differences in the frequencies captured. Note that as the pipeline length increases, frequencies below $10 \mathrm{~Hz}$ decrease in number. It was observed that an increase in pipeline length reduced the number of frequencies translated from the inlet to the plug section that assist in the unplugging process.

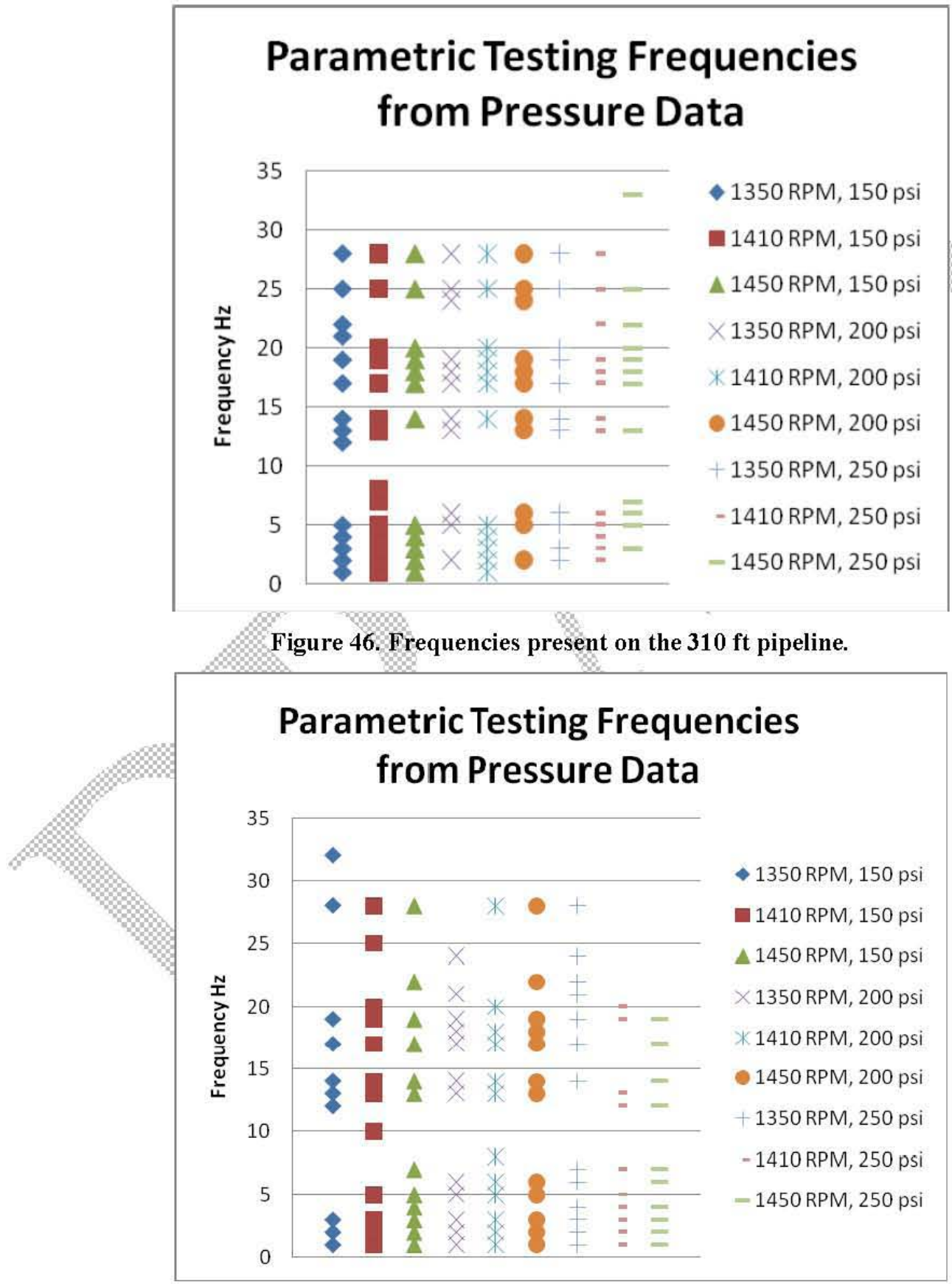

Figure 47. Frequencies present on the $646 \mathrm{ft}$ pipeline. 


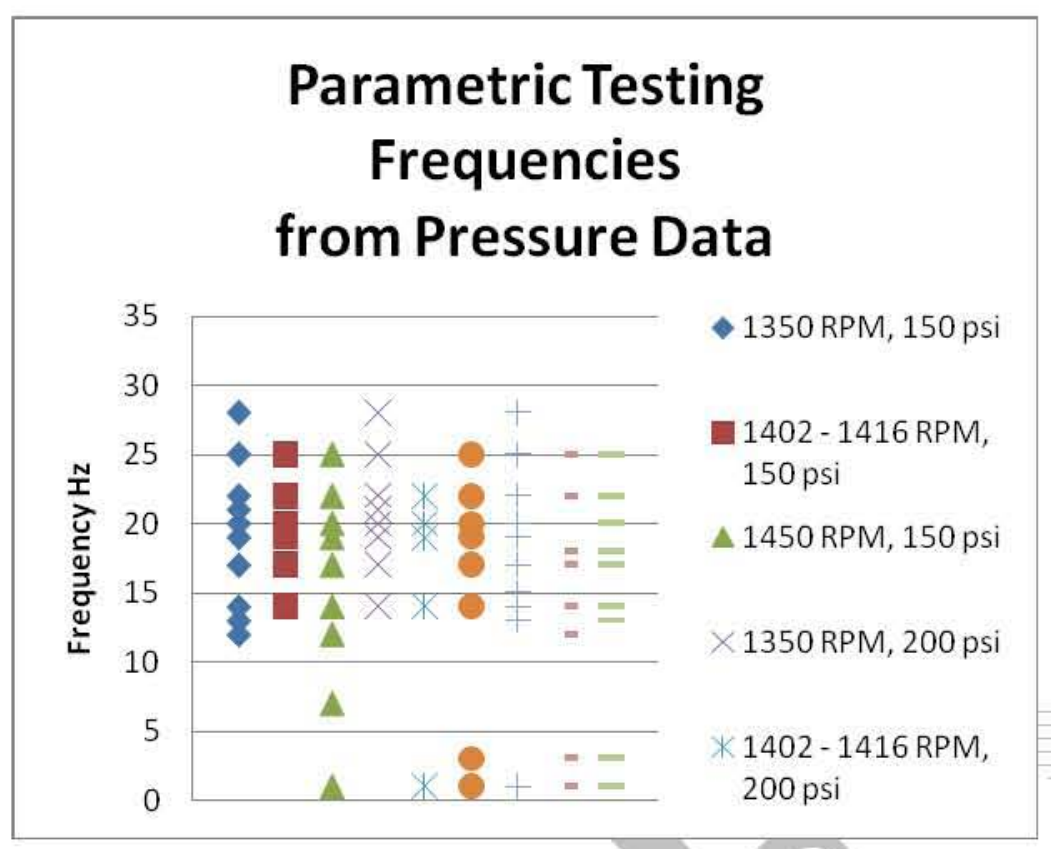

Figure 48. Frequencies present on the $1822 \mathrm{ft}$ pipeline.

\section{CONCLUSIONS}

AIMM Technologies Hydrokinetics process was evaluated using an instrumented test bed at three pipeline lengths with three types of blockages. Tests trials were conducted to provide an understanding of the underlying principles of how the unblocking technology functioned. Pressure pulses were created at the inlet of the pipeline by opening and closing valves on a pressurized manifold in AIMM's control box. The pipeline was not vacated of air prior to filling, so the pipeline consisted of both air and water. The amount of air in the pipeline line was not monitored. In addition, the testing trials were conducted with a restricted pressure limit in the pipeline of $300 \mathrm{psi}$. This limit is a safety requirement for HLW cross site lines at the DOE.

During the testing trials, AIMM Technologies was more successful removing the Bentonite plugs than the salt-based plugs. During the final test trials, AIMMs was able to remove 5 of the 6 Bentonite plugs. AIMMs did successfully unplug one Na-Al-Si plug during the commission trials but could not unplug three others during the final trials. They were able to partially get flow through a $4 \mathrm{ft} \mathrm{Kmag} \mathrm{plug} \mathrm{at} \mathrm{the} 310 \mathrm{ft}$ test bed, but could not remove the Kmag at the other pipeline lengths. It should be noted that the Bentonite plugs required lower extrusion pressures than the salt-based plugs.

In general, the data from the unplugging and parametric trials showed that the pressure pulses attenuated significantly from the inlet to the blockage section during the unplugging process. The vibrations were also attenuated significantly from the inlet to the blockage during the unplugging process. This suggests that the remaining air within the pipeline and the low operating pressure inhibited the ability of AIMM's Hydrokinetics process to operate at its optimum capability. The variability of the remaining air in the pipeline at different lengths affected the data analysis 
adversely in that trends for pressure and vibration propagation were difficult to identify in some trials.

The significant attenuation of the pressure pulses and vibration suggest that the mechanism for removing the blockages on the successful trials was a combination of the static pressure and the small residual pressure pulses propagated to the blockage. Imposing a vacuum on the pipeline prior to filling would significantly decrease the attenuation rate and improve performance.

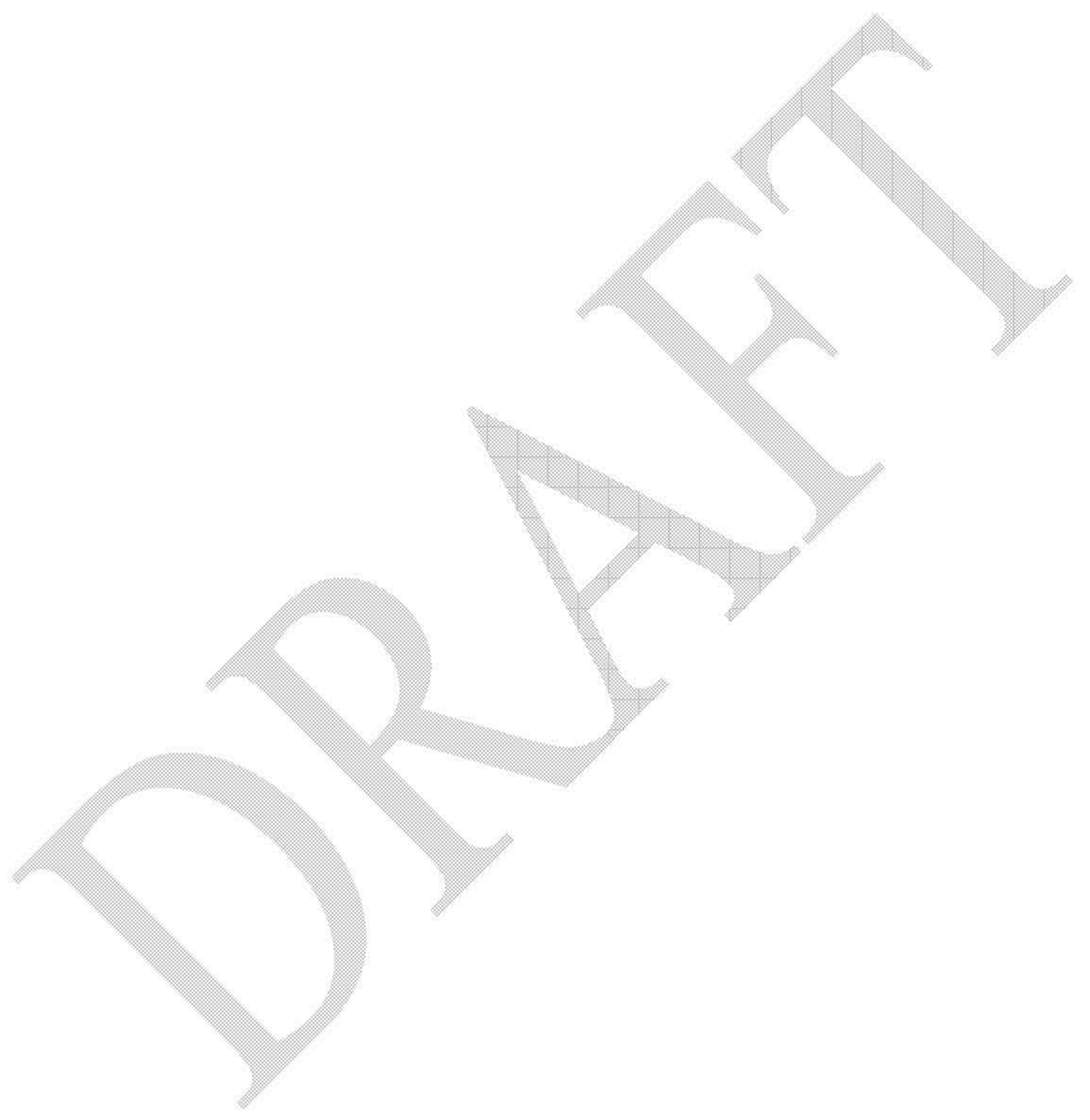




\section{REFERENCES}

Garcia, P. M., (1997). U.S. Patent No. 5,674,323. Washington, DC: U.S. Patent and Trademark Office.

Golcar, G. R., J. R. Bontha, J. G. Darab, M. R. Powell, P. A. Smith, and J. Zhang., "Retrieval Process Development and Enhancements", Project Fiscal Year 1995 Simulant Development Technology Task Progress Report, PNNL-11103, Pacific Northwest National Laboratory, Richland, Washington (1997).

Powell, M.R., Golcar, G.R., Geeting, J.G.H., "Retrieval Process Development and Enhancements Waste Simulant Compositions and Defensibility", PNNL-11685, Pacific Northwest National Laboratory, Richland, Washington (1997).

Powell, M.R., "Initial ACTR Retrieval Technology Evaluation Test Material Recommendations", PNNL-11021, Pacific Northwest National Laboratory, Richland, Washington (1996). 
RPP-RPT-53783, Rev. 0

\section{Appendix C}

\section{AIMM ARTICLE, "A BETTER WAY TO CLEAN"}




\section{A Better

\section{A patented tube cleaning process called Hydrokinetics hess helped AlMM Technologies build a business domestically and overseass}

\section{By Garol Brzozowshi-Gardher}

$\mathrm{H}$ idioblasting and chemical cleaning are the dominant methods or industrial cleaning. But RlMM Technologies, a service company in Lallarque Texas, has used a patented utrasonicbased cleaning process to make headway in the Un ied Gates market as yell as internationally.

RIMM manu factures the Hod ick inet is equipment for is ow use. The process uses sonic resonance w wh water to clean pipes and tubes. Most clients are in the petrochemical field; the batance are in goremment services and food prooessing.

Brocks Bradion, RIMM president, bought the company five years ago fiom Raph and Pa Garia, who stated $x$ in 1991 and patented the Hydrckinetic prooess (Ralph was the ime ntooj. Since
Brooks took over, the company has expanded theprocess, receined asecond patent, and has a thind patent pending. Company revenues have troled, and klMM has gone global. Besides operaing offices in Texas, Lousiana and Monterify, Mexico (where Mizuel Monet s coun ntry managen, slm M partne is with companies in Morway, the United Kingdom and Saudi Rabia.

knother part ner, The Attantic Group, povides service to the US. Nays which added Hydrckinetics to its technical manual this year, qening the doors for exvicing the lvesse $\mathrm{k}$ world wide.

'The Hydrokinetics process is impressive fiom several viewpoints, but the thing that impresed me in rially was is safty" Brooks says. "At's in herently sak, and in the industral cleaning industry, saky is paramount. There is no
"The Hydrolinetica proceses is impresgive from soveral viowpoints, but the thing that impreased mo initially was its sofety. It 9 inferently sake, and in the industrial cleaning industry sefoty is peramount. There is no procese we know of that comes close as fer a being sefe for the operator end for the environment."

\section{Brodks Bradford}
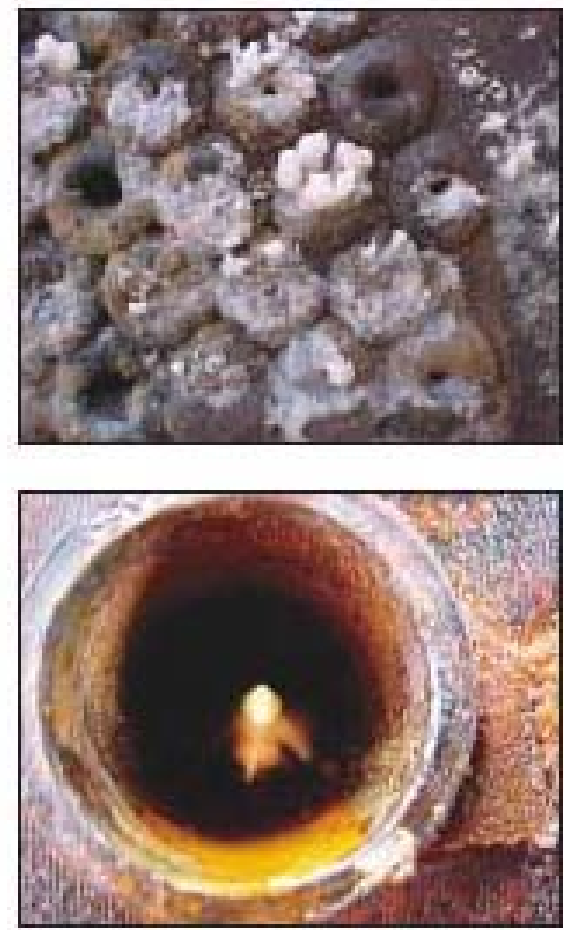

Upper photo, heat exchanger tubes before Hydrokinetic cleaning; lower photo, entry to tubes after $\mathrm{H} y d r o k i n e t i c$ cleaning. process we know of that comes close as far asbeing sale for the operar and for the envion ment."

\section{Shaking it loose}

In Hidrokinetics, the tite or pipe to be cleaned is first filled whi an cosiltaing weterstieambesed on regonant fiequency. Once flow is achieved, a bullet is introduced in the tube to maintain the water column. The tube is then refilled whth water. The water (or sonic) resonance is transteried through the water column.

The buling and the tube reonate at different fiequencies, breaking the bond bet ween them, allow ing the foul ing to be easiby expelled out of the cpposite end of the tute. The buling is expellad in a anakel the fashion ather than in particles. Add ionally, the material comes out the end coposite the operar.

The equiment consists of a hydraulic moniboring devioe in a sealed cabinet. Wh the arailabilky of a comentional plunger pump ( 0,000 pei at 20 gpm) and ton psi service air, tech nician and unican periorn clean ing any where.

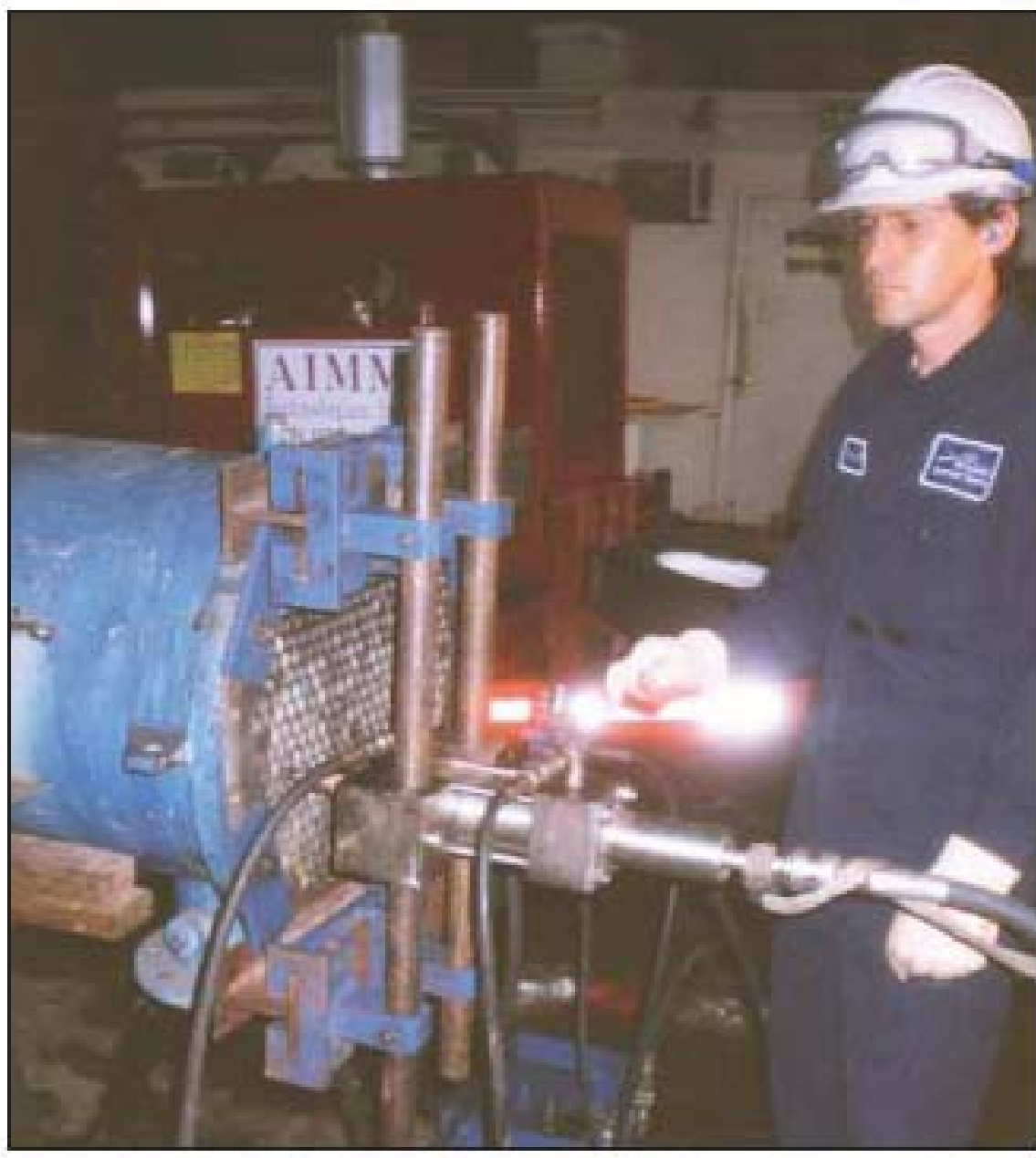

AlMM employee Ame rico Almeich ope rates a Hydrokinetic ra m zase mbly. Hydrokinetics is a new tube clean ing tech nobgy devebped by AlMM, based in LaMarque, Texas.

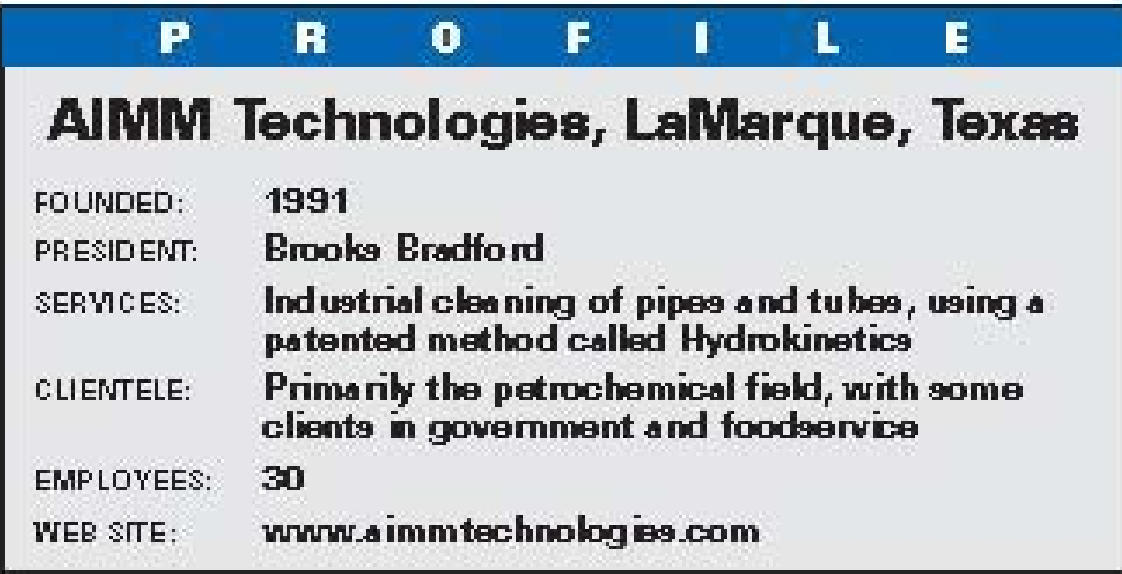

Biocks, who ow ned a rental company providing re fige at ion services be bre he aoquired AlMm, bought the clean ine company because he was impresed whth the Hudickinetios method and is bw ratio of employees to income. He also likes the fact that the method porduces immediate results. Dexple is emphasis on Hydrckinetics, AMMM still does tradi tional cleaning. The company oflers hidictlating up to $36,000 \mathrm{pEi}$ for indus tral clean ing orsu iface prepartion.

While safeby is a prime concem in any industry, $*$ is even more so in 
"rit wots them back into prodication faster, and that's where they make their money These plents work on soveral hundred thousend' dollers an hour and if you can bring them back into production 5 to fo hours faster, then you've done a pood job for them."

Ralph Garcia chemical plants, where $\mathrm{AlMM}$ does much of its woik. AMM counts on Hydrokinetios to be sak but abo has developed a sieky piceram that is appored by all clients and is adhered to dails, says Gary Dun n, heath, sakty and emvion ment (HSE) manager.

\section{No bst-time aceidents}

In AIMM's 10 years, the company has never had a lot time accident. Clients industrial plant safecy engineers and supervisors appreciate that recont. Bonald Cotbon, RIMM operations supervi sor, attributes the salety iecond to the frt that Hodrokinetios does nok expose the operator to high-pressure water or wote materals In addrion to sakty, RIMM vicepresident Raph Garia faors Hodickinetios br.

- Tme axinga The method is 40

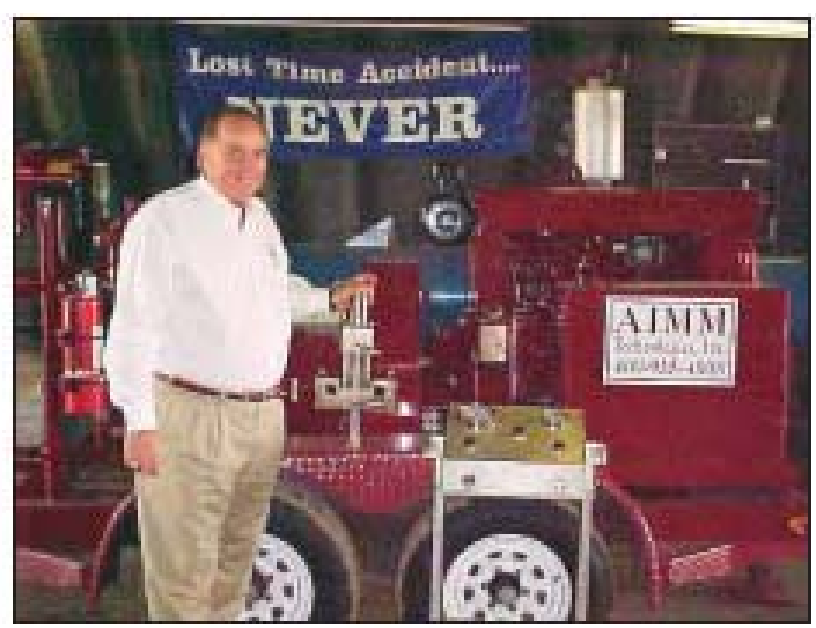
Hud ick inet is? Some question whether $\mathrm{Hy}$ dokinetics streses tuber and poing. The company says the maximum fiequency of 11,250 vbrations per minute is er below the number of cycles that would caus trigue in even the soltes metak.

For some $00 \mathrm{~m}$ panies, cost may be a factor. kntone Bekher, operations manager, concedes that Hydrokinetics

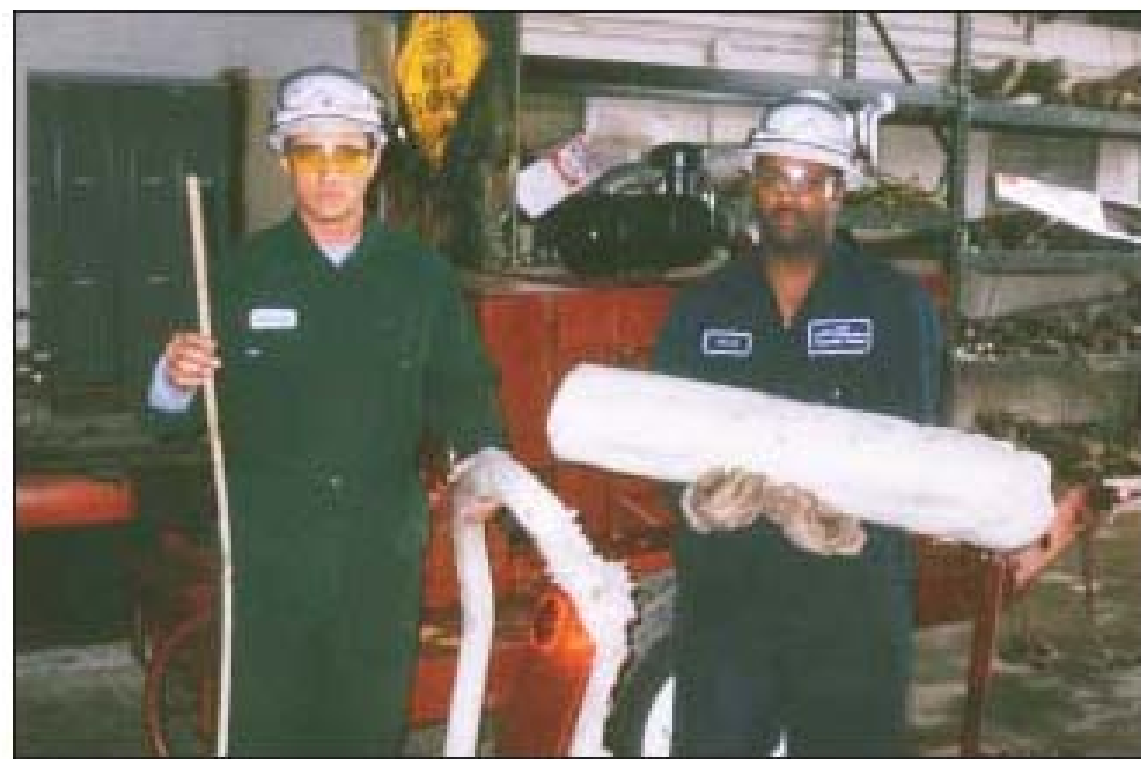

Upper photo, B rooks Bradtord, president of AlMM Tech nologies, shows the equipment used for the companys patented Hydrokinetics process. Lover photo, technicians Domingo Bla nco [left] and Mark Guidry display bulant re moved from tubes.

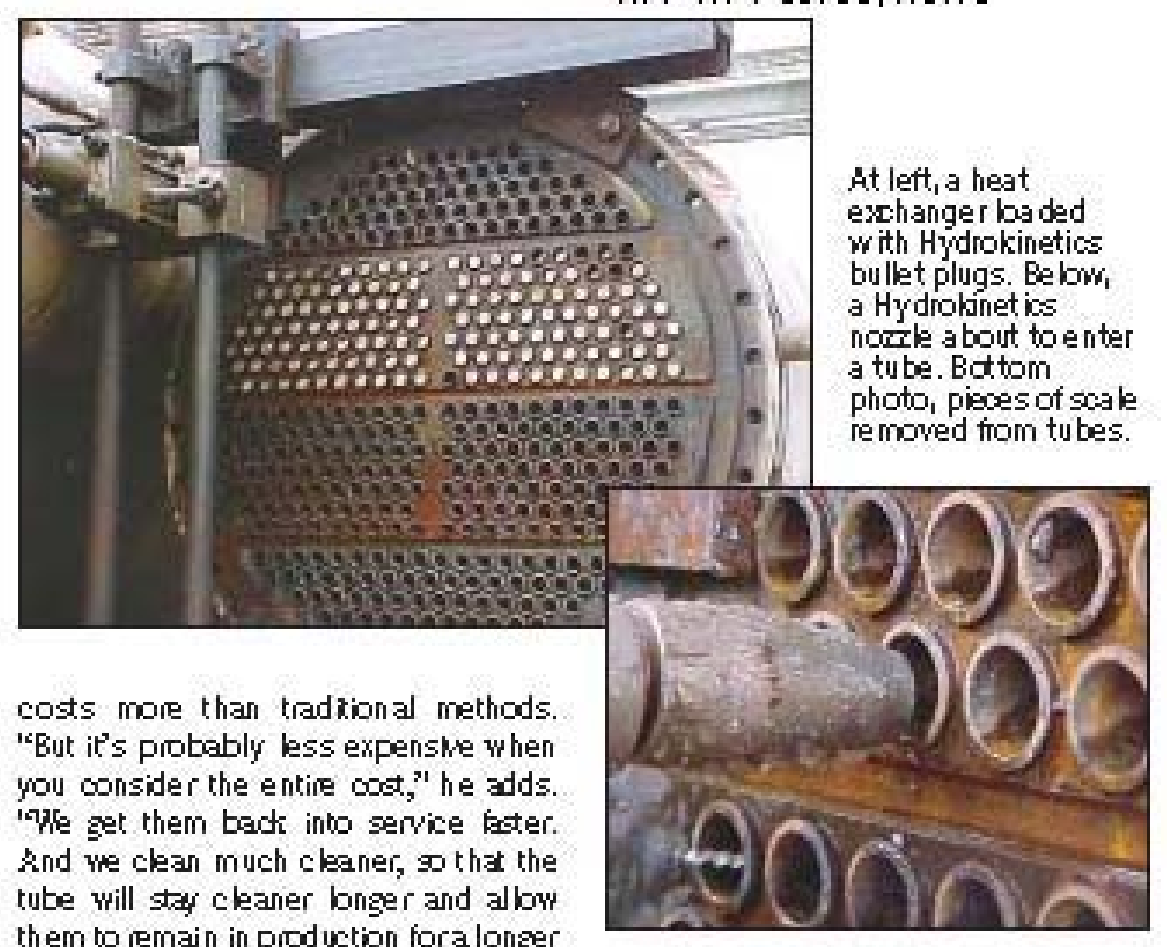

them to remain in production fora longer period. When you consider all those th ings, we're piobsbly cheaper."

\section{0 pertent success}

Company officials say Hydndzinetics cleaning is successful in more than 90 percent of amlications. $t$ oten woks best whene othertiad zional methods fil, such $a$ in Ushaped piping or tubing, confined wokspaces and lines that ane completely blocked.

'Shnytody who says ther'ie 100 percent on any job might be hedging a litk bx," sass Biocks. "Whe're not 00 percent, I don't know that anyone $i s$. But wath things that give us a problem, they'ie

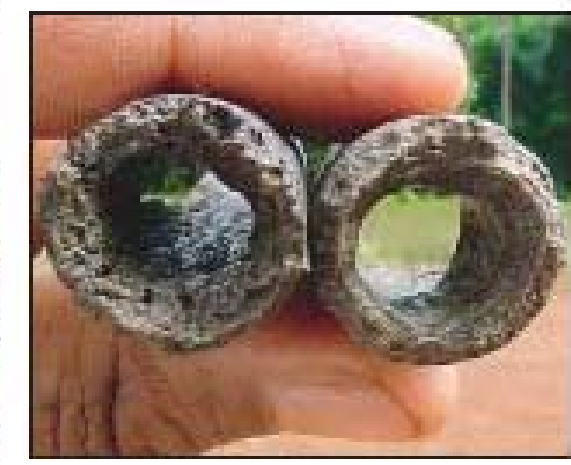

impossble for other people." kst Brocks to che his company's

\section{Going Global}

The re are cultural corsit ratione uhen a compary takes a gbbal prese noe. Dut of respectfor the boalcultures ove rseas, AlMM Technologies educates its employees on the boalcustome through inthouse or outsourced ta ining.

'The e's a wast dife rence betweengoing o England or Normay we rsus going b Saudi Arabia," says Brooks Bradtord, Alliti"s presidert. The amourt of instructione mployees receive de pends on whe re they are going and how bong they willstay.

For exa mple, Antone Be bher, operations manager, recently complated a fivemonth project in Central Africa in an area with few roads, where most travel was by a ir or water. 'His a result, yourve got to learn o be patientand make do with whatyou have," he says. "You karn to work with the boal population not against it The ir morkdsys and their attitudes are different."

"You have to be careful into you send on a project like that, as to inhether they canstand the type of pessure that comes about both daiky and after they've been there for 30 days. If they're off in the jungles, can they handle that isolation? Not mary pecole can do it."

Productivity thes a rosedive after a bout three weeks. Conseque ntty, ma nagers rotate employees in and out of international projects on a thee or bufmee $\mathrm{K}$ basis. While Brooks tries be mpty boal people, he sends a n Allillil supe visor vevery jab. If the job requires more US. employees, he'llserd them. But tis goal is to have his alliance partre rs act inde pendertly with little input from the US. AllMill atioe. 


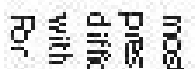

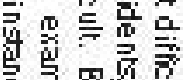 \\ 勇署觉

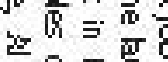

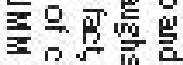

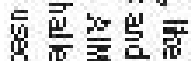

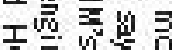

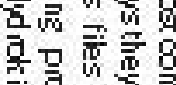

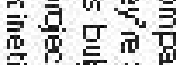

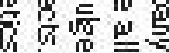

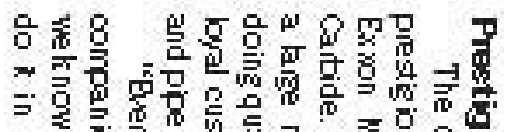
w

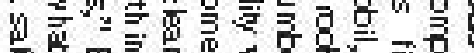
5.

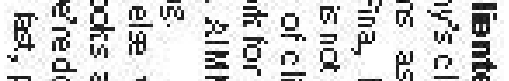

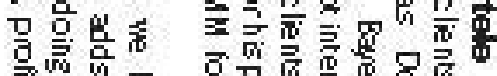

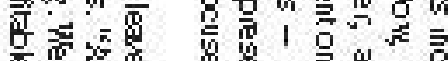
ว

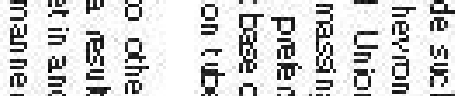

恶要

弯离部。

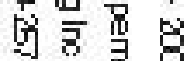

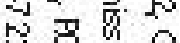

(5. 8

政

每

表罚志

的

要希

娄票

罗查

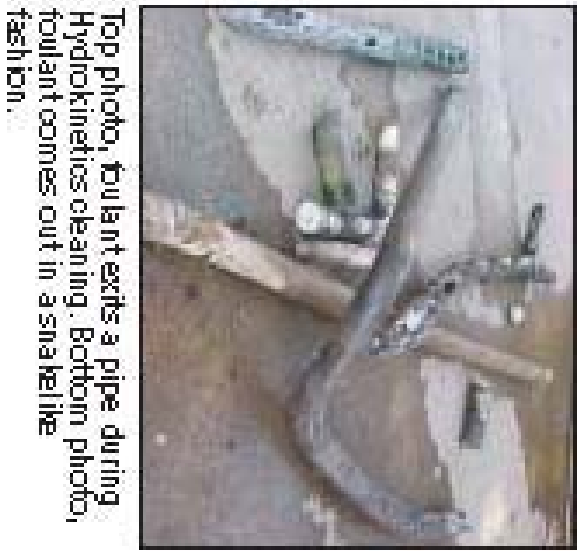

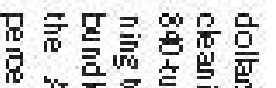

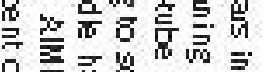

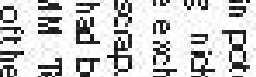

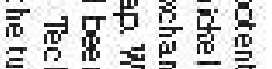

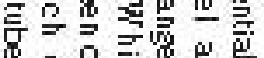

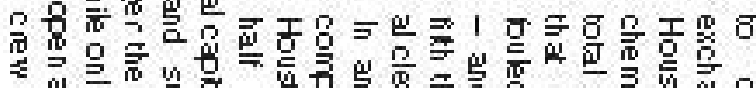

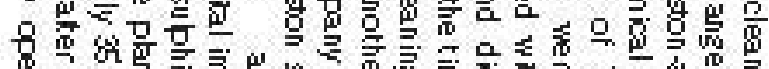

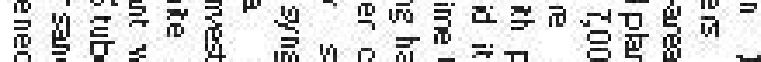

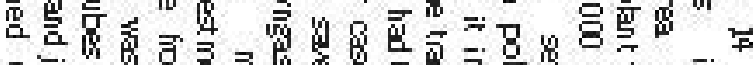

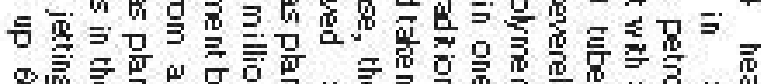

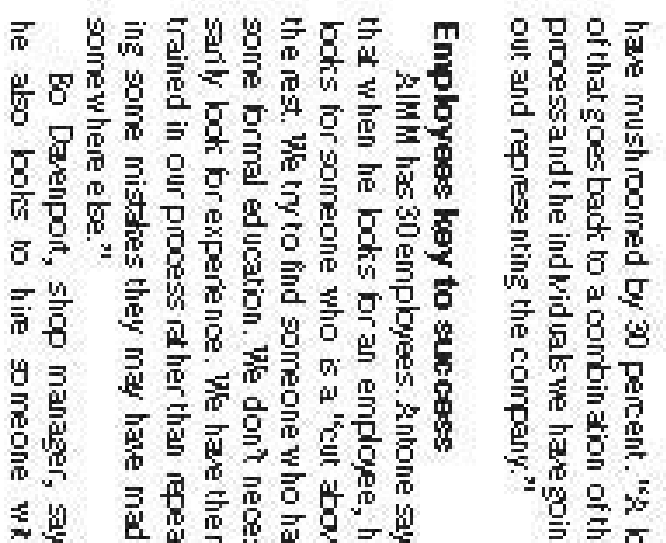

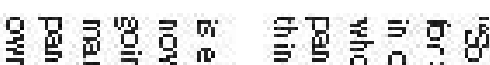

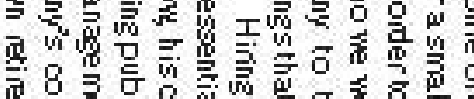

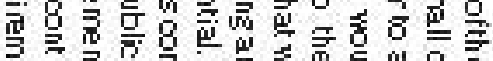

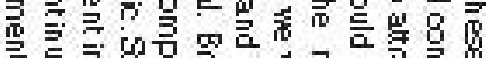

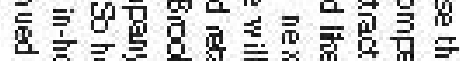

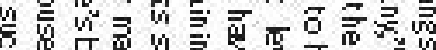

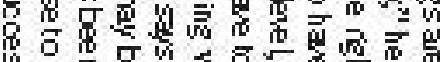

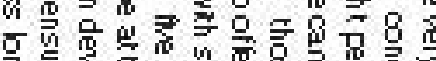

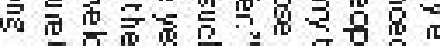

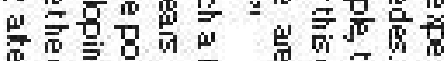

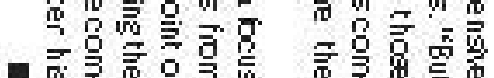

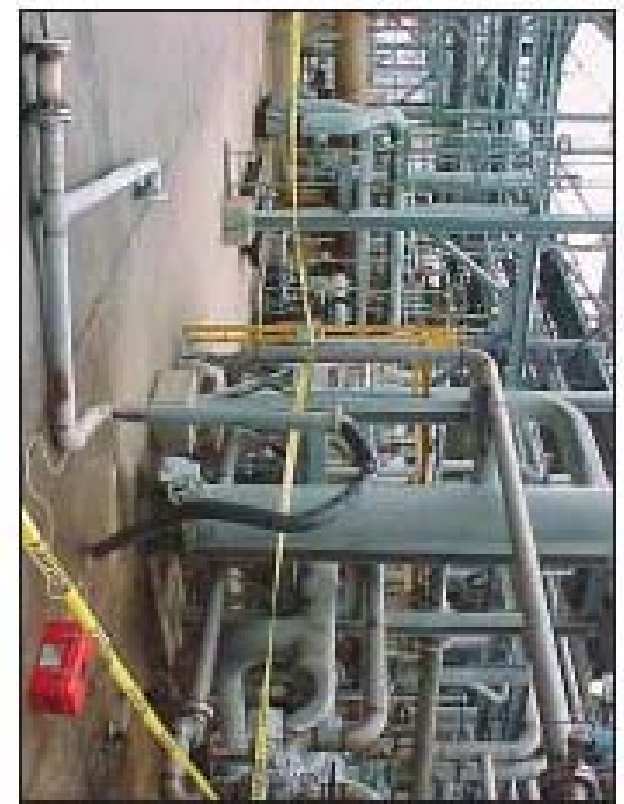

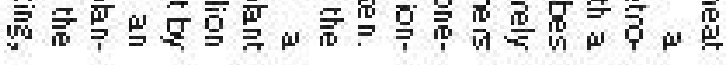

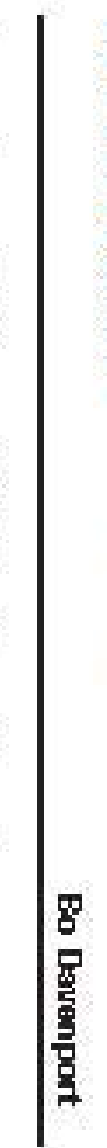

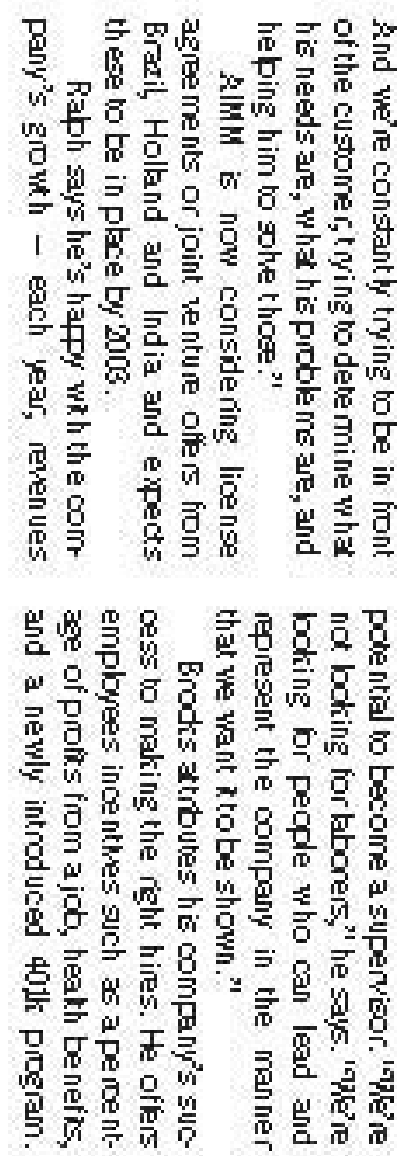




\section{Appendix D}

PIPELINE UNPLUGGING ASSESSMENT AND RECOMMENDATIONS FOR THE FERNALD ENVIRONMENTAL MANAGEMENT PROJECT

(SECTION 3.5 AND APPENDIX I) 


\title{
Pipeline Unplugging Assessment and Recommendations for the Fernald Environmental Management Project
}

\author{
M. I. Morris
}

J. L. Ladd-Lively

B. E Lewis

Nuclear Science and Technology Division

Oak Ridge National Laboratory

June 18, 2004 


\subsection{Hydrokinetics ${ }^{\mathrm{TM}}$}

The Hydrokinetic ${ }^{\mathrm{TM}}$ process is based on the induction of sonic resonance within a cleaning water stream. This sonic resonance travels through the water stream and transfers vibrations to both the pipe and the blockage, as diagramed in Fig. 7. A pig may also be used in conjunction with this technology and is shown in part 3 of Fig. 7 as an optional step in certain applications. The use of pig is not envisioned for the Fernald application. Because of the different compositions of the pipe wall and the blockage material, the blockage and the pipe wall vibrate at different frequencies, thus breaking the cohesive bond between them and allowing the blockage to be expelled from the pipe. By amplifying the pulsation with a high-pressure plunger pump, the water stream accelerates to achieve a velocity of $2,100 \mathrm{ft} / \mathrm{s}$. The generation of the sonic vibration takes a few milliseconds to complete, and the tube or pipe being cleared is exposed to the sonic wave for only a fraction of the process time. A maximum frequency of 11,250 vibrations per minute can be achieved, far below the number of cycles per second needed to cause metal fatigue in even soft metals such as copper-nickel alloys or copper. 


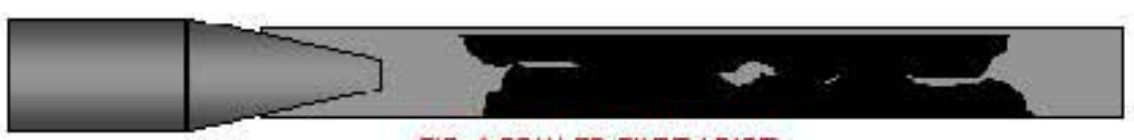

FIG. 1 FOULED TLBE / PIPE

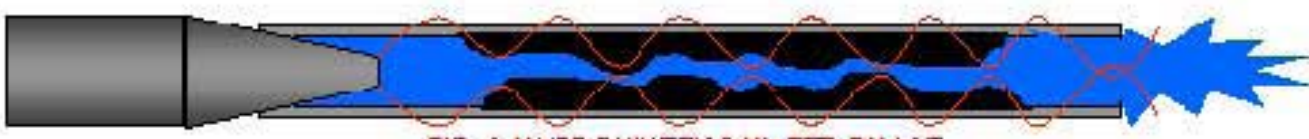

FIG. 2 H YOROKIVETICS WATER PHASE

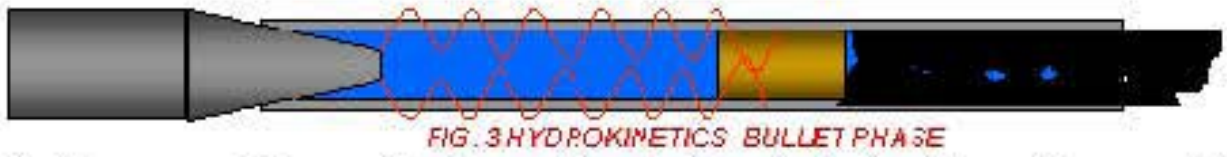

Fig. 7. Diagram of the application of hydrokinetic technology. Source: AIMM Technologies web site at http:/Wwwaimmtechnologies.com/

This process is unaffected by piping configuration or distance from plug. It can be operated remotely with minimal exposure. Process line fluid does not come in contact with equipment. It typically requires no system modification; hook up to single access point connection. Operating equipment is minimal; consisting of a pump skid and valve operating console. Operation is simple and mobilization/demobilization is quick and easy. Unplugging time is effected by distance from entry port but not length of plug. However, some plugs may require pressures up to 10,000 psi. Expected pressures in the Fernald application are in the range of 500 to $2,500 \mathrm{psi}$. This patented technology is presently avalable from only two vendors and has primarily been applied in the chemical, petrochemical, and public works industries.

The following vendors provide Hydrokinetics ${ }^{\text {TM }}$ service:
AIM Technologies
$11135^{\text {th }}$ Street South
Texas City, TX 77590
Phone: $409-945-5414$
Fax: $409-945-6022$
Email: www aimmtech@wt.net
Website: www aimmtechnologies.com
Contact(s): Brooks Bradford and Ralph Garcia
AIMM was visited and is discussed in detail later in the report.

Tube Cleaning Technologies

1480 East Highway 6

Alvin TX 97511

Phone: 281-842-1904

Fax: 281-842-1905

Contact: Danny Blackwell 


\title{
Appendix I: Completed Pipeline Unplugging Vendor Survey Forms for Recommended Technologies
}

\author{
Pipeline Unplugging Vendor Survey Form for Hydrokinetics ${ }^{\mathrm{TM}}$ Technology \\ Provider AIMM Technologies
}

\author{
Company Contact Information \\ AIMM Technologies \\ $11135^{\text {th }}$ Street South \\ Texas City, TX 77590 \\ Phone: 409-945-5414 \\ Fax: 409-945-6022 \\ Email: aimmtech@wt.net \\ Website: www.aimmtechnologies.com \\ Contact(s): Brooks Bradford, and Ralph Garcia \\ Process name: HYDROKINETICS ${ }^{\mathrm{TM}}$
}

Process description: The Hydrokinetic ${ }^{\mathrm{TM}}$ process is based on the induction of sonic resonance within a cleaning water stream. This sonic resonance travels through the water stream and transfers vibration to both the pipe and the blockage. Because of the different compositions of the pipe wall and the blockage material, the blockage and the pipe wall vibrate at different frequencies, thus breaking the cohesive bond between them and allowing the blockage to be expelled from the pipe. By amplifying the pulsation with a high-pressure plunger pump, the water stream accelerates to achieve a velocity of $2,100 \mathrm{ft} / \mathrm{s}$. The generation of the sonic vibration takes a few milliseconds to complete, and the tube or pipe being cleared is exposed to the sonic wave for only a fraction of the process time. A maximum frequency of 11,250 vibrations per minute can be achieved, far below the number of cycles per second needed to cause metal fatigue in even soft metals such as copper-nickel alloys or copper.

\section{Advantages:}

1. Unaffected by piping configuration or distance from plug.

2. Can be operated remotely, minimal exposure.

3. Non-intrusive, process line fluid does not come in contact with equipment

4. Typically requires no system modification; hook up to single access point connection.

5. Operating equipment minimal consisting of pump skid and valve operating console.

6. Operation simple

7. Quick and easy mobilization and demobilization.

8. Unplugging time effected by distance from entry port but not length of plug.

9. Manual or automated system. 
10. Commercially available.

\section{Disadvantages:}

1. For some plugs may require pressures up to 10,000 psi. Expected pressures in this application up to 2,500 psi.

2. Patented process only one other company found that may be able to supply this technology.

Company safety record: Zero recorded accidents for the company and the process. Worker's compensation Experience Modification Rate (EMR): 0.77 FY03

Failure rate and reasons for failures: Low pressure and/or small diameter piping where unplugging equipment could not operate at the needed pressure. Acrylic material type plugging also can cause problems

Company size (employees, sales): 30 employees (average), which fluctuates depending on work load. $\sim \$ 3$ Million (FY-03)

Number of years in business: 15

Insurance: Zurich North America

Industries served: Chemical, petrochemical and public works

List of clients and references: At end of report

Comparison with competing technologies: Hydroblasting, chemical cleaning, mechanical cleaning

Current projects: Ongoing services to the chemical and petrochemical industry

Experience working with DOE? Sites and Projects: FIU demonstration only.

Radioactive environment experience: None

Experience with various lengths, diameters, and materials of piping: Have processed the whole gambit of piping. Low pressure rated lines could hinder process.

Experience with plugs of various materials (i.e. $\mathrm{CaCO}_{3}$ ): Many different kinds

Maximum size of plugs cleared and time required: Up to $36-\mathrm{in}$. diam, and $100+\mathrm{ft}$ long plug. Time relative to the distance from the plug. A few minutes up to worst case of several days. 
Experience with closed systems and/or remote operations: System is especially effective in this environment - See advantages.

\section{Contracting services:}

Rent or lease: $\sim \$ 9-15 \mathrm{~K} / \mathrm{month}$ depending on length of lease.

Service on as needed basis: $\$ 20-50 \mathrm{~K}$

Purchase: Probably not. Available

Demonstration Cost: AIMM would perform at no cost (Provided if

demonstration was successful AIMM would be awarded a lease agreement)

System connections needed (size and type): Single flange - Variable size and type.

\section{Number of people required to operate: $2-3$}

Amount of training required: One week for this single application. Training is dependant on the type of application. There is some "art or technique" involved.

References: Checked two references (Bayer and Eastman) both said that AIMM has been able to handle all their plugging problems effectively and efficiently. FIU pipe plugging study project manager, Marshall Allen felt the hydrokinetic technology had the best chance of success for the silo application.

Other: Mike Morris and Jennifer Ladd- Lively visited AIMM on June 16, 2004. They observed a demonstration of the process cleaning a heat exchanger. General comment from the demonstration; "The equipment and operation of it are quite simple but very effective."

\section{Photographs:}

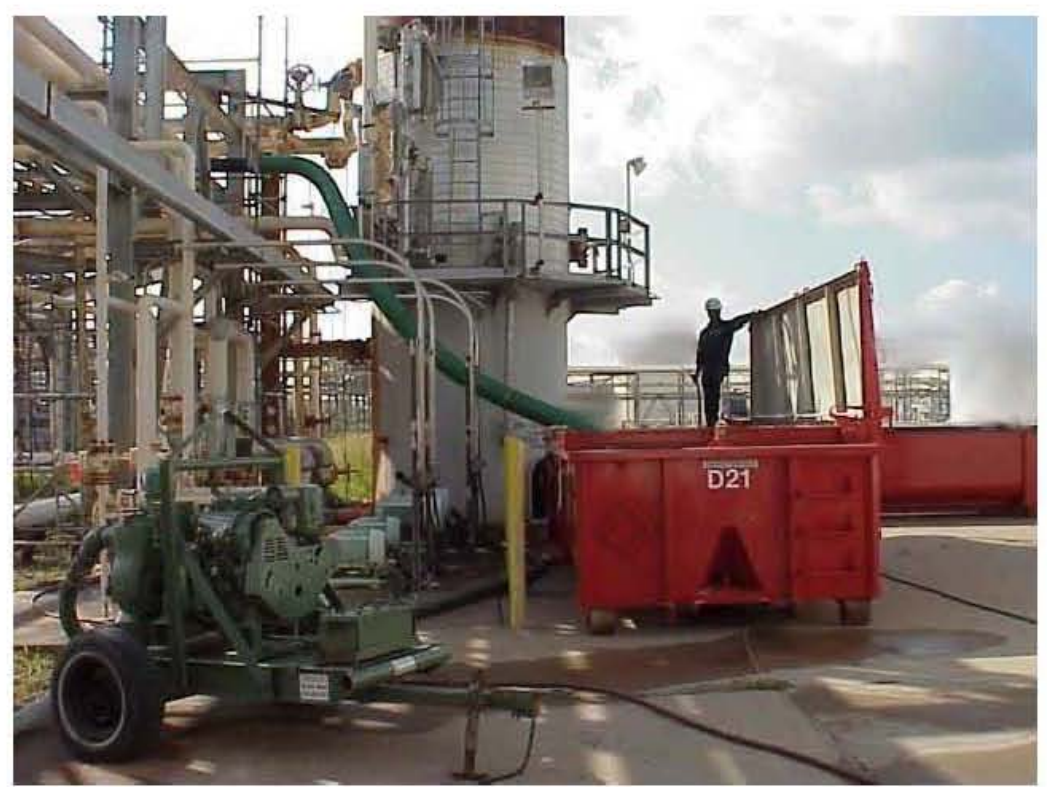

Fig. 9. Typical installation of Hydrokinetic ${ }^{\mathrm{TM}}$ System. 
RPP-RPT-53783, Rev. 0

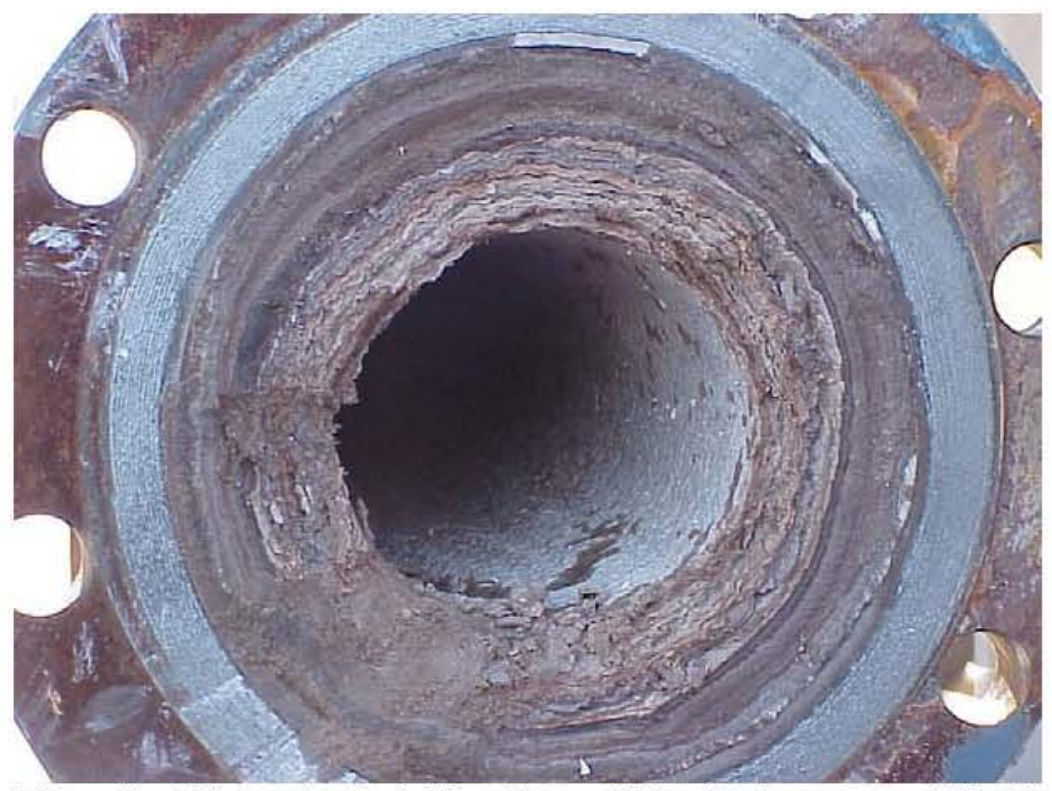

Fig. 10. View of partially plugged line before use of Hydrokinetics ${ }^{\mathrm{TM}}$.

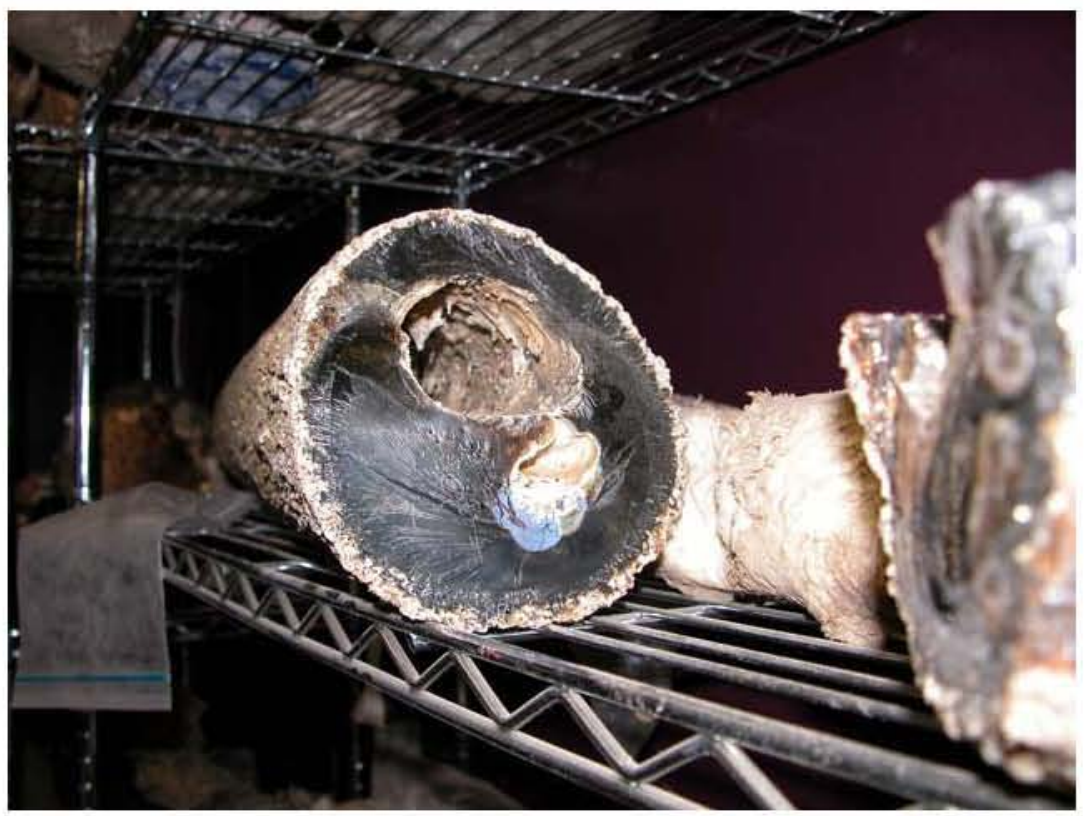

Fig. 11. Solidified line plug removed using Hydrokinetics ${ }^{\mathrm{TM}}$. 


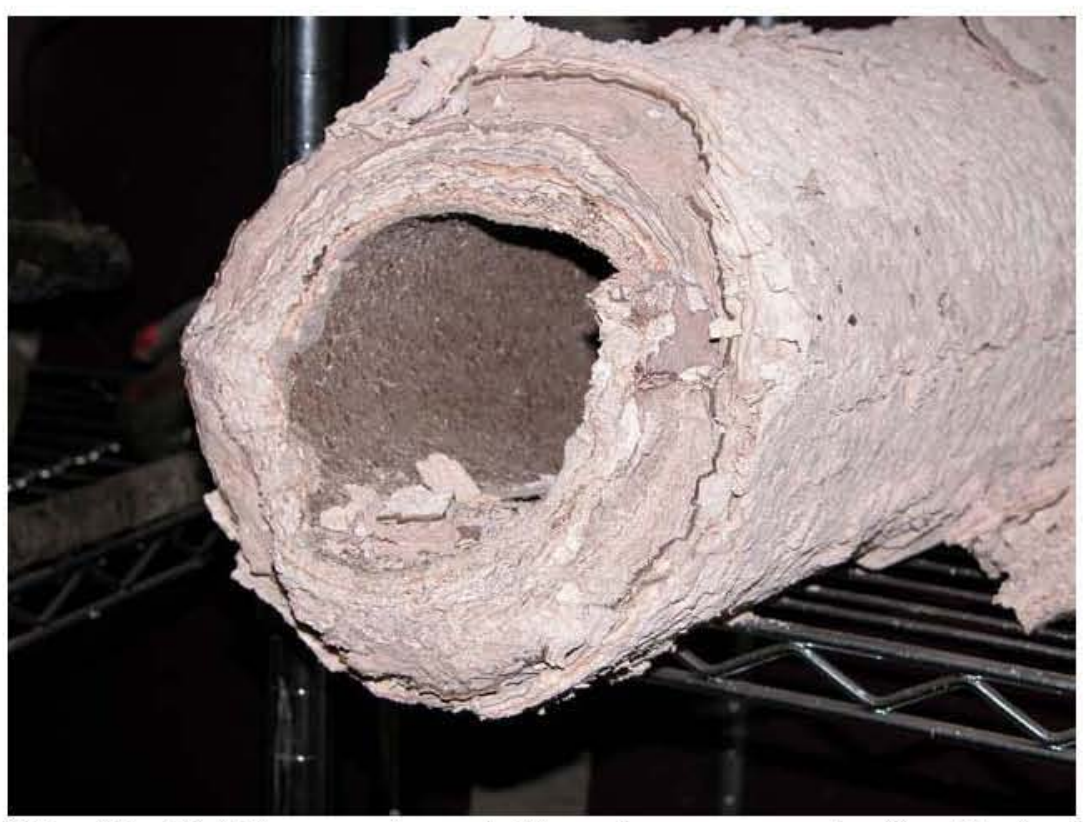

Fig. 12. Calcium carbonate line plug removed using Hydrokinetics ${ }^{\mathrm{TM}}$.

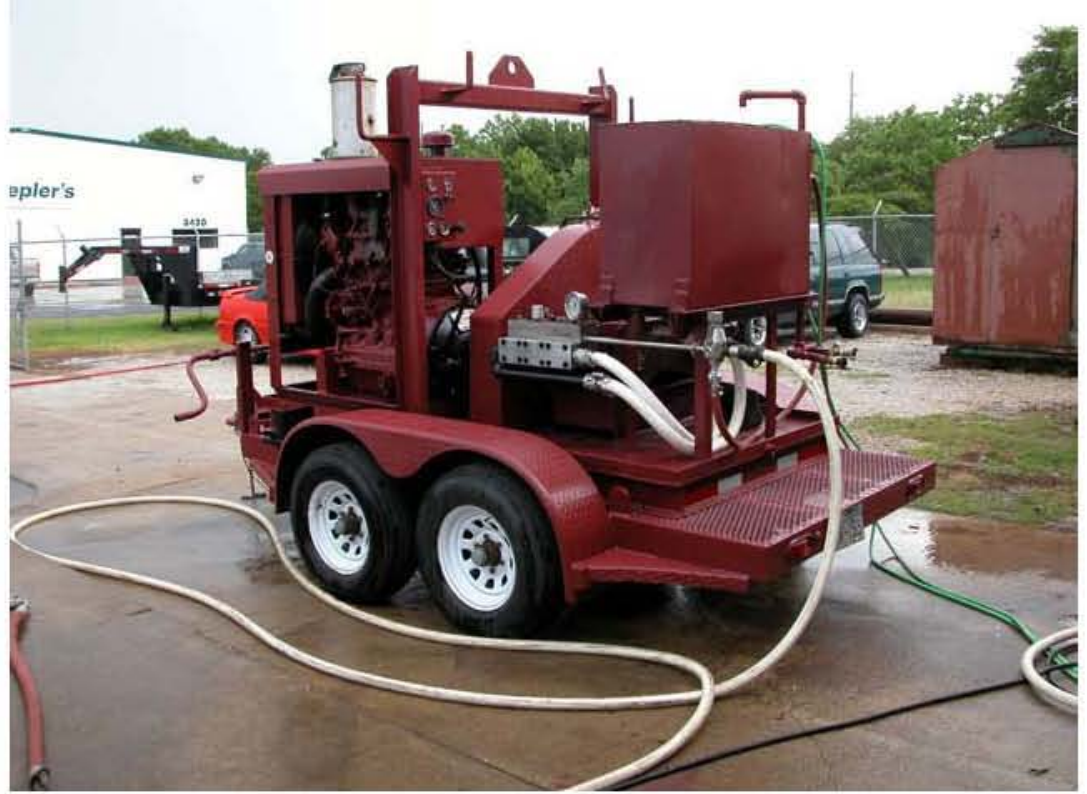

Fig. 13. Trailer mounted high pressure pump. 


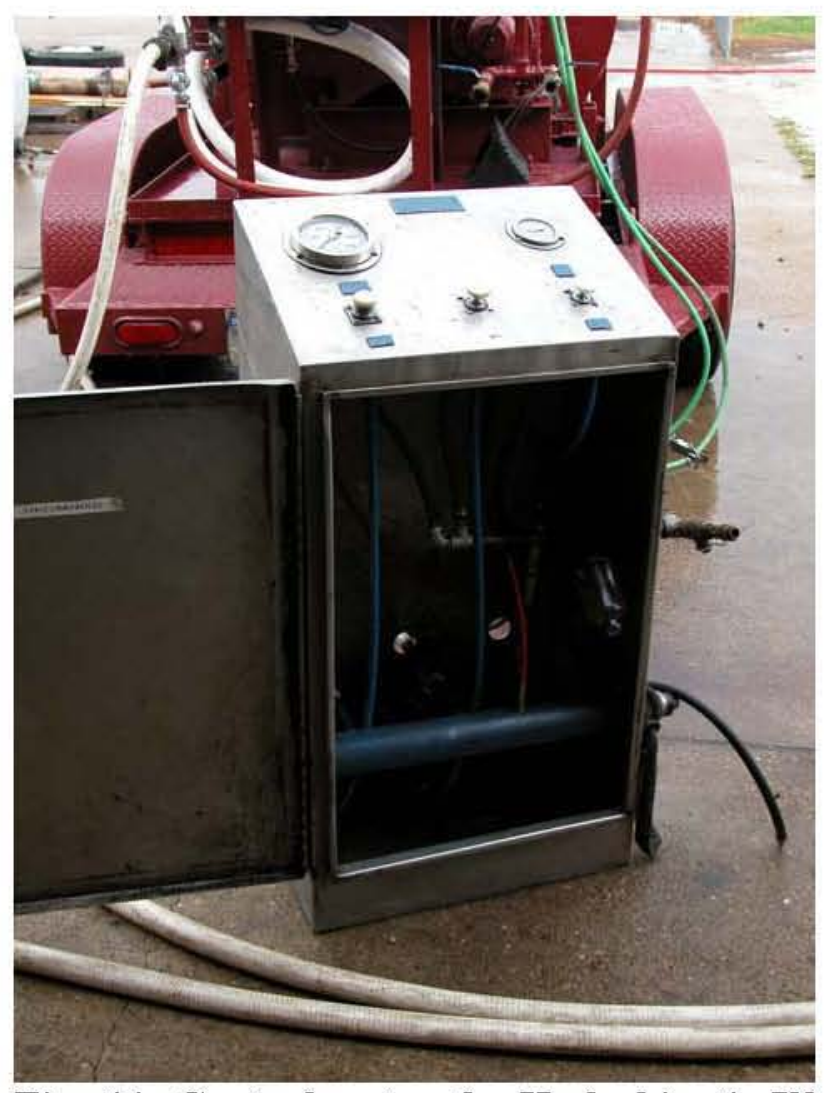

Fig. 14. Control sy stem for Hy drokinetics ${ }^{\mathrm{TM}}$ sy stem.

\section{PARTIAL CLIENT LISTING - REFERENCE LIST}

\begin{tabular}{|c|c|}
\hline $\begin{array}{l}\text { The Atlantic Group } \\
\text { 5426 Robin Hood Rd. } \\
\text { Norfolk, Virginia USA } 23513 \\
\text { Attn: John McLaughlin } \\
\text { Marine Operations } \\
\text { Phone: } 800-446-8131 \\
\text { E-mail: jmclaughlin@atlanticgrp.com }\end{array}$ & $\begin{array}{l}\text { Numerous exchangers aboard ships and } \\
\text { Power Plant Condensers. Fouling material } \\
\text { ranged from totally blocked tubes to those } \\
\text { with marine life and algae. }\end{array}$ \\
\hline $\begin{array}{l}\text { Aqua Drill International } \\
1300 \text { FM } 646 \text { East } \\
\text { Dickinson, TX } 77539 \\
\text { Attn: Chris Geppert } \\
\text { President } \\
\text { Ph: 281-337-0900 } \\
\text { Email: Thomas@aquadrillintemational.com }\end{array}$ & $\begin{array}{l}\text { Various exchangers, pipes, and tank } \\
\text { cleaning applications. Pipe sixes ranged } \\
\text { from } 1 / 2-\text { to } 8 \text {-in. diam with multiple " } 90 \text { 's" } \\
\text { and "T's". }\end{array}$ \\
\hline
\end{tabular}




\begin{tabular}{|c|c|}
\hline $\begin{array}{l}\text { Bayer Chemical } \\
8500 \text { West Bay Road } \\
\text { Baytown, Texas USA } 77520 \\
\text { Attn: Roger Burwell } \\
\text { Maintenance Manager } \\
\text { Phone: } 281-421-0364 \\
\text { E-mail: roger.burwell.b } Q \text { baver.com }\end{array}$ & $\begin{array}{l}\text { Numerous pipe and exchangers plugged } \\
\text { with various types of plastics; HDPE, } \\
\text { LDPE, Makrolon, BPA, etc. Fouling ranges } \\
\text { from very hard to film. }\end{array}$ \\
\hline $\begin{array}{l}\text { Chevron Phillips Chemical Puerto Rico } \\
\text { Core Inc. } \\
\text { Road 710, Bo. fLas Mareas } \\
\text { Guayama, P.R. 00784 } \\
\text { Attn: Carlos E. Pales } \\
\text { Ph: 787-864-1515 x } 2293 \\
\text { Email: palesce } @ \text { cpchem.com }\end{array}$ & $\begin{array}{l}\text { Crude furnace }-8 \text { Pass }-2 \frac{1}{2} \text { in.-diam } \\
\text { tubes. All passes were } 100 \% \text { blocked. }\end{array}$ \\
\hline $\begin{array}{l}\text { Chevron Phillips Chemical Puerto Rico } \\
\text { Core Inc. } \\
\text { Road 710, Bo. fLas Mareas } \\
\text { Guayama, P.R. 00784 } \\
\text { Attn: Hector Marin } \\
\text { Ph: } 787-864-1515 \text { x } 2293 \\
\text { Email: marinha@cpchem.com }\end{array}$ & Multiple pipes. \\
\hline $\begin{array}{l}\text { Chevron Phillips } \\
\text { Bartlesville, Oklahoma USA } \\
\text { Attn: Nathan Stacy } \\
\text { Senior Research Engineer } \\
\text { Phone: 918-661-9596 } \\
\text { E-mail: nestacy@ppco.com }\end{array}$ & $\begin{array}{l}\text { Pipe cleaning of } 1.5 \text { mile } 8 \text {-in. diam. line } \\
\text { blocked with Styrene. }\end{array}$ \\
\hline $\begin{array}{l}\text { ConocoPhillips Alaska, Inc. } \\
\text { Kuparuk Field Planners Office } \\
\text { P.O. Box 196105 } \\
\text { Anchorage, AK 99519-6105 } \\
\text { Attn: Cal Davison / Brett Alexandra } \\
\text { CPF-1 \& CPF-2 Field Planner } \\
\text { Phone: 907-659-7321 } \\
\text { E-mail: n1063@ conocophillips.com }\end{array}$ & $\begin{array}{l}\text { Production crude heaters and coolers. } \\
\text { Crude -water - sand - fouling material. }\end{array}$ \\
\hline
\end{tabular}




\begin{tabular}{|c|c|}
\hline $\begin{array}{l}\text { ConocoPhillips Alaska, Inc. } \\
\text { Kuparuk CPF-1 Unit } \\
\text { P.O. Box 196105 } \\
\text { Anchorage, AK 99519-6105 } \\
\text { Attn: Mark Jerling / Tom Austin } \\
\text { Facility Engineer } \\
\text { Phone: 907-659-7332 } \\
\text { E-mail: N1066@conocophillips.com }\end{array}$ & $\begin{array}{l}3 \text {-in. OD Pipe } 1600 \mathrm{ft} \text { long - oily waste } \\
\text { line. }\end{array}$ \\
\hline $\begin{array}{l}\text { ConocoPhillips Alaska, Inc. } \\
\text { Alpine Operations } \\
\text { P.O. } 196860 \\
\text { Anchorage, AK 99519-6860 } \\
\text { Attn: David Earl } \\
\text { Senior Facility Engineer } \\
\text { Phone: 907-670-4039 } \\
\text { E-mail: alp1201@,conocophillips.com }\end{array}$ & $\begin{array}{l}\text { Production crude coolers ( } 2000 \text { tubes) and } \\
\text { various short sections of pipes from crude } \\
\text { production. }\end{array}$ \\
\hline $\begin{array}{l}\text { DOW Chemical USA } \\
2301 \text { North Brazoport Blvd. } \\
\text { Freeport, Texas USA } 77541 \\
\text { Attn: Jack Russell } \\
\text { Process Cleaning Subject Matter Expert } \\
\text { Maintenance Technical Services } \\
\text { Phone: } 979-238-2382 \\
\end{array}$ & Expert on cleaning process. \\
\hline $\begin{array}{l}\text { DOW Chemical USA } \\
\text { 2301 North Brazoport Blvd. } \\
\text { Freeport, Texas USA 77541 } \\
\text { Attn: Johnny Sweatt } \\
\text { Maintenance Planner/Scheduler } \\
\text { Phone: 979-238-9906 } \\
\text { E-mail: irsweat@dow.com }\end{array}$ & $\begin{array}{l}\text { Perform various heat exchanger and pipe } \\
\text { cleaning jobs in } 3 \text { different units - blocks at } \\
\text { Dow. Large diameter heater - tube } 12 \text {-in. } \\
\text { diam and } 200 \mathrm{ft} \text { long }\end{array}$ \\
\hline $\begin{array}{l}\text { DOW Chemical USA } \\
\text { P.O. Box 150, B1dg. } 807 \\
\text { Plaquemine, Louisiana } 70765 \\
\text { Attn: Ray LeJeune } \\
\text { Process Technologist / Polyethylene A } \\
\text { Phone: } 225-353-4005 \\
\text { E-mail: rtlejeune@dow.com }\end{array}$ & $\begin{array}{l}\text { Various pipe (2- to } 10 \text {-in. diam) plugged } \\
\text { with polyethylene. }\end{array}$ \\
\hline
\end{tabular}




\begin{tabular}{|c|c|}
\hline $\begin{array}{l}\text { DOW Chemical } \\
\text { PO Box 50 } \\
\text { Hahnville, LA 77005 } \\
\text { Attn: Brad Hatfield } \\
\text { Maintenance Engineer } \\
\text { Phone: } 985-783-3897 \\
\text { E-mail: bahatfield@dow.com }\end{array}$ & $\begin{array}{l}\text { Removed approximately } 40-\mathrm{ft} \text { long plug of } \\
\text { polymer from process piping. Cleaned } 3 \text { - } \\
\text { in. diam line from tank farm to reactor } \\
\text { approximately } 1500 \mathrm{ft} \text {. }\end{array}$ \\
\hline $\begin{array}{l}\text { DuPont } \\
\text { DuPont Packing and Industrial Polymers } \\
\text { Sabine River Works } \\
\text { FM } 1006 \\
\text { P.O. Box } 1089 \\
\text { Orange, Texas USA } 77630 \\
\text { Attn: Dan Lynd } \\
\text { Contract Admin } \\
\text { Phone: } 409-886-6106\end{array}$ & $\begin{array}{l}\text { Wax structure coolers, hypersuction coolers, } \\
\text { suction coolers, and recycle discharge } \\
\text { coolers, assorted piping, and reactor tails } \\
\text { line. }\end{array}$ \\
\hline $\begin{array}{l}\text { DuPont } \\
\text { DuPont Packing and Industrial Polymers } \\
\text { Sabine River Works } \\
\text { FM } 1006 \\
\text { P.O. Box } 1089 \\
\text { Orange, Texas USA } 77630 \\
\text { Attn: Natalie Hayes } \\
\text { Division Engineer } \\
\text { Email: natalie.g.hayes@usa.dupont.com }\end{array}$ & \\
\hline $\begin{array}{l}\text { Equate Petrochemicals } \\
\text { Ahmadi (Kuwait City), Kuwait } \\
\text { Attn: Qassem Deshti } \\
\text { Maintenance - Reliability Engineer } \\
\text { PO Box } 9717 \\
\text { Ahmadi } 61008, \text { Kuwait } \\
\text { Tel: 965-326-0326 } \\
\text { Email: qassemdi@shb.equate.com }\end{array}$ & Cleaning of Cycle Gas Cooler \\
\hline $\begin{array}{l}\text { EquiStar Chemical } \\
\text { Channelview, Texas USA } \\
\text { Attn: John Swanson } \\
\text { OP1 Unit Superintendent } \\
\text { Phone: } 281-452-8888\end{array}$ & 2.3 Miles of 4-in. diam line. \\
\hline
\end{tabular}




\begin{tabular}{|c|c|}
\hline $\begin{array}{l}\text { EXXONMobil Plastics } \\
\text { P.O. Box } 1607 \\
\text { Baton Rouge, Louisiana USA } \\
\text { Attn: Richard Bowman } \\
\text { Maintenance Superintendent } \\
\text { Phone: } 225-977-6255 \\
\end{array}$ & $\begin{array}{l}\text { Various high and low pressure piping of } \\
1.0-\text { to } 6 \text {-in.diam and Serpentine coolers. }\end{array}$ \\
\hline $\begin{array}{l}\text { EXXONMobil Chemical } \\
\text { P.O. Box } 241 \\
\text { Baton Rouge, Louisiana USA } 70821-0241 \\
\text { Attn: Kent Allain } \\
\text { Mechanical Supervisor } \\
\text { Phone: } 225-977-8357 \\
\end{array}$ & $\begin{array}{l}\text { Exchanger plugged with cobalt plated } \\
\text { catalyst. }\end{array}$ \\
\hline $\begin{array}{l}\text { EXXONMobil } \\
3700 \text { West } 190^{\text {th }} \text { Street } \\
\text { Torrance, California 90509-2929 } \\
\text { Attn: John Turner } \\
\text { Maintenance Special Projects Planner } \\
\text { Phone: 310-212-2897 } \\
\text { Email: John.w.turner@exxonmibil.com }\end{array}$ & \\
\hline $\begin{array}{l}\text { EXXONMobil } \\
3700 \text { West } 190^{\text {th }} \text { Street } \\
\text { Torrance, California 90509-2929 } \\
\text { Attn: W.G. (Bill) Blashford } \\
\text { Turnaround Planner } \\
\text { Phone: } 310-212-4422 \\
\text { Email: William.g.blashford@exxonmobil.com }\end{array}$ & \\
\hline $\begin{array}{l}\text { EXXONMobil } \\
3700190^{\text {th }} \text { Street, } 102 \text { Hinze Building } \\
\text { Torrance, CA } 90509 \\
\text { Attn: Evan Hyde } \\
\text { Advanced Engineer } \\
\text { Phone: } 310-212-1905 \\
\text { Email: Evan.p.hyde@exxonmobil.com }\end{array}$ & \\
\hline $\begin{array}{l}\text { Kellogg Brown \& Root } \\
8500 \text { West Bay Road } \\
\text { Baytown, Texas USA } 77520 \\
\text { Attn: Roy Weesner } \\
\text { Maintenance Superintended } \\
\text { Phone: 281-383-6448 } \\
\text { E-mail: roy.weesner.b@baver.com }\end{array}$ & $\begin{array}{l}\text { Exchangers and piping fouled with } \\
\text { polypropylene and misc. plastics. } \\
\text { Exchangers } 3 / 4 \text {-in. diam, reboilers, Fin-Fans, } \\
\text { and piping 1- to } 8 \text {-in diam. }\end{array}$ \\
\hline
\end{tabular}




\begin{tabular}{|c|c|}
\hline $\begin{array}{l}\text { Methanex Limited Chile } \\
\text { Cape Horn Facility } \\
\text { P.O. Box 64D } \\
\text { Punta Arenas, Chile } \\
\text { Attn: Pedro Salas } \\
\text { Chief Maintenance Engineer } \\
\text { Phone: 56-61-202230 } \\
\text { Email: psalas@methanex.com } \\
\end{array}$ & Multiple re-boiler cleaning projects. \\
\hline $\begin{array}{l}\text { Noltex } \\
12220 \text { Strange Road } \\
\text { LaPorte, Texas USA } 77572 \\
\text { Attn: Randy Boeding } \\
\text { Plant Manager } \\
\text { Phone: 281-842-5057 } \\
\text { E-mail: randy.boeding@noltex.com }\end{array}$ & $\begin{array}{l}\text { Assorted piping } 1 / 2 \text { - thru } 8 \text {-in. diam } \\
\text { throughout the entire plant. }\end{array}$ \\
\hline $\begin{array}{l}\text { North Atlantic Refining Limited } \\
\text { P.O. Box } 40 \\
\text { Come By Chance, Newfoundland Canada A0B } \\
\text { 1N0 } \\
\text { Attn: Nola Chaytor } \\
\text { Facility Engineer } \\
\text { Phone: 709-463-3484 } \\
\text { E-mail: nolachaytor@na-refining.nf.ca }\end{array}$ & 18 Banks of fin-fans and reboilers. \\
\hline $\begin{array}{l}\text { Shell Chemical Company (Bassell) } \\
473 \text { Hwy } 3142 \\
\text { Taft, Louisiana USA } 70057 \\
\text { Attn: Chad Weidert } \\
\text { Make Change Coordinator } \\
\text { Phone: 504-465-5232 } \\
\text { E-mail: CW315867@msxscc.shell.com }\end{array}$ & $\begin{array}{l}180 \text {-ft U-bundle plugged with tar like } \\
\text { substance. }\end{array}$ \\
\hline $\begin{array}{l}\text { Shell Chemicals America } \\
\text { P.O. Box } 100 \\
\text { Deer Park, Texas USA } 77536 \\
\text { Attn: Christy Duncan } \\
\text { Plant Engineer } \\
\text { Phone: } 713-246-4351 \\
\text { E-mail: cbduncan@shellus.com }\end{array}$ & 4-in. diam crude furnace. \\
\hline
\end{tabular}




\begin{tabular}{|c|c|}
\hline $\begin{array}{l}\text { Shell Gabon } \\
\text { B.P. } 146 \\
\text { Port Gentil Republique Gabonaise } \\
\text { Attn: Edwin Blom } \\
\text { Head of Maintenance OMS/4 } \\
\text { Phone: } 241-55-8502 \\
\text { E-mail: edwin.e.blom } @ \text { shellgb.shell.com }\end{array}$ & $\begin{array}{l}\text { Production crude heat exchangers plugged } \\
\text { tubes from oil productions; crude, water, } \\
\text { sand, wax. }\end{array}$ \\
\hline $\begin{array}{l}\text { Shell Global Solutions International B.V. } \\
\text { Fluid Flow and Flow Assurance } \\
\text { Badhuisweg } 31031 \mathrm{CM} \\
\text { PO Box } 380001030 \mathrm{BN} \\
\text { Amsterdam, The Netherlands } \\
\text { Attn: Jeroen LMM Oomen } \\
\text { Tel: +31 (0) } 206302117 \text { - Mobil: }+31(0) 65512 \\
3394 \\
\text { Email: jeroen.oomen@shell.com }\end{array}$ & Process cleaning experts. \\
\hline $\begin{array}{l}\text { Solvay Polymers } \\
\text { P.O. Box } 1000 \\
\text { Deer Park, Texas 77536-1000 } \\
\text { Attn: John MacDonald } \\
\text { Maintenance Planner } \\
\text { Phone: 713-307-3907 } \\
\text { E-mail: john.macdonald@ solvay.com }\end{array}$ & $\begin{array}{l}\text { Perform various heat exchanger and pipe } \\
\text { cleaning. }\end{array}$ \\
\hline $\begin{array}{l}\text { Texas Eastman } \\
\text { P.O. Box } 7444 \\
\text { Longview, Texas USA } 75607 \\
\text { Attn: Steve Lewis } \\
\text { Phone: } 903-237-5757\end{array}$ & $\begin{array}{l}\text { Various lines including double piped } \\
\text { serpentine cooler, various exchangers. }\end{array}$ \\
\hline $\begin{array}{l}\text { Sterling Chemicals, Inc. } \\
\text { PO Box } 1311 \\
\text { Texas City, TX 77592-1311 } \\
\text { Attn: Lloyd H. Johnson } \\
\text { Maintenance Team Leader - Styrene } \\
\text { Phone: } 409-942-3346 \\
\text { E-mail: liohnson } @ \text { sterlingchemicals.com }\end{array}$ & $\begin{array}{l}80 \text { Banks of Fin-fans and assorted 2- thru } 4- \\
\text { in. diam piping. }\end{array}$ \\
\hline
\end{tabular}


Sterling Chemicals, Inc.

PO Box 1311

Texas City, TX 77592-1311

Attn: Tommy Baker

Maintenance Planner

Phone: 409-942-3346

E-mail: tlbaker@sterlingchemicals.com
Various piping to remove sea water fouling and misc. styrene lines. 


\title{
Completed Pipeline Unplugging Vendor Survey Form for Fluidic Wave Action Technology Provider AEA Technologies
}

\author{
Company Contact Information \\ AEA Technologies \\ 184 B Rolling Hill Rd \\ Mooresville, North Carolina 28117 \\ Phone: 704-799-2707 \\ Fax: 704-799-6426 \\ Email: paul.murray@aeatech.com \\ Contact: Paul Murray \\ Process name: Fluidic Wave Action
}

Process description: AEA Technologies' unplugging process is based on a fluid waveaction principle that operates much like ocean wave-action on beach erosion coupled with positive and negative pressure pulses that tend to loosen the blockage. The cleaning process can be aided by use of a solvent in lieu of water. It can operate on a long pipeline that has drained down below a blockage. The system consists of a water/solvent tank, pressurized/vacuum vessel, portable air compressor, jet pump pairs and valve manifold, fluidic control unit, vacuum finishing pump, system controller, and system module. A vacuum pump is used to evacuate any air that may be present in the pipeline below the blockage in elevation. Once a vacuum has been established, a ball valve is opened, and water or other solvent is allowed to back-fill the pipeline. The fluidic control system is then used to provide pressure and vacuum to the fluid in a cyclic manner. During the drive cycle, fluid impacts the blockage as a wave flowing under the air bubble at the high point of the blockage, and during the suction cycle, water retreats away from the blockage. These cycles are repeated many times until the blockage is eroded away. The frequency and duration, as well as the pressure, of each cycle can be controlled via the fluidic control unit. This, coupled with the dissolving action of a selected solvent (if needed) and the physical action of the vacuum and pressure cycles, works to both erode and loosen the blockage. 

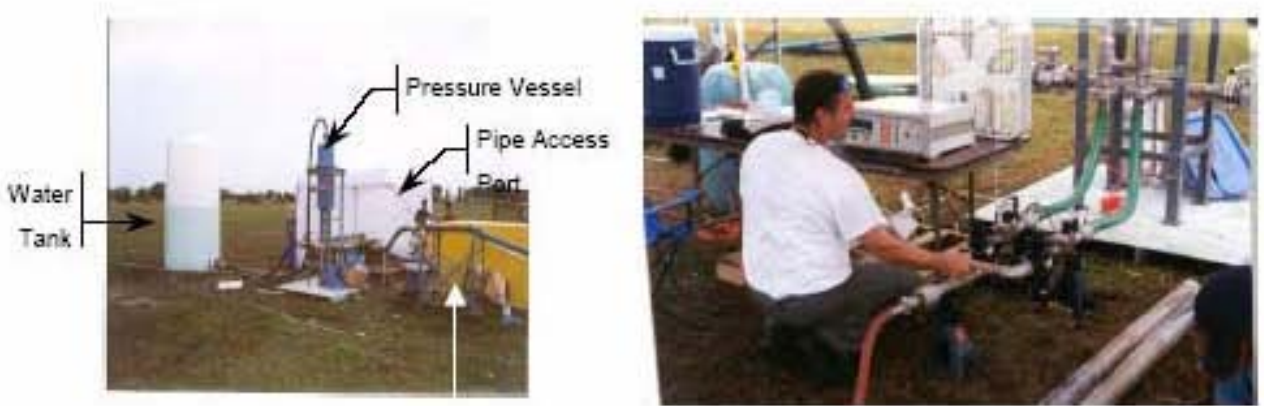

Fig. 15. Equipment and control module used in the FIU dem onstration of the AEA Technology Fluidic Wave Action process. Source: M.A. Ebadian, Plugging Prevention and Unplugging of Waste Transfer Pipelines, Part 1-Equipment Tests of Blockage Locating, Detecting, and Unplugging Technologies on the Full-Size Test Beds, HCET1998-M004-001-04, Hemispherical Center for Environmental Technology, Florida International University, 2002.

\section{Advantages:}

1. Unaffected by piping configuration or distance from plug.

2. Can be operated remotely, minimal exposure.

3. Typically requires no system modification; hook up to single access point connection.

4. Operating equipment minimal consisting of vacuum pump, air injection and water injection system.

5. Operation simple

6. Quick and easy mobilization and demobilization.

7. Low pressure.

\section{Disadvantages:}

1. Process line fluid does come in contact with equipment.

2. Technology has been demonstrated (FIU) for line unplugging but has not been used in an actual application.

3. Not a lot of data available to predict duration for various types of plugging materials.

Company safety record: 100,000 thours of operation at 54 locations. Process for line unplugging has no safety record. However this same process is also used for tank cleanout and has operated at many DOE sites including ORNL, Mound, LANL and INEL.

Failure rate and reasons for failures: No record for pipe unplugging.

Company size (employees, sales): 40,000 employees' world wide with $\sim 50$ employees in USA. 
Industries served: Nuclear

Comparison with competing technologies: Hydrokinetics, hydroblasting, chemical cleaning, mechanical cleaning

Current projects: Ongoing services to the nuclear industry and DOE.

Experience working with DOE? Sites and Projects: Many sites including Fernald.

Radioactive environment experience: Yes

Experience with various lengths, diameters, and materials of piping: Based on the FIU testing $\sim 1,700$ linear feet and various diameters. .

Experience with plugs of various materials (i.e. $\mathrm{CaCO}_{3}$ ): minimal

Maximum size of plugs cleared and time required: See above.

Experience with closed systems and/or remote operations: System is effective in this environment. However the process fluid does come in contact with the unplugging equipment.

Contracting services: Purchase only. There is a system at Mound that could be refurbished for $\sim \$ 100 \mathrm{~K}$. New equipment would cost $\$ 250-300 \mathrm{~K}$ Training and consulting $\operatorname{cost} \sim \$ 1,500 /$ day.

System connections needed (size and type): Single flange - Variable size and type.

Number of people required to operate: 2

Amount of training required: 1-2 days

Other: Mike Morris and Jennifer Ladd-Lively meet with Paul Murray and T. J. Abraham of AEAT. AEAT has a project at ORNL for tank cleanout and we will be visiting the site on Monday. 
RPP-RPT-53783, Rev. 0

Appendix E

PIPE-PULSE PATENT 
(12) INTERNATIONAL APPLICATION PUBLISHED UNDER THE PATENT COOPERATION TREATY (PCT)

(19) World Intellectual Property Organization International Bureau

(43) International Publication Date 31 March 2011 (31.03.2011)

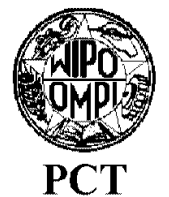

||||||||||||||||||||||||||||||||||||||||||||||||||||||||||||||||||||||||||||||||||||||||||

(10) International Publication Number WO 2011/036502 A1
(51) International Patent Classification: B08B 9/032 (2006.01)

(21) International Application Number:

$\mathrm{PCT} / \mathrm{GB} 2010 / 051623$

(22) International Filing Date; 28 September $2010(28.09 .2010)$

(25) Filing I anguage:

(26) Publication Language:

Finglish

(30) Priority Data

0916887.328 September 2009 (28.09.2009) (il3

(71) Applicant (for all designated States except US): PARADIGM FLOW SOLUTIONS LIMITED [ /GB]; 7 Queens Terrace, Aberdeen AB10 1XL (GB).

(72) Inventor; and

(75) Inventor/Applicant (for $U S$ only): MACKENZIE, Hugh [GB/GB]; c/o Paradigm Flow Solutions Limited, 7 Queens Terrace, Aberdeen AB10 1XL (GB).

(74) Agent: LINCOLN IP; 9 Victoria Street, $\Lambda$ berdeen, $\Lambda$ b erdeenshire $\mathrm{AB} 10 \mathrm{1XB}(\mathrm{GB})$.

(81) Designated States (unless otherwise indicated, for every kind of national protection available): $\mathrm{AE}, \mathrm{AG}, \mathrm{AL}, \mathrm{AM}$, $\mathrm{AO}, \mathrm{AT}, \mathrm{AU}, \mathrm{AZ}, \mathrm{BA}, \mathrm{BB}, \mathrm{BG}, \mathrm{BH}, \mathrm{BR}, \mathrm{BW}, \mathrm{BY}, \mathrm{BZ}$ $\mathrm{CA}, \mathrm{CH}, \mathrm{CL}, \mathrm{CN}, \mathrm{CO}, \mathrm{CR}, \mathrm{CU}, \mathrm{CZ}, \mathrm{DE}, \mathrm{DK}, \mathrm{DM}, \mathrm{DO}$,
DZ, EC, EE, EG, ES, FI, GB, GD, GE, GH, GM, GT, $\mathrm{HN}, \mathrm{HR}, \mathrm{HU}, \mathrm{ID}, \mathrm{IL}$, IN, IS, JP, KL, KG, KM, KN, KP, KR, KZ, LA, LC, LK, LR, LS, LT, LU, LY, MA, MD, $\mathrm{ME}, \mathrm{MG}, \mathrm{MK}, \mathrm{MN}, \mathrm{MW}, \mathrm{MX}, \mathrm{MY}, \mathrm{MZ}, \mathrm{NA}, \mathrm{NG}, \mathrm{NI}$, NO, N', OM, PE, PG, PH, PL, P'T, RO, RS, RU, SC, SU, SE, SG, SK, SL, SM, ST, SV, SY, TH, TJ, TM, TN, TR, TT, TZ, UA, UG, US, UZ, VC, VN, ZA, ZM, ZW.

(84) Designated States (unless atherwise indicated, for every kind of regional protection available): ARIPO (BW, $\mathrm{GH}_{3}$ GM, KE, LR, LS, MW, MZ, NA, SD, SL, SZ, TZ, UG, ZM, ZW), Eurasian (AM, AZ, BY, KG, KZ, MD, RU, TJ, TM), European (AL, AT, BE, BG, CH, CY, CZ, DE, DK, EE, ES, FI, FR, GB, GR, HR, HU, IE, IS, IT, LT, LU, LV, MC, MK, MT, NL, NO, PL, PT, RO, SE, SI, SK, $\mathrm{SM}, \mathrm{TR}), \mathrm{OAPI}(\mathrm{BF}, \mathrm{BJ}, \mathrm{CF}, \mathrm{CG}, \mathrm{CI}, \mathrm{CM}, \mathrm{GA}, \mathrm{GN}, \mathrm{GQ}$, GW, ML, MR, NE, SN, TD, T(r).

Declarations under Rule 4.17:

- as to the applicant's entillement to claim the priority of the earlier application (Rule 4.17(iii))

Published:

- with international search report (Art. 21(3))

- before the expiration of the time limit for amending the claims and to be republished in the event of receipt of amendments (Rule 48.2(h))

(54) Title; IMPROVED BLOCKAGE REMOVAL APPARATUS AND METHOD

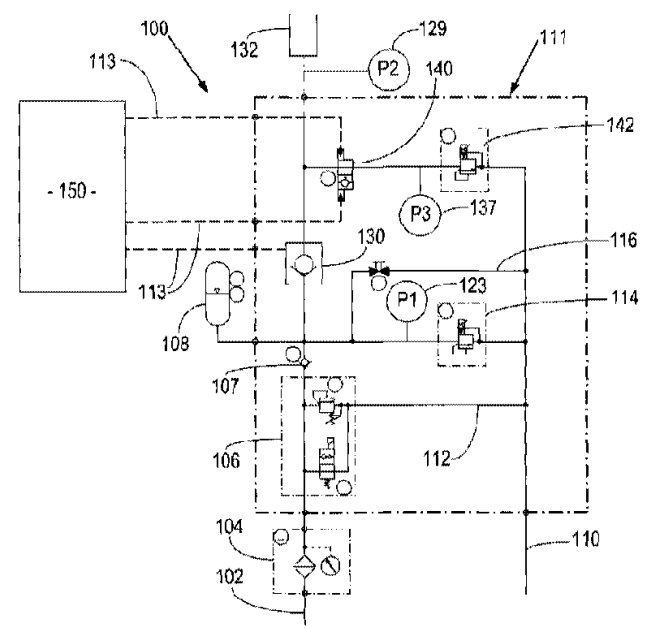

Fig. 2

(57) Abstract: The invention provides a method and apparatus for removing a blockage from a fluid conduit. An apparatus comprises a tirst portion containing a fluid volume separated from the fluid conduit via a controllable valve. The valve is cyclically opened and closed such that a pressure differential between the first portion and the fluid conduit causes a scries of pressure pulses in the fluid conduit. The pressure differential is regulated to control the amplitude of the pressure pulses of the series. 
$1 \quad$ Improved blockage removal apparatus and method

3 The present invention relates to an apparatus and method for cleaning of fluid conduits or

4 vessels. The invention has particular application to the removal of blockages from fluid

5 conduits used in the hydrocarbon exploration and production industry, for example fluid

6 conduits contained within umbilicals. The invention also relates to a method and

7 apparatus for generating a pulse in a fluid conduit or vessel.

8

9 Background to the Invention

11 During hydrocarbon exploration and production processes, it is common for the interiors of 12 fluid conduits, including pipelines, wellbores, risers and umbilicals to become fouled. The

13 fouling often leads to the formation of a blockage within the fluid conduit, which may be as

14 a result of a gradual build-up of material on the inside surface of the conduit or the

15 formation of a plug as an unwanted by-product of a (possibly unanticipated) chemical

16 reaction. The blockage prevents further use of the fluid conduit and must be removed

17 before the process can continue. 
1
A range of techniques have been developed for removing blockages from fluid conduits. These range from lance or nozzle jet systems, which are inherently limited in their range, and ultrasonic systems which apply acoustic energy to the fluid to attempt to induce cavitation in the fluid.

It has also been proposed to use pulses of pressurised fluid in order to remove material from internal surfaces of fluid conduits and vessels. US $5,183,513$ describes a system in which a high pressure pump is coupled to a fluid vessel via a pressure regulator. A controllable valve is located in the fluid line between the vessel and the pressure regulator, and is connected to the vessel via a controllable valve. The valve is cyclically opened and closed to allow pressure pulses to pass into the vessel. The operation of the valve is controlled such that the pulses are formed at frequencies, pressures and temperatures that induce cavitation within the fluid which is said to remove material from the internal surfaces of the vessel.

Cavitation is undesirable in many applications because the implosion of bubbles can pit or damage the internal surfaces of a fluid system.

Pressure pulse systems such as those described in US 5,183,518 are deficient in controlling the magnitude of the pulses. This presents a particular difficulty when the fluid conduit or vessel is sensitive to pressure, as may be the case in many hydrocarbon production and transportation installations. There is a concern amongst operators of such installations that uncontrolled pulses which are allowed to pass into a fluid system will cause damage resulting in reduced integrity and a shortened operating lifetime.

There is therefore a need for a method and apparatus for cleaning pipeline systems which is improved with respect to the previously proposed systems.

It is amongst the aims and objects of the invention to provide a method and apparatus for cleaning of fluid conduits or vessels which allows the delivery of fluid pressure pulses with controlled pressure pulses.

Further aims and objects will become apparent from reading the following description. 


\footnotetext{
1 Summary of the Invention

2

3 According to a first aspect of the invention there is provided a method for removing a

4 blockage from a fluid conduit, the method comprising:

5 providing an apparatus comprising a first portion containing a fluid volume separated from

6 the fluid conduit via a controllable valve;

7 cyclically opening and closing the controllable valve such that a pressure differential

8 between the first portion and the fluid conduit causes a series of pressure pulses in the

9 fluid conduit;

10 regulating the pressure differential to control the amplitude of the pressure pulses of the 11 series.

13 The method may comprise regulating the pressure of the fluid volume in the first portion so

14 that it is greater than the pressure in the fluid conduit (referred to as a positive pressure

15 differential); and

16 transmitting positive pressure pulses to the conduit.

18 Alternatively the method may comprise regulating the pressure of the fluid volume in the

19 first portion so that it is less than the pressure in the fluid conduit (referred to as a negative

20 pressure differential); and transmitting negative pressure pulses to the conduit.

The method may comprise transmitting both positive and negative pressure pulses into the

23 fluid conduit. For example, the method may comprise transmitting a series of positive

24 pressure pulses into the system (during a pressuring up cycle) followed by a series of

25 negative pressure pulses (during a pressure bleeding cycle) or vice versa.

In the prior art systems, allowing pressure pulses to be transmitted to a fluid conduit changes the fluid pressure in the conduit. Where positive pressure pulses are transmitted the fluid pressure in the conduit is increased with every pulse, thereby reducing the differential pressure and the magnitude of subsequent pulses. Where negative pressure pulses are transmitted, a gradual equalisation of pressure may occur (in a closed system) which reduces the magnitude of subsequent pulses. Alternatively, for a system in which

33 the first portion is held at low pressure, the magnitude of the negative pulses transmitted may be undesirably large. 
1 The method allows the pressure regulator to compensate for pressure changes in the

2 system to maintain the pressure differential within an acceptable and preferred range.

3 This allows control of the amplitude of the pressure pulses generated in the fluid conduit.

4 The method may therefore comprise a feedback mechanism which monitors a change to

5 the pressure conditions due to the transmission of a pulse and adjusts or regulates a

6 pressure differential in response.

8 Preferably the method includes measuring (a second) fluid pressure in the fluid conduit.

9 The method may include measuring an average pressure in the fluid conduit, for example 10 over a period of at least one pulse cycle.

The method may include the step of measuring a first fluid pressure in the first portion.

13 The pressure differential may then be calculated from the first and second fluid pressures.

14 Alternatively the first fluid pressure may be determined indirectly from parameters and/or calibration of a pressure regulator used to regulate the pressure in the first portion.

Preferably the first and/or second fluid pressure measurements are communicated to a

18 control module, which may be in the form of a programmable logic controller (PLC).

19 Preferably the control module controllably operates the valve.

Where there is a pressure bleed cycle from the fluid conduit, the method may comprise the step of directing fluid through a second controllable valve by cyclically opening and closing the valve. The second controllable valve is preferably located on a fluid return line. flow path. This facilitates the use of an advantageous class of valve as will be described below. a blockage from a fluid conduit or vessel, the apparatus comprising:

31 a first portion containing a fluid volume;

32 a connector for coupling the first portion to the fluid conduit or vessel;

33 a controllable valve disposed between the first portion and the connector;

34 at least one pressure sensor for measuring a pressure in the fluid conduit or vessel;

35 a control module for opening and closing the valve; 
and a fluid pressure regulator configured to control the fluid pressure in the first portion in response to a signal from the pressure sensor.

Preferably the apparatus is configured to cyclically open and close the valve to transmit pressure pulses into a fluid conduit to remove a blockage. Preferably the apparatus is

6 configured to measure a differential pressure, which may be a differential pressure across 7 the valve.

9 Preferably the apparatus is arranged to be coupled to a high pressure pump. Alternatively 10 a high pressure pump may form a part of the apparatus.

Preferably the pressure regulator comprises a pressure relief valve, which may be a 13 proportional pressure relief valve. The pressure regulator may therefore be capable of 14 balancing a reduction in the pressure differential across the controllable valve by bleeding 15 pressure from the low pressure side of the controllable valve.

17 The pressure regulator may be a two-way pressure regulator, and more preferably is 18 electronically controllable. The apparatus may comprise a control module for configuring 19 operational parameters of the apparatus. The operational parameters may be one or more 20 selected from the group consisting of: operating frequency; pulse width; maximum 21 differential pressure $(\mathrm{dP})$; maximum pressure; and minimum pressure.

22
The apparatus may comprise a fluid return line from the fluid conduit to the first portion. The fluid return line may comprise a second valve. Preferably the second valve is configured for controllable transmission of fluid pressure pulses, e.g. during a bleed-down cycle.

At least one of the valve and/or the second valve is preferably an oscillating valve, and more preferably is a fast-acting oscillating valve. At least one is may be electronically operable, and in one embodiment is a solenoid-actuated oscillating valve. At least one of the valves may have an orifice in the range of $10 \mathrm{~mm}$ to $20 \mathrm{~mm}$, preferably about $15 \mathrm{~mm}$. At least one of the valves may have a flow rate in the range of 300 to 500 litres per minute, preferably about 400 litres per minute. 
1 At least one of the valve and/or the second valve may be a hydraulically actuated valve.

2 The apparatus may comprise a hydraulic control system for the hydraulically actuated 3 valve.

4

5 Preferred or optional embodiments of the second aspect of the invention may comprise

6 preferred or optional features of the first aspect of the invention or vice versa.

8 According to a third aspect of the invention there is provided a hydrocarbon production or

9 transportation system comprising a fluid conduit and an apparatus for removing a blockage

10 from the fluid conduit coupled to the conduit, the system comprising a first portion

11 containing a first fluid volume;

12 a controllable valve disposed between the first portion and the fluid conduit;

13 a pressure source for providing pressurised fluid to the first portion;

14 a control module configured for opening and closing the valve to allow pressure pulses into

15 the fluid conduit;

16 pressure sensing means for determining a pressure differential across the controllable

17 valve;

18 and a fluid pressure regulator configured to control the fluid pressure in the first portion in

19 response to a signal from the pressure sensing means.

20

21 The system may comprise a dynamic pressure regulator, for example using a closed fluid

22 system using a two-way regulator, or may comprise a static pressure regulator, for

23 example using pressure relief valves.

24

25 Preferred or optional embodiments of the third aspect of the invention may comprise

26 preferred or optional features of the first or second aspects of the invention or vice versa.

27

28 According to a fourth aspect of the invention there is provided an apparatus for removing a blockage from a fluid conduit or vessel, the apparatus comprising:

30 a first portion containing a fluid volume;

31 a connector for coupling the first portion to the fluid conduit or vessel;

32 a first controllable valve disposed between the first portion and the connector configured to

33 transmit positive pressure pulses in a direction from the first portion to the connector;

34 a fluid return line; 
1 a second controllable valve disposed between the first portion and the connector

2 configured to bleed pressure pulses in a direction from the connector to the first portion;

3 and a control module for opening and closing the first and second valves.

4

5 Preferred or optional embodiments of the fourth aspect of the invention may comprise

6 preferred or optional features of the first to third aspects of the invention or vice versa.

8 The invention also extends to the cleaning of the interior surfaces of pipelines, conduits, or

9 vessels and therefore according to further aspects of the invention there are provided a

10 method and apparatus of cleaning the interior surface of fluid systems comprising the

11 features of the first and second aspects of the invention.

12

13 According to a fifth aspect of the invention there is provided an apparatus for generating a

14 pressure pulse in a fluid conduit or vessel, the apparatus comprising:

15 a first portion containing a fluid volume;

16 a connector for coupling the first portion to the fluid conduit or vessel;

17 a controllable valve disposed between the first portion and the connector;

18 at least one pressure sensor for measuring a pressure in the fluid conduit or vessel;

19 a control module for opening and closing the valve;

20 and a fluid pressure regulator configured to control the fluid pressure in the first portion in

21 response to a signal from the pressure sensor.

22

23 Preferred or optional embodiments of the fifth aspect of the invention may comprise

24 preferred or optional features of the first to fourth aspects of the invention or vice versa.

According to a sixth aspect of the invention there is provided an apparatus for generating a pressure pulse in a fluid conduit or vessel, the apparatus comprising:

28 a first portion containing a fluid volume;

29 a connector for coupling the first portion to the fluid conduit or vessel;

30 a first controllable valve disposed between the first portion and the connector configured to

31 transmit positive pressure pulses in a direction from the first portion to the connector;

32 a fluid return line;

33 a second controllable valve disposed between the first portion and the connector

34 configured to bleed pressure pulses in a direction from the connector to the first portion;

35 and a control module for opening and closing the first and second valves. 
1

Preferred or optional embodiments of the sixth aspect of the invention may comprise preferred or optional features of the first to fifth aspects of the invention or vice versa.

\section{Brief Description of the Drawings}

There will now be described, by way of example only, an embodiment of the invention with reference to the drawings, of which:

Figure 1 is a process and instrumentation diagram of a system according to a first embodiment of invention; and

Figure 2 is a process and instrumentation diagram of a system according to a first embodiment of invention.

\section{Detailed Description}

Referring firstly to Figure 1, there is shown generally depicted at 10 a fluid system comprising an apparatus 11 and a fluid conduit 32, which in this case is an umbilical. The fluid conduit 32 is coupled to the apparatus 11 via a suitable interface (not shown) and an isolation valve 30 . The apparatus 11 is also connected to a fluid source 12 via a high pressure pump 14. A particulate filter 16 is located between the pump 14 and a two-way pressure regulator 18 . The two-way pressure regulator 18 of this embodiment is a standard pressure regulator modified so that pressure output can be controlled by a computer or another electronic device. Suitable commercially-available examples include the Automated Pressure Regulators sold by Advanced Pressure Products of Ithaca, New York, United States.

A pressure accumulator 22 is connected to the pressure regulator 18 via a check valve 20 . The accumulator 22 prevents loss of amplitude during the transmission of pulses as will be described below. Line 24 connects the accumulator 22 to a first oscillating valve 26 , which separates a first portion of the apparatus from a line 28 in fluid communication with the conduit 32 . 
The oscillating valve 26 is in this embodiment a solenoid-actuated stem valve which is capable of rapid actuation and opening and closing at high frequencies (for example, up to 10 cycles per second). A suitable valve will have a valve orifice of around $15 \mathrm{~mm}$ and a flow of around 400 litres per minute. It has been found that this class of valve has

5 particular benefits in many blockage removal applications due to its rapid actuation and

6 high flow rate characteristics.

In addition, the fast actuation of the solenoid-actuated valves allows generation of welldefined, repeatable pulses which may be useful in blockage location systems which use transit time to estimate the location of a blockage. A pressure sensor 82 measures the occurrence of a pressure pulse in the conduit, and transmits the measurement data to an external module 80. Transit time between the initial pulse and the pulse reflected from the blockage in the conduit allows calculation of the distance to the blockage.

However, one limitation of some solenoid-actuated valves is that they may not rapidly open and close when exposed to pressure differentials in two directions. For example, valve 26 is only capable of rapidly opening and closing when the pressure differential is in

18 the direction of the arrow; i.e. when the higher pressure is in the line 24. The present

19 embodiment therefore comprises a fluid return line 34 which joins the line 28 between the

20 valve 26 and the fluid conduit 32 . Located in the fluid return line is a second oscillating valve 36 , of the same type as valve 26 , which separates line 38 from line 34 and the connected conduit 32 . The valve 36 is arranged for fast actuation when the higher pressure is in the line 34. This arrangement allows the benefits of the invention to be exploited during both the pressure-up cycle and the pressure-bleed cycle (as described below). Located between the oscillating valve 36 and the line 42 to the pressure regulator 18 is a controllable dump valve 40 .

The apparatus 11 also includes a control unit 50 in the form of a programmable logic controller (PLC) 50. The PLC 50 communicates with the valves 26,36 and 40 , controlling their operation. The PLC 50 also controls the operation of the pressure regulator 18. An external control panel 52 allows the user operation of the PLC 50. The control panel has controls for the operating frequencies of the valve oscillators 26 and 36, the maximum differential pressure $(\mathrm{dP})$, the maximum pressure and the minimum pressure. The control 
panel also has an on/off switch, a pressure regulator override function and visual

4 A power distribution system 60 is provided in the apparatus 11 to receive power from an

5 external power supply 62 and distribute power to the pressure regulator 18 , the valves 26 ,

636 and 40, and the PLC 50.

Pressure sensor 23 measures the pressure $\mathrm{P} 1$ in the first portion of the apparatus between the accumulator 22 and the valve 26 . Similarly, pressure sensor 29 measures the pressure P2 in the line between the valve 26 and the fluid conduit (i.e. the fluid conduit pressure), and pressure sensor 44 measures the pressure $\mathrm{P} 4$ in the line in the return line 42. Each pressure sensor provides a measurement signal to the PLC 50. Optionally an additional pressure sensor 37 is provided to measure the pressure in between the valve 36 and the dump valve 40 and provide a signal to the PLC 50.

Operation of the system 10 will now be described. In an initial configuration the valve oscillators 26 and 36 will normally be closed. The two-way regulator 18 is fully open. The operator enters the settings via the control panel 52 , which include the operating frequencies of the valve oscillators 26 and 36 , the maximum differential pressure $(\mathrm{dP})$, the maximum pressure and the minimum pressure.

To begin unblocking the conduit 32 , the pump 14 is activated to pump fluid from the fluid tank 12 through the apparatus 11 . The oscillator valve 26 remains closed, and pressure sensor $\mathrm{P} 2$ takes a pressure measurement in line 28 (which is open to the conduit 32 ). The PLC 50 reads the pressure signal and adjusts the two way regulator 18 to increase the pressure at $\mathrm{P} 1$ in line 24 to a value within a pre-determined range (for example plus or minus $5 \%$ ) of the preset value of $P 2+d P$. When the value of $P 1$ is reached, the PLC 50 commands the oscillator valve 26 to cyclically open and close at its preset frequency. Positive pressure pulses are therefore transmitted into the conduit 32 to begin to remove the blockage. Transmission of pressure pulses increases the pressure P2. 
operation then valve oscillator 26 is automatically closed. When the pressure P1 comes back within the required range of $\mathrm{P} 2+\mathrm{dP}$ the oscillator valve 26 recommences cycling.

When the pressure $\mathrm{P} 2$ in the fluid conduit reaches the preset maximum, the bleed-down cycle commences. Valve oscillator 26 is held in the open position so that pressure is not trapped in the accumulator 22 and the whole system 10 can be bled down. Valve oscillator 36 is closed, dump valve 40 is opened, and pressure P4 in line 42 is built up by the pressure regulator 18 Optional pressure sensor 37 may read the pressure P3 throughout the pressure build up operation to ensure there has been no bypass.

When pressure P4 in line 42 is adjusted by the pressure regulator 18 to a value within a preset range (for example $10 \%$ below the set value) of $\mathrm{P} 2-\mathrm{dP}$, the valve oscillator 36 is activated to allow pressure to be bled from the fluid conduit 32 in a controlled manner. Negative pressure pulses are therefore transmitted into the conduit 32, which increases the pressure P4 and decreases the pressure P2. During the transmission of pulses, the two-way regulator 18 is automatically adjusted by the PLC 50 to maintain the pressure P4 in line 42 within the required range of $\mathrm{P} 2-\mathrm{dP}$.

If P4 falls outside of a predetermined range (for example $10 \%$ below the set value) of P2 $\mathrm{dP}$ during this operation then valve oscillator 26 is automatically closed. When the pressure $\mathrm{P} 1$ comes back within the required range of $\mathrm{P} 2+\mathrm{dP}$ the oscillator valve 36 recommences cycling.

When the minimum pressure is reached in the fluid conduit 32 , the oscillator valves 26,36 and the dump valve 40 are closed. The two-way regulator 18 increases pressure P1 until it is in within the required range of $\mathrm{P} 2+\mathrm{dP}$ and the process is repeated.

The described embodiment allows the generation of pressure pulses of known amplitude throughout the pressure-up and bleed-down cycles, in contrast to the prior art proposals which do not adequately address the issues of compensating for pressure changes which result from the transmission of pulses. Providing amplitude control allows the parameters of the system to be set closer to the acceptable limits of the fluid conduit, with a higher

33 level of confidence that the conduit 32 will not be damaged. Ultimately this provides a greater range of operating parameters than those available in the prior art. 
The use of solenoid-actuated valves provides the advantages of quick actuation and automated operation. This facilitates operation at high frequencies without reliance on human operators to manually open and close the valves. The choice of valves has the additional benefit of producing well-defined, repeatable pulses which may be detected in or near the fluid conduit to locate the blockage. In certain applications, it may be desirable to use an alternative system configuration with different valve, actuation, and/or pressure regulation components. Figure 2 is an example of a system which is particularly suited for use with larger bore pipeline systems (for example inner diameters in the range of around 4 to 10 inches (about 100 to $250 \mathrm{~mm}$ )), and represents a preferred embodiment of the invention. The system, generally shown at 100 , is similar to the system 10 and will be understood from Figure 1 and the accompanying text. However, the system 100 differs in its configuration and selection of valve and pressure regulation components as will be described below.

The system 100 comprises an apparatus 111 coupled to a fluid conduit 132 via a suitable interface (not shown) and an isolation valve (not shown). A control system 150 in the form of a programmable logic controller (PLC) communicates with the apparatus 111 to set the parameters of operation and to control actuation of the valves of the apparatus. An external control panel (not shown) provides a user interface for the control system 150, and has controls for operating the frequencies of the valve oscillations, the maximum pressure differential in the system, as well as the maximum pressure and the minimum pressure in the system. The control panel also have an on/off switch, a pressure regulator override function, and visual indicators for the status of the various components of the system 100.

A fluid inlet 102 is connected to a fluid source (such as a tank) via a high pressure pump (not shown) and delivers fluid into the apparatus 111 via a particulate filter 104. An inlet pressure regulator 106 controls the pressure fluid delivered to the accumulator 108 via check valve 107, with excess fluid (over a predetermined pressure) diverted to a return line 110 via conduit 112. Therefore the inlet pressure regulator 106 delivers fluid to the accumulator 108 at a predetermined rate, set via the control system 150 .

34 The pressure accumulator 108 prevents loss of amplitude during the transmission of pulses, as is described in relation to the embodiment of Figure 1. Pressure within the 
accumulator is controlled by a pressure relief valve 114 disposed between the accumulator 108 and the return line 110 . The pressure relief valve is an oil hydraulically operated proportional pressure relief valve, designed to be capable of operating at a pressure of 500 bar (50 MPa), and a flow area diameter of up to 40 millimetres. An example of a suitable valve is the DN40 PN500 pressure relief valve available from $\mathrm{HL}$ Hydraulik $\mathrm{GmbH}$.

The apparatus 111 is also provided with an emergency pressure relief line 116 which bypasses the pressure relief valve 114 and includes an emergency stop actuation which bleeds all pressure in the accumulator to the return line 110.

The apparatus 111 comprises a first oscillating valve 120 which is hydraulically actuated from the control system 150 . The oscillating valve 130 is a pilot operated check valve designed to be capable of operating at a pressure of $500 \mathrm{bar}(50 \mathrm{MPa})$ and a flow rate of 500 litres per minute. An example of a suitable valve is the pilot operated check valve DN40 PN500 available from HL Hydraulik $\mathrm{GmbH}$. Actuation of the valve 130 allows a controlled pulse or series of pulses to be input into fluid conduit 132 in a similar manner to the system 10 of Figure 1.

The apparatus also includes a second oscillating valve 140 which is actuated by the control system 150. The valve 140 is a two-way hydraulic directional valve which can be piloted to open or close from an external oil hydraulic line. An example of a suitable valve is the two-way hydraulic directional valve DN40 PN500 available from HL Hydraulik $\mathrm{GmbH}$. In the pressure up cycle, the valve 140 is preferably in an open position, but it functions to operate cyclically in a pressure bleed cycle of the apparatus (analogous to the valves 26 and 36 of the system 10). The valve 140 is disposed between the fluid conduit 132 and the return line 110 , to allow return flow of fluid to the line 110 via a controllable pressure relief valve 142 .

Pressure sensor 123 measures the pressure $\mathrm{P} 1$ in the apparatus between the accumulator 106 and the valve 130 and provides a signal to the control system 150. Similarly, pressure sensor 129 measures the pressure $\mathrm{P} 2$ in the line between the valve 130 and the fluid conduit 132 (i.e. the fluid conduit pressure), and pressure sensor 137 measures the pressure $\mathrm{P} 3$ between the valve 140 and the pressure relief valve 142, both providing signals to the control system 150 . 
The control system 150 actuates the valves $130,140,114,142$ via oil filled hydraulic lines 113 (only some of which are shown for clarity). In this embodiment, the pilot medium in the lines 113 has an operating pressure sufficiently high to allow rapid actuation of the valves. In particular, preferred embodiments of the invention are configured to operate the oscillating valves 130,140 at pulse frequencies of greater than $1 \mathrm{~Hz}$. To facilitate this, the pilot medium pressure in lines 113 is greater than $20 \mathrm{MPa}$ (and typically around $30 \mathrm{MPa}$ ) in this embodiment of the invention. With the valve components selected, pulse frequencies of 1 to $10 \mathrm{~Hz}$ are contemplated by the invention.

Operation of the system 100 is similar to operation of the system 10 . In an initial configuration the valve oscillator 130 will normally be closed, and valve 140 will be in its open position. The operator enters the settings in the control system 150 , which include the operating frequencies of the valve oscillators 130 and 140 , the maximum differential pressure $(\mathrm{dP})$, the maximum pressure and the minimum pressure. It should be noted that the maximum pressure in the line can be controlled by the pressure relief valve 142, which

16 is exposed to fluid conduit 132. To begin unblocking the conduit 132, the pump (not shown) is activated to pump fluid from a fluid tank through the inlet regulator 106 and the check valve 107 of the accumulator 108. The oscillator valve 130 remains closed, and pressure sensor P2 takes a pressure measurement in the conduit 132). The control system 150 reads the pressure signal and adjusts the pressure relief valve 114 to control the pressure at $\mathrm{P} 1$ to a value within a pre-determined range (for example plus or minus $5 \%$ ) of a preset value of $\mathrm{P} 2+\mathrm{dP}$. When the desired value of $\mathrm{P} 1$ is reached, the control system 150 commands the oscillator valve 130 to cyclically open and close at its preset frequency (for example $3 \mathrm{~Hz}$ ). Positive pressure pulses are therefore transmitted into the conduit 132 to begin to remove the blockage. Transmission of pressure pulses increases the pressure $\mathrm{P} 2$, and therefore during the transmission of pulses, the valve 114 is automatically adjusted by the control system 150 to maintain the pressure $\mathrm{P} 1$ within the required range of $\mathrm{P} 2+\mathrm{dP}$.

When the pressure P2 in the fluid conduit reaches a preset maximum, the bleed-down cycle commences. Valve 130 is closed and optionally pressure is bled from the accumulator to return line 110 . Pressure at $P 3$ is initially equalised to the pressure $P 2$ in the fluid conduit, before the valve 140 is closed. The pressure relief valve 142 bleeds pressure from P3 until the differential pressure across valve 140 (i.e. P2 - P3) is at the 
frequency (for example $3 \mathrm{~Hz}$ ), which generates negative pressure pulses in the fluid conduit 132 as pressure is bled from the conduit 132. This has the effect of increasing the pressure $\mathrm{P} 3$ and decreasing the pressure $\mathrm{P} 2$. During the transmission of pulses, the pressure relief valve 142 is automatically adjusted by the control system 150 to maintain the pressure $\mathrm{P} 3$ within the required range of $\mathrm{P} 2-\mathrm{dP}$. When the minimum pressure is

6 reached in the fluid conduit 132, the process can be repeated.

The use of proportional pressure relief valves to control the pressure regulation advantageously allows a mode of operation in which the pressure differential is regulated during a pulse series. For example, the increase in pressure P3 during a pressure down cycle may be balanced by the proportional pressure relief valve, which is open sufficiently to bleed pressure to maintain the pressure differential within a desired range. Alternatively, the pressure relief valve can be operated after one pulses or a series of pulses to reset the pressure differential before the next pulse or pulses are generated.

The system 100 provides similar advantages as the system 10, principally by allowing the generation of pressure pulses of known amplitude throughout the pressure-up and bleeddown cycles. Providing amplitude control allows the parameters of the system to be set closer to the acceptable limits of the fluid conduit, with a higher level of confidence that the conduit 132 will not be damaged. The valve components and pressure regulation components of are particularly suited to conduits with inner diameters of around 2 to 12 inches (about 50 to $300 \mathrm{~mm}$ ) and find particular commercial application in conduits of 2 to 12 inches (about 100 to $250 \mathrm{~mm}$ ). The use of hydraulically-actuated valves with pilot medium pressures of greater than $20 \mathrm{MPa}$ (and preferably around $30 \mathrm{Mpa}$ ) provides the advantages of quick actuation and automated operation. This facilitates operation at high frequencies without reliance on human operators to manually open and close the valves. The choice of valves has the additional benefit of producing well-defined, repeatable pulses which may be detected in or near the fluid conduit to locate the blockage using known transit time techniques.

The invention provides a method and apparatus for removing a blockage from a fluid conduit. An apparatus comprises a first portion containing a fluid volume separated from the fluid conduit via a controllable valve. The valve is cyclically opened and closed such that a pressure differential between the first portion and the fluid conduit causes a series of 
WO 2011/036502

PCT/GB2010/051623

1 pressure pulses in the fluid conduit. The pressure differential is regulated to control the

2 amplitude of the pressure pulses of the series.

3

4 Variations to the described embodiments may be made within the scope of the invention.

5 In particular, it will be appreciated that components of the systems 10 and 100 may be

6 interchanged with one another in alternative embodiments of the invention, and that

7 combinations of features other than those expressly claimed are within the scope of the 8 invention.

9

10 


\section{Claims}

1. A method for removing a blockage from a fluid conduit, the method comprising: providing an apparatus comprising a first portion containing a fluid volume separated from the fluid conduit via a controllable valve; cyclically opening and closing the controllable valve such that a pressure differential between the first portion and the fluid conduit causes a series of pressure pulses in the fluid conduit; regulating the pressure differential to control the amplitude of the pressure pulses of the series.

2. The method as claimed in claim 1 comprising regulating the pressure of the fluid volume in the first portion so that it is greater than the pressure in the fluid conduit; and transmitting positive pressure pulses to the fluid conduit.

3. The method as claimed in claim 1 or claim 2 comprising regulating the pressure of the fluid volume in the first portion so that it is less than the pressure in the fluid conduit; and transmitting negative pressure pulses to the conduit.

4. The method as claimed in claim 3 comprising transmitting a series of positive pressure pulses into the system during a pressuring up cycle and transmitting a series of negative pressure pulses during a pressure bleeding cycle.

5. The method as claimed in any preceding claim comprising maintaining the pressure differential within preferred predetermined range.

6. The method as claimed in any preceding claim comprising measuring fluid pressure in the fluid conduit.

7. The method as claimed in claim 6 comprising measuring an average pressure in the fluid conduit over a period of at least one pulse cycle.

8. The method as claimed in claim 6 or claim 7 comprising measuring a first fluid pressure in the first portion, and calculating the pressure differential from the first fluid pressure and the fluid pressure in the fluid conduit. 
9. The method as claimed in any of claims 6 to 8 wherein the first and/or second fluid pressure measurements are communicated to a control module.

4

10. The method as claimed in any preceding claim comprising controllably operating the valve by a control module.

11. The method as claimed in any preceding claim comprising directing fluid through a second controllable valve by cyclically opening and closing the second controllable valve.

12. The method as claimed in claim 11 wherein the second controllable valve is located on a fluid return line.

13. Apparatus for removing a blockage from a fluid conduit or vessel, the apparatus comprising: a first portion containing a fluid volume; a connector for coupling the first portion to the fluid conduit or vessel; a controllable valve disposed between the first portion and the connector; at least one pressure sensor for measuring a pressure in the fluid conduit or vessel; a control module for opening and closing the valve; and a fluid pressure regulator configured to control the fluid pressure in the first portion in response to a signal from the pressure sensor.

14. The apparatus as claimed in claim 13 configured to cyclically open and close the valve to transmit pressure pulses into a fluid conduit to remove a blockage.

15. The apparatus as claimed in claim 13 or claim 14 configured to measure a differential pressure, which may be a differential pressure across the valve.

16. The apparatus as claimed in any of claims 13 to 15 , wherein the pressure regulator comprises a pressure relief valve.

17. The apparatus as claimed in any of claims 13 to 16 , wherein the pressure regulator comprises a two-way pressure regulator. 
18. The apparatus as claimed in any of claims 13 to 17 , wherein the pressure regulator is electronically controllable.

19. The apparatus as claimed in any of claims 13 to 18 , comprising a control module for configuring operational parameters of the apparatus selected from the group consisting of: operating frequency; pulse width; maximum differential pressure $(\mathrm{dP})$; maximum pressure; and minimum pressure.

20. The apparatus as claimed in any of claims 13 to 19 , comprising a fluid return line from the fluid conduit.

21. The apparatus as claimed in claim 20 , comprising a second valve disposed between the fluid conduit and the fluid return line.

22. The apparatus as claimed in claim 21 , wherein the second valve is configured for controllable transmission of fluid pressure pulses.

23. The apparatus as claimed in claim 21 or claim 22 , comprising means for regulating a pressure differential across the second valve.

24. The apparatus as claimed in any of claims 13 to 23 , wherein at least one of the valve and/or the second valve is an oscillating valve.

25. The apparatus as claimed in claim 24 , wherein at least one of the valve and/or the second valve is hydraulically operable.

26. The apparatus as claimed in claim 25 , wherein the hydraulically operable valve is actuable by a hydraulic line at a pressure in excess of $20 \mathrm{Mpa}$.

27. The apparatus as claimed in any of claims 24 to 26 , wherein least one of the valve and/or the second valve is electronically operable.

28. A hydrocarbon production or transportation system comprising a fluid conduit and an apparatus for removing a blockage from the fluid conduit coupled to the conduit, the system comprising a first portion containing a first fluid volume; 
a controllable valve disposed between the first portion and the fluid conduit; a pressure source for providing pressurised fluid to the first portion; a control module configured for opening and closing the valve to allow pressure pulses into the fluid conduit;

5 pressure sensing means for determining a pressure differential across the 6 controllable valve; and a fluid pressure regulator configured to control the fluid pressure in the first portion in response to a signal from the pressure sensing means. 
WO 2011/036502

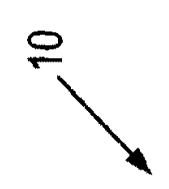

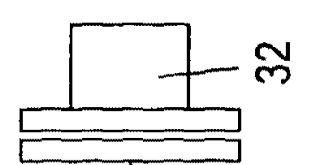

$\frac{1}{2}$

PCT/GB2010/051623

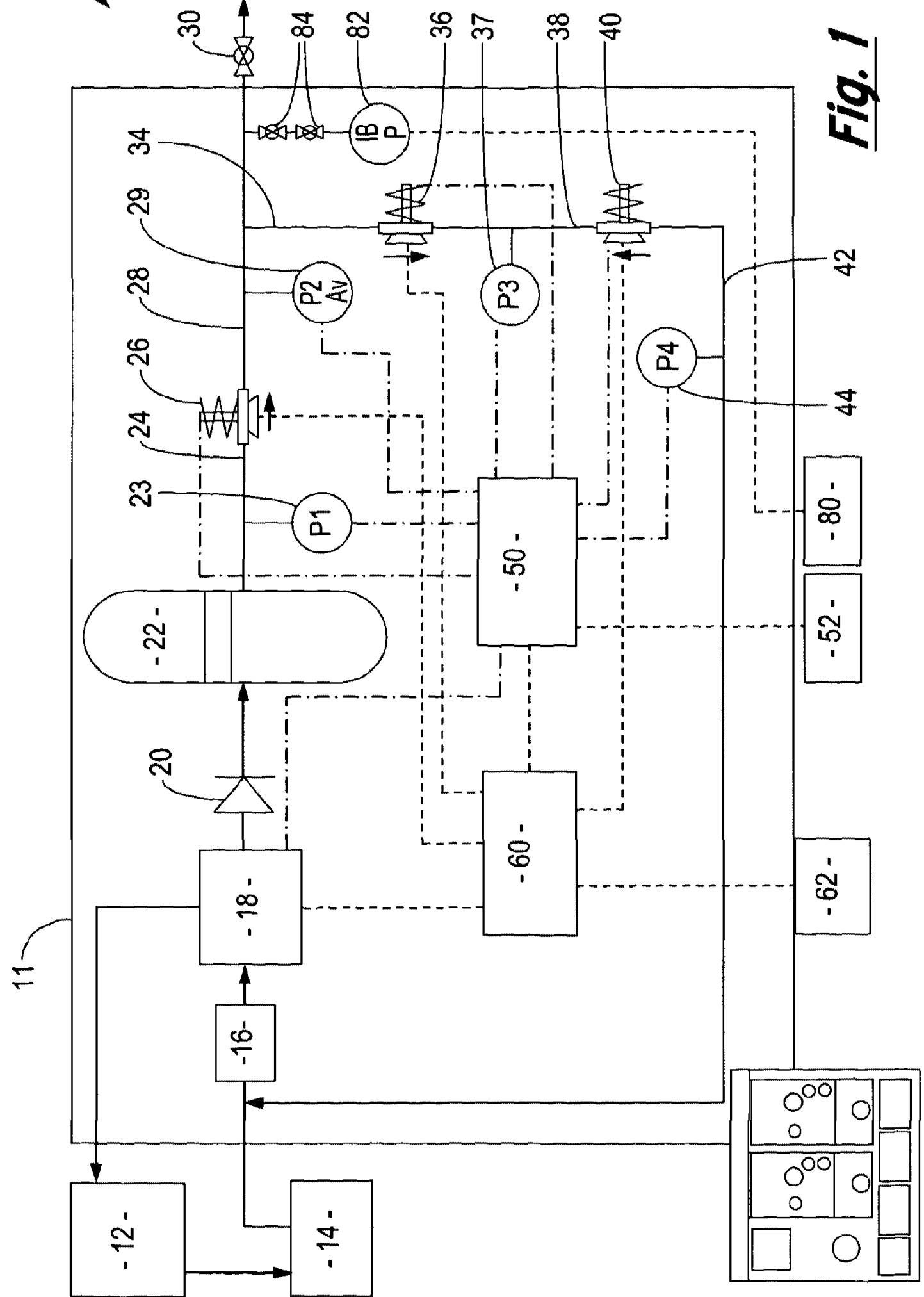

SUBSTITUTE SHEET (RULE 26) 


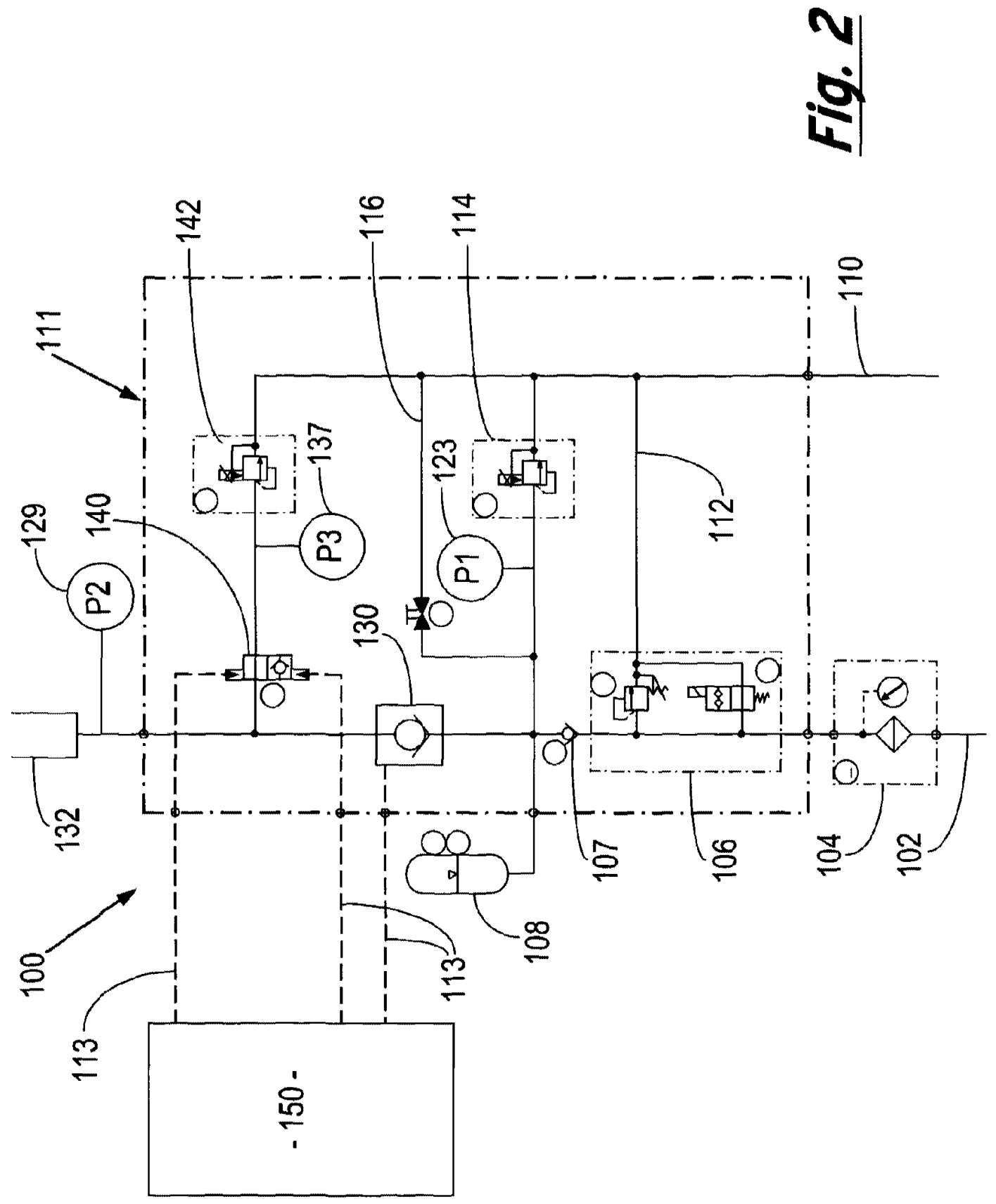


RPP-RPT-53783, Rev. 0

Appendix F

DILUENT AND FLUSH BUILDING COSTS 
From: Kraemer, Kyle E

Sent: Friday, October 19, 2012 2:51 PM

To: Garfield, John S

Cc: Whitcomb, Jeremy J

Subject: RE: Diluent and Flush Building Costs

John,

The following are the baseline values we have for the AY/AZ Diluent Flush Building (NOTE: All are fully burdened cost and include WRPS field labor support):

- Retaining Wall/Stairs/Slab (\$474K)

- Procure 17,500 gal tank (\$211K)

- Procure/Install Mechanical $(\$ 1,457 \mathrm{~K})$

- Procure/Install Electrical/Instrumentation (\$576K)

- Procure In-Line Heaters (\$446K)

- Fabricate/Install Building Structure $(\$ 201 \mathrm{~K})$ NOTE: The current design/estimate is only for a roof structure, not a full building.

- Mechanical Testing $(\$ 159 \mathrm{~K})$

- Electrical Testing $(\$ 62 \mathrm{~K})$

TOTAL $(\$ 3,586 \mathrm{~K})$

Let me know if you need anything else.

Thanks.

Kyle Kraemer

Project Controls

Tank Farm Projects

BNL Tech

Subcontractor to WRPS

Contractor to US DOE

373-4845

2752E/D109 
RPP-RPT-53783, Rev. 0

Appendix G

HYDROKINETICS CORRESPONDENCE 
From: Brooks Bradford, Jr. [mailto:Brooks@aimmtechnologies.com]

Sent: Friday, December 14, 2012 11:38 AM

To: John Garfield

Subject: RE: Follow up Questions about the Hydrokinetics System

Ok thanks.

From: John Garfield [mailto:jgarfield@aemconsultingllc.com]

Sent: Friday, December 14, 2012 1:37 PM

To: Brooks Bradford, Jr.

Subject: RE: Follow up Questions about the Hydrokinetics System

The document will be distributed internally within our own company, to the Richland office of DOE, and possible to Dwayne McDaneil at FIU.

As a publically released document anyone can request it through freedom of information.

John S. Garfield

AEM Consulting, LLC

1201 Jadwin Ave., Ste. 203

Richland, WA 99352

Office: 509.946 .3685

Cell: 509.308.6873

Email: igarfield@aemconsultingllc.com

From: Brooks Bradford, Jr. [mailto:Brooks@aimmtechnologies.com]

Sent: Friday, December 14, 2012 11:28 AM

To: John Garfield

Subject: RE: Follow up Questions about the Hydrokinetics System

Yes I approve.

Who is this document being sent to? 
From: John Garfield [mailto:jgarfield@aemconsultingllc.com]

Sent: Friday, December 14, 2012 1:27 PM

To: Brooks Bradford, Jr.

Subject: RE: Follow up Questions about the Hydrokinetics System

Hi Brooks,

Thanks for your response. Can I assume that our use of the picture and narrative description in the document has your approval from an NDA standpoint?

Please let me know.

Thanks,

John S. Garfield

AEM Consulting, LLC

1201 Jadwin Ave., Ste. 203

Richland, WA 99352

Office: 509.946 .3685

Cell: 509.308 .6873

Email: igarfield@aemconsultingllc.com

From: Brooks Bradford, Jr. [mailto:Brooks@aimmtechnologies.com]

Sent: Wednesday, December 12, 2012 9:34 AM

To: John Garfield

Subject: RE: Follow up Questions about the Hydrokinetics System

Sorry for the delay - year end is always fun.

Just reviewed the document - everything looks good.

Brooks Bradford, Jr.

From: John Garfield [mailto:jgarfield@aemconsultingllc.com]

Sent: Wednesday, December 12, 2012 11:29 AM

To: Brooks Bradford, Jr.

Subject: RE: Follow up Questions about the Hydrokinetics System

Hi Brooks,

We trying to issue our document for public release and I need your agreement that there is no violation of our NDA regarding the pictures Anton sent us and the narrative of the operating approach attached.

An email granting that permission is probably sufficient.

Thanks 509-308-6873

John S. Garfield

AEM Consulting, LLC

1201 Jadwin Ave., Ste. 203

Richland, WA 99352

Office: 509.946 .3685

Cell: 509.308.6873

Email: igarfield@aemconsultingllc.com 
From: Brooks Bradford, Jr. [mailto:Brooks@aimmtechnologies.com]

Sent: Friday, November 16, 2012 9:18 AM

To: John Garfield

Subject: RE: Follow up Questions about the Hydrokinetics System

John -

Here are the answers top your questions.

Thanks,

Brooks

1. Can we receive a process flow diagram, and equipment list for the hydrokinetics system?

a. Attached

2. As mentioned before there are expansion joints every $600 \mathrm{ft}$ (see attached illustrations) within the cross site transfer pipelines in our application, and these lines can reach $29000 \mathrm{ft}$ long. Would the pressures pulsation be attenuated by expansion joints? Would the expansion joint cause undesirable line vibration and/or harmonic oscillation that would damage the pipeline? (Similar to a water hammer phenomena) How do you manage these issues?

a. I did not see the illustrations, but we have never had any issues with expansion joints, nor have we seen any pressure spikes at these locations.

3. What is the standard offering and cost? Need a basis to understand the modifications for our application. Also, what is the cost to purchase the system since the system may be radioactively contaminated?

- Typically we charge by the shift - between $\$ 8,000$ and $\$ 20,000$ depending on the application. We have only sold our equipment once in the history of the company and that was an exception for a client we had a very long relationship with. We sold the entire package (Hydrokinetic equipment, hydroblast pump, hoses, accessories, etc) for $\$ 512,000$.

4. How is the air removed from the line to get the pressure pulse in contact with the plug after the line is drained?

a. This is on a case by case basis. The best method for us is when the line is full of liquid. If it has been previously drained, we can place a vacuum on the line, or strategically full the line to reduce the amount of air in the line. 
5. What is a typical length of operation to successfully remove a blockage? For example a blockage caused by $A$ ) a viscous material such as a clay paste, $B$ ) a hardened material such as cement, $C$ ) settled denser particles, or D) crystallized salts.

a. Length that we can clean is determined by a few factors (overall length of the line, diameter, material type, and length of the plug). The easiest material for us to remove is any type of plastic polymer / compound; while the hardest is something like fully packed sand. Between those we typically can get communication $90 \%+$ of the time. On more dense materials, we must increase our pumping power, and in long blockage applications we will need to make sure we have volume and pressure to move the material - all while keeping within the pressure range of the pipe.

6. With the pressure limitations in our application, can the system reach above the cavitation threshold at either 275 psi or 400 psi? How about reaching above cavitation at approximately 1500 psi: the pipe rating for the longest route is schedule 40 stainless steel at 200F?

a. What is the OD / ID of the line? 400 PSI is at the bottom end of the pressures we work with - 1500 PSI would be ideal.

7. How do you determine the cavitation threshold? Do you monitor the acoustics (Pulse echo), monitor the line vibration, or by calculation?

a. Typically we just used the pressure readings. We have in the past attached different type of recording devices to the process for monitoring, but it is a rare occurrence for us. We can discuss what you would like to see.

8. How do you base the pulse amplitude and the overall Hydrokinetics operating pressure for both fluid and pulse pressure ( $P_{\text {fluid }}+D P_{\text {pulse }}$ ). Would an increase in pulse amplitude within the limits of the operating pressure (275-400) help removing the blockage?

a. An increase is amplitude would help at lower pressures, but we have never experimented with this to make it effective on a routine basis.

9. It seems in testing at FIU, the frequency of pulsation was manually determined by an operator pushing on the switches on the control panel. Can the frequency of pulsation be automated?

a. Yes - we have had an automated unit, but was much slower so we set that unit aside.

What is the desired frequency range?

- I will need to look into that. 
It was stated in a report by Oak Ridge National Lab (attached the PDF file) that pulse frequencies as high as 11,250 vibrations per minute $(\sim 190 \mathrm{~Hz})$ could be achieved. Were these high frequencies reached using automated actuating valves?

- This was before my time when the inventor of the process (Ralph Garcia) was doing R and D work. Let me look up that report on how that was achieved.

What are your thoughts about using pressure pulsation at both ends of plugged line to create asynchronous pulsing? The FIU has done some recent work in pulsing from both ends of a plugged pipe where Pressure pulses are created asynchronously in order to break the mechanical bonds between the blockage and the pipe walls as a result of the two-way wave action.

We have never attempted this, so I cannot speak to how well it would work. Typically we want the opposite end open, so the blockage and release down the line.

As I have stated before, our core business is in petrochemical and refining application. These applications do not require the scientific research and backing; therefore we have not performed / documented controlled experiments and testing. The main bulk of our testing happened about 10 years ago and was managed by Ralph Garcia, who has since passed away.

Please let me know if you have any additional questions.

Brooks Bradford, Jr.

From: John Garfield [mailto:jgarfield@aemconsultingllc.com]

Sent: Thursday, November 08, 2012 12:43 PM

To: Brooks Bradford, Jr.; Antone Belcher

Cc: 'Gita Golcar'; Gary Dunford

Subject: RE: Follow up Questions about the Hydrokinetics System

Hi Brooks,

Attached is the signed non-disclosure agreement. We would like you to review the document for any sensitivities at the draft and final stages.

I've included the original list of questions below... some of which Antone has already addressed. We are primarily interested in the additional pictures for the report, flow diagram or P\&ID for your standard setup (pumps, valves, tanks, control system, etc.), equipment list, specs on some of the key equipment items and an estimate to procure the equipment, cover the cost of a typical unplugging event by your staff, and perhaps a number for training our staff to use the equipment.

A phone conversation would be a great starting point. Please suggest a time that is good on your end.

Thanks,

1. Can we receive a process flow diagram, and equipment list for the hydrokinetics system? 
2. As mentioned before there are expansion joints every $600 \mathrm{ft}$ (see attached illustrations) within the cross site transfer pipelines in our application, and these lines can reach $29000 \mathrm{ft}$ long. Would the pressures pulsation be attenuated by expansion joints? Would the expansion joint cause undesirable line vibration and/or harmonic oscillation that would damage the pipeline? (Similar to a water hammer phenomena) How do you manage these issues?

3. What is the standard offering and cost? Need a basis to understand the modifications for our application. Also, what is the cost to purchase the system since the system may be radioactively contaminated?

4. How is the air removed from the line to get the pressure pulse in contact with the plug after the line is drained?

5. What is a typical length of operation to successfully remove a blockage? For example a blockage caused by $A$ ) a viscous material such as a clay paste, B) a hardened material such as cement, $C$ ) settled denser particles, or D) crystallized salts.

6. With the pressure limitations in our application, can the system reach above the cavitation threshold at either 275 psi or 400 psi? How about reaching above cavitation at approximately 1500 psi: the pipe rating for the longest route is schedule 40 stainless steel at 200F?

7. How do you determine the cavitation threshold? Do you monitor the acoustics (Pulse echo), monitor the line vibration, or by calculation?

8. How do you base the pulse amplitude and the overall Hydrokinetics operating pressure for both fluid and pulse pressure ( $P_{\text {fluid }}+D P_{\text {pulse }}$ ). Would an increase in pulse amplitude within the limits of the operating pressure (275-400) help removing the blockage?

9. It seems in testing at FIU, the frequency of pulsation was manually determined by an operator pushing on the switches on the control panel. Can the frequency of pulsation be automated? What is the desired frequency range? It was stated in a report by Oak Ridge National Lab (attached the PDF file) that pulse frequencies as high as 11,250 vibrations per minute ( $190 \mathrm{~Hz})$ could be achieved. Were these high frequencies reached using automated actuating valves? 
What are your thoughts about using pressure pulsation at both ends of plugged line to create asynchronous pulsing? The FIU has done some recent work in pulsing from both ends of a plugged pipe where Pressure pulses are created asynchronously in order to break the mechanical bonds between the blockage and the pipe walls as a result of the two-way wave action.

John S. Garfield

AEM Consulting, LLC

1201 Jadwin Ave., Ste. 203

Richland, WA 99352

Office: 509.946 .3685

Cell: 509.308 .6873

Email: igarfield@aemconsultingllc.com

From: Antone Belcher [mailto:belcher@aimmtechnologies.com]

Sent: Thursday, November 01, 2012 9:10 AM

To: Gita Golcar; 'John Garfield'

Cc: Brooks Bradford, Jr.

Subject: RE: Follow up Questions about the Hydrokinetics System

Gita and John,

Please read the attached Non-Disclosure/Non-Compete Agreement - if in agreement, please print - sign and return back to AIMM.

I will be available to discuss our cleaning method today on or around $4 \mathrm{pm}$ (CST) $-2 \mathrm{pm}$ your time.

Good Day,

Antone Belcher

AIMM Technologies, Inc.

409-945-5414 Phone

409-945-6022 Fax

www.aimmtechnologies.com

$\rightarrow \frac{\text { Ally }}{\text { Thingunin }}$ 


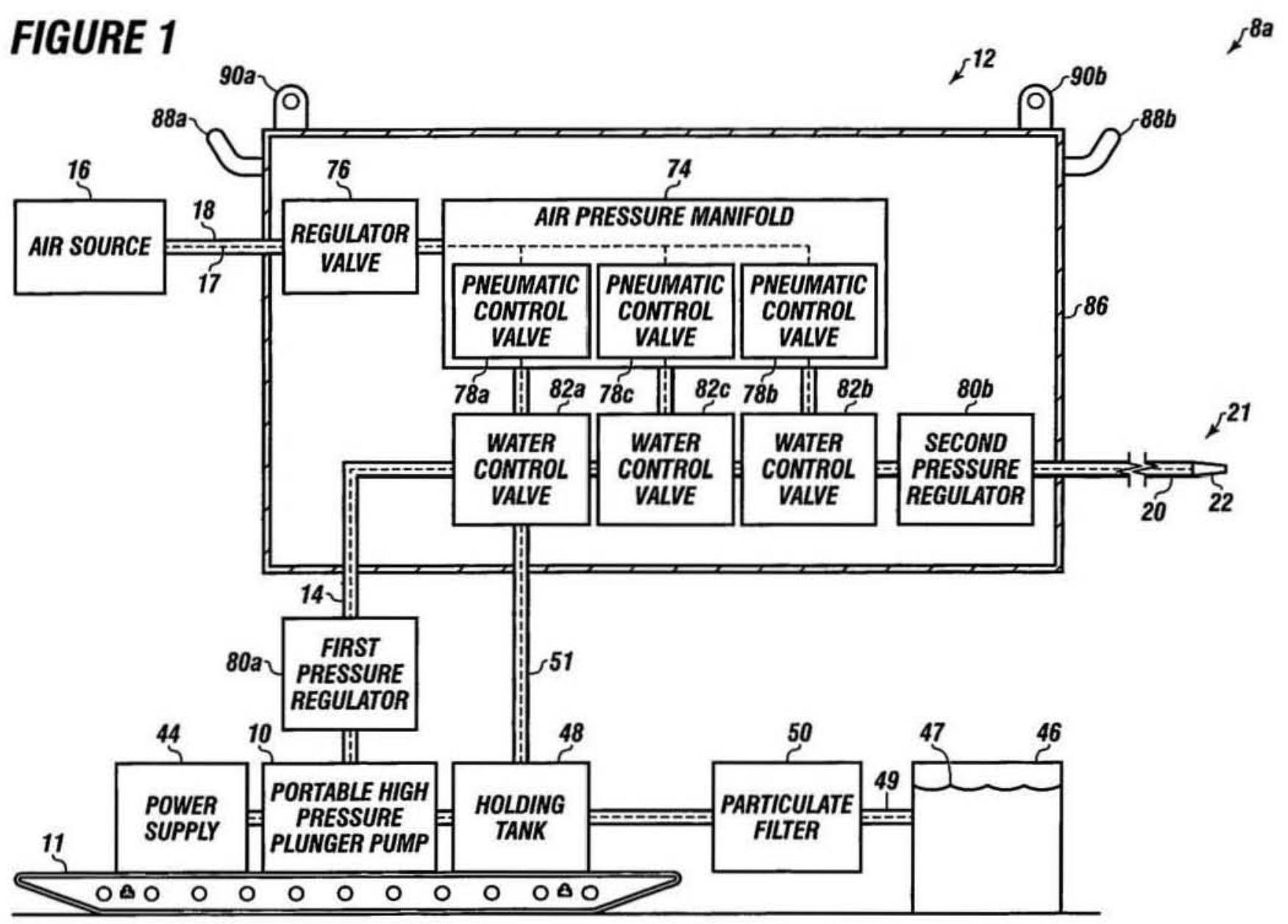

